\title{
Physical Property Modeling of Solvent-Based Carbon Capture Processes with Uncertainty Quantification and Validation with Pilot Plant Data
}

Joshua Cole Morgan

Follow this and additional works at: https://researchrepository.wvu.edu/etd

\author{
Recommended Citation \\ Morgan, Joshua Cole, "Physical Property Modeling of Solvent-Based Carbon Capture Processes with \\ Uncertainty Quantification and Validation with Pilot Plant Data" (2017). Graduate Theses, Dissertations, \\ and Problem Reports. 6262. \\ https://researchrepository.wvu.edu/etd/6262
}

This Dissertation is protected by copyright and/or related rights. It has been brought to you by the The Research Repository @ WVU with permission from the rights-holder(s). You are free to use this Dissertation in any way that is permitted by the copyright and related rights legislation that applies to your use. For other uses you must obtain permission from the rights-holder(s) directly, unless additional rights are indicated by a Creative Commons license in the record and/ or on the work itself. This Dissertation has been accepted for inclusion in WVU Graduate Theses, Dissertations, and Problem Reports collection by an authorized administrator of The Research Repository @ WVU.

For more information, please contact researchrepository@mail.wvu.edu. 


\title{
Physical Property Modeling of Solvent-Based Carbon Capture Processes with Uncertainty
} Quantification and Validation with Pilot Plant Data

\author{
Joshua Cole Morgan \\ Dissertation submitted to the \\ Statler College of Engineering and Mineral Resources \\ at West Virginia University \\ in partial fulfillment of the requirements for the degree of \\ Doctor of Philosophy in \\ Chemical Engineering \\ Debangsu Bhattacharyya, Ph.D., Chair \\ Brian Anderson, Ph.D. \\ David Mebane, Ph.D. \\ David C. Miller, Ph.D. \\ Richard Turton, Ph.D. \\ Stephen Zitney, Ph.D. \\ Department of Chemical and Biomedical Engineering \\ Morgantown, West Virginia \\ 2017
}

Keywords: Carbon capture, thermodynamics, monoethanolamine, uncertainty quantification, design of experiments

Copyright 2017 Joshua C. Morgan 


\section{ABSTRACT \\ Physical Property Modeling of Solvent-Based Carbon Capture Processes with Uncertainty Quantification and Validation with Pilot Plant Data Joshua C. Morgan}

The US DOE's Carbon Capture Simulation Initiative (CCSI) has a strong focus on the development of state of the art process models to accelerate the development and commercialization of post-combustion carbon capture system technologies. One of CCSI's goals is the development of a rigorous process model that may serve as a definitive reference for benchmarking the performance of solvent-based $\mathrm{CO}_{2}$ capture systems, using aqueous monoethanolamine (MEA) as a baseline. Among the requirements of this process model is the development of its component submodels (e.g. physical properties) from relevant bench-scale data. Moreover, the process model must take into account parametric uncertainty in the submodels and be validated with both steady-state and dynamic process data collected from a pilot plant over a wide range of operating conditions. This dissertation is focused on two major aspects of the development of this MEA solvent model, namely the development of the physical property models for the MEA- $\mathrm{H}_{2} \mathrm{O}-\mathrm{CO}_{2}$ system and the validation of the steady-state model with large-scale pilot plant data from the National Carbon Capture Center (NCCC) in Alabama.

The physical property modeling work may be divided into standalone property models and the integrated thermodynamic framework of the system. Viscosity, density, and surface tension models have been developed individually by calibrating parameters, for an empirical model of a given form, to fit experimental data from the open literature. The thermodynamic framework has been developed within Aspen Plus ${ }^{\circledR}$, using the e-NRTL model as a starting point, by regressing model parameters to fit vapor-liquid equilibrium (VLE), heat capacity, and heat of absorption data. A parameter selection methodology using an information criterion has been implemented for reducing the model complexity. A methodology for uncertainty quantification (UQ) has also been included for all property models, in which Bayesian inference is used to update distributions of model parameters in light of experimental data.

The physical property models, along with separately developed mass transfer, hydraulic, and reaction kinetics models, are incorporated into the overall process model. This model has been validated with steady-state data from NCCC for a total of 23 test runs, and the model predictions of absorber and stripper column performance have been shown to match the experimental data with satisfactory fit. The parametric uncertainty from the process submodels are propagated through the process model in order to study the resulting uncertainty in the process variables of the system, notably the $\mathrm{CO}_{2}$ capture percentage of the absorber and the amount of $\mathrm{CO}_{2}$ regenerated in the stripper. Concurrent sensitivity studies have been performed, which provide insight into the relative contributions of the uncertainty in particular submodels to the overall process uncertainty.

Finally, some ongoing work related to the solvent model project is also presented. In one project, a methodology for scale-up uncertainty quantification is being developed, in which the effect of radial liquid distribution on column performance is estimated and preliminary comparison of this 
model to process data is made. The final project involves using the completed process model for planning a second MEA campaign at NCCC, which is ongoing at the time of the writing of this dissertation. In this work, the estimated uncertainty in absorber efficiency is quantified as a function of key manipulated variables by propagating the submodel parametric uncertainty through the absorber model over the range of input variables. An initial set of test conditions has been designed with the objective of choosing points for which the estimated uncertainty is relatively high, while maintaining a spread of the conditions throughout the input space. A methodology has been proposed for using Bayesian inference to update the parametric uncertainty as the data are collected. 
Dedication

To my family 


\section{Acknowledgements}

My pursuit of a Ph.D. in chemical engineering at West Virginia University has been a highly satisfying experience, and I must give thanks to all those who contributed to my success. I would like to thank my advisor, Dr. Debangsu Bhattacharyya, for being a very demanding, yet patient, mentor who has always pushed me to provide high quality research work, which has allowed me to become a better engineering researcher in the process. I would also like to thank the members of my Advisory and Examining Committee (Dr. Brian Anderson, Dr. David Mebane, Dr. David Miller, Dr. Richard Turton, and Dr. Stephen Zitney) for their guidance on this research work, particularly during the research proposal phase of this project. I have been honored to work among a great group of students (Anderson, Qiao, Yuan, Pratik, Prokash, Dustin, Qiang, Jacob, Sarah, Samson, Chirag, Anca, Yifan, Paul, Ryan, Goutham, Pushpitha, Christian, Katie, Elijah) in Prof. Bhattacharyya's Advanced Process and Energy Systems Engineering Group, both past and current. I have enjoyed the opportunity to get to know many of you, and to have the opportunity to discuss research work and share ideas with many of you. I maintain fond memories of all of the adventures we have had outside of the work setting, particularly on travels for AIChE conferences. I would especially like to acknowledge the honor of working directly with Anderson Soares Chinen on the development of the MEA solvent model and various other projects; without his contributions, most of the work contained herein would not be possible. Similarly, I would like to thank Ben Omell for his very valuable guidance throughout the development of these process models, and for providing the results for the validation of our model with TCM data.

There are many collaborators that I have been privileged to work with, and who have contributed much to the work described in this document. I would like to thank the National Carbon Capture Center (NCCC) and all of the involved staff for providing data for the 2014 and 2017 test campaigns for the MEA solvent system. I would like to thank Charles Tong from Lawrence Livermore National Laboratory for providing much assistance and advice for the uncertainty quantification phase of this work, particularly with respect to use of the PSUADE software, and for his patience while I learned to use these tools. I have also enjoyed the opportunity to collaborate with Sham Bhat and Christine Anderson-Cook from Los Alamos National 
Laboratory on the design of experiments for the 2017 NCCC test campaign, and acknowledge their contributions to the success of this project. I would like to thank Bill Buschle from the University of Edinburgh for his work on the measurement uncertainty in the solvent at NCCC, which has yielded results that have greatly improved the comparison between our MEA model and the pilot plant data. I would like to thank Prof. Gary Rochelle from the University of Texas at Austin for sharing the "Phoenix Model", which was used as a precursor to the model developed in this work, and members of his research team, specifically Brent Sherman, for being very helpful as I was starting out in the solvent modeling work. I would like to thank all involved in the high viscosity solvent model project for the opportunity to be involved with this collaborative effort. I am grateful for the CCSI and $\mathrm{CCSI}^{2}$ research programs, and for the leadership of David Miller and Michael Matuszewski, that have made these collaborations possible.

While pursuing a Ph.D. at West Virginia University, I have been privileged to be able to supplement my research with excellent coursework from within the Department of Chemical and Biomedical Engineering (CBE) and elsewhere. From CBE, I would like to acknowledge the following professors for offering excellent courses that have contributed to my research and professional development in some way: Prof. Charter Stinespring (thermodynamics and statistical and molecular thermodynamics), Prof. Ray Yang (transport phenomena and reaction engineering), Prof. David Klinke (mathematical methods in chemical engineering), Prof. Rakesh Gupta (mass transfer), Prof. John Zondlo (heat transfer), Prof. Debangsu Bhattacharyya (optimization). I would also like to acknowledge courses from Prof. Gary Ganser (mathematical modeling) and Prof. Erdogan Gunel (statistical theory) from outside the CBE department.

I am grateful to a great group of friends (Scott, Brendan, Nick, Zach, Michael, et al.) who have always been willing to take time out of their busy lives to return to Morgantown to visit me and watch football. I would like to thank my family for always encouraging me to pursue lofty goals and holding me to very high standards throughout my life.

Finally, I would like to acknowledge financial support for this research. This research was conducted through the Carbon Capture Simulation Initiative (CCSI), funded through the U.S. 
DOE Office of Fossil Energy. A portion of this work was funded by Lawrence Berkeley Laboratory through contract\# 7210843. 


\section{Disclaimer}

This paper was prepared as an account of work sponsored by an agency of the United States Government. Neither the United States Government nor any agency thereof, nor any of their employees, makes any warranty, express or implied, or assumes any legal liability or responsibility for the accuracy, completeness, or usefulness of any information, apparatus, product, or process disclosed, or represents that its use would not infringe privately owned rights. Reference herein to any specific commercial product, process, or service by trade name, trademark, manufacturer, or otherwise does not necessarily constitute or imply its endorsement, recommendation, or favoring by the United States Government or any agency thereof. The views and opinions of authors expressed herein do not necessarily state or reflect those of the United States Government or any agency thereof. 


\section{Table of Contents}

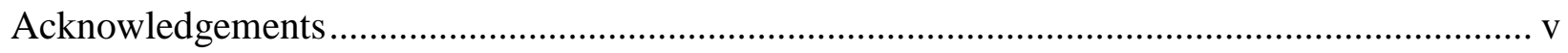

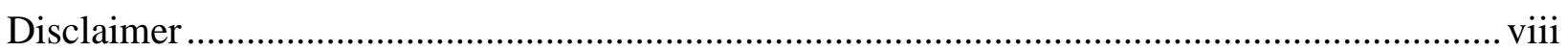

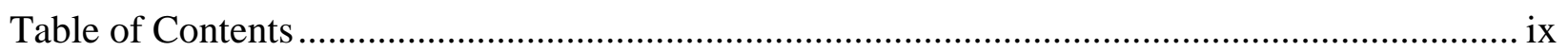

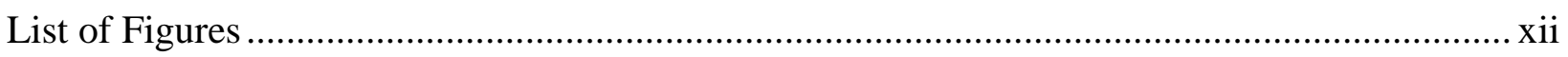

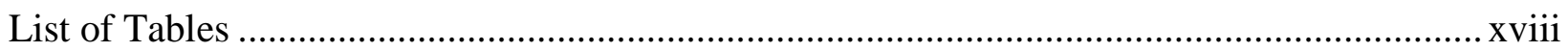

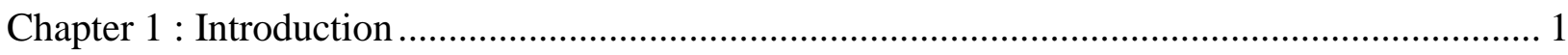

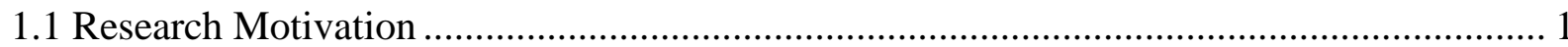

1.2 Physical Property Modeling with Uncertainty Quantification......................................... 2

1.3 Carbon Capture with Aqueous MEA System ................................................................. 4

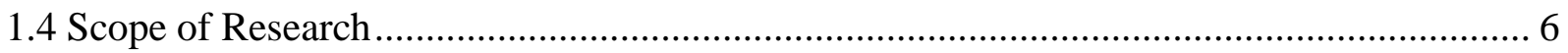

Chapter 2 : Property Modeling Overview .............................................................................. 9

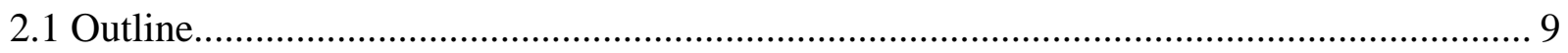

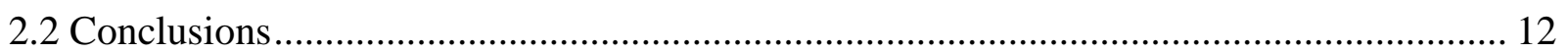

Chapter 3 : MEA Physical Property Models (Deterministic) ................................................. 13

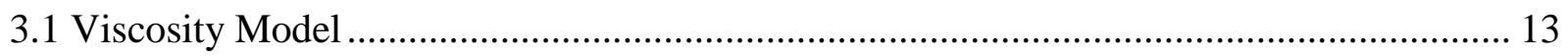

3.2 Molar Volume Model ................................................................................................. 15

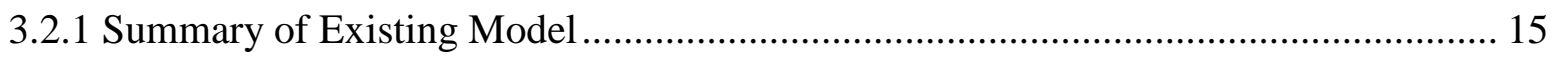

3.2.2 Modified Model ........................................................................................... 16

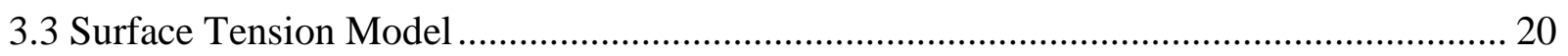

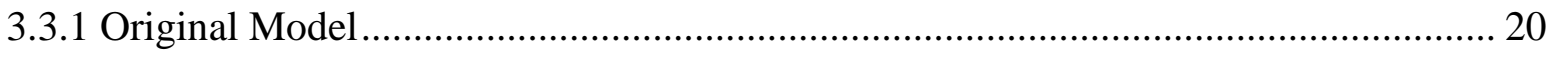

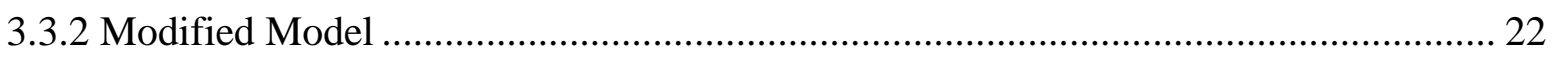

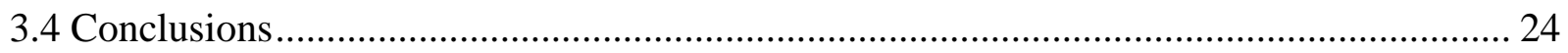

Chapter 4 : MEA Thermodynamic Framework ..................................................................... 26

4.1 Background for ELECNRTL Method in Aspen Plus ................................................... 26

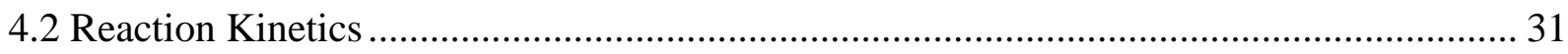

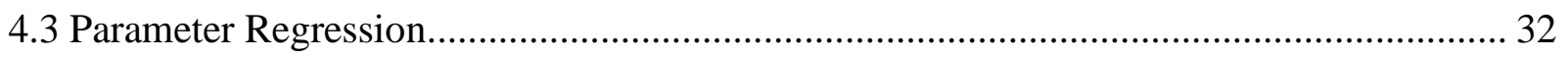

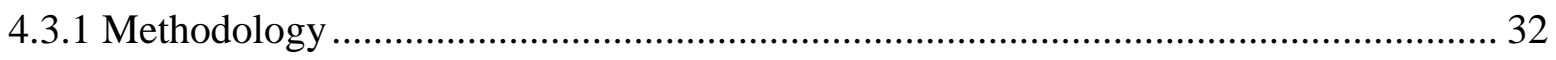

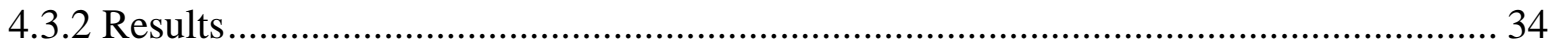

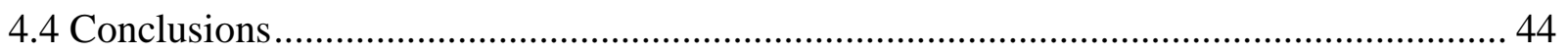

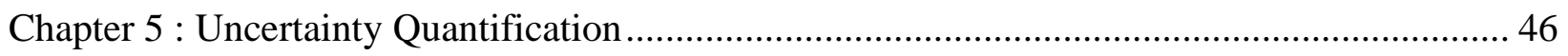

5.1 Standalone Physical Property Models ........................................................................ 46 
5.1.1 Parameter Selection and Prior Selection............................................................... 46

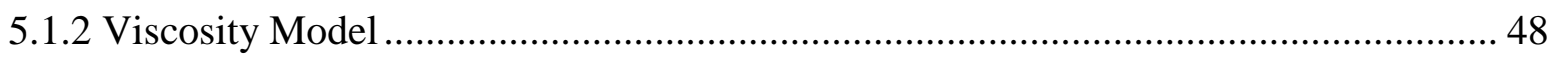

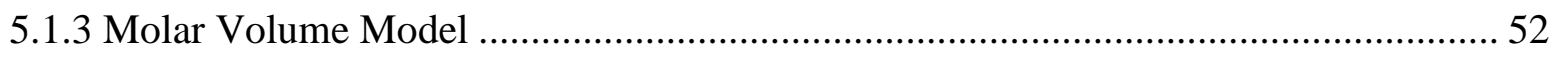

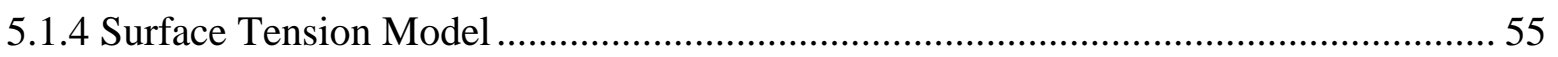

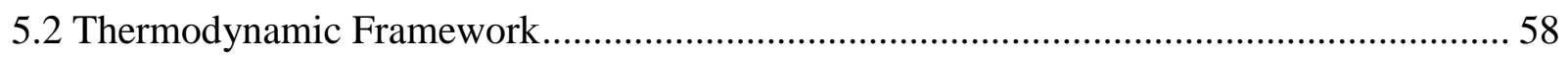

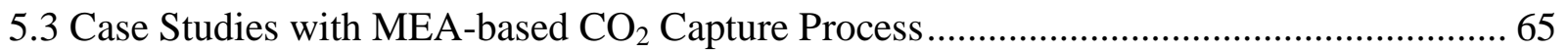

5.3.1 Case Study with Viscosity, Molar Volume, and Surface Tension Models................... 65

5.3.2 Case Study with Thermodynamic Framework ............................................................ 72

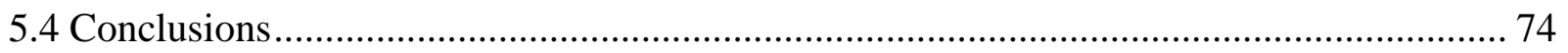

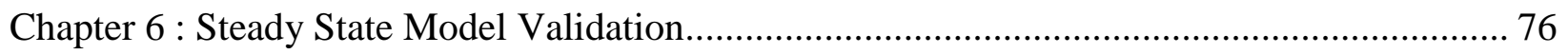

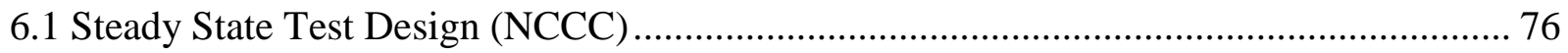

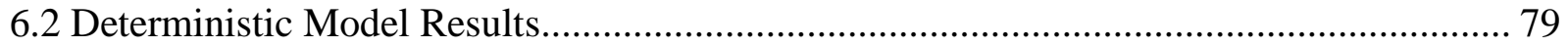

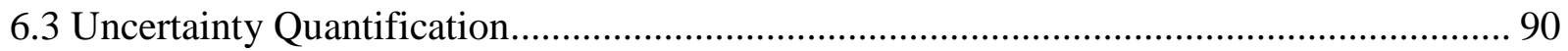

6.3.1 Physical Property Model Parameters …………......................................................... 90

6.3.2 Incorporation of Mass Transfer and Hydraulic Model Parameters .............................. 95

6.4 Measurement Uncertainty Analysis ............................................................................. 102

6.5 TCM Test Campaign............................................................................................. 108

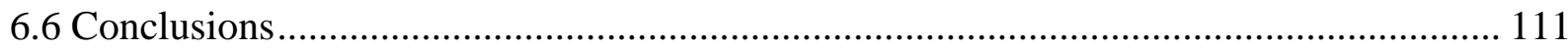

Chapter 7 : NCCC MEA Test Campaign (Summer 2017) Planning ........................................ 113

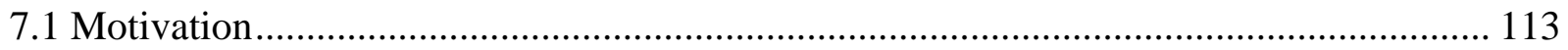

7.2 Deterministic Model Performance ........................................................................... 114

7.2.1 Absorber Column (3 Beds with Intercooling) ....................................................... 114

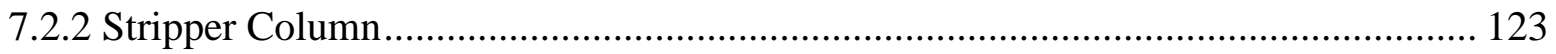

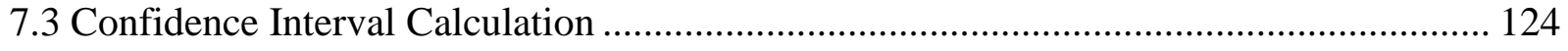

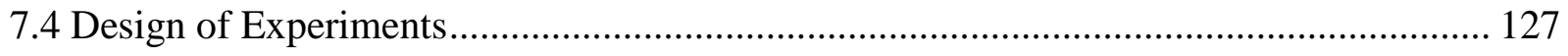

7.5 Alternative Process Configurations ........................................................................... 138

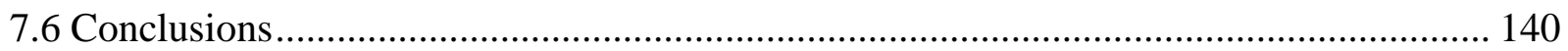

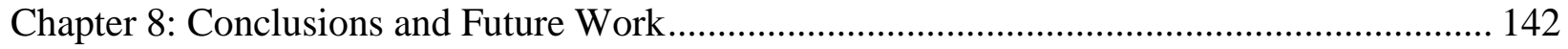

Appendix A: Physical Property Data (Standalone Models)................................................... 145

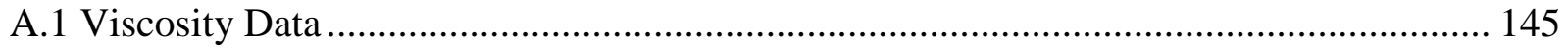

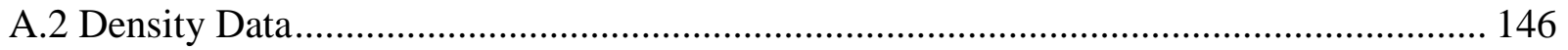

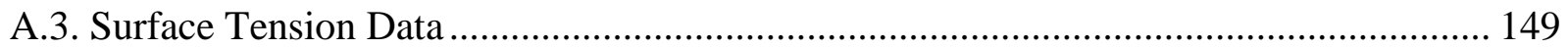

Appendix B: Physical Property Data (Thermodynamic Framework)....................................... 150

B.1 $\mathrm{CO}_{2}$ Partial Pressure ............................................................................................. 150 


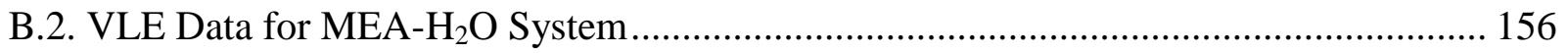

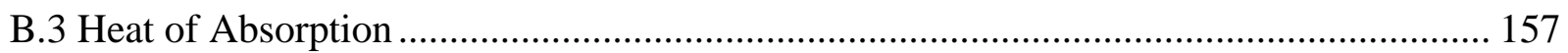

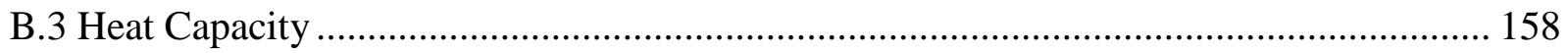

Appendix C. FORTRAN User Models Described in Work ………………………………….... 159

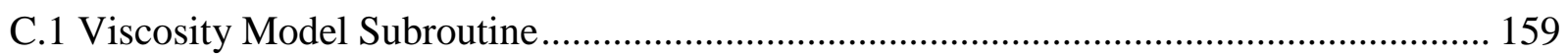

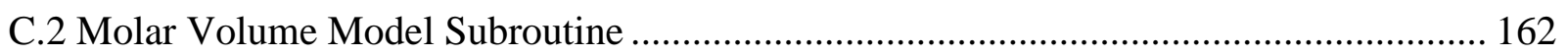

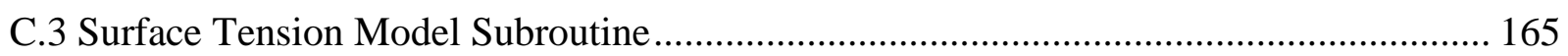

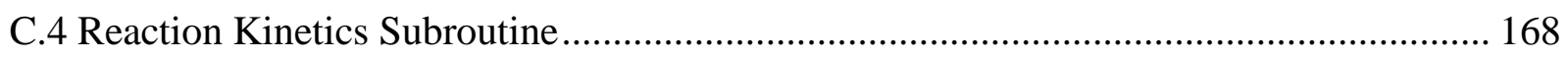

Appendix D: Additional Thermodynamic Model Results .................................................... 174

Appendix E: Liquid Solvent Ranges for Alternative Process Configurations............................. 181

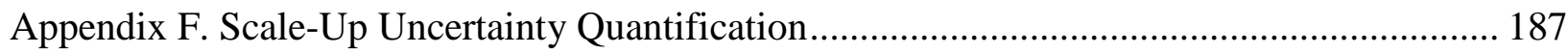

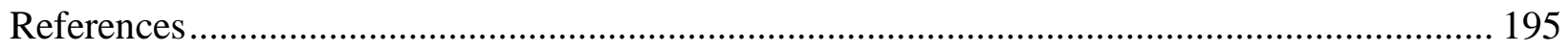




\section{List of Figures}

Figure 1-1. Chemical structure of monoethanolamine ………...................................................... 5

Figure 2-1. Overview of UQ for Physical Property Models ......................................................... 9

Figure 2-2. Schematic of process model uncertainty quantification in which parametric uncertainty from submodels is propagated through overall model to estimate uncertainty in process model variables.

Figure 3-1. Comparison of models to experimental data for solutions of: A) $20 \mathrm{wt} \% \mathrm{MEA}$; B) 30 wt\% MEA; C) $40 \mathrm{wt} \%$ MEA. Stars (*) represent experimental data from Amundsen et al. (2009), dashed lines represent model with parameters given by Weiland et al. (1998), and solid lines represent model with re-calibrated parameters.

Figure 3-2. Comparison of models to experimental data for density of solutions with: A) $20 \mathrm{wt} \%$ MEA; B) $30 \mathrm{wt} \%$ MEA; C) $40 \mathrm{wt} \%$ MEA. Stars (*) represent experimental data from sources listed in Table 3-3, dashed lines represent model from Weiland et al. (1998), and solid lines represent model developed in this work.

Figure 3-3. Comparison of models to experimental data for solutions of: A) $20 \mathrm{wt} \% \mathrm{MEA}$; B) 30 wt $\%$ MEA; C) 40 wt\% MEA. Stars (*) represent experimental data from Jayarathna et al. (2013), dashed lines represent original model, and solid lines represent new model.

Figure 4-1. Comparison of $\mathrm{CO}_{2}$ partial pressure data from various sources for solutions of 30 wt $\%$ MEA and temperatures of (A) $40^{\circ} \mathrm{C}$, (B) $80^{\circ} \mathrm{C}$, and (C) $120^{\circ} \mathrm{C}$ as a function of $\mathrm{CO}_{2}$ loading to new and existing models.

Figure 4-2. Comparison of phase envelope predictions to experimental data. T-xy data from Cai et al. (1996) are shown for fixed pressure of (A) $101.33 \mathrm{kPa}$ and (B) $66.66 \mathrm{kPa}$. P-xy data from Tochigi et al. (1999) are shown at fixed temperature of (C) $363.15 \mathrm{~K}$

Figure 4-3. Heat of absorption data (Kim et al., 2014) for solutions of $30 \mathrm{wt} \%$ MEA and temperatures of $40^{\circ} \mathrm{C}, 80^{\circ} \mathrm{C}$, and $120^{\circ} \mathrm{C}$ as a function of $\mathrm{CO}_{2}$ loading compared to model predictions for (A) CCSI, (B) Plaza, and (C) Aspen Plus library models.

Figure 4-4. CCSI model and Plaza model predictions of heat capacity compared to data (Weiland et al., 1997) for solutions at $25^{\circ} \mathrm{C}$ for various values of MEA weight percent as a function of $\mathrm{CO}_{2}$ loading 42

Figure 5-1. Response surfaces for viscosity model

Figure 5-2. Approximation of (A) cumulative distribution functions and (B) probability distribution functions for viscosity models parameters determined from confidence intervals in the deterministic regression results 
Figure 5-3. Posterior distributions for viscosity model parameters obtained from Bayesian inference. (A) Histograms representing marginal distributions of the parameters; (B) contour plot representing joint distribution.

Figure 5-4. Stochastic viscosity model for $\mathrm{W}_{\mathrm{MEA}}=30 \%$ considering $(\mathrm{A})$ prior parameter distribution and (B) posterior parameter distribution. Experimental data represented by (*) and taken from Amundsen et al. (2009)

Figure 5-5. Approximations of (A) cumulative distribution functions and (B) probability distribution functions for density model parameters determined from the confidence intervals in the deterministic regression results.

Figure 5-6. Posterior distributions for density model parameters obtained from Bayesian inference. (A) Histograms representing single parameter marginal distributions of the parameters; (B) Contour plots representing two parameter marginal distributions. 55

Figure 5-7. Approximations of (A) cumulative distribution functions and (B) probability distribution functions for surface tension model parameters determined from the confidence intervals in the deterministic regression results.

Figure 5-8. Posterior distributions for surface tension model parameters obtained from Bayesian inference. (a) Histograms representing single parameter marginal distributions of the parameters; (B) Contour plots representing two parameter marginal distributions. 58

Figure 5-9. $\mathrm{CO}_{2}$ partial pressure prediction for stochastic model with a modified prior parameter distribution with experimental data for $30 \mathrm{wt} \% \mathrm{MEA}$ at temperatures of (a) $40^{\circ} \mathrm{C}$, (b) $80^{\circ} \mathrm{C}$, and (c) $120^{\circ} \mathrm{C}$

Figure 5-10. Quality of response surface model for $\mathrm{CO}_{2}$ partial pressure. (A) Parity plot for cross validation (10-fold) of response surface model in comparison to simulation values. Values shown correspond to natural logarithm of $\mathrm{CO}_{2}$ partial pressure (B) Ratio of response surface predictions to simulation values for $\mathrm{CO}_{2}$ partial pressure (without logarithmic transformation) as a function of $\mathrm{CO}_{2}$ loading.

Figure 5-11. Single parameter marginal probability density functions for prior and posterior distributions. Red and blue lines represent prior and posterior distributions, respectively, and parameter numbers match those given in Table 4.4. Parameter values are normalized by dividing by the deterministic value of the respective parameter.

Figure 5-12. Normalized prior and posterior two-parameter marginal distributions for (A) parameters 1 and 2 and (B) parameters 5 and 6 .

Figure 5-13. $\mathrm{CO}_{2}$ partial pressure prediction for stochastic model with a posterior parameter distribution with experimental data for $30 \mathrm{wt} \% \mathrm{MEA}$ at temperatures of (a) $40^{\circ} \mathrm{C}$, (b) $80^{\circ} \mathrm{C}$, and (c) $120^{\circ} \mathrm{C}$ 
Figure 5-14. Estimated pdfs for absorber $\mathrm{CO}_{2}$ capture percentage resulting from propagating parametric uncertainty in viscosity, molar volume, and surface tension models through process model.

Figure 5-15. Correlations between (A) viscosity, (B) density, and (C) surface tension in the lean solvent stream and $\mathrm{CO}_{2}$ capture percentage of the absorber column. Variation in physical properties attributed to propagating prior parameter distributions through the process model for all three physical properties.

Figure 5-16. Pdfs for $\mathrm{CO} 2$ capture percentage for stochastic absorber models for (A) Case 1 and (B) Case 2

Figure 5-17. Pdfs for predicted lean solvent loading for stochastic regenerator models for (A) Case 1 and (B) Case 2

Figure 6-1. Simultaneous variation of flowrates in proposed test matrix for (A) flue gas flowrate vs. reboiler steam flowrate, (B) solvent flowrate vs. reboiler steam flowrate, and (C) solvent flowrate vs. flue gas flowrate.

Figure 6-2. Parity plot and residual plot for gas side $\mathrm{CO}_{2}$ capture predicted by absorber model. 81

Figure 6-3. Comparison of model and data absorber temperature profiles for Cases K1-K12. Relative column positions of 0 and 1 represent top and bottom of column, respectively........... 82

Figure 6-4. Comparison of model and data absorber temperature profiles for Cases K13-K23. Relative column positions of 0 and 1 represent top and bottom of column, respectively.......... 83

Figure 6-5. Values of temperature profile error (Eqn. 6.1) calculated for absorber column simulations

Figure 6-6. Comparison of experimental data and model prediction of $\mathrm{CO}_{2}$ loading in the regenerator solvent outlet.....

Figure 6-7. Comparison of model and data stripper temperature profiles for Cases K1-K12. Relative column positions of 0 and 1 represent top and bottom of column, respectively. Red and blue lines represent simulation results for complete process model and individual regenerator model, respectively.

Figure 6-8. Comparison of model and data stripper temperature profiles for Cases K13-K23. Relative column positions of 0 and 1 represent top and bottom of column, respectively. Red and blue lines represent simulation results for complete process model and individual regenerator model, respectively. 88

Figure 6-9. Values of temperature profile error (Eqn. 6.1) calculated for stripper column simulation. Blue squares represent case in which stripper column is simulated separately and red squares represent combined absorber/stripper simulation. 89 
Figure 6-10. Estimated pdfs of $\mathrm{CO}_{2}$ capture percentage resulting from propagating physical property model parameters through process model

Figure 6-11.Estimated pdfs of output lean solvent loading resulting from propagating physical property model parameters through process model

Figure 6-12. Estimated pdfs for single parameter marginal distributions of mass transfer model parameters considered for UQ.

Figure 6-13. Estimated pdfs for single parameter marginal distributions of hydraulic model parameters considered for UQ....

Figure 6-14. Estimated pdfs resulting from propagating parametric uncertainty through process model for three cases. Pdfs for $\mathrm{CO}_{2}$ capture percentage given in (A) and predicted lean loading in (B). Red lines represent case in which thermodynamic model parameter uncertainty is considered and blue lines represent case in which thermodynamic, mass transfer, and hydraulics model uncertainty is considered.

Figure 6-15. Calculated temperature profiles for absorber column stochastic model. (A) Deterministic model temperature profile represented by black line and temperature profiles calculated for realizations from parameter distribution given by blue lines (B) Estimated pdfs for average temperature profile error for stochastic simulation. Red lines represent case in which thermodynamic model parameter uncertainty is considered and blue lines represent case in which thermodynamic, mass transfer, and hydraulics model uncertainty is considered. 98

Figure 6-16. Calculated temperature profiles for stripper column stochastic model. (A) Deterministic model temperature profile represented by black line and temperature profiles calculated for realizations from parameter distribution given by blue lines (B) Estimated pdfs for average temperature profile error for stochastic simulation. Red lines represent case in which thermodynamic model parameter uncertainty is considered and blue lines represent case in which thermodynamic, mass transfer, and hydraulics model uncertainty is considered.

Figure 6-17. Values of Sobol indices for parameters with respect to $\mathrm{CO}_{2}$ capture percentage prediction for three cases 101

Figure 6-18. Values of Sobol indices for parameters with respect to lean loading prediction for three cases 101

Figure 6-19. Results of sensitivity study for Case K6 in which effect of the measurement error assumption on calculated values of $\mathrm{CO}_{2}$ capture percentage in absorber and lean loading in stripper outlet is analyzed. Solid blue lines represent calculated values over measurement error ranges and blue squares used to highlight baseline model values. Dashed black lines used to denote data values of process variables (horizontal) and estimated average values of measurement error (vertical). Results are given for perturbations in (A) $\mathrm{CO}_{2}$ weight percentage and (B) MEA weight percentage.

Figure 6-20. Results of sensitivity study in which predictions of $\mathrm{CO}_{2}$ capture and stripper outlet lean loading are analyzed as a function of assumed measurement error in $\mathrm{CO}_{2}$ weight percentage. 
Solid blue lines represent calculated values over measurement error ranges and blue squares used to highlight baseline model values. Dashed black lines used to denote data values of process variables (horizontal) and estimated average values of measurement error (vertical). .............. 106

Figure 6-21. Parity plots for (A) $\mathrm{CO}_{2}$ capture percentage and (B) NTU for absorber column simulation with and without assumption of $\mathrm{CO}_{2}$ concentration discrepancy. 107

Figure 6-22. Comparison of TCM absorber temperature profiles for two cases........................ 109

Figure 6-23. Comparison of TCM stripper temperature profiles for two cases.......................... 110

Figure 6-24. Comparison of TCM absorber lean loading profiles for two cases ........................ 111

Figure 7-1. Schematic of proposed Bayesian DOE for MEA campaign at NCCC .................... 114

Figure 7-2. Effect of lean solvent flowrate on $\mathrm{CO}_{2}$ capture percentage in absorber with 3 beds and intercooling, with variable $\mathrm{CO}_{2}$ loading in solvent and $\mathrm{CO}_{2}$ weight fraction in flue gas.... 116

Figure 7-3. Effect of lean solvent flowrate, flue gas flowrate, and lean solvent loading on $\mathrm{CO}_{2}$ capture percentage of absorber. $\mathrm{CO}_{2}$ weight percent in flue gas is fixed at $15 \% \ldots \ldots \ldots \ldots \ldots \ldots \ldots \ldots . .117$

Figure 7-4. Effect of liquid to gas (L:G) ratio, flue gas flowrate, and lean solvent loading on $\mathrm{CO}_{2}$ capture percentage of absorber ………………………….................................................. 118

Figure 7-5. Estimated ranges of liquid flowrate, for a given value of lean loading and $12.5 \mathrm{wt} \%$ $\mathrm{CO}_{2}$ in flue gas, for which $\mathrm{CO}_{2}$ capture percentage is constrained between $50-95 \%$. Solid lines represent the ranges of liquid flowrate for which requirement is met, and dashed line is included to illustrate additional constraint that liquid flowrate remains above $3000 \mathrm{~kg} / \mathrm{hr}$.

Figure 7-6. Estimated ranges of liquid flowrate, for a given value of lean loading and $15 \mathrm{wt} \%$ $\mathrm{CO}_{2}$ in flue gas, for which $\mathrm{CO}_{2}$ capture percentage is constrained between 50-95\%. Solid lines represent the ranges of liquid flowrate for which requirement is met, and dashed line is included to illustrate additional constraint that liquid flowrate remains between $3000-13000 \mathrm{~kg} / \mathrm{hr}$. ...... 121

Figure 7-7. Estimated ranges of liquid flowrate, for a given value of lean loading and $17.5 \mathrm{wt} \%$ $\mathrm{CO}_{2}$ in flue gas, for which $\mathrm{CO}_{2}$ capture percentage is constrained between 50-95\%. Solid lines represent the ranges of liquid flowrate for which requirement is met, and dashed line is included to illustrate additional constraint that liquid flowrate remains between $3000-13000 \mathrm{~kg} / \mathrm{hr}$...... 122

Figure 7-8. Sensitivity study in which the required reboiler duty to reduce the lean solvent loading to a given value is calculated as a function of rich solvent flowrate and $\mathrm{CO}_{2}$ loading. 124

Figure 7-9. Estimated widths of $95 \%$ confidence intervals in absorber efficiency as a function of lean solvent flowrate, flue gas flowrate, and lean loading. Comparison given for a fixed $\mathrm{CO}_{2}$ weight percentage in flue gas (15\%). Squares represent discrete points for which the confidence intervals are calculated, and the lines are included to improve visibility of the trends. 125 
Figure 7-10. Estimated widths of $95 \%$ confidence intervals in absorber efficiency as a function of $\mathrm{CO}_{2}$ capture percentage, flue gas flowrate, and lean loading. Comparison given for a fixed $\mathrm{CO}_{2}$ weight percentage in flue gas $(15 \%)$. Squares represent discrete points for which the confidence intervals are calculated, and the lines are included to improve visibility of the trends.

Figure 7-11. Parity plot for comparison of $\mathrm{CO}_{2}$ capture percentage predicted by original Aspen Plus model and surrogate response surface model. 128

Figure 7-12. Graphical comparison of first set of experiments for 2017 campaign (black dots) and 2014 campaign (red dots)..... 130

Figure 7-13. Parity plot for comparison of model prediction of $\mathrm{CO}_{2}$ capture percentage to experimental data, for operation with three beds and intercooling.

Figure 7-14. Estimated marginal pdfs for prior (red) and posterior (blue) distributions of parameters updated in Bayesian inference with $\mathrm{CO}_{2}$ capture percentage data

Figure 7-15. Effect of incorporating absorber efficiency data into Bayesian estimation of mass transfer and hydraulics model parameters. Confidence interval widths, as calculated by the surrogate absorber model, are shown for (A) grid of 448 points spread throughout input space and (B) points for which experimental data are collected.

Figure 7-16. Parity plot for $\mathrm{CO}_{2}$ capture percentage in absorber ( 3 beds with intercooling configuration) for complete set of NCCC data.

Figure 7-17. Estimated marginal pdfs for prior (red) and posterior (blue) distributions of parameters for second round of Bayesian DOE.

Figure 7-18. Change in estimated width of $95 \%$ confidence interval in $\mathrm{CO}_{2}$ capture percentage for points in which data were collected in second round of Bayesian DOE.

Figure 7-19. Parity plot for $\mathrm{CO}_{2}$ capture percentage predicted by model and experimental data for cases in which absorber is operated with one or two beds. 


\section{List of Tables}

Table 3-1. Comparison of original and calibrated parameter values for the solution viscosity model

Table 3-2. Parameter values for Weiland et al. (1998) solution molar volume model................ 16

Table 3-3. Summary of density data applied to model regression......................................... 18

Table 3-4. Calibrated parameter values for molar volume model given in Eq. 3.13................. 18

Table 3-5. Values of average absolute relative deviation for original and new density models .. 19

Table 3-6. MEA composition dependent parameters for surface tension model....................... 21

Table 3-7. MEA composition dependent parameters for $\mathrm{CO}_{2}$ surface tension term .................. 21

Table 3-8. Parameters for pure component surface tension $(N / m)$ calculation......................... 22

Table 3-9. Parameters for updated $\mathrm{CO}_{2}$ surface tension term............................................... 23

Table 3-10. Regressed parameter values for new surface tension model ............................... 23

Table 4-1. Reaction kinetics parameters taken from Plaza (2012) ....................................... 32

Table 4-2. Regressed values of heat capacity (J/kmol-K) polynomial parameters................... 34

Table 4-3. Model AIC for varying number of NRTL parameters ......................................... 34

Table 4-4. Values of parameters included in thermodynamic framework.............................. 35

Table 4-5. Correlation coefficients of parameters in thermodynamic framework regression ...... 36

Table 4-6. $\mathrm{CO}_{2}$ partial pressure data used in thermodynamic framework regression ................. 38

Table 4-7. Values of SSE for models with respect to data included in the CCSI model regression

Table 4-8. Values of $\mathrm{R}^{2}$ for models with respect to data included in the CCSI model regression 43

Table 4-9. ANOVA table for regression $\left(\mathrm{CO}_{2}\right.$ Partial Pressure $)$........................................... 44

Table 4-10. ANOVA table for regression (Heat Capacity) ................................................... 44

Table 4-11. ANOVA table for regression (Heat of Absorption) ........................................... 44

Table 5-1. Calculated 95\% confidence intervals for parameters in viscosity model.................. 50 
Table 5-2. Calculated $95 \%$ confidence intervals for parameters in molar volume model........... 53

Table 5-3. Calculated $95 \%$ confidence intervals for parameters in surface tension model ......... 56

Table 5-4. Input variables for absorber simulation in case study with viscosity, molar volume,

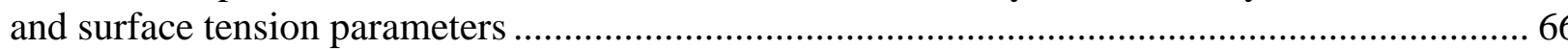

Table 5-5. Results of propagating prior and posterior distributions of viscosity, molar volume, and surface tension parameters though absorber simulation

Table 5-6. Input variables for stripper simulation in case study with viscosity, molar volume, and surface tension parameters 70

Table 5-7. Resulting of propagating prior and posterior distributions of viscosity, molar volume, and surface tension parameters through stripper column 71

Table 5-8. Input variables for absorber simulation in case study with thermodynamic parameters

Table 5-9. Input variables for absorber simulation in case study with thermodynamic parameters

Table 6-1. Summary of NCCC steady state data (Absorber variables) .................................... 78

Table 6-2. Summary of NCCC steady state data (Stripper variables) ................................... 79

Table 6-3. Comparison of model predictions of $\mathrm{CO}_{2}$ capture percentage with data values calculated from the liquid and gas side mass balances

Table 6-4. Comparison of model predictions of $\mathrm{CO}_{2}$ loading in the outlet lean solvent from the regenerator

Table 6-5. List of physical property model parameters for which distribution are included in stochastic model.

Table 6-6. Results of Sobol sensitivity analysis for NCCC absorber and stripper model for three

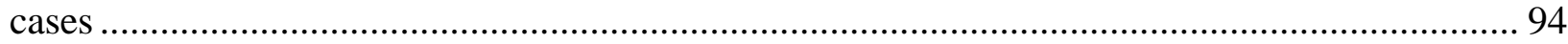

Table 6-7. Mass transfer model parameters considered for UQ ........................................... 95

Table 6-8. Hydraulics model parameters considered for UQ ............................................ 95

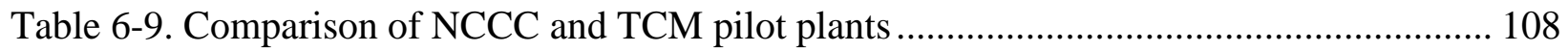

Table 6-10. Process variable values for two TCM test cases ............................................. 109

Table 7-1. First set of cases selected for 2017 MEA test campaign at NCCC ....................... 129

Table 7-2. Results of three beds with intercooling test for first test plan ............................. 131 
Table 7-3. Test plan for second round of sequential DOE

Table 7-4. Experimental data for second round of sequential DOE.

Table 7-5. Results of one bed absorber test ..................................................................... 139

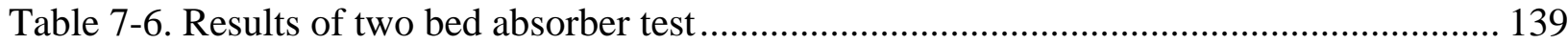




\section{Chapter 1 : Introduction}

\subsection{Research Motivation}

Due to concern over the role of increasing fossil fuel emissions on climate change, much research has been focused on developing new processes and improving the existing technologies for carbon capture and storage (CCS). CCS refers to a wide range of technology-based strategies for reducing the atmospheric emissions of fossil fuels at point sources and safe storage of the greenhouse gases (Gibbins and Chalmers, 2008). Although the use of renewable energy has become widespread, fossil fuel combustion still accounts for approximately $85 \%$ of industrial energy use; accordingly, CCS is considered as an essential part of the transition away from fossil fuels in the near future (Haszeldine, 2009). The costs of existing CCS technologies, however, are large enough to inhibit widespread deployment at power plants (Rubin et al., 2015). Accordingly, the development of new CCS strategies as well as improvement of existing technologies remains a major topic for research. An outline of technologies for carbon capture and a review of ongoing research are given in the work of Boot-Handford et al. (2014). Some of the technologies highlighted here include solvent-based $\mathrm{CO}_{2}$ capture strategies, including both traditional amine systems and novel solvent systems such as ionic liquids. Oxyfuel, chemicallooping, and calcium combustion are also discussed, as are other technologies including solid sorbent systems and direct air capture technology.

The United States Department of Energy's Carbon Capture Simulation Initiative (CCSI) has a strong focus on the development of state of the art process models and computational tools for accelerating the development and commercialization of post-combustion carbon capture system technologies (Miller et al., 2014). One of the process models that has been developed under the auspices of CCSI is a model that will serve as a definitive reference for benchmarking the performance of solvent based $\mathrm{CO}_{2}$ capture systems. For developing this model, several requirements must be met. All physical property models must be developed with parameters calibrated to fit lab-scale and bench-scale data for the entire range of operating conditions of $\mathrm{CO}_{2}$ absorption and solvent regeneration processes, namely temperature and composition of the solvent and gas streams and operating pressure. It is difficult to measure some of the properties, such as diffusivity, especially for chemical solvents in which reactions take place simultaneously 
with mass transfer. For such properties, hydraulic and mass transfer models should be developed simultaneously with the physical property models on which they depend and also validated using bench scale data. Reaction kinetics for chemical solvent systems, which are coupled with the system thermodynamic framework, must also be quantified. The model should also include a framework for uncertainty quantification (UQ), which provides a methodology for estimating uncertainties in process variables due to uncertainty in the submodels. The overall process model, which is developed from the aforementioned submodels, must be validated with both steady state and dynamic process data over the operational ranges of industrial interest. When the model is used to estimate the outputs from a pilot plant or a commercial process, the model discrepancies should be identified and be accounted for by developing additional models and uncertainty quantification methodologies. Such analysis can help in identifying scale-up uncertainties and eventually lead to a rigorous approach to quantify such uncertainties.

This work is focused on two major aspects of the model development, namely physical property modeling with uncertainty quantification for the aqueous monoethanolamine (MEA) $\mathrm{CO}_{2}$ capture system and development of a steady state process model. Validation data are obtained from the National Carbon Capture Center (NCCC) in two separate test campaigns, the second of which is

planned with a novel Bayesian design of experiments (DOE) methodology that is described in this work. Some background, including literature review, on the topics of general physical property modeling with UQ and the MEA-based $\mathrm{CO}_{2}$ capture system is presented in the succeeding sections of this chapter.

\subsection{Physical Property Modeling with Uncertainty Quantification}

The predictive capability of rigorous process models is dependent on the accuracy of the underlying physical property models. Since uncertainty in physical property models is unavoidable, it is important that these uncertainties are quantified. Most physical property models used in process simulation are deterministic in nature, indicating that input variables and model parameters, and thus the model outputs, have fixed point values, and uncertainty is not taken into consideration. For physical property models, the input (predictor) variables generally include temperature, pressure, and composition. The model form and the parameter values, which are generally calibrated to fit experimental data, dictate the mapping of the input variables to predictions of one or more physical property values of interest. 
In contrast to deterministic models, stochastic models take uncertainty into consideration so that probability distributions of process output variables may be predicted. In the work of Whiting et al. (1996), four types of uncertainty are highlighted, particularly with respect to thermodynamic property models. Model form uncertainty is based on the idea that most property models implemented into process simulators generally contain simplifications even when based upon first principles, whereas many use simple empirical correlations. This uncertainty is generally not quantified when a single model is chosen without applying a rigorous model selection methodology. Model parameter uncertainty is based on the fact that exact point values of model parameters may not always be estimated, and the parameters may more appropriately be quantified in terms of a probability distribution over a range of possible values. Experimental data uncertainty generally needs to be considered because the data for which model parameters are calibrated to fit generally are likely to be measured with some imprecision or bias. Finally, process simulator uncertainty is related to the tendency to get different results when using various simulators to solve a problem due to differences in tolerances and algorithms.

Sources of uncertainty in modeling may generally be characterized as aleatoric or epistemic (Der Kiureghian and Ditlevsen, 2007). Aleatoric uncertainty is generally associated with phenomena that are naturally random, so that uncertainty of this type is of fixed value. In contrast, epistemic uncertainty is attributed to a lack of knowledge in a process of interest, and is theoretically a function of the information available. For the physical property models considered in this work, all uncertainty is assumed to be epistemic. Although the actual values of the physical properties are fixed for a set of state variables, uncertainty arises in actual process data due to measurement inaccuracy or imprecision. Similarly, the corresponding parametric uncertainty is considered epistemic as it is quantified in a Bayesian framework in which an initial estimate of the uncertainty may be updated by taking experimental data into consideration.

Uncertainty analysis is often not considered in chemical engineering applications, mainly due to lack of systematic approaches for UQ and capability of propagating estimated uncertainty through a process model. One major challenge to the incorporation of UQ into models is the large parameter space present in complex models, and the frequent lack of data for adequately characterizing the model parameters (Papadimitriou, 2014). Some work has been focused on analyzing the sensitivity of process models to physical property parameters. For example, the work of Macchietto et al. (1986) is based on an approach of incorporating analytic derivatives 
into a flowsheet simulator for performing sensitivity analyses. Recently, many commercial process simulators have provided options for Monte Carlo simulation for use in uncertainty analysis (Kim et al., 2013; Mathias, 2014; Turton et al., 2012). The use of UQ methods in property and process modeling has increased in recent years due to computational advances, and some examples from the open literature are summarized here. The work of Whiting (1996) provides an overview of UQ and simple examples of strategies for quantification of thermodynamic model uncertainties involving Monte Carlo simulation, regression analysis, and optimization. A Monte Carlo approach has been applied to study the sources of uncertainty in reaction kinetics of a coal devolatilization process (Gel et al., 2014). Multiple studies on UQ of multiphase computational fluid dynamics (CFD) of fluidized beds have been performed (Gel et al., 2013; Lane et al., 2014). A rigorous approach to UQ of the thermodynamics of a solid sorbent-based $\mathrm{CO}_{2}$ capture process has been developed (Mebane et al, 2013). Perturbation methods have been used to study the effects of such factors as chemical equilibrium, reaction kinetics, activity coefficient models, and viscosity on process simulations, including $\mathrm{CO}_{2}$ capture applications (Mathias and Gilmartin, 2014; Mathias, 2014). Weber et al. (2006) used a Bayesian approach to study thermodynamic uncertainty on the chemical speciation in river water. Mišković and Hatzimanikatis (2011) have developed a methodology in which the uncertainty in enzyme kinetics is modeled and the responses of metabolic reactions to changes in enzyme activities under uncertainty are predicted. A study by Sarkar et al. (2012) involves Bayesian calibration of thermodynamic parameters for geochemical speciation.

\subsection{Carbon Capture with Aqueous MEA System}

Monoethanolamine has been utilized as a reactive solvent for absorption since 1930, and aqueous solutions with $30 \mathrm{wt} \%$ MEA have been the industrial standard for acid gas removal since 1970 (Amundsen et al., 2009). Alkanolamines such as MEA are extensively used in $\mathrm{CO}_{2}$ capture applications due to their chemical structure, which includes hydroxyl groups that increase water solubility and amino groups that provide the alkalinity necessary to capture $\mathrm{CO}_{2}$ (Jayarathna et al., 2013). The chemical structure of monoethanolamine is represented in Figure 1.1. 


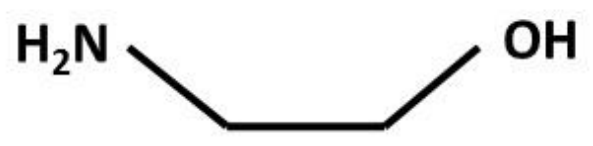

Figure 1-1. Chemical structure of monoethanolamine

Physical property models for this system, including the thermodynamic framework, have also been reported in the open literature, although without consideration of uncertainty quantification. Frameworks using the e-NRTL and UNIQUAC models are available in the literature (Hilliard, 2008; Plaza, 2012; Zhang et al., 2011; Aspen Plus documentation, 2013a; Aronu et al., 2011; Kaewsichan et al., 2001; Vrachnos et al., 2006). Deterministic process models of the $\mathrm{CO}_{2}$ capture system with MEA are also widely available in the open literature, including validation with pilot plant data. Most of the existing validation work, however, is based on small scale pilot plants and over narrow operating ranges. In the work of Luo et al. (2009), models from leading commercial simulators are compared with data from four pilot plants, with significant discrepancy in the results generated by the various models for both absorber and stripper operation. Previous MEA solvent test campaigns have been performed and used for model validation at the pilot plant at the J.J. Pickle Research Center of The University of Texas at Austin (Plaza, 2012; Van Wagener, 2011; Zhang et al., 2009). The pilot plant at SaskPower's Boundary Dam Power Station has also been the subject of previous validation work with MEA (Wilson et al., 2004; Idem et al., 2006). Data from the pilot plant at the University of Kaiserslautern have been reported for various solvent systems, with aqueous MEA generally considered as a baseline (Mangalapally et al., 2009, 2012; Mangalapally and Hasse, 2011a-c; Notz et al., 2012; Zhang and Chen, 2013). Multiple papers from Moser et al. (2011a-c) have presented information regarding a long-term MEA test at RWE Power's power station in Niederaussem, Germany. In addition to those mentioned here, various other MEA model validation studies with small-scale pilot plant data have been reported in the open literature (Saimpert et al., 2013; Han, Graves, Neathery, \& Kunlei, 2011; Sønderby et al., 2013; Tobiesen et al., 2007, 2008; Artanto et al, 2012; Faramarzi et al., 2010; Kwak et al., 2012; Rabensteiner et al., 2014; Simon et al., 2011). Some work on MEA model validation with large-scale pilot plant 
data is available in the open literature for the CASTOR project pilot plant at the Esbjerg power station in Denmark, although validation over a large range of operating conditions for both columns is not shown (Knudsen et al., 2009; Dugas et al, 2009).

Prior to the development of the MEA model referred to in this work, all known existing models of this process available in the literature were deterministic in nature, meaning that rigorous parametric uncertainty quantification had not been considered. However, some work has been performed with respect to studying the sensitivity of process models for this system to process variables as well as parameters for the underlying submodels. In the work of Nuchitprasittichai and Cremaschi (2013), a sensitivity study is performed in which the effect of fluctuation in glue gas $\mathrm{CO}_{2}$ concentration and utility costs on process economics is studied for various amine-based $\mathrm{CO}_{2}$ capture systems. In the work of Tönnies et al. (2011), a sensitivity study is performed for the MEA system with respect to input parameters for fluid dynamics and physical and chemical properties of the system. In the MEA system absorber model validation work of Tobiesen et al. (2007), a parametric sensitivity study has been performed with the conclusion that the match between experimental data and model predictions are particularly sensitive to the equilibrium. In a work by deMontigny et al. (2001), the effects of operating variables such as liquid and gas flow, solvent $\mathrm{CO}_{2}$ loading and concentration, and $\mathrm{CO}_{2}$ partial pressure on the overall mass transfer coefficient are studied. An optimization study is performed by Dinca and Badea (2013) in which process variables such as solvent flowrate and composition and operating temperatures are manipulated to minimize heat required for MEA regeneration and maximize $\mathrm{CO}_{2}$ capture percentage. Another optimization study (Mores et al., 2011) focuses on maximizing the ratio between absorbed $\mathrm{CO}_{2}$ and total heating and cooling utility and the ratio between absorbed $\mathrm{CO}_{2}$ and amine flowrate by manipulating the key process variables.

\subsection{Scope of Research}

As mentioned previously, this dissertation describes select aspects in the development of a MEA solvent model, particularly with repsect to the physical property modeling and the validation of the complete process model with steady-state data from the National Carbon Capture Center (NCCC). The goal of the physical property modeling is to create a framework that is widely applicable to property modeling for any system, including uncertainty quantification. The contributions of this portion of the work are included in two manuscripts, for standalone property 
models such as viscosity, density, and molar volume of the $\mathrm{MEA}-\mathrm{CO}_{2}-\mathrm{H}_{2} \mathrm{O}$ system (Morgan et al., 2015) and the thermodynamic framework of this system. (Morgan et al., 2017a). The contributions of these works may be summarized as follows:

- Viscosity, molar volume, and surface tension models have been developed for the $\mathrm{CO}_{2}$ loaded aqueous MEA system, while including a novel parameter screening methodology based on a "sensitivity matrix" approach which involves analytical evaluations of derivatives of a physical property as a function of its input variables and parameters. A framework for uncertainty quantification is also developed and applied to each of these three models.

- Parameters for the vapor-liquid equilibrium (VLE), enthalpy, and chemistry models of the system are regressed together using data for binary $\left(\mathrm{MEA}-\mathrm{H}_{2} \mathrm{O}\right)$ and ternary $(\mathrm{MEA}-$ $\mathrm{H}_{2} \mathrm{O}-\mathrm{CO}_{2}$ ) VLE, heat of absorption, and heat capacity. These data are taken from nearly all sources of literature and correspond to temperature, pressure, and composition values that span the ranges of interest for the industrial $\mathrm{CO}_{2}$ capture process.

- The reaction kinetics of the system have been written in a form that ensures consistency with the thermodynamic model through inclusion of the chemical equilibrium constant. This makes use of a methodology outlined in the work of Mathias and Gilmartin (2014).

- A parameter screening methodology based on the information-theoretic criteria has been employed to determine and optimal set of parameters for inclusion in the final model.

- An approach for UQ of the thermodynamic framework, similar to that developed for the standalone physical property models, is employed for developing a stochastic model of the system.

The contributions of the steady-state validation project may be summarized as follows:

- A model of a large-scale pilot plant (Pilot Solvent Test Unit (PSTU) at NCCC) has been developed in Aspen Plus ${ }^{\circledR}$, consisting of physical property, kinetic, mass transfer, and hydraulic submodels developed as a component of the solvent model project. The physical property and kinetic models are discussed in this work, and the mass transfer and hydraulic models are detailed in another manuscript draft (Chinen et al., 2017).

- This model has been validated with experimental data that span a wide range of operating conditions, including liquid and gas flowrates and compositions, stripper reboiler duty, and packing height in the absorber column. 
- Probability density functions (pdfs) describing parametric uncertainty from the aforementioned submodels are propagated through the process model for various test cases, allowing estimation of the corresponding uncertainty in key process variables. The effect of uncertainty of individual parameters on the uncertainty in the process output variables is assessed for various operating conditions.

- Uncertainty in the measurement of the solvent composition has been experimentally determined, and the effect of the measurement uncertainty on the process output variables is assessed.

- In a second test campaign, a Bayesian design of experiments (DOE) methodology is being incorporated for planning the test campaign. An initial test plan has been created with the goal of including test runs throughout the input range of interest while selecting points that have relatively high uncertainty, as estimated from propagating parametric uncertainty through the process model. As experimental data are collected according to the test plan, the distributions of some of the model parameters are updated through Bayesian inference. The test plan is then updated based on the new estimates of the process uncertainty. The effectiveness of this process in reducing the estimated uncertainty in the absorber process model is demonstrated in this work.

The methodology for the development of the rigorous solvent model has been applied to a novel solvent system in a collaborative project with an industry partner. All process submodels have been developed from bench-scale data and incorporated into a process model, which has been validated on both bench-scale and pilot plant-scale (NCCC) with satisfactory results. Due to the proprietary nature of this project, the information is not detailed in this dissertation, although it is planned to be summarized in a forthcoming manuscript. 


\section{Chapter 2 : Property Modeling Overview}

\subsection{Outline}

In this work, deterministic and stochastic models are developed for physical properties, and a general overview of the methodology used to develop the models is presented here. For the deterministic model, the model output $(\varphi)$ is represented as a function of a set of predictor variables $(\tilde{x})$ and a set of model parameters $(\tilde{\theta})$, denoted by:

$$
\varphi=F(\tilde{x}, \tilde{\theta})
$$

The model parameters are calibrated by fitting the model form to $M$ experimental data, denoted by:

$$
Z=Z_{j}\left(\tilde{x}_{j}\right) \quad j=1,2, \ldots, M
$$

After developing the deterministic model, the stochastic model is created through the methodology shown schematically in Figure 2.1.

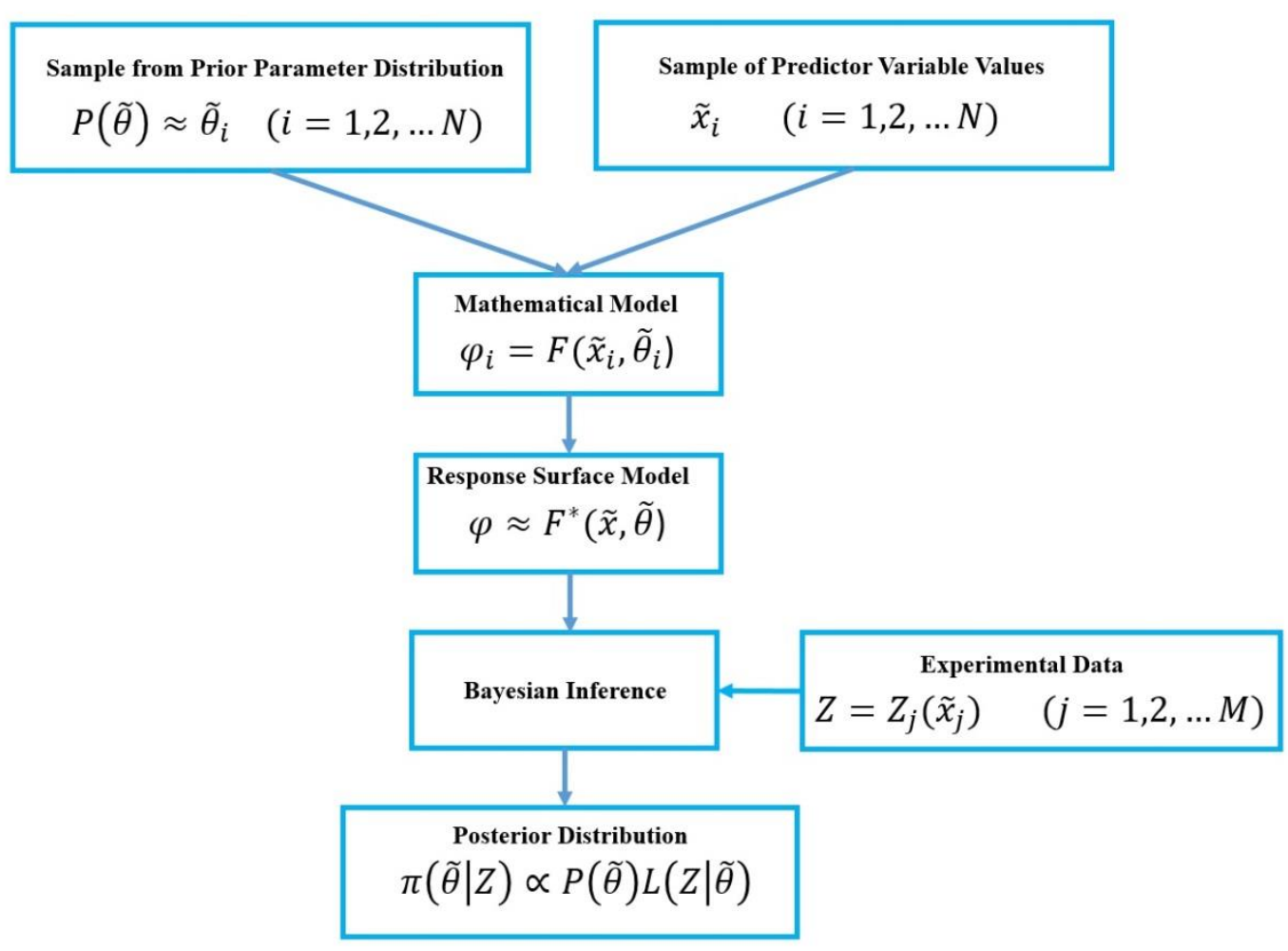

Figure 2-1. Overview of UQ for Physical Property Models 
In the process of Bayesian inference, experimental data are used to update beliefs about the distribution of a set of parameters. A prior distribution is an initial distribution based on one's previous beliefs about the parameters. A posterior distribution is derived through Bayesian inference as an updated belief that takes the experimental data into account as evidence. In this work, a joint prior distribution, denoted as $P(\tilde{\theta})$, is assumed a sample $\tilde{\theta}_{i}$ of size $N$ is taken. A sample $\tilde{x}_{i}$ of the predictor variables of interest is combined with the parameter sample, and the mathematical model is used to calculate the physical property value $\left(\varphi_{i}\right)$ for each set of parameters and predictor variables. A response surface model is created to map the input values of the parameters and predictor variables to the output physical property value, which results in a reduction in the computational cost of Bayesian inference. The function is replaced by an emulator, denoted as:

$$
\varphi=F^{*}(\tilde{x}, \tilde{\theta})
$$

In this work, a popular curve fitting method called multivariate adaptive regression splines (MARS) (Friedman, 1991) is used to develop the response surfaces Since thousands, if not more, simulations are required to estimate the posterior distributions, the use of surrogate response surface models in Bayesian inference applications is common practice, and many examples are available in the literature (e.g. Balakrishnan et al., 2003; Ma and Zabaras, 2009; Sudret, 2012; Bontinck et al., 2016). The advantages of response surface based uncertainty quantification and the risks associated with inaccurate surrogate models, particularly those developed from an inadequate sample of true model observations, are described in the work of Giunta et al. (2006). Cross validation is used to assess the response surface quality, specifically to ensure that the surrogate response surface model accurately represents the actual model without overfitting. The response surface model is expected to mimic the actual simulation model accurately, provided the cross validation test gives good results. The posterior distribution is computed by:

$$
\pi(\tilde{\theta} \mid Z) \propto P(\tilde{\theta}) L(Z \mid \tilde{\theta})
$$

where $P(\tilde{\theta})$ represents the prior distribution of the parameters, $\pi(\tilde{\theta} \mid Z)$ the posterior distribution, and $L(Z \mid \tilde{\theta})$ the likelihood function that measures the goodness of the match. The choice of prior distribution in this work varies for different physical properties, with more details given in the Chapter 5. The specific likelihood function used here is (Press et al., 1992): 


$$
L(Z \mid \tilde{\theta})=\exp \left(-0.5 \sum_{j=1}^{M} \frac{\left[F^{*}\left(\tilde{x}_{j}, \tilde{\theta}\right)-Z_{j}\left(\tilde{x}_{j}\right)\right]^{2}}{M \sigma_{j}^{2}}\right)
$$

The term $\sigma_{j}^{2}$ refers to the variance of an individual data observation $Z_{j}\left(\tilde{x}_{j}\right)$. This likelihood function is based on the chi-square statistic, a popular method for data fitting. It can be considered as a weighted least-squares method that gives more weight to more accurate data. It also assumes that there is no correlation between experiments and that variation of data from their true values follows a normal distribution. The posterior distribution is computed by the Markov Chain Monte Carlo (MCMC) method for which the likelihood function is evaluated many times in traversing the parameter space of $\theta$, and at each evaluation the response surface is also evaluated once (Beers, 2007). In the MCMC algorithm used in this work, the Gibbs sampling method is used to perform the search and has been determined to be adequate. The result of the Bayesian inference procedure is a joint posterior distribution of the parameters that is given in a form of a set of sample points.

Through this methodology, parametric uncertainty from various submodels can be estimated, and the overall process uncertainty may be estimated as shown schematically in Figure 2.2.

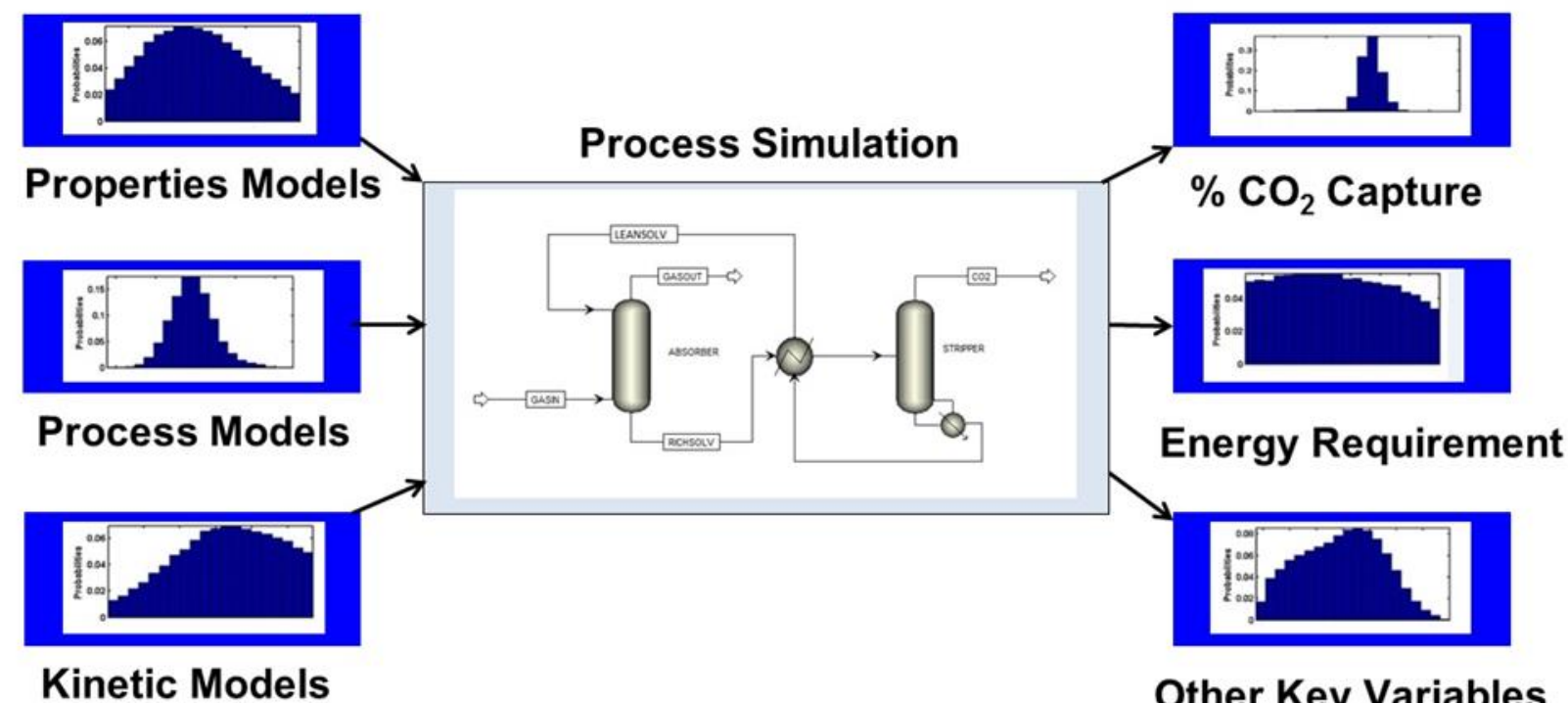

Figure 2-2. Schematic of process model uncertainty quantification in which parametric uncertainty from submodels is propagated through overall model to estimate uncertainty in process model variables. 
In Figure 2.2, the term "process models" is used to refer to submodels that are dependent on certain aspects of the process set-up, notably mass transfer and hydraulic models that are developed for a specific packing type. In contrast, the property models and kinetic models are valid for the $\mathrm{CO}_{2}$-loaded aqueous MEA system regardless of the process under consideration. Some process model uncertainties, which may be calculated in light of uncertainty in the submodels, include $\mathrm{CO}_{2}$ capture percentage for the absorber column and energy requirement to the reboiler of the stripper column, which is known to dictate the economic viability of a solventbased $\mathrm{CO}_{2}$ capture process.

\subsection{Conclusions}

In this section, a methodology for the complete development of an uncertainty quantification methodology for a solvent-based $\mathrm{CO}_{2}$ capture process has been presented. A system for developing physical property models, starting with a deterministic model fit to experimental data and eventually using a Bayesian inference-based uncertainty quantification procedure to develop a stochastic model. Finally, it is proposed that process model uncertainties may be quantified by propagating parametric uncertainty from the submodels, including physical property submodels, through the overall model. The development of deterministic physical property models is described in Chapters 3 (viscosity, molar volume, surface tension models) and 4 (thermodynamic framework). The uncertainty quantification of these models is described in Chapter 5, and the propagation of all submodel uncertainty throughout the overall model is described in Chapter 6. Although the mass transfer and hydraulic models are not developed directly as a part of this work, some information on their uncertainty quantification is given in Chapter 6.3.2. 


\section{Chapter 3 : MEA Physical Property Models (Deterministic)}

\subsection{Viscosity Model}

Empirical correlations for viscosity, molar volume, and surface tension have been adopted from the open literature and modified in this work to fit experimental data from various sources. For these models, the composition of the MEA- $\mathrm{CO}_{2}-\mathrm{H}_{2} \mathrm{O}$ system is represented in terms of the $\mathrm{CO}_{2}$ loading $(\alpha)$, the molar ratio of $\mathrm{CO}_{2}$ to MEA in solution, and the nominal weight fraction of MEA in aqueous solution $\left(w_{M E A}^{*}\right)$, defined on a $\mathrm{CO}_{2}$-free basis. For the viscosity model, this is represented in terms of a percentage, denoted as $W_{M E A}^{*}$. The temperature for all models is given in Kelvin.

The viscosity model is based on the model taken from Weiland et al. (1998), in which the solution viscosity $\left(\mu_{s l n}\right)$ is given as:

$$
\mu_{s l n}=\mu_{H_{2} O} \exp \left(\frac{\left(\left(a W_{M E A}^{*}+b\right) T+c W_{M E A}^{*}+d\right)\left(\alpha\left(e W_{M E A}^{*}+f T+g\right)+1\right) W_{M E A}^{*}}{T^{2}}\right)
$$

Fitting parameters for this model are denoted by $a$ - $g$. The viscosity of pure water $\left(\mu_{\mathrm{H}_{2} O} \mathrm{O}\right)$ is given (in mPa-s) as (Weast, 1984):

$$
\mu_{\mathrm{H}_{2} \mathrm{O}}=1.002 * 10^{\frac{1.3272\left(293.15-T-0.001053(T-293.15)^{2}\right)}{T-168.15}}
$$

Experimental data from Amundsen et al. (2009) with the variable ranges $W_{M E A}^{*} \in[20,40]$, $\alpha \in[0-0.5]$, and $T \in[298.15-353.15 \mathrm{~K}]$ are used to calibrate the parameters of Eq. 3.1. The values of the regressed parameters are compared to the values given by Weiland et al. (1998) in Table 3.1. 
Table 3-1. Comparison of original and calibrated parameter values for the solution viscosity model

\begin{tabular}{ccc}
\hline Parameter & $\begin{array}{c}\text { Value from Weiland et al. } \\
(2009)\end{array}$ & Value from this Work \\
\hline a & 0 & -0.0838 \\
b & 0 & 2.8817 \\
c & 21.186 & 33.651 \\
d & 2373 & 1817 \\
e & 0.01015 & 0.00847 \\
f & 0.0093 & 0.0103 \\
g & -2.2589 & -2.3890 \\
\hline
\end{tabular}

The predictions of the viscosity model, using both sets of parameters given in Table 3.1, are compared to the experimental data in Figure 3.1.

A

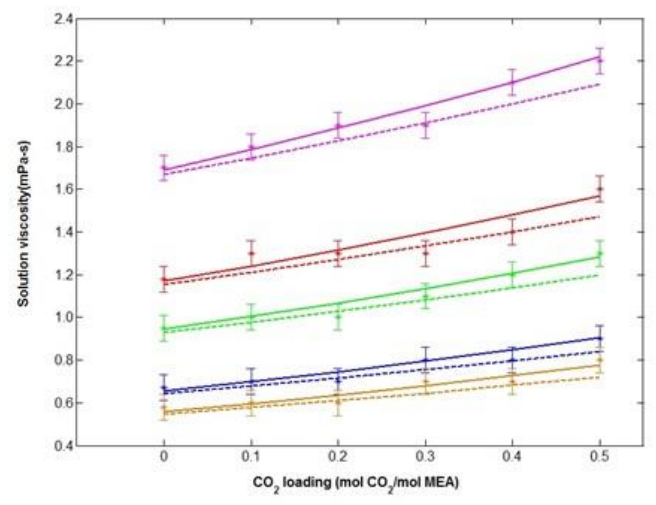

$\mathrm{C}$

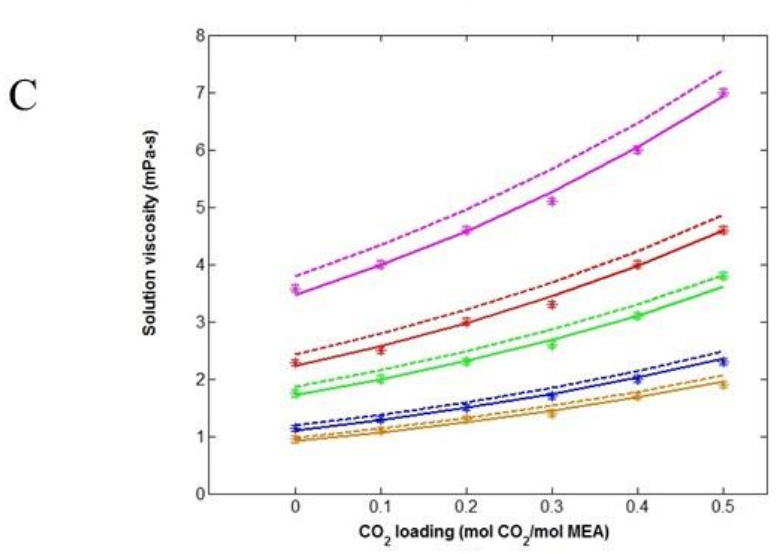

$\mathrm{B}$

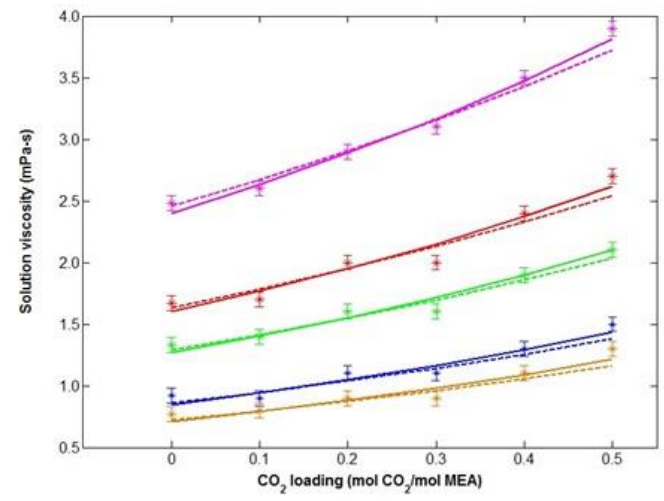

- $298.15 \mathrm{~K}$

- $313.15 \mathrm{~K}$

$=323.15 \mathrm{~K}$

- $343.15 \mathrm{~K}$

피 $353.15 \mathrm{~K}$

Figure 3-1. Comparison of models to experimental data for solutions of: A) 20 wt\% MEA; B) $30 \mathrm{wt} \%$ MEA; C) $40 \mathrm{wt} \%$ MEA. Stars (*) represent experimental data from Amundsen et al. (2009), dashed lines represent model with parameters given by Weiland et al. (1998), and solid lines represent model with re-calibrated parameters. 
By recalibrating the model parameters, the fit to the experimental data improved at relatively low temperatures and high amine concentrations, particularly for $W_{M E A}^{*}=40 \%$ and $T=298.15 \mathrm{~K}$. Although parameters $a$ and $b$ are assumed to be negligible in the works of Weiland et al. (1998) and Amundsen et al. (2009) for the MEA solvent, their inclusion in the model helps capture the effects of temperature and composition on the solution viscosity model more precisely. For higher temperatures and lower MEA weight percentage, there is little difference between the fit given by the model with the original and new sets of parameters. Overall, the absolute average relative deviation (AARD) of the fit of the viscosity model to the data decreases from $4.87 \%$ to $2.69 \%$ by re-calibrating the parameter set while including $a$ and $b$. The model form given in Eq. 3.1 is also tested with a 10 -fold cross validation and shown to be reasonable $\left(R^{2}=0.9972\right)$.

\subsection{Molar Volume Model}

\subsubsection{Summary of Existing Model}

The model for molar volume used in this work is also adapted from Weiland et al. (1998). In the original form, the solution molar volume $\left(V_{s l n}\right)$ is given by:

$$
\begin{aligned}
V_{\text {sln }}=x_{M E A} V_{M E A}+x_{\mathrm{H}_{2} \mathrm{O}} V_{\mathrm{H}_{2} \mathrm{O}} & +x_{\mathrm{CO}_{2}} V_{C O_{2}}+x_{M E A} x_{\mathrm{H}_{2} \mathrm{O}} V^{*}+x_{M E A} x_{C O_{2}} V^{* *} \\
V_{M E A} & =\frac{M W_{M E A}}{a T^{2}+b T+c} \\
V^{* *} & =d+e x_{M E A}
\end{aligned}
$$

The solution molar volume, as calculated in Eq. 3.3, consists of partial molar volume terms for the three components as well as terms that account for interaction between species. The terms $x_{i}$ and $V_{i}$ represent the mole fraction and molar volume, respectively, of species $i . V_{M E A}$ and $V_{\mathrm{H}_{2} \mathrm{O}}$ are pure component molar volume values, and $V_{\mathrm{CO}_{2}}$ represents the dissolved molar volume of $\mathrm{CO}_{2}$, which is a constant value and unrelated to the pure component value. The molar volume of MEA is calculated by Eq. 3.4 as a function of temperature. The molar volume associated with the interaction between $\mathrm{H}_{2} \mathrm{O}$ and MEA is given by $V^{*}$, a constant, and the molar volume associated with the interaction between MEA and $\mathrm{CO}_{2}$ is given by $V^{* *}$, which is dependent on 
the solution MEA concentration. The values of all constants used in this model are given in Table 3.2.

Table 3-2. Parameter values for Weiland et al. (1998) solution molar volume model

\begin{tabular}{cc}
\hline Parameter & Value \\
\hline $\mathrm{a}$ & $-5.35162 \times 10^{-7}$ \\
$\mathrm{~b}$ & $-4.51417 \times 10^{-4}$ \\
$\mathrm{c}$ & 1.19451 \\
$\mathrm{~d}$ & 0 \\
$\mathrm{e}$ & 0 \\
$V_{\mathrm{CO}_{2}}(\mathrm{~mL} / \mathrm{mol})$ & 0.04747 \\
$V^{*}(\mathrm{~mL} / \mathrm{mol})$ & -1.8218 \\
\hline
\end{tabular}

\subsubsection{Modified Model}

A new model for molar volume is developed by modifying the model given by Weiland et al. (1998), as described previously. The model is fit to experimental data for solution density, which is related to molar volume by:

$$
\rho_{s l n}=\frac{M W_{s l n}}{V_{s l n}}
$$

where $M W_{s l n}$ is the average of the molecular weights of the individual components. Whereas the previously described molar volume model does not account for the presence of the ionic species in the MEA- $\mathrm{CO}_{2}-\mathrm{H}_{2} \mathrm{O}$ system, the solution chemistry is taken into consideration in this work. The ionic speciation reactions for this system are taken from the work of Hilliard (2008) and given as:

$$
\begin{aligned}
& 2 \mathrm{MEA}+\mathrm{CO}_{2} \leftrightarrow \mathrm{MEA}^{+}+\mathrm{MEACOO}^{-} \\
& \mathrm{MEA}+\mathrm{CO}_{2}+\mathrm{H}_{2} \mathrm{O} \leftrightarrow \mathrm{MEA}^{+}+\mathrm{HCO}_{3}^{-}
\end{aligned}
$$

These reactions are also referred to as the carbamate $\left(\mathrm{MEACOO}^{-}\right)$and bicarbonate $\left(\mathrm{HCO}_{3}^{-}\right)$ formation reactions, respectively. The solution chemistry and these reactions will be discussed in further detail in Chapter 4.2. Through the speciation model, the apparent species composition (previously denoted as $x_{i}$ for the three component mixture) is converted to the true species composition $\left(\bar{x}_{i}\right)$ as a function of temperature. The species molar volume is calculated as the average of the species molecular weights $\left(M W_{i}\right)$ weighted by the true species mole fractions. 


$$
M W_{\text {sln }}=\sum_{i=1}^{m} M W_{i} \bar{x}_{i}
$$

The density data are given in the literature in terms of the previously defined quantities $\alpha$ and $w_{M E A}^{*}$. These can be converted to the apparent species mole fractions using the following relationships derived from a material balance:

$$
\begin{gathered}
x_{M E A}=\left(1+\alpha+\left(\frac{M W_{M E A}}{M W_{H_{2} O}}\right)\left(\frac{1-w_{M E A}^{*}}{w_{M E A}^{*}}\right)\right)^{-1} \\
x_{C O_{2}}=\alpha x_{M E A} \\
x_{\mathrm{H}_{2} \mathrm{O}}=1-\left(x_{M E A}+x_{C O_{2}}\right)
\end{gathered}
$$

The new model proposed here for the solution molar volume is given by:

$$
V_{S l n}=x_{M E A} V_{M E A}+x_{H_{2} O} V_{H_{2} O}+a x_{C O_{2}}+\left(b+c x_{M E A}\right) x_{M E A} x_{H_{2} O}+\left(d+e x_{M E A}\right) x_{M E A} x_{C O_{2}}
$$

The major difference between this new model form and the original molar volume model (Eqs. 3.3-5) is that both interaction terms labeled as $V^{*}$ and $V^{* *}$ in Eq. 3.3 are assumed to be linear functions of the apparent mole fraction of MEA and the assumption that $V^{* *}=0$ is no longer considered for the new model. Only five parameters (labelled as $a-e$ ) are considered for the model regression, which are related to the non-ideality of the electrolytic solution mixture, and it is assumed that the molar volumes of $\mathrm{H}_{2} \mathrm{O}$ and MEA may be calculated with relatively high accuracy. The same calculation is used for the molar volume of MEA, with the functional form given in Eq. 3.4 and the parameter values in Table 3.2. A similar calculation is performed for the $\mathrm{H}_{2} \mathrm{O}$ molar volume $[\mathrm{mL} / \mathrm{mol}]$, with the equation given as:

$$
V_{\mathrm{H}_{2} \mathrm{O}}=\frac{M W_{\mathrm{H}_{2} \mathrm{O}}}{-\left(3.2484 \times 10^{-6}\right) T^{2}+0.00165 T+0.793}
$$

The coefficients in Eq. 3.14 are regressed from data given in Liley et al. (1997). 
Density data from three sources have been included in the regression of the parameters in Eq. 3.13 , for which ranges of predictor variable values and the number of observations are given in Table 3.3.

Table 3-3. Summary of density data applied to model regression

\begin{tabular}{ccccc}
\hline Data Source & $\begin{array}{c}\text { Number of } \\
\text { Observations }\end{array}$ & \multicolumn{2}{c}{ Predictor Variable Ranges } \\
\cline { 3 - 5 } & & Temperature (K) & $\mathrm{CO}_{2}$ Loading & Nominal MEA \\
& & & $0-0.5$ & Weight Fraction \\
\hline Amundsen et al. (2009) & 83 & $298.15-353.15$ & $0.2-0.4$ \\
Jayarathna et al. (2013) & 72 & $303.15-333.15$ & $0-0.5$ & $0.2-0.4$ \\
Han et al. (2012) & 54 & $298.15-353.15$ & $0.1-0.56$ & $0.2-0.4$ \\
\hline
\end{tabular}

The model parameters are calibrated using all data observations as described in Table 3.3, with equal weights, and the values are given in Table 3.4.

Table 3-4. Calibrated parameter values for molar volume model given in Eq. 3.13.

\begin{tabular}{cc}
\hline Parameter & Value \\
\hline a & 10.2074 \\
b & -2.2642 \\
c & 3.0059 \\
d & 207 \\
e & -563.3701 \\
\hline
\end{tabular}

The values of the AARD for the original model (Weiland et al., 1998) and the model with respect to the density calculation are given in Table 3.5. The fit of these models to the experimental data is shown in Figure 3.2. Model predictions and data are shown only for temperatures of $298.15,323.15$, and $353.15 \mathrm{~K}$ to enhance the clarity of the graphs. 
Table 3-5. Values of average absolute relative deviation for original and new density models

\begin{tabular}{ccc}
\hline Data Source & Original Model AARD (\%) & New Model AARD (\%) \\
\hline Amundsen et al. (2009) & 0.24 & 0.27 \\
Jayarathna et al. (2012) & 0.37 & 0.09 \\
Han et al. (2013) & 0.71 & 0.28 \\
All Data & 0.41 & 0.20 \\
\hline
\end{tabular}

A

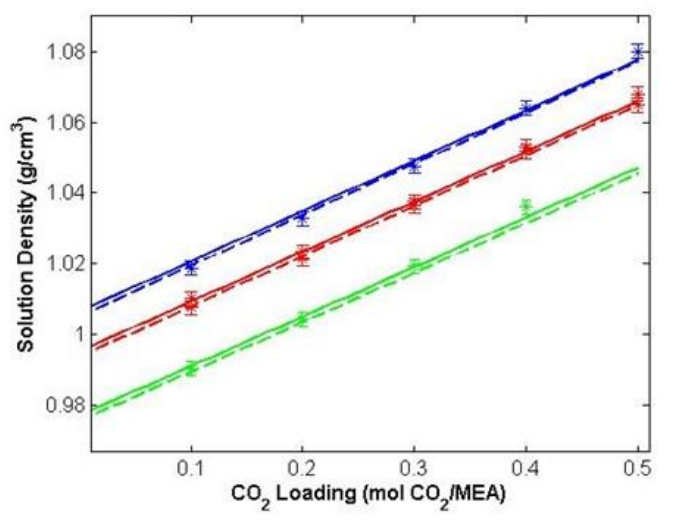

$\mathrm{C}$

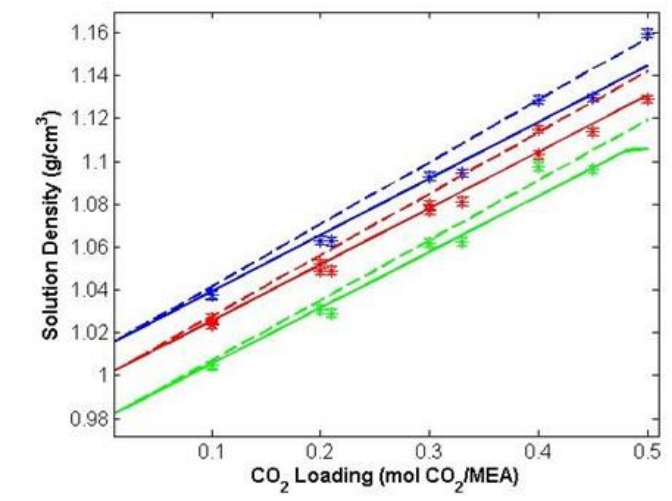

B

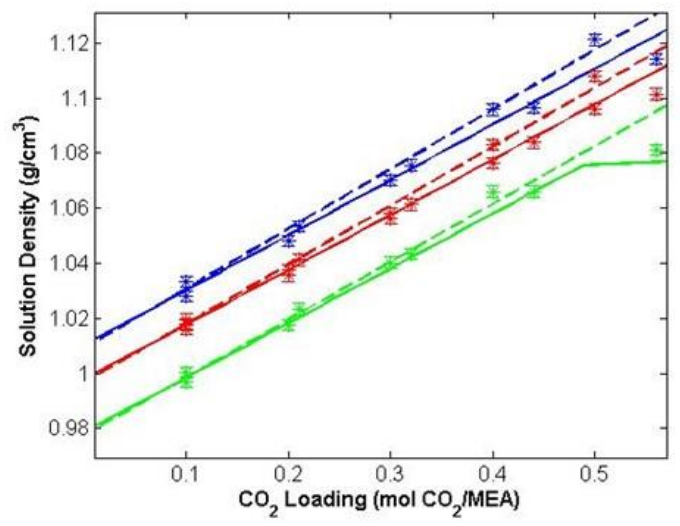

- $298.15 \mathrm{~K}$

- $323.15 \mathrm{~K}$

$353.15 \mathrm{~K}$

Figure 3-2. Comparison of models to experimental data for density of solutions with: A) 20 wt\% MEA; B) $30 \mathrm{wt} \%$ MEA; C) $40 \mathrm{wt} \%$ MEA. Stars (*) represent experimental data from sources listed in Table 3-3, dashed lines represent model from Weiland et al. (1998), and solid lines represent model developed in this work. 
For solutions of $20 \%$ MEA, the density data from all sources are in good agreement, and both the model from Weiland et al. (1998) and the model from this work fit the data satisfactorily. For solutions of 30 and $40 \%$ MEA, there is some discrepancy between the data given by different sources. The original model more accurately fits the data from Amundsen et al. (2009) than those from the other source because these data were used as validation data for this model. Since the model for this work is regressed to fit the aggregate data set, the AARD of the model compared with the Amundsen et al. (2009) data is higher than for the original model, but much lower in comparison with the data from the other sources. The new model form is evaluated with an 11fold cross validation procedure, which gives a correlation between model and data of $R^{2}=$ 0.9911.

\subsection{Surface Tension Model}

\subsubsection{Original Model}

A model for surface tension of the $\mathrm{MEA}-\mathrm{H}_{2} \mathrm{O}-\mathrm{CO}_{2}$ system $\left(\sigma_{s l n}\right)$ is presented in the work of Jayarathna et al. (2013), and given by the equation:

$$
\sigma_{s l n}=\sigma_{2}+\sum_{i=1,3}\left(1+\frac{b_{i} x_{i}}{\left(1-a_{i}\right)\left(1+\sum_{j=1,3} \frac{a_{j}}{1-a_{j}} x_{j}\right)}\right)\left(x_{i}\left(\sigma_{i}-\sigma_{2}\right)\right)
$$

The subscripts 1-3 represent $\mathrm{CO}_{2}, \mathrm{H}_{2} \mathrm{O}$, and MEA, respectively. The surface tension of component $i$ is denoted by $\sigma_{i}$. The parameters $a_{i}$ and $b_{i}$ are dependent on the mass fraction of MEA in solution. The values of the parameters are given in Table 3.6. 
Table 3-6. MEA composition dependent parameters for surface tension model

\begin{tabular}{cccc}
\hline \multicolumn{5}{c}{$w_{M E A}^{*}=0.2$} & $w_{M E A}^{*}=0.3$ & $w_{M E A}^{*}=0.4$ \\
\hline \multicolumn{4}{c}{$\mathrm{CO}_{2}(\mathrm{i}=1)$} \\
\hline$a_{i}$ & 0.3073 & 0.09409 & 0.1478 \\
$b_{i}$ & -0.8574 & -0.7392 & -0.8982 \\
\hline \multicolumn{4}{c}{ MEA $(i=3)$} \\
\hline$a_{i}$ & 1.067 & 1.114 & 1.157 \\
$b_{i}$ & 1.1701 & 0.1757 & 0.3062 \\
\hline
\end{tabular}

Since carbon dioxide does not exist as a liquid in the temperature range of interest, the surface tension associated with this component is treated as a fitting parameter in the Jayarathna et al. (2013) model. The parameter is given, as a function of temperature, as:

$$
\sigma_{\mathrm{CO}_{2}}=S 1+S 2 * T(K)
$$

where S1 and S2 are parameters that depend on the MEA content of the mixture, with values given in Table 3.7.

Table 3-7. MEA composition dependent parameters for $\mathrm{CO}_{2}$ surface tension term

\begin{tabular}{cccc}
\hline & $w_{M E A}^{*}=0.2$ & $w_{M E A}^{*}=0.3$ & $w_{M E A}^{*}=0.4$ \\
\hline S1 & 0.08286 & 0.1605 & 0.1184 \\
S2 & $4.309 \times 10^{-4}$ & $1.316 \times 10^{-4}$ & $1.954 \times 10^{-4}$ \\
\hline
\end{tabular}

The pure component surface tension values $\left(\sigma_{i}\right)$ for $\mathrm{H}_{2} \mathrm{O}$ and MEA are calculated using the model proposed by Asprion (2005), which is given by the equation:

$$
\sigma_{i}=c_{1 i}\left(1-\frac{T}{T_{c i}}\right)^{c_{2 i}+c_{3 i}\left(\frac{T}{T_{c}}\right)+c_{4 i}\left(\frac{T}{T_{c}}\right)^{2}}
$$

where $T_{C i}$ is the pure component critical temperature the species dependent parameters are denoted by $c_{1 i}-c_{4 i}$. The values of these parameters are given in Table 3.8. 
Table 3-8. Parameters for pure component surface tension $(\mathrm{N} / \mathrm{m})$ calculation

\begin{tabular}{cccccc}
\hline & $c_{1 i}$ & $c_{2 i}$ & $c_{3 i}$ & $c_{4 i}$ & $T_{C i}(K)$ \\
\hline $\mathrm{H}_{2} \mathrm{O}$ & 0.18548 & 2.717 & -3.554 & 2.047 & 647.13 \\
$\mathrm{MEA}$ & 0.09945 & 1.067 & 0 & 0 & 614.45 \\
\hline
\end{tabular}

\subsubsection{Modified Model}

Although the surface tension model of Jayarathna et al. (2013) was shown to accurately represent the experimental data, it has a major shortcoming in that it is only applicable to solutions with discrete values of MEA composition ( $w_{M E A}^{*} \in[0.2,0.7]$ in increments of 0.1 ) in its original form. Therefore, the model is updated in this work to allow representation of solution surface tension over a continuous range of composition. The range $r \in[0.2,0.4]$ is considered for this work to allow for consistency with the models previously developed for viscosity and density. For the updated surface tension model, Eq. 3.16 is replaced by:

$$
\sigma_{C O_{2}}=S 1 * r^{2}+S 2 * r+S 3+T\left(S 4 * r^{2}+S 5 * r+S 6\right)
$$

so that this fitting parameter may be represented as a continuous function of $w_{M E A}^{*}$. The parameter (S1-S6) values are regressed to give the best fit between the values of $\sigma_{\mathrm{CO}_{2}}$ calculated by Eqs. 3.16 and 3.18. In order to remove the MEA concentration-dependent parameters in Eq. 3.15 , this equation is replaced with a similar functional form:

$$
\begin{gathered}
\sigma_{S l n}=\sigma_{\mathrm{H}_{2} \mathrm{O}}+\left(\sigma_{\mathrm{CO}_{2}-} \sigma_{\mathrm{H}_{2} \mathrm{O}}\right) f\left(w_{M E A}^{*}, \alpha\right) X_{C O_{2}}+\left(\sigma_{M E A}-\sigma_{\mathrm{H}_{2} O}\right) g\left(w_{M E A}^{*}, \alpha\right) X_{M E A} \\
f\left(w_{M E A}^{*}, \alpha\right)=a+b \alpha+c \alpha^{2}+d w_{M E A}^{*}+e w_{M E A}^{*}{ }^{2} \\
g\left(w_{M E A}^{*}, \alpha\right)=f+g \alpha+h \alpha^{2}+i w_{M E A}^{*}+j w_{M E A}^{*}{ }^{2}
\end{gathered}
$$

Model parameters (a-j) are regressed with experimental data from Jayarathna et al. (2013). The regressed values of the parameters for Eq. 3.18 and 3.20-21 are given in Tables 3.9 and 3.10, respectively. The fit of the new and original surface tension models is compared in Figure 3.3. 
Table 3-9. Parameters for updated $\mathrm{CO}_{2}$ surface tension term

\begin{tabular}{lc}
\hline S1 & -5.987 \\
S2 & 3.7699 \\
S3 & -0.43164 \\
S4 & 0.018155 \\
S5 & -0.01207 \\
S6 & 0.002119 \\
\hline
\end{tabular}

Table 3-10. Regressed parameter values for new surface tension model

\begin{tabular}{cc|cc}
\hline Parameter & Value & Parameter & Value \\
\hline a & 2.4558 & $\mathrm{f}$ & 2.3122 \\
$\mathrm{~b}$ & -1.5311 & $\mathrm{~g}$ & 4.5608 \\
$\mathrm{c}$ & 3.4994 & $\mathrm{~h}$ & -2.3924 \\
$\mathrm{~d}$ & -5.6398 & $\mathrm{i}$ & 5.3324 \\
$\mathrm{e}$ & 10.2109 & $\mathrm{j}$ & -12.0494 \\
\hline
\end{tabular}


A

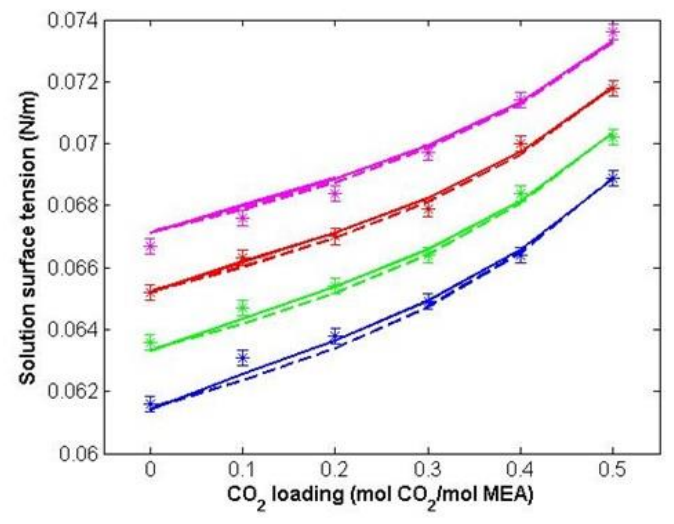

$\mathrm{B}$

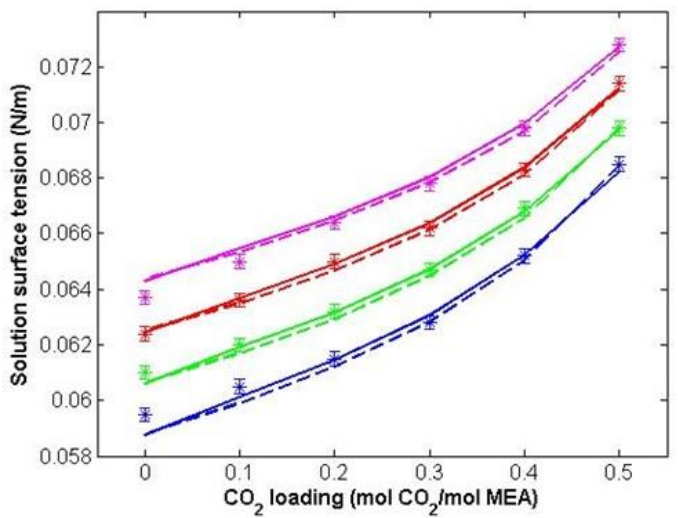

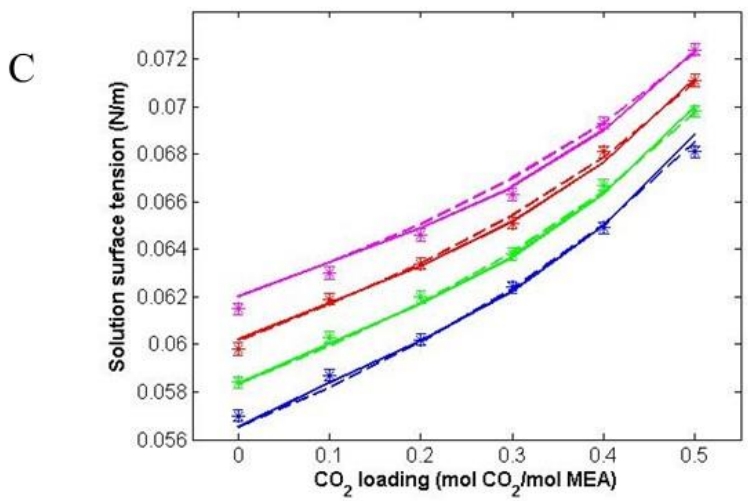

$-303.15 \mathrm{~K}$
$-313.15 \mathrm{~K}$
$-323.15 \mathrm{~K}$
$-\quad 333.15 \mathrm{~K}$

Figure 3-3. Comparison of models to experimental data for solutions of: A) $20 \mathrm{wt} \% \mathrm{MEA}$; B) $30 \mathrm{wt} \%$ MEA; C) $40 \mathrm{wt} \%$ MEA. Stars (*) represent experimental data from Jayarathna et al. (2013), dashed lines represent original model, and solid lines represent new model.

The fits of the new and original models to the experimental data are similar, but, as mentioned previously, the model by Jayarathna et al. (2013) is valid only for discrete values of $w_{M E A}^{*}$. The new model may be used to calculate solution surface tension over relevant ranges of composition as well as temperature, making applicable for implementation in a process model. The values of AARD are $0.38 \%$ and $0.35 \%$ for the original and new models, respectively. The new model is evaluated with a 9-fold cross validation procedure, giving a correlation of $R^{2}=0.9914$.

\subsection{Conclusions}

In this work, a generalized approach to developing deterministic models for physical properties has been presented, which involves determining a reasonable model form and fitting experimental data from the open literature. The viscosity, molar volume, and surface tension 
models of the $\mathrm{CO}_{2}$-loaded aqueous MEA system have been developed to be valid for a wide range of operating conditions. The optimal baseline model parameters have been determined for each correlation by minimizing SSE and the model forms have been tested using cross validation. This procedure has been implemented for these simple standalone models, and it will be extended in the following chapter for the thermodynamic framework of the $\mathrm{MEA}-\mathrm{CO}_{2}-\mathrm{H}_{2} \mathrm{O}$ system, in which multiple types of data will be implemented into a single model regression. The standalone models developed in this chapter will be revisited in Chapter 5, in which the parametric uncertainty is quantified. 


\section{Chapter 4 : MEA Thermodynamic Framework}

This section describes the development of a thermodynamic framework using the e-NRTL activity coefficient model as a starting point. Example of two thermodynamic models that use this same activity coefficient model are the model available as part of the Aspen Plus example library for the MEA system (Aspen Plus documentation, 2013a) and the "Phoenix model" developed by Plaza (2012). Both models have been validated with an extensive set of thermodynamic data that span the temperature and composition ranges applicable to absorber and stripper columns in a solvent-based $\mathrm{CO}_{2}$ capture process. Accordingly, these two models will be referred to throughout this work as a basis of comparison for the new model developed herein. The Aspen Plus library model has been developed considering binary and ternary VLE, heat capacity, and heat of absorption data. The Plaza model, however, does not include heat of absorption data or binary system VLE data in its regression.

\subsection{Background for ELECNRTL Method in Aspen Plus}

In this work, the thermodynamic model is developed in Aspen Plus ${ }^{\circledR}$ and parameters are calibrated to fit relevant experimental data, and the model equations are described here (Aspen Plus documentation, 2013b).

The condition for vapor-liquid equilibrium for a mixture is as follows; for each component $i$ in equilibrium:

$$
\hat{f}_{i}^{V}=\hat{f}_{i}^{L}
$$

where $\hat{f}_{i}^{V}$ and $\hat{f}_{i}^{L}$ are the vapor and liquid phase componenet fugacities. Considering that an activity coefficient model is used for liquid phase nonidealities, Eq. 4.1 can be written as:

$$
\hat{\varphi}_{i} y_{i} P=\gamma_{i} x_{i} f_{i}^{o}
$$

where $x_{i}$ and $y_{i}$ are the liquid and vapor phase mole fractions, $P$ is the system pressure, $\hat{\varphi}_{i}$ is the vapor phase fugacity coefficient, $\gamma_{i}$ is the symmetric activity coefficient, and $f_{i}^{o}$ is the liquid phase reference fugacity. Note that $x_{i}$ refers to true, rather than apparent, species mole fraction in all thermodynamic model equations. For solvents, the liquid phase reference fugacity is calculated as:

$$
f_{i}^{o}=\varphi_{i}^{s a t} P_{i}^{s a t} \exp \left(\frac{1}{R T} \int_{P_{i}^{s a t}}^{P} V_{i}^{L} d P\right)
$$


where $P_{i}^{\text {sat }}$ and $V_{i}^{L}$ are the vapor pressure and molar volume of species $i$, and $\varphi_{i}^{\text {sat }}$ is the pure component fugacity coefficient evaluated at the system temperature and vapor pressure. For components in which Henry's law is applied, the liquid phase reference fugacity is calculated as:

$$
f_{i}^{o}=\frac{k_{H i}}{\lim _{x_{i} \rightarrow 0} \gamma_{i}}=\frac{k_{H i}}{\gamma_{i}^{\infty}}
$$

where $k_{H i}$ is the Henry's constant, a function of temperature. The symmetric activity coefficient of species $i$ is calculated as:

$$
\ln \left(\gamma_{i}\right)=\left.\frac{1}{R T} \frac{\partial\left(n G^{e x}\right)}{\partial n_{i}}\right|_{T, P, n_{j \neq i}}
$$

where $n$ and $n_{i}$ are the total number of moles and the number of moles of component $\mathrm{i}$ and $G^{e x}$ is excess Gibbs free energy of solution. The infinite dilution activity coefficient $\left(\gamma_{i}^{\infty}\right)$ is defined as in the denominator of Eq. 4.4, and the asymmetric activity coefficient is defined as:

$$
\gamma_{i}^{*}=\frac{\gamma_{i}}{\gamma_{i}^{\infty}}
$$

Furthermore, the excess enthalpy $\left(H^{e x}\right)$ is related to the activity coefficient by:

$$
H^{e x}=-\left.R T^{2} \sum_{i} x_{i}\left(\frac{\partial \ln \gamma_{i}}{\partial T}\right)\right|_{P, x}
$$

The heat capacity of a liquid mixture $\left(C_{p, m}{ }^{l}\right)$, can be calculated as the temperature derivative of the liquid mixture enthalpy $\left(H_{m}^{l}\right)$, given the relationship:

$$
H_{m}{ }^{l}(T+\Delta T)-H_{m}{ }^{l}(T)=\int_{T}^{T+\Delta T} C_{p, m}{ }^{l} d T
$$

Heat of absorption can be calculated by an energy balance on a mixing process:

$$
\Delta H_{a b s}=\frac{m_{\text {final }} H_{\text {final }}-m_{\text {initial }} H_{\text {initial }}-m_{\mathrm{CO}_{2}} \mathrm{H}_{\mathrm{CO}_{2}}}{n_{\mathrm{CO}_{2}}}
$$


where $m_{\text {initial }}$ and $m_{\text {final }}$ are the initial and final mass units of solvent to which $m_{\mathrm{CO}_{2}}$ mass units $\left(n_{\mathrm{CO}_{2}}\right.$ molar units) of $\mathrm{CO}_{2}$ are added, and the mass enthalpy terms are defined analogously. Eq. 9 is valid for two distinct definitions of heat of absorption, the differential and integral heat of absorption (Zhang et al., 2011). The differential heat of absorption refers to the heat effect associated with adding an incremental amount of $\mathrm{CO}_{2}$ to a solution of specific $\mathrm{CO}_{2}$ loading. In contrast, the integral heat of absorption is the heat effect due to adding the total amount of $\mathrm{CO}_{2}$ to an unloaded solution required to increase the solution loading to a specific value. For a nonideal system, the excess enthalpy term is essential in the enthalpy model on which the heat capacity and heat of absorption are dependent. Moreover, for a reactive system, the reaction equilibrium constant $\left(K_{e q}\right)$ is related to the Gibbs free energy of reaction $\left(\Delta G_{r x n}\right)$ by:

$$
\Delta G_{r x n}=-R T \ln K_{e q}
$$

where $\Delta G_{r x n}$ is also dependent on the excess Gibbs energy for a non-ideal system. Since the VLE, heat capacity, heat of absorption, and reaction equilibrium for this system are all related to the activity coefficient model, it is clear that a consistent thermodynamic framework that incorporates all of these properties is required.

In this work, the thermodynamic framework of the MEA- $\mathrm{H}_{2} \mathrm{O}-\mathrm{CO}_{2}$ system is modeled by the ELECNRTL property method in Aspen Plus ${ }^{\circledR}$ (Aspen Plus documentation, 2013b), which uses the Redlich-Kwong equation of state to calculate the vapor phase fugacity coefficients and the electrolyte Non-Random Two-Liquid (e-NRTL) model (Chen et al., 1982) to calculate the excess Gibbs free energy. For the e-NRTL model, the excess Gibbs energy is given as a sum of three components:

$$
G^{e x}=G_{P D H}^{e x}+G_{B o r n}^{e x}+G_{L C}^{e x}
$$

The first two terms, the Pitzer-Debye-Hückel model and the Born equation are used to represent the contribution of the long range ion-ion interactions. The third term, which includes the model parameters that may be adjusted to fit experimental data, represents the contribution of local interactions. This term is given by: 


$$
\begin{gathered}
G_{L C}^{e x}=\sum_{m} X_{m} \frac{\sum_{j} X_{j} G_{j m} \tau_{j m}}{\sum_{k} X_{k} G_{k m}}+\sum_{c} X_{c} \sum_{a^{\prime}}\left(\frac{X_{a^{\prime}}}{\sum_{a^{\prime \prime}} X_{a^{\prime \prime}}}\right) \frac{\sum_{j} X_{j} G_{j c, a^{\prime} c} \tau_{j c, a^{\prime} c}}{\sum_{k} X_{k} G_{k c, a^{\prime} c}} \\
+\sum_{a} X_{a} \sum_{c^{\prime}}\left(\frac{X_{c^{\prime}}}{\sum_{c^{\prime \prime}} X_{c^{\prime \prime}}}\right) \frac{\sum_{j} X_{j} G_{j a, c^{\prime} a} \tau_{j a, c^{\prime} a}}{\sum_{k} X_{k} G_{k a, c^{\prime} a}}
\end{gathered}
$$

Here, molecules are represented by the subscript m, cations by $c, c^{\prime}$, and $c^{\prime \prime}$, and anions by $a, a^{\prime}$, and $a$ '”. The subscripts $j$ and $k$ represent any species. The terms $G$ are by defined by the convention:

$$
\begin{gathered}
G_{i m}=\exp \left(-\alpha_{i m} \tau_{i m}\right) \\
G_{c a, m}=\exp \left(-\alpha_{c a, m} \tau_{c a, m}\right)
\end{gathered}
$$

with other interaction terms defined analogously. The terms $X$ are defined by:

$$
X_{j}=x_{j} L_{j}
$$

where $x_{j}$ is the liquid phase mole fraction and $L_{j}$ is unity for molecular species and the charge number for ionic species. The terms $\alpha$ and $\tau$ are nonrandomness factors and energy parameters, respectively. Molecule-molecule binary parameters and electrolyte-molecule pair parameters are defined, respectively, as:

$$
\begin{gathered}
\tau_{m m^{\prime}}=A_{m m^{\prime}}+\frac{B_{m m^{\prime}}}{T}+E_{m m^{\prime}} \ln (T) \\
\tau_{c a, m}=C_{c a, m}+\frac{D_{c a, m}}{T}+E_{c a, m}\left(\frac{T^{r e f}-T}{T}+\ln \left(\frac{T}{T^{r e f}}\right)\right)
\end{gathered}
$$

Molecule-electrolyte and electrolyte-electrolyte pair parameters are defined analogously to the electrolyte-molecule pair parameters. In Eq. 4.17, the reference temperature is $298.15 \mathrm{~K}$ and all terms aside from temperature are fitting parameters.

In this model, the liquid enthalpy of a mixture is calculated by:

$$
H_{m}{ }^{l}(T)=\sum_{i} x_{i} H_{i}+\sum_{k} x_{k} H_{k}^{\infty}+H^{e x}
$$

where $i$ denotes molecular species and $k$ denotes electrolyte species. The enthalpy of solvents is defined as: 


$$
H_{i}(T)=\Delta H_{f}^{i g}\left(T^{r e f}\right)+\int_{T^{r e f}}^{T} C_{p, i}^{i g} d T+\Delta H_{i}^{d e p}(T, p)-\Delta H_{i}^{v a p}(T)
$$

and the enthalpy of molecular solutes, or Henry's components, is:

$$
H_{i}(T)=H_{i}^{i g}(T)-R T^{2}\left(\frac{\partial \ln k_{H i}}{\partial T}\right)
$$

The enthalpy of ionic species is:

$$
H_{k}^{\infty}(T)=\Delta H_{f, k}^{\infty}\left(T^{r e f}\right)+\int_{T^{r e f}}^{T} C_{p, k}^{\infty} d T
$$

Adjustable parameters in these equations include the ideal gas enthalpy of formation of molecular species $\left(\Delta H_{f}^{i g}\left(T^{r e f}\right)\right)$ at reference temperature $298.15 \mathrm{~K}$ and the aqueous infinite dilution heat of formation of ionic species $\left(\Delta H_{f, k}^{\infty}\left(T^{r e f}\right)\right)$. In Eq. 4.19 , the term $\Delta H_{i}^{d e p}(T, p)$ is the vapor enthalpy of departure of solvent and $\Delta H_{i}^{v a p}(T)$ is the heat of vaporization of solvent. In Eq. 4.20, $k_{H i}$ is the Henry's constant, defined, for a mixed solvent, as:

$$
\begin{gathered}
\ln \left(\frac{k_{H i}}{\gamma_{i}^{\infty}}\right)=\sum_{A} \frac{x_{A}\left(V_{c A}\right)^{2 / 3}}{\sum_{B} x_{B}\left(V_{c B}\right)^{2 / 3}} \ln \left(k_{H i A} / \gamma_{i A}^{\infty}\right) \\
k_{H i A}(T, P)=k_{H i A}\left(T, P_{A}^{*}\right) \exp \left(\frac{1}{R T} \int_{P_{A}^{s a t}}^{P} V_{i A}^{\infty} d p\right) \\
\ln \left\{k_{H i A}\left(T, P_{A}^{s a t}\right)\right\}=a_{i A}+\frac{b_{i A}}{T}+c_{i A} \ln (T)+d_{i A} T+\frac{e_{i A}}{T^{2}}
\end{gathered}
$$

In Eq. 4.23, $k_{H i}$ and $k_{H i A}$ refer to the Henry's constant in the mixture and in pure solvent $A$, respectively, and infinite dilution activity coefficients $\gamma_{i}^{\infty}$ and $\gamma_{i A}^{\infty}$ are defined analogously. The mole fraction and critical volume of $A$ (or $B$ ) are $x_{A}\left(x_{B}\right)$ and $V_{C A}\left(V_{c B}\right)$. In Eq. 4.23, $V_{i A}^{\infty}$ is the partial molar volume of species $i$ at infinite dilution in pure solvent $A$, as calculated by the Brelvi-O'Connell model (Aspen Plus documentation, 2013c). The correlation in Eq. 4.24 includes the adjustable parameters in the Henry's constant model ( $a_{i A}, b_{i A}$, etc.) for a binary species pair. Additional adjustable parameters are included in the ideal gas $\left(C_{p, i}^{i g}\right)$ and aqueous infinite dilution $\left(C_{p, k}^{\infty}\right)$ heat capacity equations which are given by:

$$
C_{p, i}^{i g}=C_{1 i}+C_{2 i} T+C_{3 i} T^{2}+C_{4 i} T^{3}+C_{5 i} T^{4}+C_{6 i} T^{5}
$$




$$
C_{p, k}^{\infty}=C_{1 k}+C_{2 k} T+C_{3 k} T^{2}+\frac{C_{4 k}}{T}+\frac{C_{5 k}}{T^{2}}+\frac{C_{6 k}}{\sqrt{T}}
$$

\subsection{Reaction Kinetics}

As previously discussed with respect to the molecular weight calculation for the density model, the MEA- $\mathrm{CO}_{2}-\mathrm{H}_{2} \mathrm{O}$ system is modeled as a reactive system with the equilibrium reactions given in Eqs. 3.7-8. Although other ionic species (e.g. $\mathrm{H}^{+}, \mathrm{OH}^{-}$, and $\mathrm{CO}_{3}^{2-}$ ) appear in solution, their concentrations are shown to be negligible at the process conditions of interest (Hilliard, 2008). These two reactions are also assumed to fully represent the chemistry of the $\mathrm{CO}_{2}$-loaded aqueous MEA system in another work (Han, Zhou, Wu, Tempel, \& Chang, 2011), in which molecular dynamics is employed to study the mechanisms of these reactions. As discussed earlier, typical power law kinetics often used in the models presented in the literature do not correctly capture the complex temperature dependence of chemical equilibrium for the electrolyte systems. Therefore, the reaction kinetics are modified to the form:

$$
\begin{aligned}
& r_{1}=A_{1}^{f} \exp \left(-\frac{E_{1}^{f}}{R}\left(\frac{1}{T}-\frac{1}{298.15}\right)\right) a_{M E A}^{2} a_{C_{2}}\left(1-\frac{a_{M E A^{+}} a_{M E A C O O}}{K_{1} a_{M E A}^{2} a_{C O_{2}}}\right) \\
& r_{2}=A_{2}^{f} \exp \left(-\frac{E_{2}^{f}}{R}\left(\frac{1}{T}-\frac{1}{298.15}\right)\right) a_{M E A} a_{C O_{2}}\left(1-\frac{a_{M E A^{+}} a_{\mathrm{HCO}_{3}^{-}}}{K_{2} a_{M E A} a_{C O_{2}} a_{H_{2} O}}\right)
\end{aligned}
$$

The form given in Eqs. 4.27-28 is similar to that of the work of Mathias and Gilmartin (2014) for an AMP (2-Amino-2-methyl-1-propanol) $-\mathrm{H}_{2} \mathrm{O}-\mathrm{CO}_{2}$ system. The equilibrium constants of the reactions are calculated as:

$$
\begin{gathered}
K_{1}=\left.\frac{\gamma_{M E A^{+}} x_{M E A^{+}} \gamma_{M E A C O O}-x_{M E A C O O}}{\left(\gamma_{M E A}^{*} x_{M E A}\right)^{2} \gamma_{C O_{2}}^{*} x_{C O_{2}}}\right|_{e q} \\
K_{2}=\left.\frac{\gamma_{M E A^{+}} x_{M E A^{+}} \gamma_{\mathrm{HCO}_{3}^{-}} x_{\mathrm{HCO}_{3}^{-}}}{\gamma_{M E A}^{*} x_{M E A} \gamma_{C O_{2}}^{*} x_{\mathrm{CO}_{2}} \gamma_{\mathrm{H}_{2} \mathrm{O}} x_{\mathrm{H}_{2} \mathrm{O}}}\right|_{e q}
\end{gathered}
$$


where $x_{i}$ denotes the liquid phase mole fraction of a given species and $\gamma_{i}$ and $\gamma_{i}^{*}$ represent symmetrical and unsymmetrical activity coefficients, noting that unsymmetrical activity coefficients are used for $\mathrm{CO}_{2}$ and MEA, which are modeled as Henry's coefficients in this work. In this work, the pre-exponential factor and activation energy for the forward reactions are taken from the work of Plaza (2012), and shown in Table 4.1. Since this reaction kinetics model is not in one of the standard forms used in Aspen Plus ${ }^{\circledR}$, it is incorporated into the simulation through a FORTRAN user model. The user model is contained in Appendix C.4.

Table 4-1. Reaction kinetics parameters taken from Plaza (2012)

\begin{tabular}{cc}
\hline Pre-Exponential Factor $\left(\mathrm{kmol} / \mathrm{m}^{3}-\mathrm{s}\right)$ & \\
\hline$A_{1}^{f}$ & $8.56 \times 10^{10}$ \\
$A_{2}^{f}$ & $2.30 \times 10^{4}$ \\
\hline$E_{1}^{f}$ & \\
\hline$E_{2}^{f}$ & $3.96 \times 10^{3}$ \\
\hline Activation Energy $(\mathrm{J} / \mathrm{mol})$ & $4.90 \times 10^{4}$ \\
\hline
\end{tabular}

\subsection{Parameter Regression}

\subsubsection{Methodology}

The Aspen Plus ${ }^{\circledR}$ data regression system is used to calibrate the parameter values to fit the experimental VLE data for the binary MEA- $\mathrm{H}_{2} \mathrm{O}$ system, the ternary MEA- $\mathrm{H}_{2} \mathrm{O}-\mathrm{CO}_{2}$ system, differential heat of absorption, and heat capacity data for the ternary system. Since heat of absorption is not directly incorporated into Aspen Plus ${ }^{\circledR}$ as a physical property that can be included in the data regression, it has been included through a FORTRAN user model, in which it is calculated as in Eq. 4.9. The objective function to be minimized is the sum of squared error weighed by the reciprocal of the variance of the measurements.

A separate regression is performed to calibrate values for the heat capacity polynomials, including the ideal gas heat capacity of MEA (Eq. 25) and the aqueous infinite dilution heat capacity of $\mathrm{MEA}^{+}$and $\mathrm{MEACOO}^{-}$(Eq. 26). Each of the three polynomials is truncated to two terms. This regression only includes the heat capacity data, because it has been determined that the model calculations of VLE and heat of absorption are not sensitive to these parameters, so it is not necessary to include them in the regression with the full set of data and parameters. Thus, 
these parameters are fixed at the values determined in this regression in the subsequent simultaneous regression.

The simultaneous data regression incorporates data for $\operatorname{VLE}\left(\mathrm{CO}_{2}\right.$ and MEA partial pressure), heat capacity, and heat of absorption data for the ternary $\mathrm{MEA}-\mathrm{H}_{2} \mathrm{O}-\mathrm{CO}_{2}$ system as well as additional VLE data for the binary $\mathrm{MEA}-\mathrm{H}_{2} \mathrm{O}$ system. Due to the large number of parameters available for inclusion in the e-NRTL thermodynamic model, it is essential to include a parameter selection step in the model development methodology. Even with the simplified speciation model, given in Eqs. 3.7-8, there are approximately 60 parameters available for inclusion in the Gibbs free energy model. Parameter selection is necessary not only to avoid over-parameterization in the deterministic model, but to improve the computational feasibility of UQ. For the thermodynamic framework outlined previously, the Akaike Information Criterion (AIC) (Akaike, 1974) is used to determine the form of the final deterministic model. In this analysis, it is desired to minimize the AIC, so as to determine an optimal model to fit data without over-parameterization. The AIC may be expressed as:

$$
A I C=N \ln \left(\frac{S S E}{N}\right)+2 k
$$

where SSE refers to the sum of square error between data values and model fit, $k$ the number of parameters included in the model, and $N$ the number of data used for calibrating the model parameters.

The first regression is performed for a baseline model in which the parameters included are the reference temperature $\left(25^{\circ} \mathrm{C}\right)$ Gibbs free energy and enthalpy of formation of ionic species (represented as DGAQFM and DHAQFM in Aspen Plus) for species MEA ${ }^{+}$and MEACOO ${ }^{-}$and the parameters in the model for Henry's constant for the MEA- $\mathrm{H}_{2} \mathrm{O}$ pair. The Henry's constant parameters for the MEA- $\mathrm{H}_{2} \mathrm{O}$ binary pair are necessary because MEA is assumed to follow Henry's law in this work, similar to the Plaza (2012) model, and the solubility of MEA in $\mathrm{H}_{2} \mathrm{O}$ has not been well characterized in previous works. Therefore, the Henry parameters for this binary pair are treated as fitting parameters for the model. All NRTL molecule-molecule binary and electrolyte-molecule pair parameters are taken to be zero for this baseline model. Values for the parameters not mentioned here (e.g. pure component physical properties for MEA and $\mathrm{H}_{2} \mathrm{O}$ ) are taken from the library model available in Aspen Plus (Aspen Plus documentation, 2013a), 
and are not regressed in this work. In subsequent regressions, NRTL binary and pair parameters are added incrementally until the AIC is minimized.

\subsubsection{Results}

The values of the heat capacity polynomial parameters are presented in Table 4.2. Note that these parameters are fixed at these values for the simultaneous regression in which all data are included.

Table 4-2. Regressed values of heat capacity (J/kmol-K) polynomial parameters

\begin{tabular}{cc}
\hline Parameter & Value \\
\hline CPIG/1 (MEA) & $5.08 \times 10^{4}$ \\
CPIG/2 (MEA) & 336 \\
CPAQ/1 (MEACOO-) & -0.598 \\
CPAQ/2 (MEACOO-) & 42.3 \\
CPAQ/1 (MEA+) & $7.39 \times 10^{5}$ \\
CPAQ/2 (MEA+) & $-1.63 \times 10^{3}$ \\
\hline
\end{tabular}

In the parameter selection methodology, the binary and pair NRTL parameters are incrementally included in the model regression to determine the optimal model based on the AIC values. The results of this study are shown in Table 4.3. For each parameter number given in the table below, combinations of parameters have been considered and the lowest AIC for that specific number of parameters has been reported.

Table 4-3. Model AIC for varying number of NRTL parameters

\begin{tabular}{cc}
\hline Number of Parameters & AIC Value $\times 10^{-3}$ \\
\hline Baseline & 2.10 \\
1 & 1.91 \\
2 & 1.19 \\
3 & 1.16 \\
4 & 1.12 \\
5 & 1.13 \\
\hline
\end{tabular}

Since it has been determined that no five parameter model has an AIC lower than the four parameter model with the lowest AIC, the four parameter model is taken as the final deterministic model. The regression results are presented in the form of parameter values and 
standard deviations, shown in Table 4.4, and a matrix of correlation coefficients, shown in Table 4.5.

Table 4-4. Values of parameters included in thermodynamic framework

\begin{tabular}{|c|c|c|c|}
\hline $\begin{array}{c}\text { Parameter } \\
\text { No. }\end{array}$ & Parameter Name & Value & Standard Deviation \\
\hline \multicolumn{4}{|c|}{ Electrolyte Species Formation Parameters (MJ/kmol) } \\
\hline 1 & DGAQFM (MEA+) & -190 & 0.237 \\
\hline 2 & DGAQFM (MEACOO-) & -492 & 0.252 \\
\hline 3 & DHAQFM (MEA+) & -330 & 1.01 \\
\hline 4 & DHAQFM (MEACOO-) & -691 & 1.15 \\
\hline \multicolumn{4}{|c|}{ Henry’s Constant Parameters (Pa) } \\
\hline 5 & HENRY/1 (MEA- $\left.{ }_{2} \mathrm{O}\right)$ & 28.6 & 0.183 \\
\hline 6 & HENRY/2 (MEA- $\left.{ }_{2} \mathrm{O}\right)$ & -7610 & 65.0 \\
\hline \multicolumn{4}{|c|}{ NRTL Molecule-Molecule Binary Parameters } \\
\hline 7 & NRTL/1 (MEA-H $\left.{ }_{2} \mathrm{O}\right)$ & 3.25 & 0.196 \\
\hline 8 & NRTL/1 $\left(\mathrm{H}_{2} \mathrm{O}-\mathrm{MEA}\right)$ & 4.34 & 0.481 \\
\hline 9 & NRTL/1 $\left(\mathrm{CO}_{2}-\mathrm{H}_{2} \mathrm{O}\right)$ & 69.4 & 4.18 \\
\hline 10 & NRTL/2 $\left(\mathrm{H}_{2} \mathrm{O}-\mathrm{MEA}\right)$ & -2200 & 185 \\
\hline
\end{tabular}


Table 4-5. Correlation coefficients of parameters in thermodynamic framework regression

\begin{tabular}{c|ccccccccc} 
& 1 & 2 & 3 & 4 & 5 & 6 & 7 & 8 & \\
\hline 1 & 1 & & & & & & & & \\
2 & -0.587 & 1 & & & & & & \\
3 & 0.388 & 0.0984 & 1 & & & & & \\
4 & 0.153 & 0.313 & -0.355 & 1 & & & \\
5 & -0.335 & -0.396 & -0.540 & -0.415 & 1 & & & \\
6 & 0.344 & 0.398 & 0.540 & 0.418 & -0.998 & 1 & & 1 \\
7 & -0.0401 & 0.337 & 0.276 & -0.0381 & -0.340 & 0.327 & 1 & \\
8 & -0.133 & -0.245 & -0.242 & -0.185 & 0.482 & -0.488 & -0.313 & 0.00139 & 1 \\
9 & -0.147 & 0.0988 & -0.210 & 0.123 & $-2.29 \mathrm{e}-4$ & $-5.16 \mathrm{e}-4$ & 0.0160 & $-5.11 \mathrm{e}-5$ & 1
\end{tabular}


Table 4.4 shows the parameters included in the final model, and their values that were determined from simultaneous regression. The electrolyte species formation parameters and Henry constant parameters are included as part of the baseline model and in all subsequent regressions. The set of four NRTL binary pair parameters included has been determined from the AIC analysis. Although electrolyte-molecule pair parameters have been considered in this analysis, none are included in the final model. This results in a substantial simplification to the model form, noting that of the three terms in the local contribution to the Gibbs free energy (Eq. 4.12), only the first remains in the final version of this model. By including this parameter selection methodology, it is shown that a reduced version of the e-NRTL model, containing a relatively small number of parameters, can adequately represent the thermodynamics of this system. By comparison, the model developed by Plaza (2012), which will be compared with this model later in this work, includes 41 regressed parameters.

The most widely available type of thermodynamic property data available in the open literature is ternary system VLE data, for which $\mathrm{CO}_{2}$ and MEA partial pressure values are available for variable temperature, $\mathrm{CO}_{2}$ loading, and nominal MEA weight fraction. A summary of the $\mathrm{CO}_{2}$ partial pressure data considered in the model regression is given in Table 4.6. MEA partial pressure from Hilliard (2008) is also used, with the same number of data and variable ranges as given for the $\mathrm{CO}_{2}$ partial pressure data from this source. 
Table 4-6. $\mathrm{CO}_{2}$ partial pressure data used in thermodynamic framework regression

\begin{tabular}{cccccc}
\hline Data Source & $\begin{array}{c}\text { Number of } \\
\text { Data }\end{array}$ & Temperature $\left({ }^{\circ} \mathrm{C}\right)$ & $\begin{array}{c}\text { Loading } \\
(\mathrm{mol} \mathrm{CO} / \mathrm{MEA})\end{array}$ & $\begin{array}{c}\text { MEA weight } \\
\text { percent }\end{array}$ & $\begin{array}{c}\mathrm{CO}_{2} \text { partial pressure } \\
(\mathrm{kPa})\end{array}$ \\
\hline Aronu et al. (2011) & 138 & $40-80$ & $0.017-0.565$ & $15-45$ & $0.0007-19$ \\
Hilliard (2008) & 55 & $40-60$ & $0.114-0.591$ & $17-40$ & $0.005-50$ \\
Jou et al. (1995) & 46 & $25-120$ & $0.003-0.589$ & 30 & $0.0015-822$ \\
Dugas (2009) & 50 & $40-100$ & $0.231-0.500$ & $30-45$ & $0.01-29$ \\
Lee et al. (1976) & 155 & $25-120$ & $0.065-0.600$ & $6.5-32$ & $0.1-1000$ \\
Xu (2011) & 36 & $100-130$ & $0.313-0.520$ & 30 & $12-1000$ \\
Ma'mun et al. (2005) & 19 & 120 & $0.155-0.418$ & 30 & $7-192$ \\
\hline
\end{tabular}


The ranges of the input variables completely span, and in some cases exceed, the values of interest for industrial $\mathrm{CO}_{2}$ capture applications. Figure 4.1 shows a comparison of the ternary system $\mathrm{CO}_{2}$ partial pressure data to the models from Plaza (2012), the library of Aspen Plus (Aspen Plus documentation, 2013a), and this work (denoted as CCSI model).
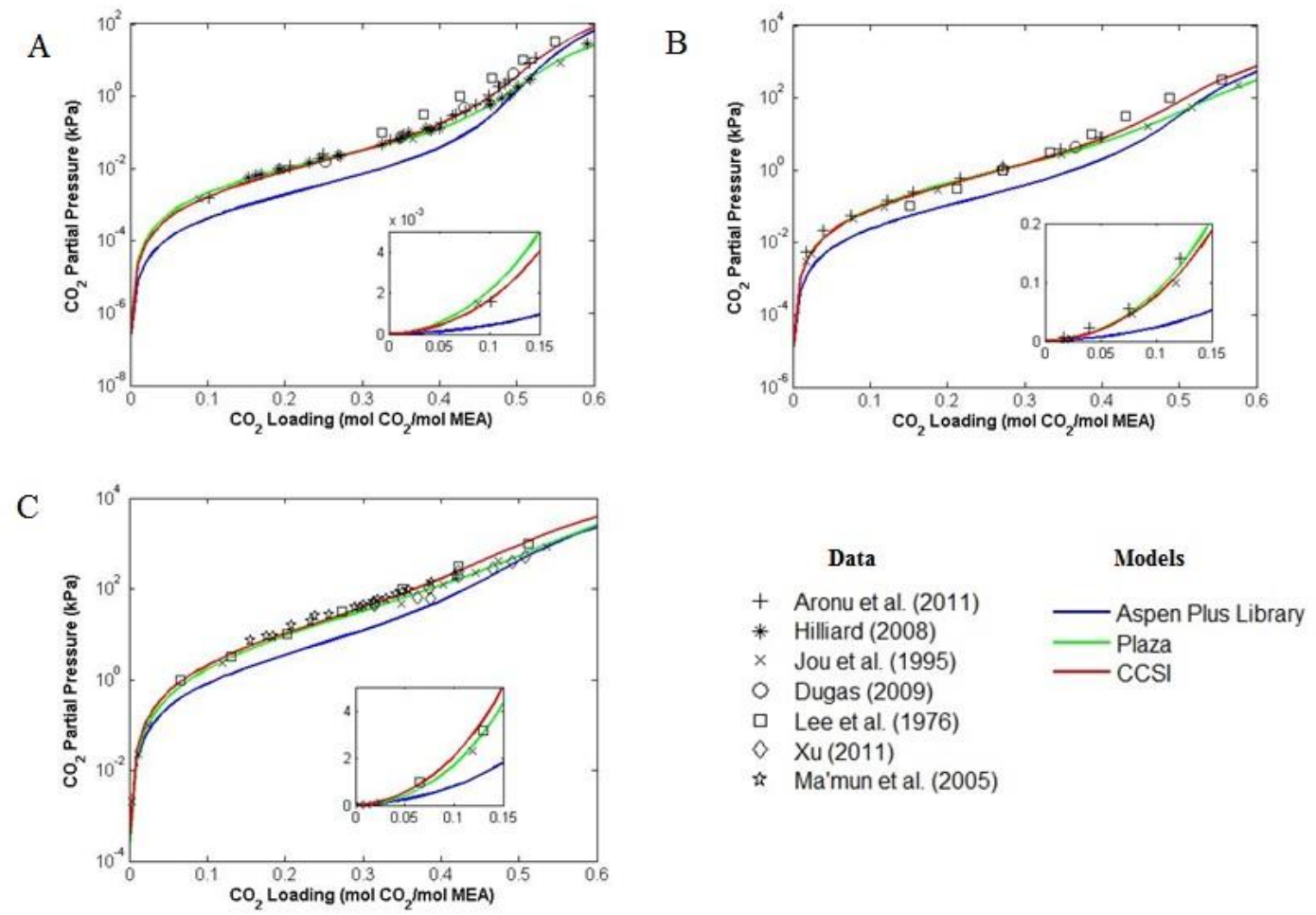

Figure 4-1. Comparison of $\mathrm{CO}_{2}$ partial pressure data from various sources for solutions of 30 wt \% MEA and temperatures of $(\mathrm{A}) 40^{\circ} \mathrm{C},(\mathrm{B}) 80^{\circ} \mathrm{C}$, and $(\mathrm{C}) 120^{\circ} \mathrm{C}$ as a function of $\mathrm{CO}_{2}$ loading to new and existing models.

As shown in Figure 4.1, the fit of the CCSI model to these experimental data is adequate, and comparable to that of the Plaza model, despite the fact that a much smaller number of parameters is used. The fit is shown for the conditions for which the majority of the experimental are given, namely $30 \mathrm{wt} \%$ MEA and temperature values of 40,80 , and $120^{\circ} \mathrm{C}$. Inset plots are included, on a linear scale, for each temperature to clarify the fit of each model to the data at low values of loading. Temperatures of $40-80^{\circ} \mathrm{C}$ correspond to typical absorber operation, for which a large temperature range should be considered due to temperature bulges occurring as a result of the exothermic reaction between MEA and $\mathrm{CO}_{2}$. A typical range for stripper column operation is 
approximately $90-120^{\circ} \mathrm{C}$, with the temperature increasing from top to bottom. The Aspen Plus library model generally underpredicts the data in the loading range below $0.6 \mathrm{~mol} \mathrm{CO} / \mathrm{MEA}$. This is due to the fact that this model's parameter regression considered a larger range of $\mathrm{CO}_{2}$ loading (approximately 0-1.4 mol $\mathrm{CO}_{2} / \mathrm{MEA}$ ), making the model accurate at higher loading at the cost of reducing accuracy in the loading range that is relevant for typical industrial applications. No data with loading greater than $0.6 \mathrm{~mol} \mathrm{CO}_{2} / \mathrm{MEA}$ are included in the regression for this work, ensuring increased accuracy over the relevant operating region of the postcombustion carbon capture process.

For the binary MEA- $\mathrm{H}_{2} \mathrm{O}$ system, phase envelope data corresponding to a water mole fraction greater than 0.6 are included in the regression, noting that the baseline $30 \mathrm{wt} \%$ MEA solution corresponds to a 0.8 mole fraction of water. These data are included in the regression in order to better capture the limiting behavior of the system as the $\mathrm{CO}_{2}$ concentration approaches zero. The comparison of the model to these data is shown in Figure 4.2.

A
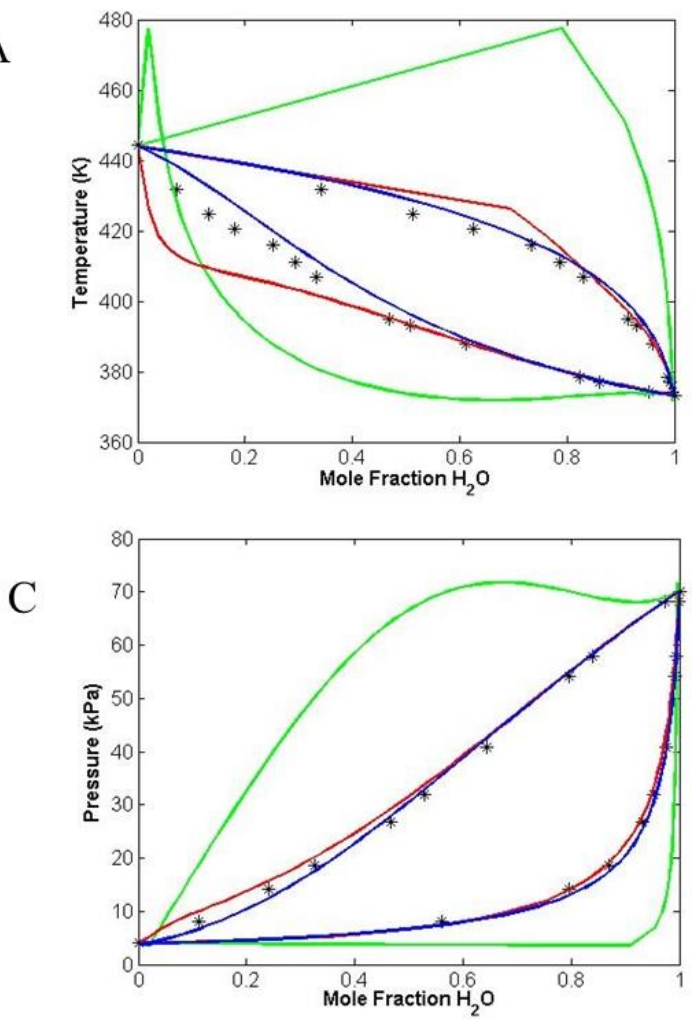

$\mathrm{B}$

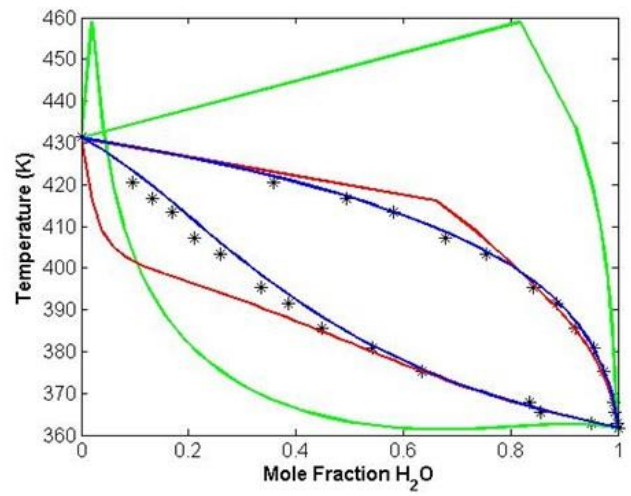

Figure 4-2. Comparison of phase envelope predictions to experimental data. T-xy data from Cai et al. (1996) are shown for fixed pressure of (A) $101.33 \mathrm{kPa}$ and (B) $66.66 \mathrm{kPa}$. Pxy data from Tochigi et al. (1999) are shown at fixed temperature of (C) $363.15 \mathrm{~K}$. 
Clearly, the Plaza (2012) model, which did not include these data in its parameter regression, gives an inadequate fit to the binary system data. By incorporating binary phase data in the new regression, the fit of the phase envelope data to the model improves markedly, particularly in the composition range of interest.

The fit of the heat of absorption data to the CCSI, Plaza (2012), and Aspen Plus library (Aspen Plus documentation, 2013a) models is shown in Figure 4.3. This includes differential heat of absorption data (Kim et al., 2014), which correspond to a solvent composition of $30 \mathrm{wt} \%$ MEA and temperature values of 40,80 , and $120^{\circ} \mathrm{C}$.

A

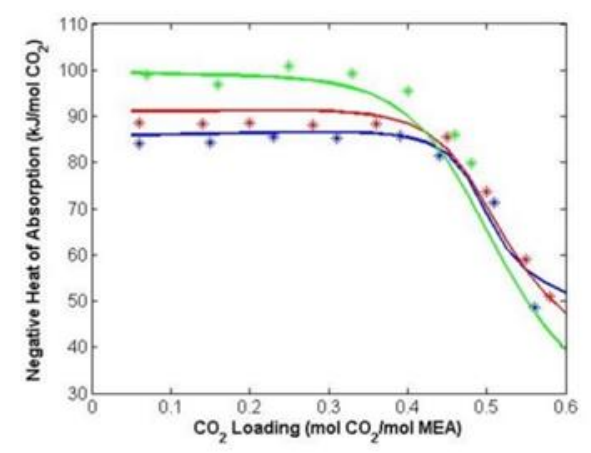

$\mathrm{C}$

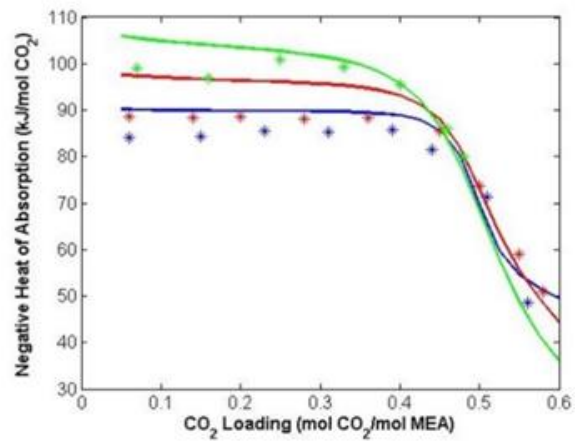

B

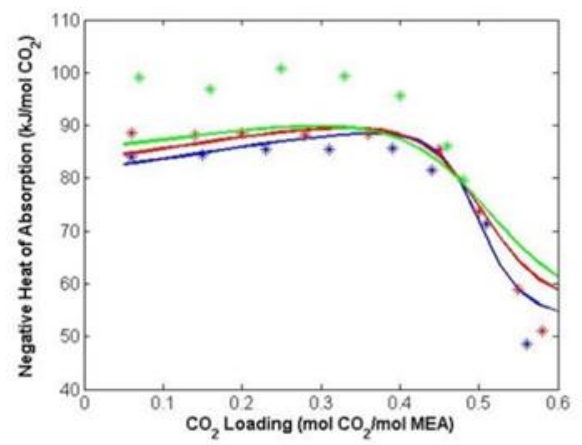

Figure 4-3. Heat of absorption data (Kim et al., 2014) for solutions of $30 \mathrm{wt} \% \mathrm{MEA}$ and temperatures of $40^{\circ} \mathrm{C}, 80^{\circ} \mathrm{C}$, and $120^{\circ} \mathrm{C}$ as a function of $\mathrm{CO}_{2}$ loading compared to model predictions for (A) CCSI, (B) Plaza, and (C) Aspen Plus library models.

It is shown that the temperature dependence of heat of absorption, as shown in the data, is not captured accurately in the Plaza model, which did not include these data in its regression. The fit of the data to the new model, however, clearly improves as a result of incorporating heat of absorption into the data regression through use of the FORTRAN user model. For the Aspen Plus library model, an earlier set of heat of absorption data (Kim and Svendsen, 2007), published by 
the same group as the data considered in this work, was included in the model regression. Although this model does capture the temperature dependence of the heat of absorption, it does not fit the new set of data as accurately as the CCSI model. The literature for the Aspen Plus library model (Aspen Plus documentation, 2013a) does not specify the details of its parameter regression, so it is unknown how the heat of absorption data were incorporated into the framework.

Finally, the heat capacity data included in the model regression (Weiland et al., 1997) correspond to a temperature of $25^{\circ} \mathrm{C}$ and solvent composition of 10-40 wt\% MEA. The comparison of the CCSI and Plaza models to the experimental data is presented in Figure 4.4, and the fit is shown to be adequate for the model developed in this work. The fit of data to the Aspen Plus library model is omitted from the figure for the purpose of clarity, but this model also fits the data accurately, and comparably to the CCSI model.

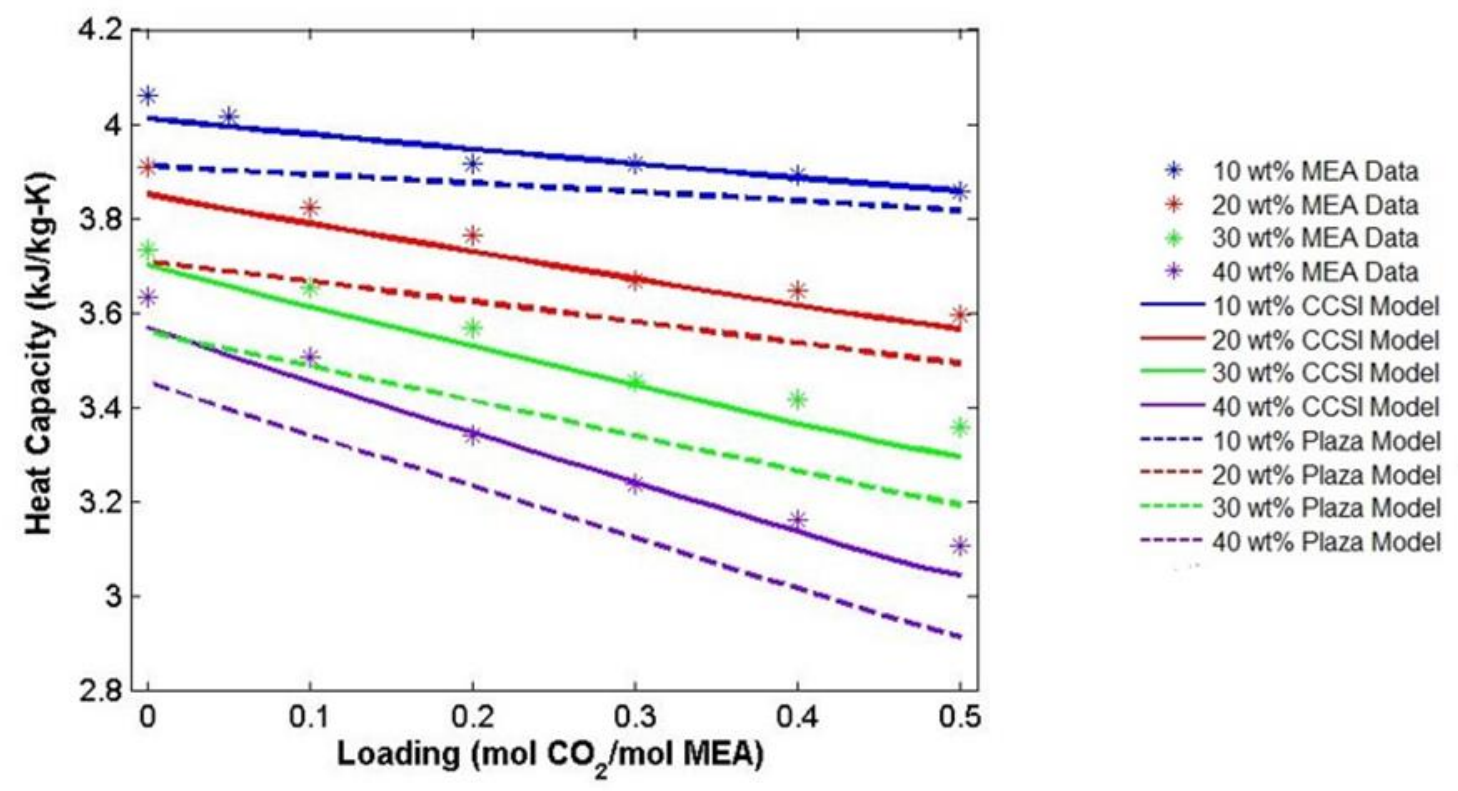

Figure 4-4. CCSI model and Plaza model predictions of heat capacity compared to data (Weiland et al., 1997) for solutions at $25^{\circ} \mathrm{C}$ for various values of MEA weight percent as a function of $\mathrm{CO}_{2}$ loading.

In summary, Tables 4.7-8 give comparison of the fit of the three models described in this work to the major sources of data included in the regression in this work. Table 4.7 gives the sum of square error (SSE) and Table 4.8 the values of the coefficient of determination $\left(R^{2}\right)$ of the model 
with respect to each type of data. These values are calculated using the full set of experimental data incorporated into the regression of each data type. For this analysis, the values of $\mathrm{CO}_{2}$ partial pressure are calculated as $\ln \left(\mathrm{P}_{\mathrm{CO}_{2}}\right)$.

Table 4-7. Values of SSE for models with respect to data included in the CCSI model regression

\begin{tabular}{cccc}
\hline Data Type & CCSI Model & $\begin{array}{c}\text { Aspen Plus }{ }^{\circledR} \text { Library } \\
\text { Model }\end{array}$ & Plaza Model \\
\hline $\mathrm{CO}_{2}$ Partial Pressure & 131 & 1080 & 276 \\
Heat Capacity & 0.037 & 0.191 & 0.448 \\
Heat of Absorption & 460 & 727 & 994 \\
\hline
\end{tabular}

Table 4-8. Values of $\mathbf{R}^{2}$ for models with respect to data included in the CCSI model regression

\begin{tabular}{cccc}
\hline Data Type & CCSI Model & $\begin{array}{c}\text { Aspen Plus }{ }^{\circledR} \text { Library } \\
\text { Model }\end{array}$ & Plaza Model \\
\hline $\mathrm{CO}_{2}$ Partial Pressure & 0.981 & 0.845 & 0.960 \\
Heat Capacity & 0.979 & 0.885 & 0.730 \\
Heat of Absorption & 0.925 & 0.882 & 0.838 \\
\hline
\end{tabular}

For each type of data, the overall SSE is lower for the new CCSI model in comparison to the existing models from Plaza (2012) and the Aspen Plus ${ }^{\circledR}$ library (Aspen Plus documentation, 2013a). Furthermore, all of the correlation coefficients between the model predictions and experimental data are highest for the CCSI model. Of all the models, only the CCSI model has been regressed with all of the data used to calculate the statistics in Tables 4.7-8.

Analysis of variance (ANOVA) is used to determine the statistical significance of the fit of the model to the experimental data. Since the overall model is used to represent three distinct dependent variables, the test is performed for each, with the results given in Tables 4.9-11. For each test, the null hypothesis states that the model regressed in this work is not more predictive of a dependent variable $(\varphi)$ than a generic model $\left(\varphi=\varphi_{0}\right)$, where $\varphi_{0}$ is a constant value. The null hypothesis is rejected at significance level $p$ under the condition that $F>F_{c}$, where $\mathrm{F}$ is the calculated value of the $\mathrm{F}$ statistic and $F_{c}$ is the critical value, for $\mathrm{p}$, of the F-distribution with degrees of freedom $k$ and $N-k-1$. Here, $k$ and $N$ refer to the number of parameters regressed and the number of data included of a given type, respectively. In Tables 4.9-11, DF, SS, and MS 
have their usual connotation. The values in Table 4.9 are calculated with respect to the transformed variable $\ln \left(P_{\mathrm{CO}_{2}}\right)$ to ensure that data observations with large values of $P_{\mathrm{CO}_{2}}$ do not dominate the calculations.

Table 4-9. ANOVA table for regression ( $\mathrm{CO}_{2}$ Partial Pressure)

\begin{tabular}{cccccc}
\hline & DF & SS & MS & F & $\mathrm{F}_{\mathrm{c}}(\mathrm{p}=0.05)$ \\
\hline Model & 10 & 6827 & 683 & 2550 & 1.85 \\
Error & 488 & 131 & 0.268 & & \\
Total & 498 & 6958 & & & \\
\hline
\end{tabular}

Table 4-10. ANOVA table for regression (Heat Capacity)

\begin{tabular}{cccccc}
\hline & DF & SS & MS & F & $\mathrm{F}_{\mathrm{c}}(\mathrm{p}=0.05)$ \\
\hline Model & 10 & 1.658 & 0.166 & 62.2 & 2.67 \\
Error & 13 & 0.0347 & 0.00267 & & \\
Total & 23 & & & & \\
\hline
\end{tabular}

Table 4-11. ANOVA table for regression (Heat of Absorption)

\begin{tabular}{cccccc}
\hline & DF & SS & MS & F & $\mathrm{F}_{\mathrm{c}}(\mathrm{p}=0.05)$ \\
\hline Model & 10 & 6155 & 615 & 21.4 & 2.49 \\
Error & 16 & 460 & 28.8 & & \\
Total & 26 & 6615 & & & \\
\hline
\end{tabular}

For all three tests, the null hypothesis is rejected at a significance level $p=0.05$ since all calculated values of the $F$ statistic exceed the respective critical values. Accordingly, the model has been shown to be predictive of all of the data types considered.

\subsection{Conclusions}

A deterministic model for the thermodynamic framework of the $\mathrm{MEA}-\mathrm{CO}_{2}-\mathrm{H}_{2} \mathrm{O}$ system has been developed, incorporating data for VLE, heat capacity, and heat of absorption that span the temperature and composition ranges of interest. This model has been developed using the eNRTL model in Aspen Plus ${ }^{\circledR}$ as a starting point, and the reaction kinetics are represented in a form that ensures consistency with the thermodynamic model. A parameter selection methodology that takes an information criterion (AIC) into account has been applied to 
determine an optimal set of parameters for representing the thermodynamic model. The model has been shown to give adequate fit to the various types of data over the ranges of interest for absorber and stripper columns in an MEA-based $\mathrm{CO}_{2}$ capture process. The uncertainty quantification of this model, along with the other property models considered in Chapter 3, is described in Chapter 5. 


\section{Chapter 5 : Uncertainty Quantification}

\subsection{Standalone Physical Property Models}

\subsubsection{Parameter Selection and Prior Selection}

The Bayesian inference approach may be computationally prohibitive, despite the use of surrogate response surface models, if the parameter space is too large. In addition, there may be parameters for which the uncertainty estimate is not improved with additional data, as their posterior distributions are approximately identical to the assumed prior distributions. The response surface corresponding to each parameter for each process variable can be visually examined to determine whether it can be excluded from UQ analysis. This approach can be very expensive for systems with a large number of parameters. For down-selecting the parameter space, we utilize a sensitivity matrix approach where the candidate parameters for UQ analysis can be automatically selected by constructing a matrix of partial derivatives of the property model with respect to the parameter value. The sensitivity matrix $S$ for a property $(\varphi)$ is of dimension $p \times q$ where a single element is given by:

$$
S_{i j}=\max _{x_{j} \in\left[x_{j}^{L}, x_{j}^{U}\right]}\left|\left(\frac{\partial \varphi}{\partial \widehat{\theta}_{i}}\right)_{x_{q \neq j}=x_{q}^{*}}\right|
$$

Here, $p$ and $q$ correspond to the number of parameters and variables, respectively, in the model and $x_{j}$ refers to a specific variable and $\hat{\theta}_{i}$ is a parameter deviation term defined by:

$$
\theta_{i}=\bar{\theta}_{i} \hat{\theta}_{i}
$$

where $\theta_{i}$ refers to a specific model parameter and $\bar{\theta}_{i}$ refers to the baseline value of that parameter, which is taken from the result of deterministic regression. The terms $x_{j}^{L}$ and $x_{j}^{U}$ represent the lower and upper limits of the variable value, determined by the ranges of the data used to develop the model, and $x_{q}^{*}$ represents the average of these two values for any given variable. The normalized version of this matrix $(N)$ is defined for a single element as:

$$
N_{i j}=\frac{S_{i j}}{\max _{i \in[1, p], j \in[1, q]} S_{i j}}
$$


For a given parameter $\theta_{k}$, if the condition $N_{k j} \ll 1 \forall j \in[1, q]$ is met, then the parameter is excluded from the UQ analysis as the prior belief of this parameter is unlikely to improve due to Bayesian inference. For this work, a parameter is excluded from UQ if its normalized sensitivity matrix values are less than 0.1 for all variables. This methodology is performed for the viscosity, molar volume, and surface tension models in the subsequent sections of this work. The procedure is validated qualitatively by comparing the calculated derivatives with response surfaces generated using MARS.

For the standalone physical property models, the prior distributions of the model parameters used for Bayesian inference are determined by estimating confidence intervals for the parameters taken from the results of the deterministic regression. The prior distributions of the parameters are determined by estimating their probability density functions (pdf) from a series of confidence intervals. A confidence interval of significance level $\gamma$ for the parameter $\theta$ may be expressed as:

$$
P\left(\theta_{\gamma}^{L} \leq \theta \leq \theta_{\gamma}^{U}\right)=1-\gamma
$$

where $\theta_{\gamma}^{L}$ and $\theta_{\gamma}^{U}$ are the lower and upper limits of the parameter for a confidence interval of $100(1-\gamma) \%$. Parameters for which the value 0 is contained in the $95 \%$ confidence interval are not considered for UQ, along with those eliminated in the sensitivity matrix methodology. From the confidence interval, the cumulative distribution function (CDF) of the parameter may be determined by:

$$
\begin{gathered}
F_{\theta}\left(\theta_{\gamma}^{L}\right)=\frac{\gamma}{2} \\
F_{\theta}\left(\theta_{\gamma}^{U}\right)=1-\frac{\gamma}{2}
\end{gathered}
$$

An estimate of the entire CDF of each parameter is obtained by calculating all confidence intervals for levels $\gamma=0.01-0.99$ at increments of 0.01 . The PDF of each parameter is determined from a numerical approximation of the derivative:

$$
f_{\theta}(\theta)=\frac{d F_{\theta}(\theta)}{d \theta}
$$

For the overall joint prior parameter distribution for each of the models, independence of the parameters is assumed, although this condition does not necessarily hold for the posterior 
distribution. For each physical property, the resulting posterior distribution is given in the form of one joint distribution.

\subsubsection{Viscosity Model}

For the viscosity model, the sensitivity matrix is determined by considering the predictor variable ranges of:

$$
\begin{gathered}
20 \leq W_{M E A}^{*} \leq 40 \\
298.15 \leq T(K) \leq 353.15 \\
0 \leq \alpha \leq 0.5
\end{gathered}
$$

These ranges are determined based on the ranges of the data used to regress the model parameters. The normalized sensitivity matrix is given as:

$$
N=\max \left[\begin{array}{c|c|c}
\mid\left(\frac{\partial \mu}{\partial \hat{a}}\right)_{W_{M E A}^{*}, \alpha} & \left|\left(\frac{\partial \mu}{\partial \hat{a}}\right)_{T, \alpha}\right| & \mid\left(\frac{\partial \mu}{\partial \hat{a}}\right)_{T, W_{M E A}^{*}} \\
----- & --- & --- \\
---- & --
\end{array} \mid=\left[\begin{array}{ccc}
0.3383 & 0.4601 & 0.2667 \\
0.3877 & 0.3955 & 0.3057 \\
0.4556 & 0.5673 & 0.3289 \\
0.8200 & 0.7658 & 0.5919 \\
0.0682 & 0.0811 & 0.0757 \\
0.8244 & 0.8023 & 1.0000 \\
0.6413 & 0.5714 & 0.7122
\end{array}\right]\right.
$$

Eq. 5.7 shows the normalized sensitivity of the viscosity model to its parameters, evaluated over the range of interest for each variable. The model is clearly most sensitive to parameter $f$, since the corresponding row of the matrix has the highest value for all three columns, and least sensitive to parameter $e$. To confirm the validity of these results, a response surface is generated using MARS with a sample size of 4000 with variable and parameter inputs sampled from a Monte Carlo simulation with uniform distributions (ranges from $\pm 10 \%$ of the baseline values for parameters and variable ranges given in Eq. 5.7) and viscosity output calculated from Eq. 3.1. Figure 5.1 shows three-dimensional projections from this response surface representing viscosity as a function of $\mathrm{CO}_{2}$ loading and one of the model parameters. All plots are generated at $T=325.65 K$ and $W_{M E A}^{*}=30$ (the average values over the variable ranges of interest) and at baseline parameter values given in Table 3.1, except for the varied parameter of interest for a given plot. 

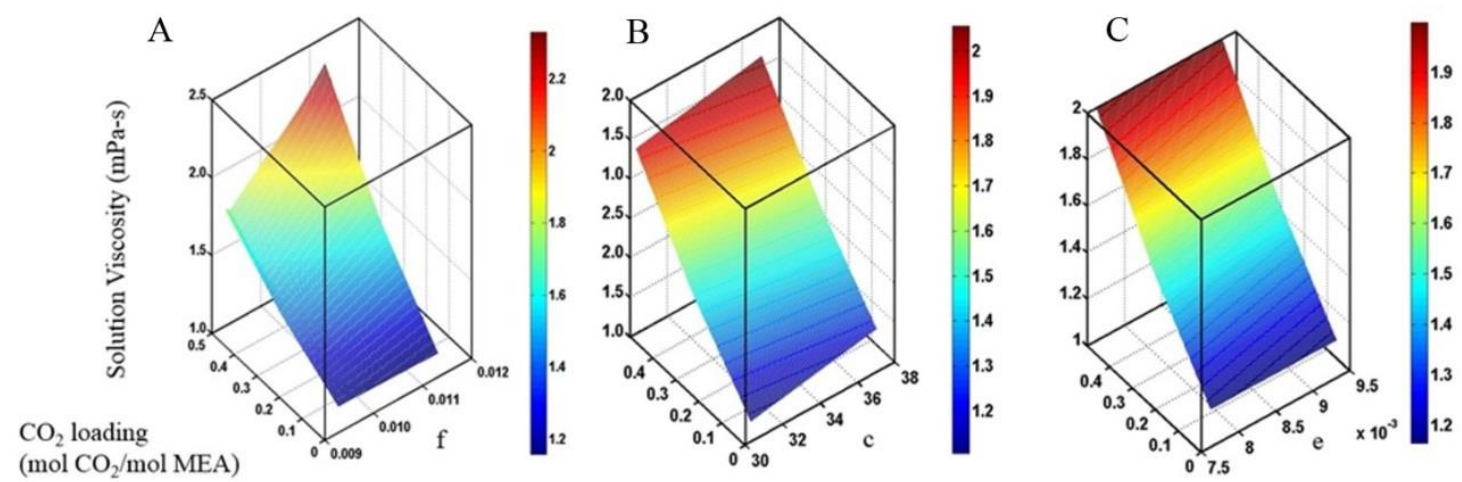

Figure 5-1. Response surfaces for viscosity model representing: A) $\mu_{\operatorname{sln}}=\mu_{\operatorname{sln}}(f, \alpha)$;

B) $\mu_{s \ln }=\mu_{s \ln }(c, \alpha)$; C) $\mu_{s \ln }=\mu_{\operatorname{sln}}(e, \alpha)$

The relatively high sensitivity of the viscosity model with respect to parameter $f$ is confirmed by the shape of the curve in Figure 5.1A, which depicts large deviation in the viscosity prediction, particularly at high loading values, as a result of a small perturbation in the value of the parameter. There is clearly little sensitivity of the viscosity model to parameter $e$ (Figure 5.1C), as the cure representing viscosity as a function of loading changes negligibly with the parameter value. It can be noted that $N_{e j} \ll 1 \forall j \in[1,3]$. The parameter $c$ is considered as an intermediate case (noting $N_{e \alpha}<N_{c \alpha}<N_{f \alpha}$ as given by Eq. 5.8), and the reponse surface of viscosity to this parameter (Figure 5.2B) is shown to be more subtly peaked than that of parameter $f$. This study shows that the sensitivity matrix approach can be effectively used to avoid unnecessary UQ analysis for parameters without examining the response surfaces visually. Based on the sensitivity matrix calculation, the parameter $e$ is not considered further for uncertainty quantification.

For the remaining parameters, the calculated $95 \%$ confidence intervals are given in Table 5.1. 
Table 5-1. Calculated 95\% confidence intervals for parameters in viscosity model

\begin{tabular}{ccc}
\hline Parameter & Baseline Value & Confidence Interval \\
\hline a & -0.0838 & {$[-0.2341,0.0666]$} \\
b & 2.8817 & {$[-3.1179,8.8814]$} \\
c & 33.651 & {$[-13.6178,80.9212]$} \\
d & 1817 & {$[-66.8772,3701.13]$} \\
f & 0.0103 & {$[0.0071,0.0135]$} \\
g & -2.3890 & {$[-3.4216,-1.3565]$} \\
\hline
\end{tabular}

For the viscosity model, the only parameters considered for UQ are $f$ and $g$, as these are the parameters for which the value 0 is not contained in the $95 \%$ confidence interval. The prior distributions are calculated from the estimation of a series of confidence intervals, as described in Section 5.1.1. The CDFs and PDFs of the two parameters are given in Figure 5.2.
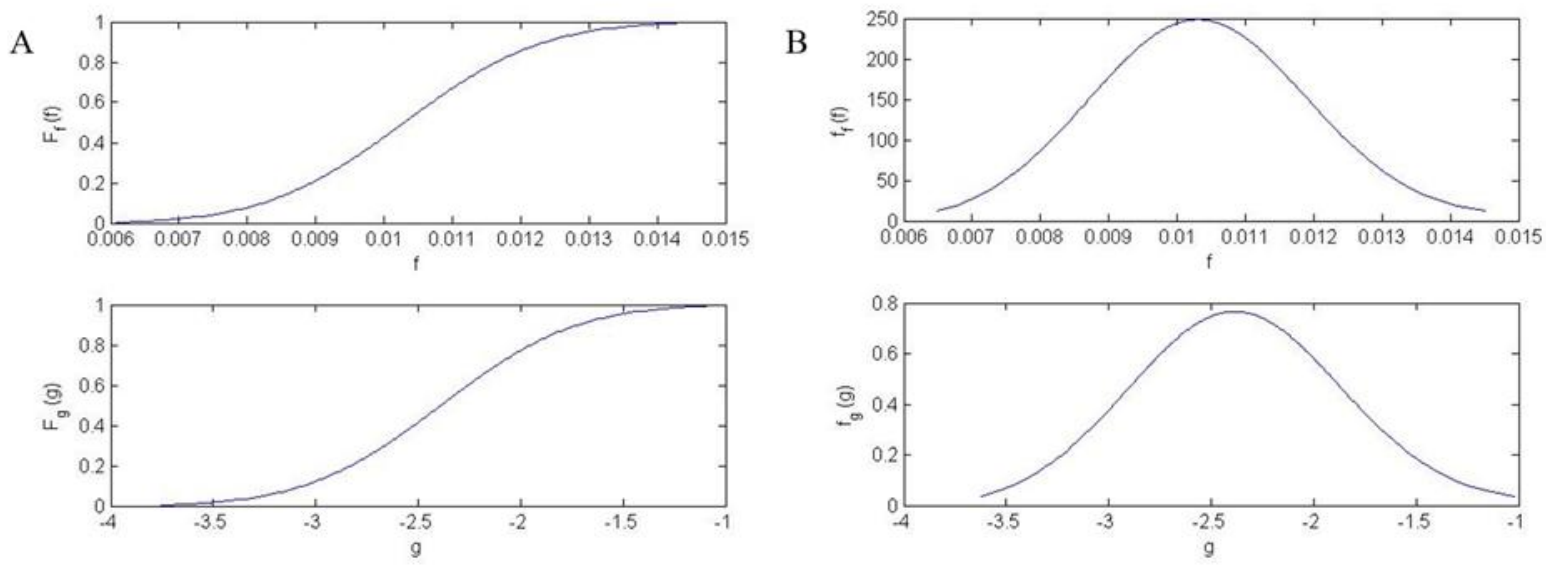

Figure 5-2. Approximation of (A) cumulative distribution functions and (B) probability distribution functions for viscosity models parameters determined from confidence intervals in the deterministic regression results

The marginal prior distributions of the model parameters are taken as the PDFs given in Figure 6.2 , and the joint prior distribution is determined by assuming independence of the parameters. For UQ of the viscosity model, a sample of 100 is taken from the distribution of each of the parameters. These parameter sets are concatenated and combined with 90 sets of independent variables (combinations of $\mathrm{T}(\mathrm{K}), W_{M E A}^{*}$, and $\alpha$ ) which correspond to the conditions at which the 
experimental data are available. Therefore, a total of 9000 model predictions is used to develop the response surface used for Bayesian inference. This response surface is validated by a 10-fold cross validation procedure, which results in a coefficient of determination of $R^{2}=0.9403$. The posterior distribution is obtained from Bayesian inference as a set of sample points. Figure 5.3 shows the posterior distributions in terms of marginal PDFs in histogram form and a contour plot that represents the joint probability distribution of the two parameters.
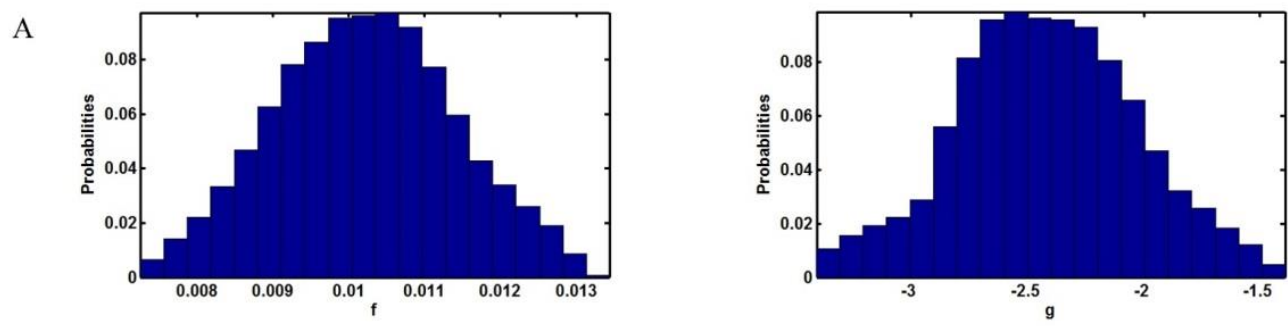

$\mathrm{B}$

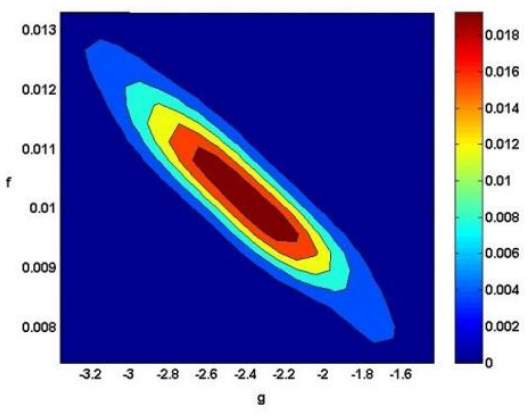

Figure 5-3. Posterior distributions for viscosity model parameters obtained from Bayesian inference. (A) Histograms representing marginal distributions of the parameters; (B) contour plot representing joint distribution.

By comparing the marginal posterior distributions in Figure 5.3A with the marginal prior distributions in Figure 5.2A, the reduction in parameter space resulting from Bayesian inference is not apparent. The contour plot in Figure 5.3B representing joint distribution $f_{f, g}(f, g)$, however, does clearly demonstrate that the prior distribution assumption that the parameters are independent random variables does not hold for the posterior distribution. Accordingly, the feasible parameter space in the joint distribution gets reduced while performing Bayesian inference.

Figure 5.4 shows the stochastic model predictions for $30 \mathrm{wt} \%$ MEA solution as a function of $\mathrm{CO}_{2}$ loading and temperature with the parameter distribution represented by both the prior and the posterior. All comparisons are made by drawing a sample of size 50 from the parameter 
distributions of interest. All parameters not included in UQ are treated as the constant values given in Table 3.1.
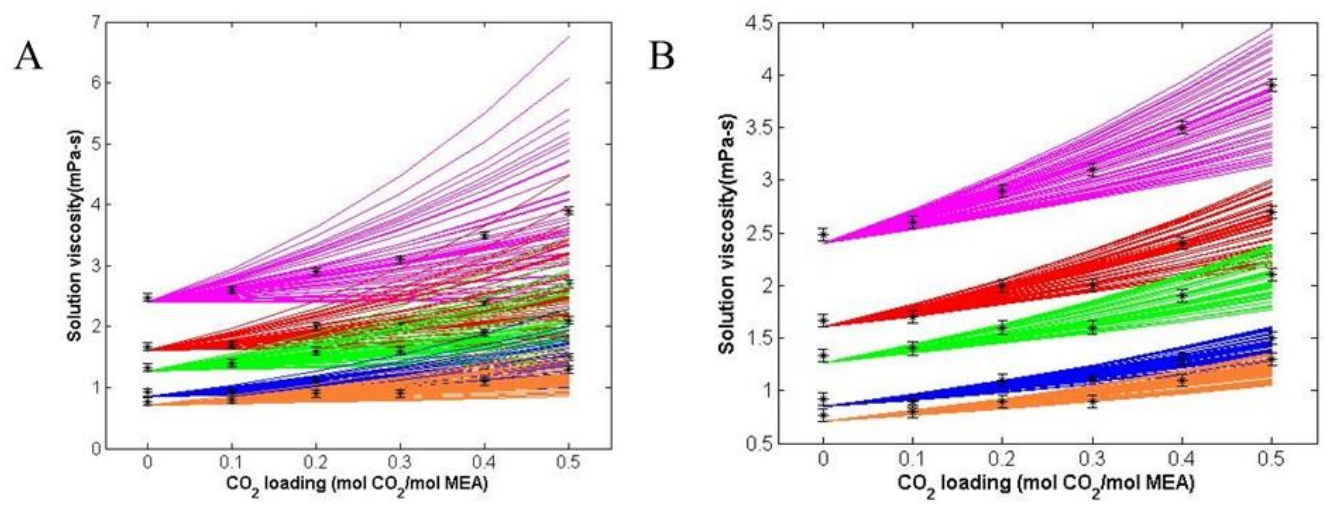

= $298.15 \mathrm{~K}$

- $313.15 \mathrm{~K}$

$=323.15 \mathrm{~K}$

- $343.15 \mathrm{~K}$

$=353.15 \mathrm{~K}$

Figure 5-4. Stochastic viscosity model for $\mathrm{W}_{\mathrm{MEA}}=30 \%$ considering $(\mathrm{A})$ prior parameter distribution and (B) posterior parameter distribution. Experimental data represented by (*) and taken from Amundsen et al. (2009)

In Figure 5.4, it is shown that the viscosity ranges predicted from the stochastic model with the prior distribution are excessively large, and the ranges are reduced considerably as a result of Bayesian inference. Furthermore, it is clear that the two parameter stochastic model is adequate for characterizing the uncertainty of the model.

\subsubsection{Molar Volume Model}

The sensitivity matrix is calculated for the molar volume model by the same procedure used for the viscosity model. The parametric sensitivity is calculated with respect to the molar volume (Eq. 3.13) rather than the density because derivatives of molar volume can be calculated analytically. Since the density is dependent on the solution molecular weight, which is calculated based on the electrolyte speciation of the system, its derivatives cannot be obtained analytically. The molecular weight calculation is independent of the model parameters, so the relative importance of these parameters can be determined by examining the sensitivity of either density or molar volume to them. The calculated sensitivity matrix is given as: 


$$
N=\max \left[\begin{array}{c|c|c}
\mid\left(\frac{\partial V}{\partial \hat{a}}\right)_{w_{M E A}^{*}, \alpha} & \left|\left(\frac{\partial V}{\partial \hat{a}}\right)_{T, \alpha}\right| & \mid\left(\frac{\partial V}{\partial \hat{a}}\right)_{T, w_{M E A}^{*}} \\
---- & --- \\
--- & --- & --- \\
\mid\left(\frac{\partial V}{\partial \hat{e}}\right)_{w_{M E A}^{*}, \alpha} & \left|\left(\frac{\partial V}{\partial \hat{e}}\right)_{T, \alpha}\right| & \left|\left(\frac{\partial V}{\partial \hat{e}}\right)_{T, w_{M E A}^{*}}\right|
\end{array} \mid=\left[\begin{array}{ccc}
0.2174 & 0.3139 & 0.4220 \\
0.1482 & 0.1987 & 0.1577 \\
0.0214 & 0.0415 & 0.0235 \\
0.4797 & 1.0000 & 0.9035 \\
0.142 & 0.4276 & 0.2596
\end{array}\right]\right.
$$

As for the viscosity model, these derivatives are evaluated over the variable ranges used to develop the deterministic models. Based on the relative values of the matrix elements, the parameter to which the model is most sensitive is $d$. Parameter $c$ is excluded from Bayesian inference because all elements in the respective row are sufficiently close to zero.

Further parameter selection is considered by estimating the $95 \%$ confidence intervals of the parameters, and the values are shown in Table 5.2.

Table 5-2. Calculated $95 \%$ confidence intervals for parameters in molar volume model

\begin{tabular}{ccc}
\hline Parameter & Baseline Value & Confidence Interval \\
\hline a & 10.2074 & {$[4.6508,15.764]$} \\
b & -2.2642 & {$[-3.0579,-1.4704]$} \\
d & 207 & {$[115.1532,298.8448]$} \\
e & -563.3701 & {$[-941.8307,-184.9095]$} \\
\hline
\end{tabular}

Since none of the four parameters contain 0 in their confidence intervals, all are considered in the stochastic model. The marginal prior distributions are determined using the previously discussed methodology, and the CDFs and PDFs are given in Figure 5.5. 
A
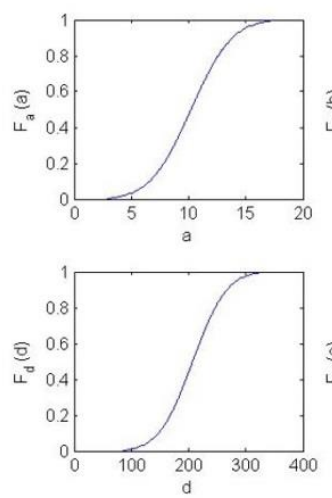
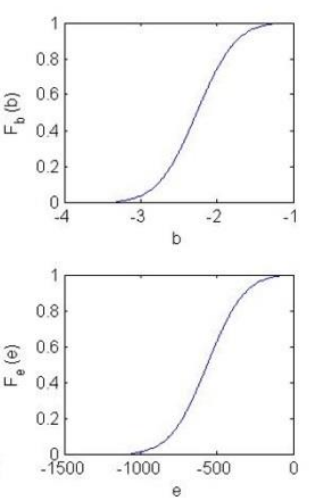

B
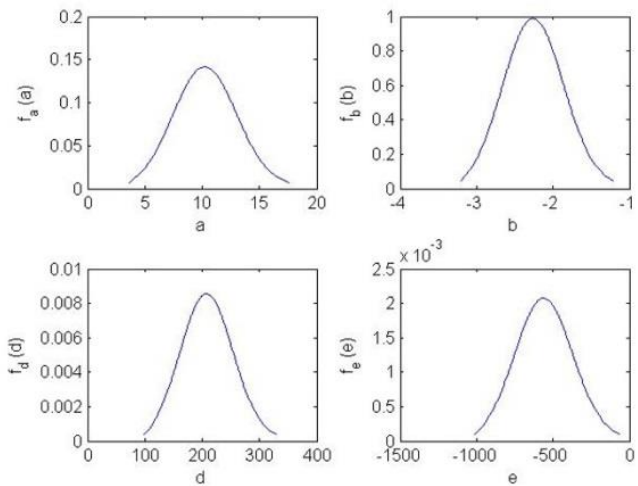

Figure 5-5. Approximations of (A) cumulative distribution functions and (B) probability distribution functions for density model parameters determined from the confidence intervals in the deterministic regression results.

The marginal prior distributions of the model parameters are taken as the PDFs given in Figure 5.5, and the joint prior distribution is determined by assuming independence of the parameters. A sample of 100 parameter sets is obtained from a numerical approximation of the PDFs. These sets are combined with 209 sets of independent variables which correspond to the conditions at which experimental data are available. Therefore, 20900 model predictions are used to develop the response surface model, which is validated by a 10-fold cross validation procedure that yields a value of $R^{2}=0.9999$. Figure 5.6 shows a representation of the posterior distribution in terms of histograms for all single parameter marginal distributions and contour plots for selected two parameter marginal distributions. 
A
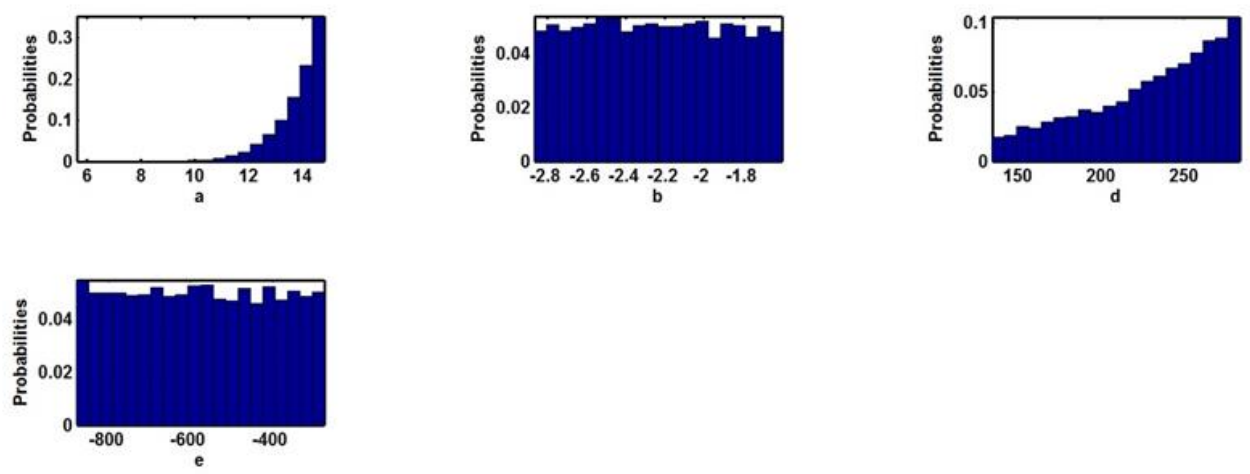

B
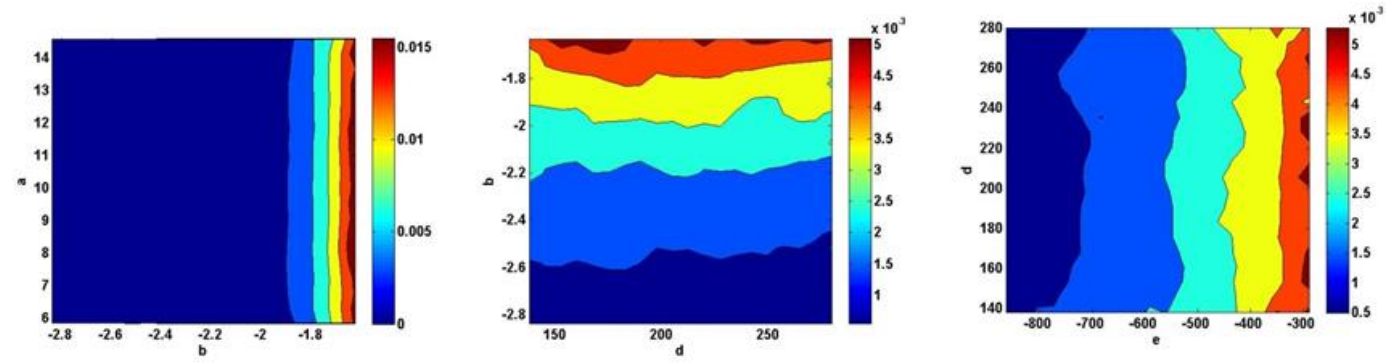

Figure 5-6. Posterior distributions for density model parameters obtained from Bayesian inference. (A) Histograms representing single parameter marginal distributions of the parameters; (B) Contour plots representing two parameter marginal distributions.

As the parameter space of the density model has four dimensions, it cannot be visualized directly as with the viscosity model. The histograms in Figure 5.6A represent the marginal distribution of single parameters, and it should be noted that the major change that occurs as a result of Bayesian inference is that the probability mass density of parameters $a$ and $d$ is shifted toward the upper boundary of the $95 \%$ confidence interval.

\subsubsection{Surface Tension Model}

The sensitivity matrix for the surface tension model is evaluated using the same criteria used for the viscosity and molar volume models. It is calculated as: 


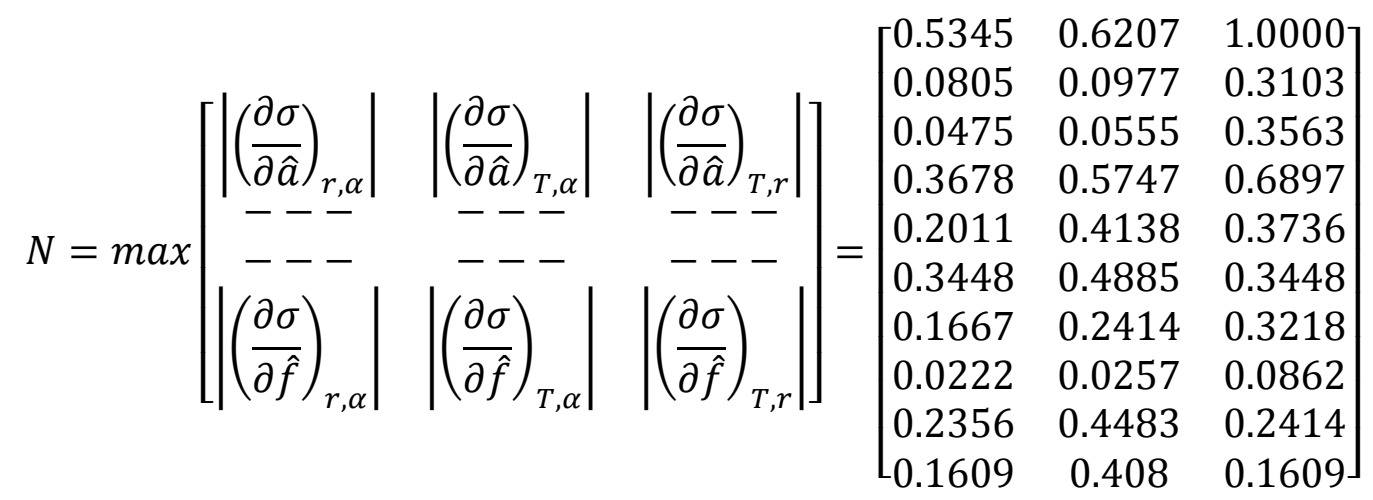

Given the results of the sensitivity matrix, the only parameter that can obviously be eliminated before Bayesian inference is $h$ because all elements in the corresponding row are sufficiently close to zero (less than 0.01).

The estimated $95 \%$ confidence intervals for the surface tension model, excluding parameter $h$, are given in Table 5.3.

Table 5-3. Calculated 95\% confidence intervals for parameters in surface tension model

\begin{tabular}{ccc}
\hline Parameter & Baseline Value & Confidence Interval \\
\hline $\mathrm{a}$ & 2.4558 & {$[1.5100,3.4015]$} \\
$\mathrm{b}$ & -1.5311 & {$[-3.4461,0.3840]$} \\
$\mathrm{c}$ & 3.4994 & {$[1.9749,5.0239]$} \\
$\mathrm{d}$ & -5.6398 & {$[-9.7085,-1.5711]$} \\
$\mathrm{e}$ & 10.2109 & {$[3.5877,16.8341]$} \\
$\mathrm{f}$ & 2.3122 & {$[1.2025,3.4219]$} \\
$\mathrm{g}$ & 4.5608 & {$[0.7945,8.3271]$} \\
$\mathrm{i}$ & 5.3324 & {$[-1.9564,12.6211]$} \\
$\mathrm{j}$ & -12.0494 & {$[-23.5634,-0.5355]$} \\
\hline
\end{tabular}

Parameters $b$ and $i$ are omitted from the stochastic model as the value 0 is included in their $95 \%$ confidence intervals. The prior distributions of the remaining seven parameters are estimated using the aforementioned methodology, and the marginal PDFs and CDFs are given in Figure 5.7 . 
A
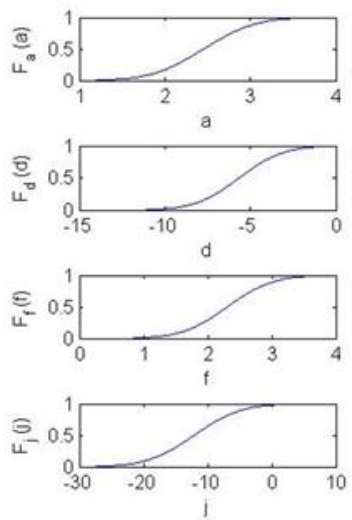
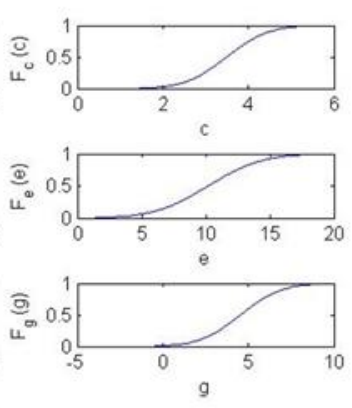

$\mathrm{B}$
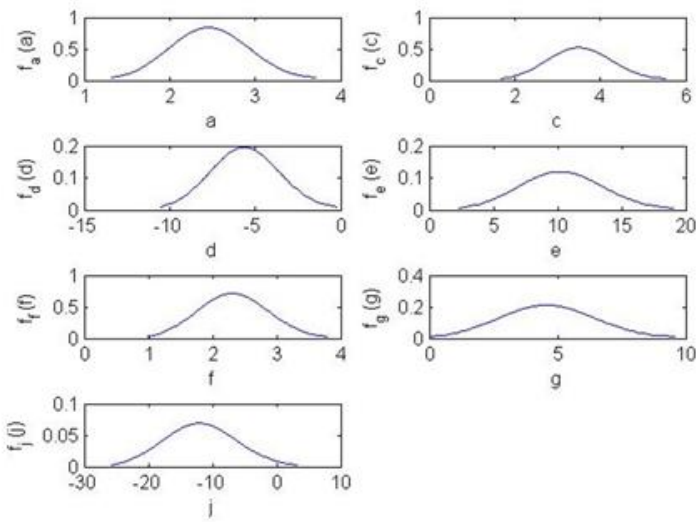

Figure 5-7. Approximations of (A) cumulative distribution functions and (B) probability distribution functions for surface tension model parameters determined from the confidence intervals in the deterministic regression results.

The marginal prior distributions are taken as the PDFs given in Figure 5.7, and the joint prior distribution is determined by assuming independence of the parameters. For UQ of the surface tension model, a sample of 100 parameter sets is obtained from a numerical approximation of the PDFs. This sample is combined with 72 sets of independent variables based upon those for which experimental data are included in the parameter regression, so that 7200 model observations are available for generating the response surface model, which is validated by a 10fold cross validation procedure with $R^{2}=0.971$. Figure 5.8 shows a representation of the posterior distribution in terms of histograms for all single parameter marginal distributions and contour plots for selected two parameter marginal distributions. 
A
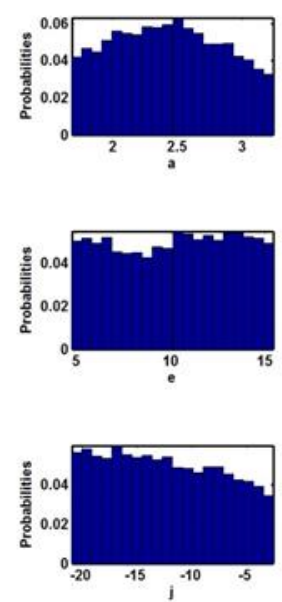

B

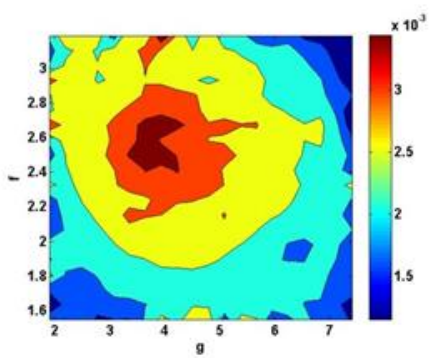

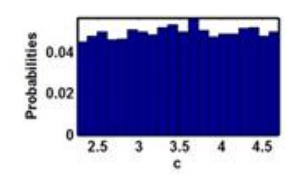
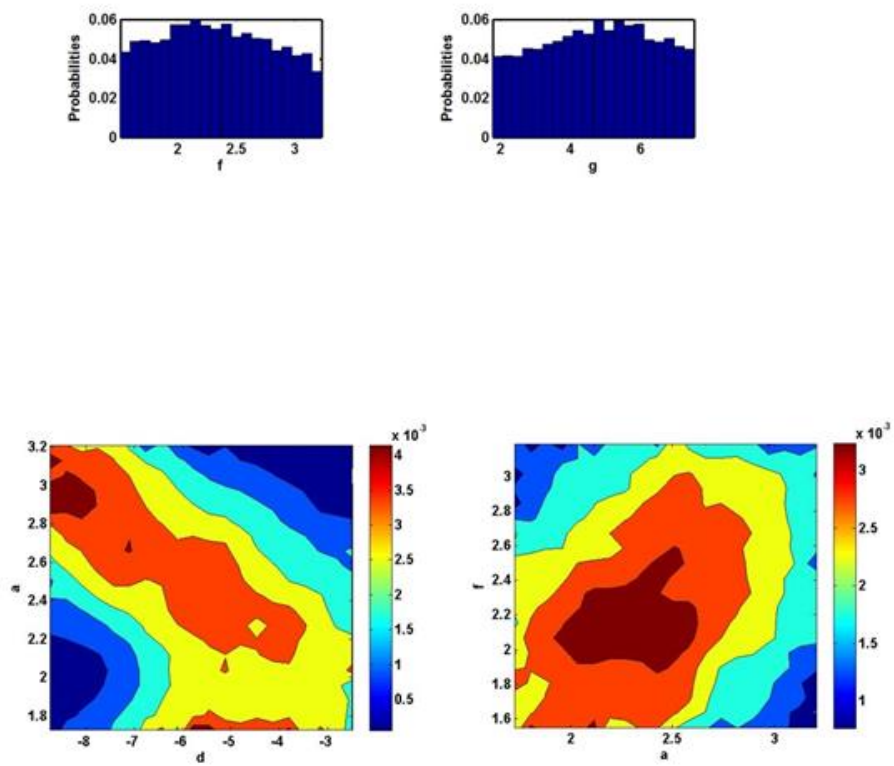

Figure 5-8. Posterior distributions for surface tension model parameters obtained from Bayesian inference. (a) Histograms representing single parameter marginal distributions of the parameters; (B) Contour plots representing two parameter marginal distributions.

As with the molar volume model, the entire joint posterior distribution cannot be visualized directly.

\subsection{Thermodynamic Framework}

A multivariate normal distribution is used as the prior distribution for the thermodynamic model, so that the joint pdf is given by (Hogg and Craig, 1978):

$$
f(\tilde{\theta})=\frac{1}{(2 \pi)^{\frac{n}{2}}|\Sigma|^{\frac{1}{2}}} \exp \left\{-\frac{1}{2}(\tilde{x}-\tilde{\mu})^{T} \Sigma^{-1}(\tilde{x}-\tilde{\mu})\right\}
$$

where $\tilde{x}$ is a random vector of dimension $n$ with mean values $\tilde{\mu}$ and covariance matrix $\Sigma$. The regression output of the final deterministic model, as given in Tables 4.4-5, is used to determine the hyperparameters (parameters of the prior distribution) for the UQ analysis. The means are taken directly and covariance matrix is calculate from the matrix of correlation coefficients and the parameter standard deviations. A total of nine parameters is included in this distribution; the 
parameter denoted as No. 9 in Table 4.4 is eliminated from UQ analysis and fixed at its constant deterministic value due to the fact that its standard deviation is larger than the absolute value of its mean. This criterion is comparable to the omission of parameters for which the value zero is included in the 95\% confidence intervals for the models described in Chapter 5.1. Moreover, it has been determined that inclusion of this parameter while sampling from this distribution results in infeasible model output for many sample points.

For developing the stochastic model, a subset of the experimental data is chosen for inclusion in the Bayesian inference methodology. A representative subset of the data, rather than the full set of data, is used to reduce the computational expense of Bayesian inference. The $\mathrm{CO}_{2}$ partial pressure for $30 \mathrm{wt} \%$ MEA solutions at temperatures of 40,80 , and $120^{\circ} \mathrm{C}$ and the full loading range (0.0001-0.6 mol CO $2 / \mathrm{MEA})$ are chosen because a majority of the data are given at these conditions, and they are representative of the standard process conditions for $\mathrm{CO}_{2}$ capture applications. A sample of size 5000 is taken from the joint prior distribution and combined with a sample of the same size of $\mathrm{CO}_{2}$ loading values taken from a uniform distribution $(U(0.0001,0.6))$ with upper and lower value parameters corresponding to the range of interest. For each of the three temperature values of interest, this sample is propagated through the process simulation to calculate $\mathrm{CO}_{2}$ partial pressure. Some minor modifications to the prior distribution was carried out to ensure that the predicted ranges of the dependent variable cover the experimental data over the ranges of all independent variables of interest. The evaluation of $\mathrm{CO}_{2}$ partial pressure as a function of temperature and loading for the stochastic model is shown in Figure 5.9. 
A
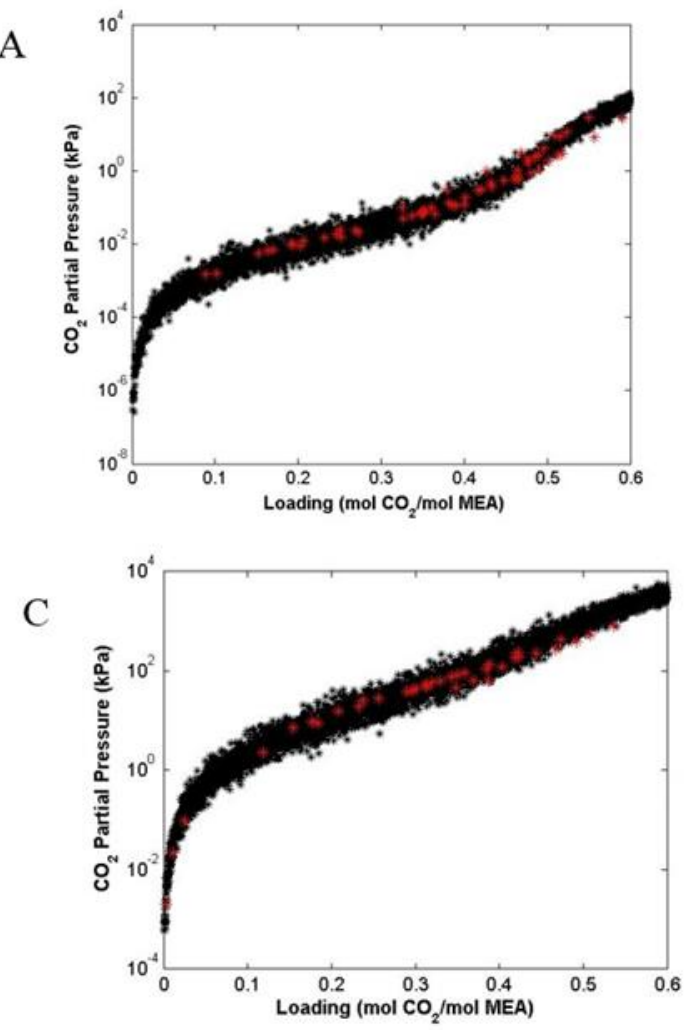

B

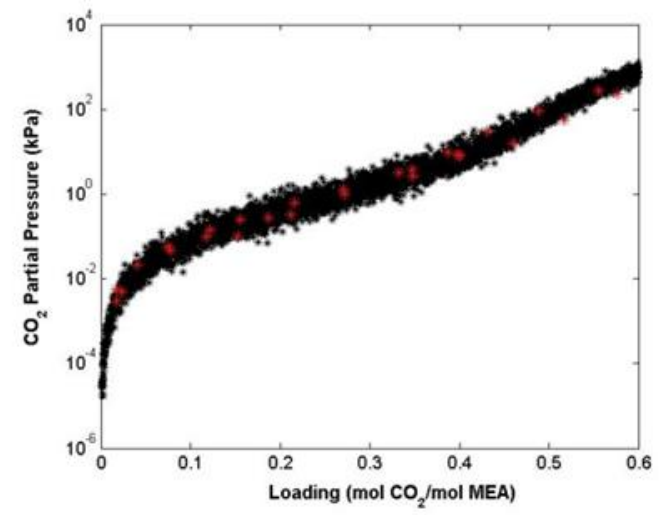

* Model

* Data

Figure 5-9. $\mathrm{CO}_{2}$ partial pressure prediction for stochastic model with a modified prior parameter distribution with experimental data for $30 \mathrm{wt} \%$ MEA at temperatures of (a) $40^{\circ} \mathrm{C}$, (b) $80^{\circ} \mathrm{C}$, and (c) $120^{\circ} \mathrm{C}$.

The Bayesian inference is performed by first developing the response surface model and then using it with the experimental data with their estimated standard deviation. For this procedure, the values of $P_{\mathrm{CO}_{2}}$ are transformed to $\ln \left(\mathrm{P}_{\mathrm{CO}_{2}}\right)$ to improve the quality of the response surface model, particularly with respect to avoidance of negative partial pressure predictions. The accuracy of the reponse surface model is tested by a cross validation procedure, given that accurate estimation of the posterior distribution is contingent upon the use of a reponse surface that adequately emulates the original model. The results of this procedure are shown in Figure 5.10 . 

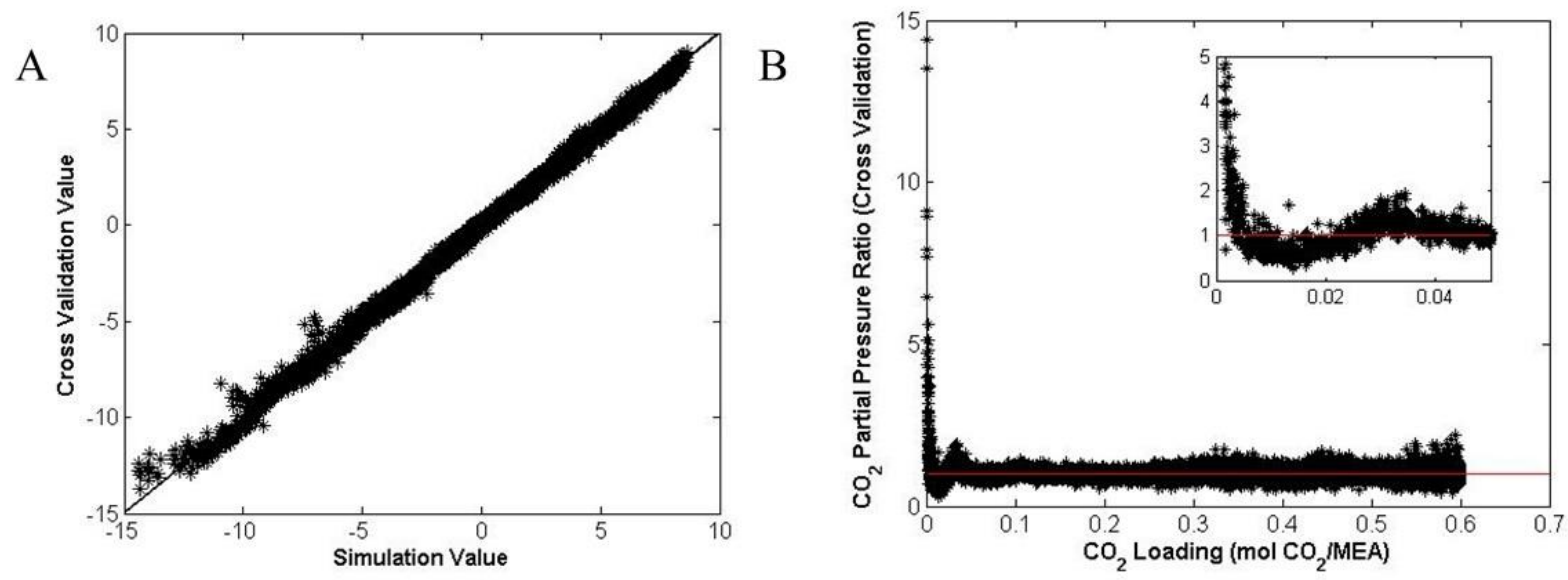

Figure 5-10. Quality of response surface model for $\mathrm{CO}_{2}$ partial pressure. (A) Parity plot for cross validation (10-fold) of response surface model in comparison to simulation values. Values shown correspond to $\ln \left(\mathrm{P}_{\mathrm{CO}_{2}}[\mathrm{kPa}]\right)$. (B) Ratio of response surface predictions to simulation values for $\mathrm{CO}_{2}$ partial pressure (without logarithmic transformation) as a function of $\mathrm{CO}_{2}$ loading.

As shown in Figure 5.10, the reponse surface model developed with MARS serves as an effective surrogate for the actual model, and the actual simulation values and response surface values are correlated with a value of $R^{2}=0.9983$. From Figure 5.10B, it is clear that the response surface model loses accuracy only for very low $(<0.01)$ values of lean loading. Accordingly, the validity of the stochastic model, which is represented by the resulting posterior parameter distribution, must be considered questionable in this regime. However, process operation at such a low value of lean loading is generally impractical due to the large cost associated with the energy input into the stripper reboiler, and operation below 0.1 mol $\mathrm{CO}_{2}$ /MEA is generally not recommended (Kohl and Nielsen, 1997). In replacing the full thermodynamic model with the response surface model, the computational cost associated with running thousands of Aspen Plus® simulations to evaluate $\mathrm{CO}_{2}$ partial pressure as a function of the model parameters and predictor variables is greatly reduced.

The prior and posterior distributions are fully represented by a 9 parameter joint distribution that cannot be visualized readily, although some representation of the change in the parameter distribution resulting from UQ is shown in Figures 5.11 and 5.12. Single parameter marginal distributions for all parameters are shown in Figure 5.11 and selected examples of two parameter marginal distributions are shown in Figure 5.12. For the purpose of clarity, the distributions 
shown in Figures 5.11-12 are for normalized parameter values; the value of each parameter $\theta_{i}$ is replaced by a normalized value:

$$
\theta_{i}^{*}=\frac{\theta_{i}}{\hat{\theta}_{i}}
$$

where $\hat{\theta}_{i}$ is the deterministic model value for the given parameter, as given in Table 5.4.
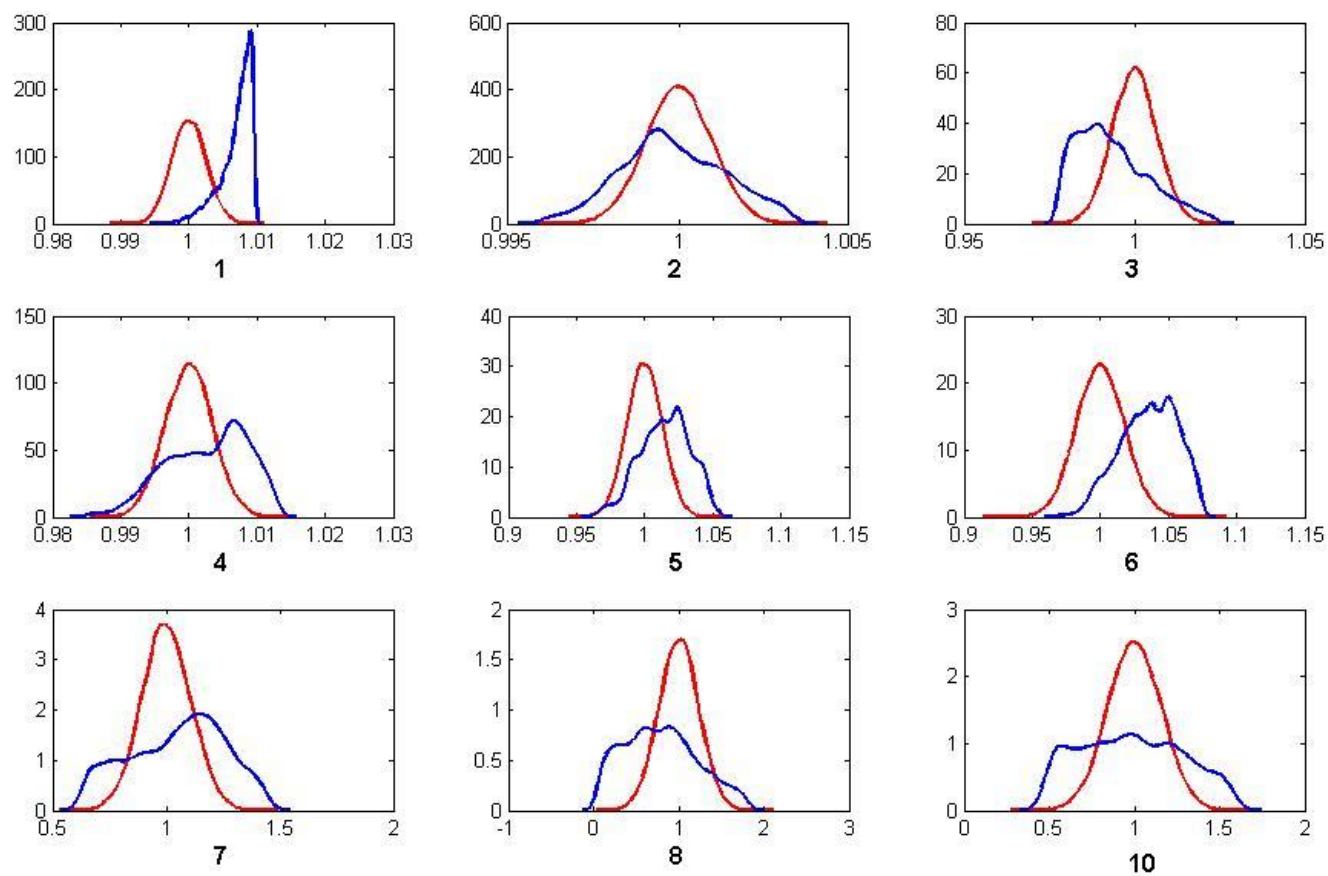

Figure 5-11. Single parameter marginal probability density functions for prior and posterior distributions. Red and blue lines represent prior and posterior distributions, respectively, and parameter numbers match those given in Table 4.4. Parameter values are normalized by dividing by the deterministic value of the respective parameter. 

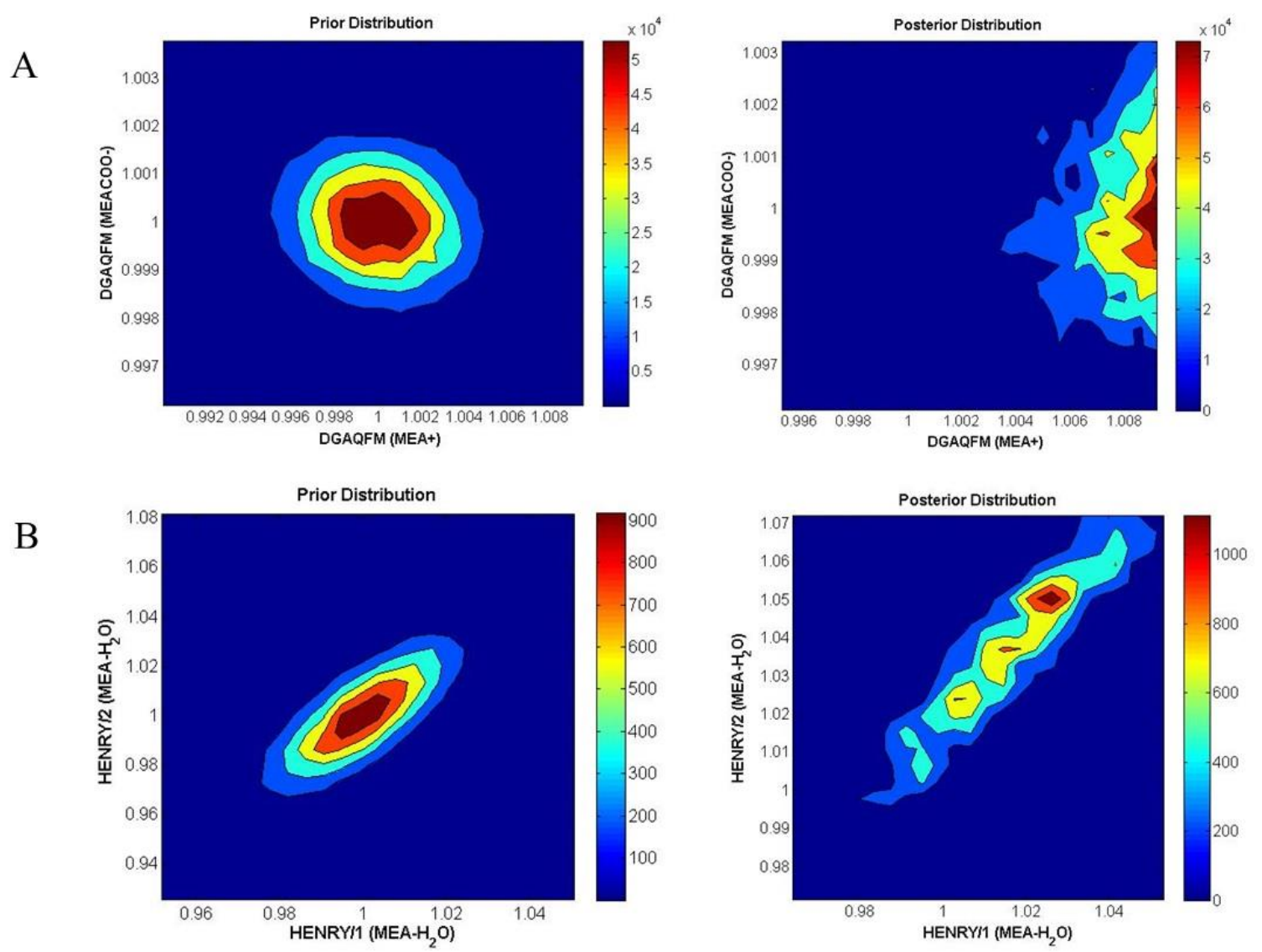

Figure 5-12. Normalized prior and posterior two-parameter marginal distributions for (A) parameters 1 and 2 and (B) parameters 5 and 6.

Although Figures 5.11 and 5.12 do not show the complete effect of Bayesian inference on the parameter distribution, the change in some of the parameter values is evident. For the prior distribution, the single parameter marginal distributions all have the shape of a normal distribution, given that the multivariate normal distribution is chosen as the form of the prior. The marginal posterior distributions, however, are estimated from a sample of points obtained at the end of the UQ algorithm, and thus are not given in the form of any standard type of distribution. The shapes of the marginal distributions vary by parameter. For some parameters, the value of maximum probability density changes significantly (e.g. Parameter 1) as shown in the shift in the probability density function (pdf). The change in this parameter's distribution is also shown by the shift in probability density in Figure 5.12A. In the joint marginal distribution 
for the two Henry's constant parameters (Figure 5.12B), the region of maximum probability density is also shifted and spans over a much smaller region.

The effect of Bayesian inference on the parameter distribution is visualized more clearly by propagating a sample of the posterior distribution through the thermodynamic model and comparing with the result obtained with the prior distribution using a sample of size 5000, which is used to evaluate the $\mathrm{CO}_{2}$ partial pressure as a function of temperature and $\mathrm{CO}_{2}$ loading, and the results are shown in Figure 5.13.

A

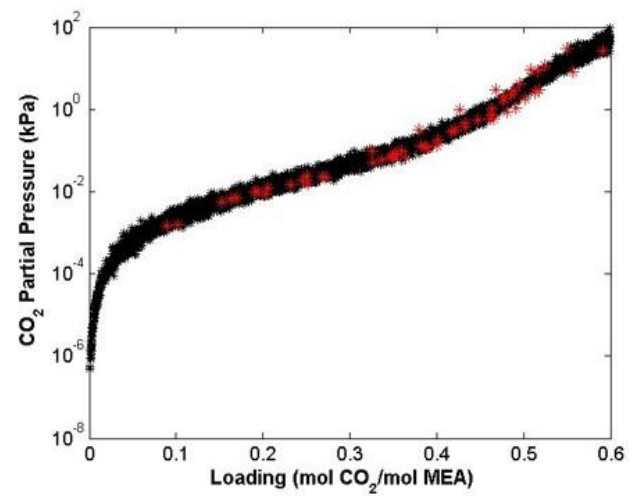

$\mathrm{C}$

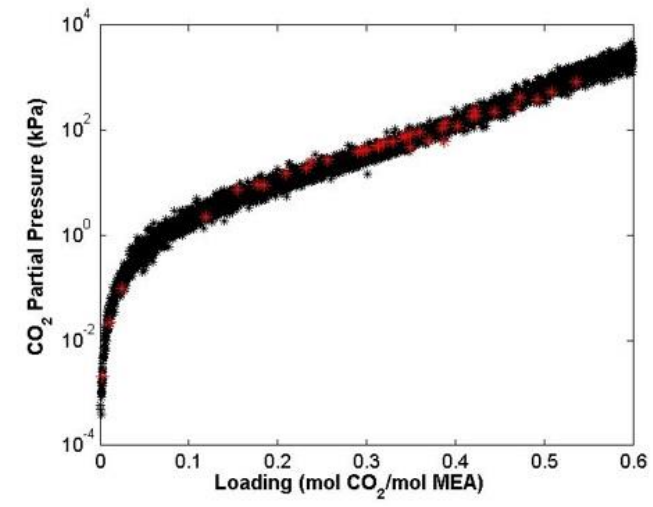

$\mathrm{B}$

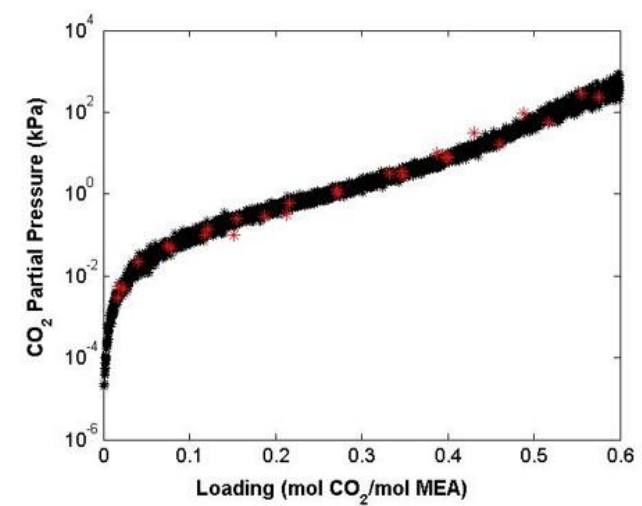

Figure 5-13. $\mathrm{CO}_{2}$ partial pressure prediction for stochastic model with a posterior parameter distribution with experimental data for $30 \mathrm{wt} \%$ MEA at temperatures of (a) $40^{\circ} \mathrm{C}$, (b) $80^{\circ} \mathrm{C}$, and (c) $120^{\circ} \mathrm{C}$.

The ranges of $\mathrm{CO}_{2}$ partial pressure predicted by the stochastic model with the posterior distribution are generally smaller than those predicted by the model with the prior distribution. This is expected, as relatively infeasible parameter space is removed from the distribution as a result of Bayesian inference. Moreover, the majority of the data fall within the range predicted by the model, which is a desirable outcome of Bayesian inference. These observations suggest 
that the posterior parameter distribution has been estimated accurately, even while using the response surface based Bayesian inference methodology.

\subsection{Case Studies with MEA-based $\mathrm{CO}_{2}$ Capture Process}

In order to determine the effect of process model uncertainty on the uncertainty in the performance of the solvent-based $\mathrm{CO}_{2}$ capture process, the parametric uncertainty is propagated through a sample process model. The sample process model is created by taking the model given by Plaza (2012) and replacing the viscosity, molar volume, surface tension, thermodynamic, and reaction kinetics models with those described in this work. The process is modeled in Aspen Plus V8.4, in which the absorber and stripper columns are modeled as rate-based towers using the RadFrac unit operation. The thermodynamic model is incorporated through the built-in ELECNRTL physical property method, and the reaction kinetics model is inserted into the simulation through a FORTRAN user model as discussed earlier. Because this analysis was performed separately for the three standalone models and the thermodynamic framework, the results are presented separately in the following subsections.

\subsubsection{Case Study with Viscosity, Molar Volume, and Surface Tension Models}

For the process model used in this case study, the only modifications made to the Plaza (2012) model are the replacement of the original viscosity, molar volume, and surface tension models with those developed in this work. The thermodynamic and reaction kinetics models developed in this work are not implemented in this process model as they had not been completed at the time that this analysis was performed.

For the model parameters determined to be important for UQ in this work, a sample size of 200 is drawn from the pdfs derived from Bayesian inference for all three standalone models. All parameters included in the deterministic models but not in the stochastic models are treated as constants. Absorber and regenerator (stripper) models are considered separately, and one operating condition is examined for each. For the absorber simulation, the output variable of interest is $\mathrm{CO}_{2}$ capture percentage, calculated as: 


$$
\% \mathrm{CO}_{2, \text { cap }}=\left(\frac{\dot{m}_{\mathrm{CO}_{2}, \mathrm{FG} G_{\text {in }}}-\dot{m}_{\mathrm{CO}_{2}, \mathrm{FG}} \text { out }}{\dot{m}_{\mathrm{CO}_{2}, \mathrm{FG} G_{\text {in }}}}\right) * 100 \%
$$

where $\dot{m}_{\mathrm{CO}_{2}, F G_{\text {in }}}$ and $\dot{m}_{\mathrm{CO}_{2}, F G_{\text {out }}}$ are the mass flow rates of $\mathrm{CO}_{2}$ in the absorber inlet and outlet gas streams, respectively. Essential input variables for the absorber simulation are given in Table 5.4 .

Table 5-4. Input variables for absorber simulation in case study with viscosity, molar volume, and surface tension parameters

\begin{tabular}{cc}
\hline Lean Solvent Flowrate $(\mathrm{kg} / \mathrm{hr})$ & 3000 \\
L/G (inlet liquid:gas mass ratio) & 4.42 \\
Inlet Lean Solvent Temperature $\left({ }^{\circ} \mathrm{C}\right)$ & 40 \\
Inlet Lean Solvent Pressure $(\mathrm{kPa})$ & 101.3 \\
Inlet Lean Solvent Loading $\left(\mathrm{mol} \mathrm{CO}_{2} / \mathrm{MEA}\right)$ & 0.35 \\
Inlet Lean Solvent $\mathrm{W}_{\mathrm{MEA}}(\%)$ & 35.4 \\
\hline
\end{tabular}

The effect of parametric uncertainty for the three property models on the uncertainty of the absorber model performance is estimated by simulating the model highlighted in Table 5.4 a total of 200 times, each with a different observation of the parameter values. The results of this procedure are given Table 5.5. The output variables considered are the percentage of $\mathrm{CO}_{2}$ capture and the values of the physical properties in the inlet lean solvent stream to the absorber. The uncertainty in the $\mathrm{CO}_{2}$ capture percentage that results from the propagation of the prior and posterior distributions through the process model is given in the form of estimated pdfs in Figure 5.14 . 
Table 5-5. Results of propagating prior and posterior distributions of viscosity, molar volume, and surface tension parameters though absorber simulation

\begin{tabular}{ccccccc}
\hline & \multicolumn{3}{c}{ Prior Distributions } & & \multicolumn{2}{c}{ Posterior Distributions } \\
\cline { 2 - 7 } & Range & Average & Standard & Range & Average & Standard \\
Deviation & & & 83.97 & 0.19 \\
\hline $\mathrm{CO}_{2, \mathrm{cap}}$ & $82.97-85.09$ & 84.05 & 0.46 & $83.53-84.48$ & 2.89 & 0.16 \\
$\mu_{\text {lean }}(\mathrm{mPa}-\mathrm{s})$ & $1.95-5.11$ & 2.95 & 0.70 & $2.53-3.31$ & $1.048-1.106$ & 0.012 \\
$\rho_{\text {lean }}\left(\mathrm{g} / \mathrm{cm}^{3}\right)$ & $1.052-1.113$ & 1.083 & 0.012 & $0.051-0.0776$ & 0.0664 & 0.0048 \\
$\sigma_{\text {lean }}(\mathrm{N} / \mathrm{m})$ & $0.0553-0.0786$ & 0.0670 & 0.0051 & & & \\
\hline
\end{tabular}




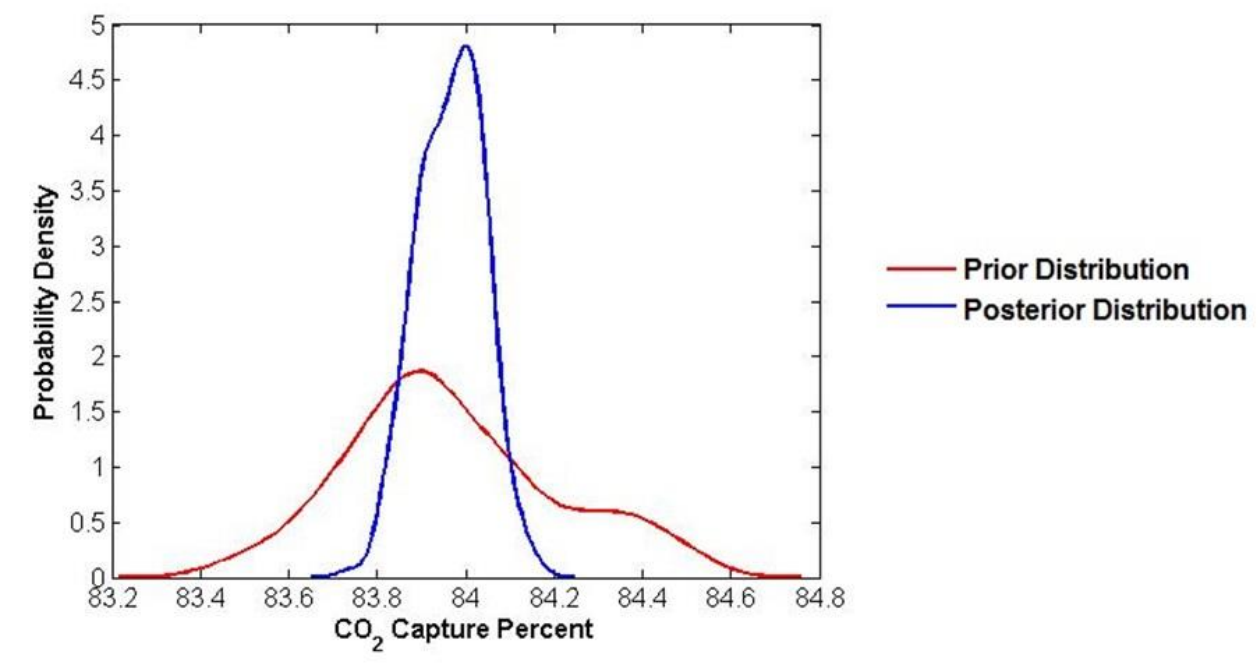

Figure 5-14. Estimated pdfs for absorber $\mathrm{CO}_{2}$ capture percentage resulting from propagating parametric uncertainty in viscosity, molar volume, and surface tension models through process model

As expected, the predicted range of $\mathrm{CO}_{2}$ capture percentage, as well as the ranges of the physical property values in the inlet solvent stream, decreases as a result of updating the parameter distribution through UQ. Moreover, a sensitivity analysis is also performed in which the correlation of the physical property values in the inlet solvent stream and the $\mathrm{CO}_{2}$ capture percentage of the absorber column is analyzed for each model in order to provide insight into the relative importance of the individual properties in determining the absorber efficiency. Figure 5.15 shows the individual relationships between the values of viscosity, density, and surface tension in the lean solvent stream and the calculated values of the coefficient of determination for each. The prior distributions are propagated through the model in this analysis, although similar trends would be expected if the posterior distributions were used instead. 
A

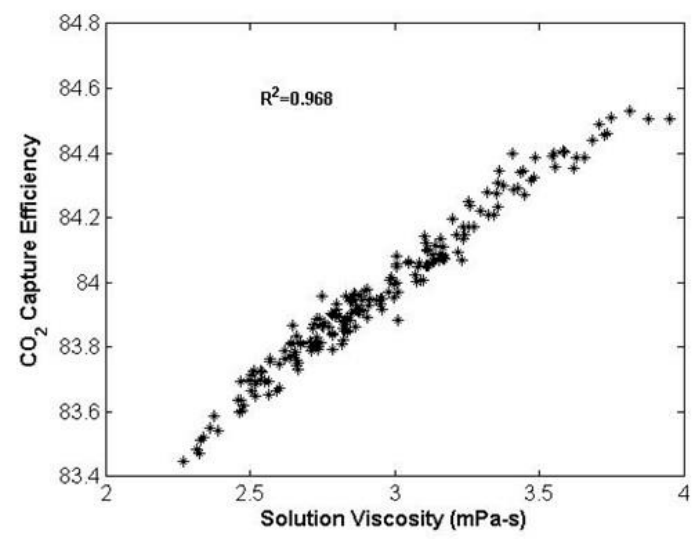

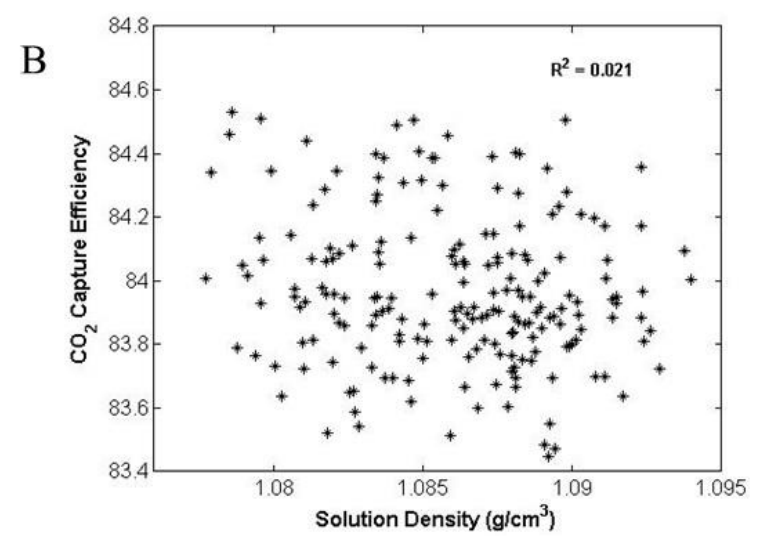

C

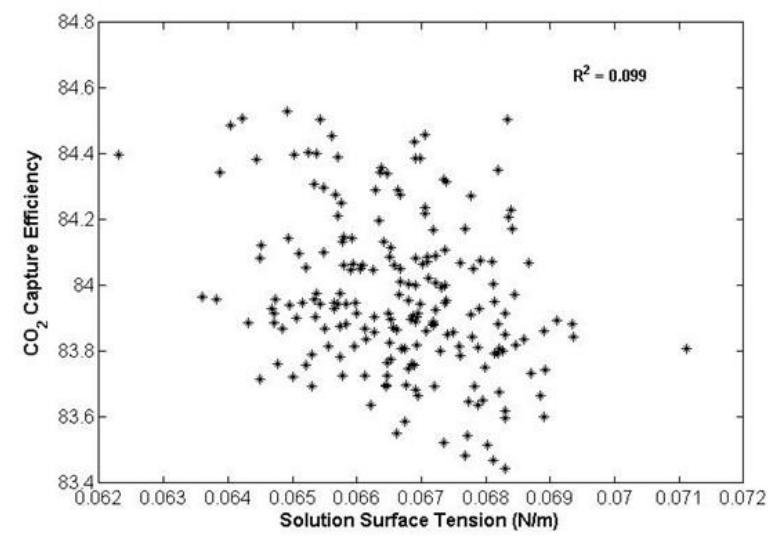

Figure 5-15. Correlations between (A) viscosity, (B) density, and (C) surface tension in the lean solvent stream and $\mathrm{CO}_{2}$ capture percentage of the absorber column. Variation in physical properties attributed to propagating prior parameter distributions through the process model for all three physical properties.

The variation in the $\mathrm{CO}_{2}$ capture percentage caused by simultaneously propagating parameter distributions from all of the physical property models mentioned here is strongly correlated with the variation in the viscosity in the inlet stream, especially in comparison with the density and surface tension.

For the stripper simulation, the reboiler duty $(\dot{Q})$ required to reduce the lean solvent loading to a given value $\left(\alpha_{\text {lean }}=0.3 \mathrm{~mol} \mathrm{CO} / \mathrm{MEA}\right)$ is the major output variable of interest, and the physical properties of the inlet rich solvent are also reported. The simulation input variables are given in Table 5.6, and the distributions of the output variables are described in Table 5.7. 
Table 5-6. Input variables for stripper simulation in case study with viscosity, molar volume, and surface tension parameters

\begin{tabular}{cc}
\hline Inlet Rich Solvent Mass Flowrate $(\mathrm{kg} / \mathrm{h})$ & 3100 \\
Inlet Rich Solvent Temperature $\left({ }^{\circ} \mathrm{C}\right)$ & 106.5 \\
Inlet Rich Solvent Pressure $(\mathrm{kPa})$ & 230.4 \\
Inlet Rich Solvent Loading $\left(\mathrm{mol} \mathrm{CO}_{2} / \mathrm{MEA}\right)$ & 0.5 \\
Inlet Rich Solvent $\mathrm{W}_{\mathrm{MEA}}(\%)$ & 35.4 \\
Outlet Lean Solvent Loading $(\mathrm{mol} \mathrm{CO} / \mathrm{MEA})$ & 0.3 \\
\hline
\end{tabular}


Table 5-7. Resulting of propagating prior and posterior distributions of viscosity, molar volume, and surface tension parameters through stripper column

\begin{tabular}{|c|c|c|c|c|c|c|}
\hline & \multicolumn{3}{|c|}{ Prior Distributions } & \multicolumn{3}{|c|}{ Posterior Distributions } \\
\hline & Range & Average & Standard & Range & Average & Standard \\
\hline & & & Deviation & & & Deviation \\
\hline$\dot{Q}(\mathrm{~kW})$ & $141.3335-141.3389$ & 141.3366 & 0.0013 & $141.3363-141.3378$ & 141.3370 & 0.0003 \\
\hline$\mu_{\text {rich }}(\mathrm{mPa}-\mathrm{s})$ & $0.69-1.91$ & 1.12 & 0.27 & $0.87-1.18$ & 1.03 & 0.07 \\
\hline$\rho_{\text {rich }}\left(\mathrm{g} / \mathrm{cm}^{3}\right)$ & $0.958-1.034$ & 0.997 & 0.015 & $1.000-1.029$ & 1.011 & 0.007 \\
\hline$\sigma_{\text {rich }}(\mathrm{N} / \mathrm{m})$ & 0.0451-0.0809 & 0.0631 & 0.0076 & $0.0544-0.0706$ & 0.0629 & 0.0039 \\
\hline
\end{tabular}


As shown in Table 5.7, the uncertainty in the reboiler duty that results from propagating the parametric uncertainty through the stripper model is negligible, regardless of whether the prior or posterior distribution is used. A likely reason that the parametric uncertainty has less effect on the stripper model in comparison to the absorber model is that the value of viscosity is much lower in the stripper model due to higher operating temperature. It must be noted that all three models are extrapolated to the operating temperature of the regenerator, since no data for these properties are available in the open literature at this temperature range.

\subsubsection{Case Study with Thermodynamic Framework}

The process model used for this case study is similar to that used in the work described in Section 5.3.1, although the thermodynamic framework and reaction kinetics models developed for this work have been incorporated into the process model. As before, separate case studies are performed for the absorber and stripper columns. For the absorber, two cases are considered and the model variables for these cases are summarized in Table 5.8. All variables, with the exception of $\mathrm{CO}_{2}$ capture percentage, are input variables to the simulation.

Table 5-8. Input variables for absorber simulation in case study with thermodynamic parameters

\begin{tabular}{ccc}
\hline Model Variable & Case 1 & Case 2 \\
\hline Inlet Solvent Flowrate $(\mathrm{kg} / \mathrm{hr})$ & 3000 & 4000 \\
Inlet Gas Flowrate $(\mathrm{kg} / \mathrm{hr})$ & 680 & 680 \\
Inlet Solvent Temperature $\left({ }^{\circ} \mathrm{C}\right)$ & 40 & 40 \\
Inlet Solvent Pressure $(\mathrm{kPa})$ & 101.325 & 101.325 \\
Inlet Solvent MEA wt\% & 35.5 & 35.5 \\
Inlet Solvent Loading $(\mathrm{mol} \mathrm{CO} / \mathrm{MEA})$ & 0.35 & 0.35 \\
$\mathrm{CO}_{2}$ Capture Percentage & 76.76 & 90.05 \\
\hline
\end{tabular}

One thousand samples are randomly drawn from both the posterior and prior distributions and propagated through the simulations as summarized in Table 5.8. The $\mathrm{CO}_{2}$ capture percentage is calculated for each simulation, and the pdfs corresponding to the prior and posterior distributions for each case are shown in Figure 5.16. 
A

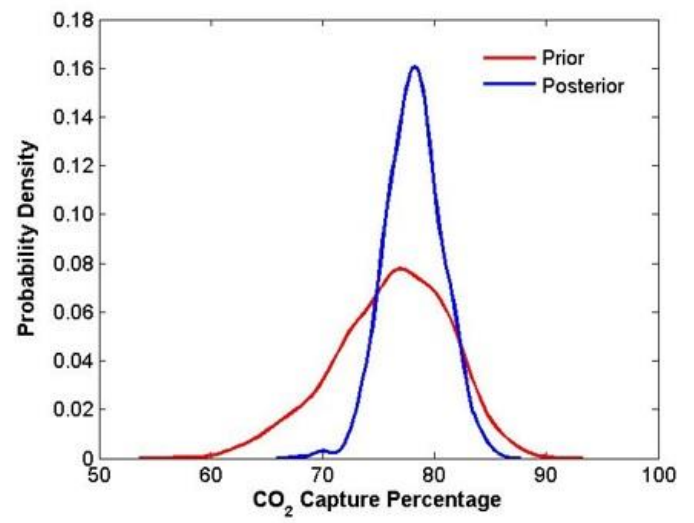

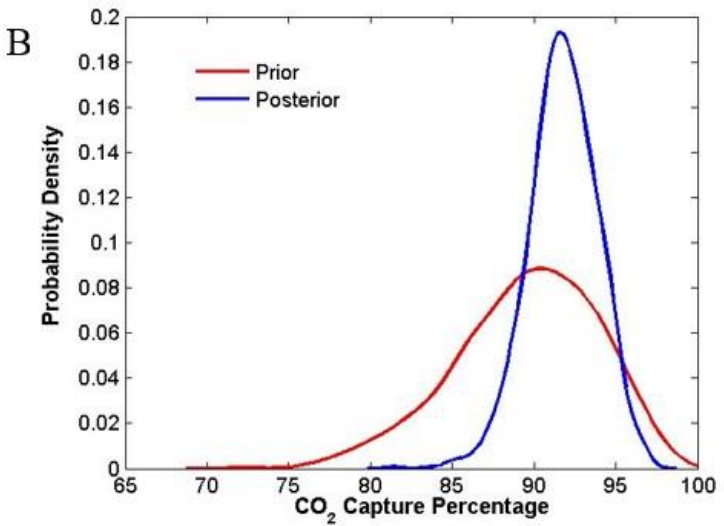

Figure 5-16. Pdfs for $\mathrm{CO} 2$ capture percentage for stochastic absorber models for (A) Case 1 and $(B)$ Case 2

In Figure 5.16, the sharper peaks corresponding to the posterior distribution for each of the two cases indicate that the most likely value of $\mathrm{CO}_{2}$ capture can be estimated more precisely as a result of performing the Bayesian inference methodology. Additionally, the range of $\mathrm{CO}_{2}$ capture predicted by the stochastic model with the posterior distribution is smaller compared to that predicted by the model with the prior distribution.

A similar analysis is performed for the stripper. Model variables for two cases are shown in Table 5.9. For this simulation, the output variable is the loading of the rich solvent at the stripper outlet. All other variables are input variables.

Table 5-9. Input variables for absorber simulation in case study with thermodynamic parameters

\begin{tabular}{ccc}
\hline Model Variable & Case 1 & Case 2 \\
\hline Inlet Solvent Flowrate $(\mathrm{kg} / \mathrm{hr})$ & 3100 & 3100 \\
Inlet Solvent Temperature $\left({ }^{\circ} \mathrm{C}\right)$ & 106.5 & 106.5 \\
Inlet Solvent Pressure $(\mathrm{kPa})$ & 230.4 & 230.4 \\
Inlet Solvent MEA wt\% & 35.5 & 35.5 \\
Inlet Solvent Loading $\left(\mathrm{mol} \mathrm{CO}_{2} / \mathrm{MEA}\right)$ & 0.5 & 0.3 \\
Reboiler Duty $(\mathrm{kW})$ & 140 & 400 \\
Outlet Solvent Loading $(\mathrm{mol} \mathrm{CO} / \mathrm{MEA})$ & 0.2982 & 0.0907 \\
\hline
\end{tabular}

One thousand samples are randomly drawn from both the posterior and prior distributions and propagated through the simulations described in Table 5.9. The lean loading is calculated for 
each simulation, and the pdfs corresponding to the prior and posterior distributions for each case are shown in Figure 5.17.

A

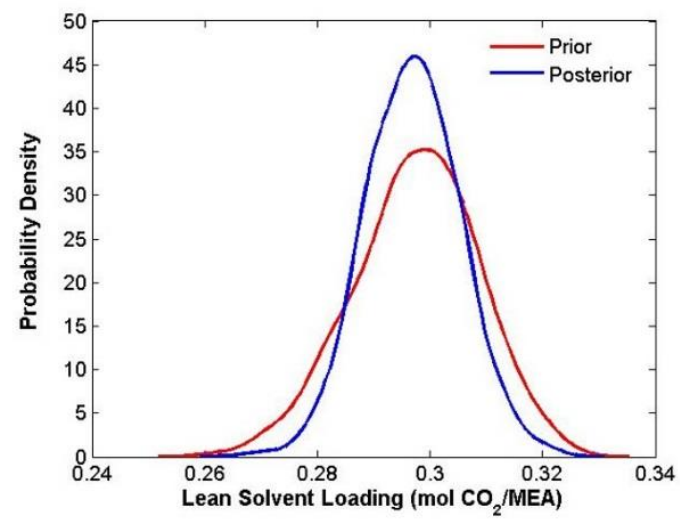

$\mathrm{B}$

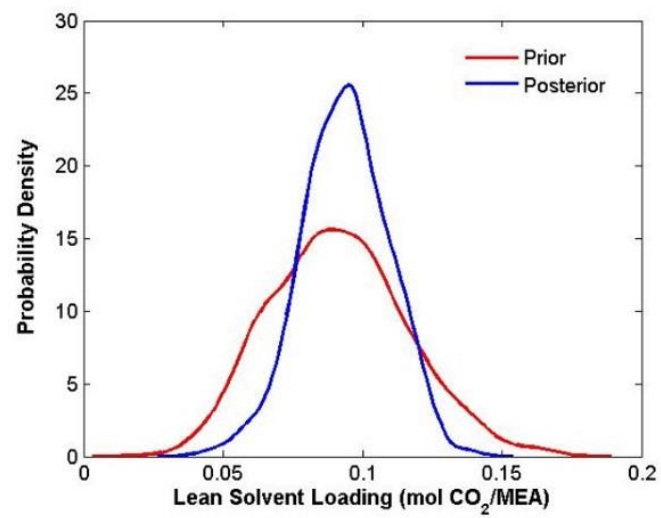

Figure 5-17. Pdfs for predicted lean solvent loading for stochastic regenerator models for (A) Case 1 and (B) Case 2

The general effect of Bayesian inference on the rich loading prediction is that the interval of feasible values becomes smaller and the probability density of the most likely value, given by the peak of the distribution, becomes higher. This trend is more pronounced for the lower loading case given in Figure 5.17. In comparison to the uncertainty in the stochastic models for viscosity, molar volume, and surface tension of this system, the uncertainty in the thermodynamic framework is shown to have a relatively large effect on the performance of the process model. This implies that the solvent-based $\mathrm{CO}_{2}$ capture process is more sensitive to the thermodynamic framework in comparison to the other physical property models for the system.

In the following chapter, the parametric uncertainty of all physical property models, along with that of other process submodels, will be propagated through the model of the NCCC pilot plant, the major process model of interest for this work. This will allow for more rigorous sensitivity and uncertainty analysis than that given in the case studies presented here.

\subsection{Conclusions}

A generalized approach to quantifying the uncertainty of property models is presented here. The Bayesian inference methodology deployed for this purpose seeks to update prior beliefs of parametric uncertainties with due consideration of the experimental data. To reduce the 
computational cost, response surfaces are used as surrogate models for Bayesian inference using the MCMC method with Gibbs sampling. For downselecting the parameter space of the standalone models (viscosity, molar volume, surface tension), a sensitivity matrix approach based on computing analytical derivatives of physical properties with respect to parameters is developed. This approach is found to be effective for all models for which it is tested. For the thermodynamic framework, the AIC approach for parameter selection was found to be useful for reducing the parameter space of the model and improving the computational expense of the process. Parametric uncertainty for all of the physical property models considered in this work has been propagated through absorber and stripper column models to analyze the effect of the submodel uncertainty on the overall process uncertainty. Preliminary results have demonstrated that the thermodynamic framework uncertainty has much larger effect on the process model in comparison to the viscosity, molar volume, and surface tension uncertainty. The following chapter is focused on the development of the complete process model for the NCCC system, combining the property models developed in this work with mass transfer and hydraulic models developed in other work related to this process. A more rigorous analysis of propagating submodel uncertainty through the process model will be explored for that system. 


\section{Chapter 6 : Steady State Model Validation}

\subsection{Steady State Test Design (NCCC)}

The validation data used for the MEA model developed in this work were obtained from a test campaign at the PSTU at NCCC in the summer of 2014. Although this work is focused on validation with steady-state data, dynamic data were also collected during this testing period. A number of important inputs, including disturbances, and operating conditions were selected for the steady-state test cases, which include the following variables:

- Solvent flowrate

- Flue gas flowrate

- Flue gas composition

- Lean solvent loading

- Number of absorber beds

- Presence/absence of intercooling in absorber column

The PSTU at NCCC is a relatively large scale pilot plant compared to many of the others for which large amounts of operation data are available in the open literature, with a $\mathrm{CO}_{2}$ capacity of 10 ton/day and a maximum stripper reboiler duty of about $700 \mathrm{~kW}$ in the data set collected for this work. The absorber and stripper columns consist of three and two beds, respectively, with each bed containing approximately $6 \mathrm{~m}$ of MellapakPlus ${ }^{\mathrm{TM}} 252 \mathrm{Y}$ structured packing. The column diameter is approximately $0.64 \mathrm{~m}$ for the absorber and $0.59 \mathrm{~m}$ for the stripper. Multiple solvent inlets are present in the absorber column so that the number of beds may be varied between 1-3, and intercoolers are present between the absorber beds.

A set of 31 test runs was proposed using a test matrix approach, in which variables are varied simultaneously in order to cover a large range of operating conditions, in order to obtain a final data set that is suitable for a rigorous model validation. Figure 6.1 shows an example of the test matrix approach in terms of simultaneous variation of flowrates of solvent, flue gas, and steam to the reboiler. 

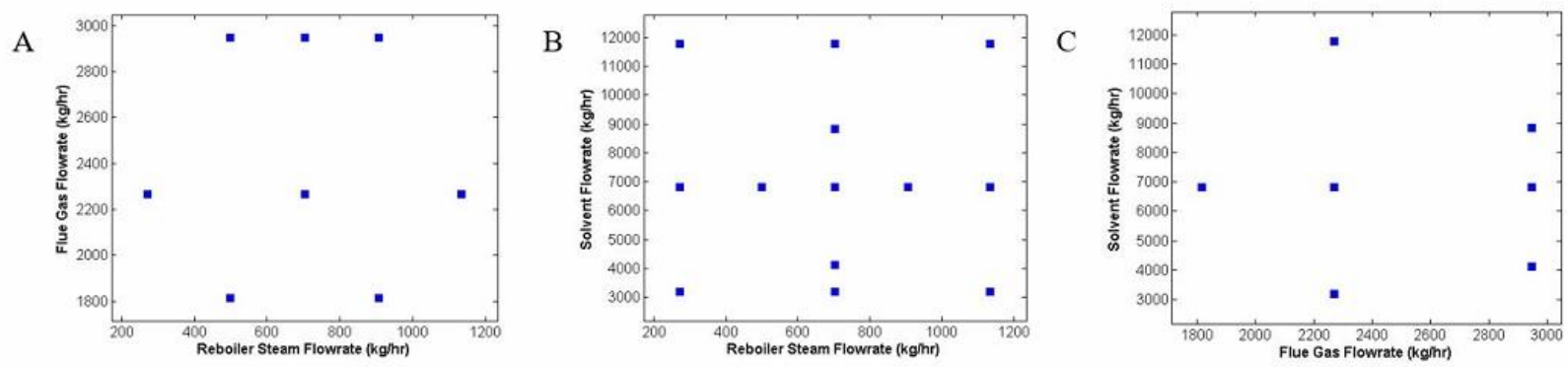

Figure 6-1. Simultaneous variation of flowrates in proposed test matrix for (A) flue gas flowrate vs. reboiler steam flowrate, (B) solvent flowrate vs. reboiler steam flowrate, and (C) solvent flowrate vs. flue gas flowrate.

A total of 23 steady-state runs were provided from the NCCC steady-state test. The first 17 test runs were conducted between $6 / 2 / 14$ and 6/12/14, and the test runs were then suspended due to process equipment failure. The runs were resumed on 8/20/2014, starting with dynamic test runs followed by six additional steady-state runs, and the test was concluded on 8/25/2014. The measured values of key operating variables are shown for each case in Tables 6.1-2. 
Table 6-1. Summary of NCCC steady state data (Absorber variables)

\begin{tabular}{|c|c|c|c|c|c|c|c|c|c|}
\hline $\begin{array}{c}\text { Case } \\
\text { No. }\end{array}$ & $\begin{array}{c}\text { Lean } \\
\text { Solvent } \\
\text { Flowrate } \\
(\mathrm{kg} / \mathrm{hr})\end{array}$ & $\begin{array}{c}\text { Lean } \\
\text { Solvent } \\
\text { Temperature } \\
\left({ }^{\circ} \mathrm{C}\right)\end{array}$ & $\begin{array}{c}\text { Lean Solvent } \\
\mathrm{CO}_{2} \text { Loading } \\
\left(\mathrm{mol} \mathrm{CO}_{2} / \mathrm{MEA}\right)\end{array}$ & $\begin{array}{l}\text { Nominal } \\
\text { Lean } \\
\text { Solvent } \\
\text { MEA } \\
\text { Weight } \\
\text { Fraction }\end{array}$ & $\begin{array}{c}\text { Gas } \\
\text { Flowrate } \\
(\mathrm{kg} / \mathrm{hr})\end{array}$ & $\begin{array}{c}\text { Gas } \\
\text { Temperature } \\
\left({ }^{\circ} \mathrm{C}\right)\end{array}$ & $\begin{array}{l}\mathrm{CO}_{2} \\
\mathrm{wt} \%\end{array}$ & $\begin{array}{c}\text { Column } \\
\text { Pressure } \\
(\mathrm{kPa})\end{array}$ & $\begin{array}{c}\text { Number of } \\
\text { Beds } \\
\text { (Intercoolers) }\end{array}$ \\
\hline K1 & 6804 & 40.97 & 0.145 & 0.298 & 2248 & 42.48 & 17.31 & 108.82 & $3(2)$ \\
\hline $\mathrm{K} 2$ & 11794 & 40.52 & 0.247 & 0.312 & 2243 & 44.94 & 17.18 & 107.06 & $3(2)$ \\
\hline $\mathrm{K} 3$ & 3175 & 45.68 & 0.091 & 0.295 & 2242 & 43.67 & 15.96 & 107.78 & $3(2)$ \\
\hline K4 & 3175 & 46.72 & 0.083 & 0.310 & 2243 & 44.73 & 17.24 & 107.65 & $3(2)$ \\
\hline K5 & 6804 & 41.57 & 0.108 & 0.306 & 2235 & 43.78 & 13.95 & 106.94 & $3(2)$ \\
\hline K6 & 6804 & 40.87 & 0.347 & 0.307 & 2237 & 42.18 & 13.81 & 107.10 & $3(2)$ \\
\hline K7 & 11791 & 40.62 & 0.399 & 0.288 & 2233 & 44.72 & 13.95 & 107.30 & $3(2)$ \\
\hline K8 & 11643 & 40.57 & 0.154 & 0.285 & 2237 & 42.47 & 13.93 & 107.26 & $3(2)$ \\
\hline K9 & 3175 & 42.66 & 0.239 & 0.311 & 2232 & 44.87 & 14.01 & 107.49 & $3(2)$ \\
\hline K10 & 3175 & 48.59 & 0.062 & 0.297 & 2237 & 42.15 & 13.90 & 107.71 & $3(2)$ \\
\hline K11 & 6804 & 40.83 & 0.161 & 0.293 & 2237 & 44.40 & 15.15 & 107.20 & $3(2)$ \\
\hline K12 & 6804 & 40.92 & 0.160 & 0.293 & 2231 & 43.24 & 12.55 & 106.37 & $3(2)$ \\
\hline K13 & 6804 & 41.93 & 0.164 & 0.303 & 2238 & 42.09 & 14.14 & 107.56 & $3(0)$ \\
\hline K14 & 8845 & 40.41 & 0.224 & 0.303 & 2895 & 47.67 & 13.76 & 107.86 & $3(2)$ \\
\hline K15 & 8845 & 40.60 & 0.224 & 0.313 & 2908 & 42.83 & 13.74 & 108.08 & $3(2)$ \\
\hline K16 & 4128 & 44.45 & 0.124 & 0.329 & 2903 & 45.42 & 14.00 & 108.70 & $3(2)$ \\
\hline K17 & 6804 & 42.03 & 0.168 & 0.307 & 2240 & 41.03 & 13.89 & 108.12 & $2(0)$ \\
\hline K18 & 6804 & 42.24 & 0.141 & 0.302 & 2271 & 46.07 & 15.46 & 109.18 & $1(0)$ \\
\hline K19 & 11793 & 40.90 & 0.184 & 0.278 & 1440 & 46.18 & 16.65 & 108.02 & $1(0)$ \\
\hline K20 & 3175 & 45.30 & 0.075 & 0.276 & 1324 & 46.09 & 16.63 & 108.12 & $1(0)$ \\
\hline $\mathrm{K} 21$ & 3175 & 45.18 & 0.074 & 0.271 & 1366 & 46.11 & 15.46 & 107.76 & $2(0)$ \\
\hline $\mathrm{K} 22$ & 6804 & 41.24 & 0.130 & 0.281 & 1379 & 46.06 & 16.53 & 107.51 & $2(1)$ \\
\hline $\mathrm{K} 23$ & 6804 & 41.23 & 0.138 & 0.281 & 1418 & 46.11 & 15.39 & 107.37 & $2(1)$ \\
\hline
\end{tabular}


Table 6-2. Summary of NCCC steady state data (Stripper variables)

\begin{tabular}{|c|c|c|c|c|c|c|c|}
\hline $\begin{array}{c}\text { Case } \\
\text { No. }\end{array}$ & $\begin{array}{c}\text { Rich } \\
\text { Solvent } \\
\text { Flowrate } \\
(\mathrm{kg} / \mathrm{hr}) \\
\end{array}$ & $\begin{array}{c}\text { Inlet } \\
\text { Solvent } \\
\text { Temperature } \\
\left({ }^{\circ} \mathrm{C}\right) \\
\end{array}$ & $\begin{array}{c}\text { Outlet } \\
\text { Solvent } \\
\text { Temperature } \\
\left({ }^{\circ} \mathrm{C}\right)\end{array}$ & $\begin{array}{c}\text { Rich } \\
\text { Loading } \\
\text { (mol } \\
\left.\mathrm{CO}_{2} / \mathrm{MEA}\right) \\
\end{array}$ & $\begin{array}{c}\text { Rich } \\
\text { MEA } \\
\text { weight } \\
\text { fraction }\end{array}$ & $\begin{array}{l}\text { Operating } \\
\text { Pressure } \\
(\mathrm{kPa})\end{array}$ & $\begin{array}{c}\text { Reboiler } \\
\text { Duty } \\
(\mathrm{kW})\end{array}$ \\
\hline K1 & 7242 & 104.8 & 120.18 & 0.384 & 0.300 & 183.87 & 431 \\
\hline $\mathrm{K} 2$ & 12284 & 104.8 & 117.43 & 0.385 & 0.314 & 182.06 & 430 \\
\hline K3 & 3335 & 97.4 & 122.15 & 0.475 & 0.313 & 184.36 & 427 \\
\hline K4 & 3343 & 97.6 & 122.53 & 0.470 & 0.328 & 184.15 & 427 \\
\hline $\mathrm{K} 5$ & 7212 & 109.0 & 121.68 & 0.295 & 0.308 & 183.43 & 673 \\
\hline K6 & 7063 & 95.7 & 110.21 & 0.469 & 0.309 & 179.88 & 173 \\
\hline $\mathrm{K} 7$ & 12092 & 93.2 & 103.76 & 0.471 & 0.289 & 184.41 & 170 \\
\hline K8 & 12043 & 110.1 & 120.35 & 0.275 & 0.289 & 183.45 & 677 \\
\hline K9 & 3337 & 98.4 & 117.69 & 0.474 & 0.318 & 182.84 & 166 \\
\hline K10 & 3358 & 94.5 & 124.00 & 0.477 & 0.309 & 182.67 & 671 \\
\hline K11 & 7241 & 107.4 & 119.88 & 0.378 & 0.294 & 182.67 & 425 \\
\hline K12 & 7173 & 109.2 & 120.56 & 0.341 & 0.294 & 183.81 & 422 \\
\hline K13 & 7078 & 107.6 & 120.65 & 0.360 & 0.307 & 183.19 & 419 \\
\hline K14 & 9393 & 104.8 & 119.35 & 0.420 & 0.302 & 184.02 & 420 \\
\hline K15 & 9349 & 103.5 & 119.32 & 0.413 & 0.313 & 182.00 & 420 \\
\hline K16 & 4347 & 98.6 & 122.94 & 0.476 & 0.349 & 181.86 & 419 \\
\hline K17 & 7051 & 107.7 & 120.49 & 0.354 & 0.314 & 181.94 & 418 \\
\hline K18 & 7099 & 105.9 & 120.38 & 0.349 & 0.304 & 184.06 & 425 \\
\hline K19 & 12161 & 107.9 & 118.00 & 0.276 & 0.281 & 183.76 & 427 \\
\hline K20 & 3369 & 99.7 & 120.39 & 0.393 & 0.279 & 183.56 & 438 \\
\hline K21 & 3370 & 99.7 & 120.36 & 0.385 & 0.272 & 184.36 & 437 \\
\hline K22 & 7161 & 108.3 & 119.16 & 0.291 & 0.271 & 183.13 & 427 \\
\hline K23 & 7146 & 108.4 & 199.32 & 0.283 & 0.282 & 181.80 & 427 \\
\hline
\end{tabular}

\subsection{Deterministic Model Results}

For each case described in Tables 6.1-2, the process model is evaluated twice. In the first simulation, the flowrates and compositions of the lean solvent and flue gas streams are set based on the information in Table 6.1, while the corresponding variables for the rich solvent that enters the stripper column are inherited from the results of the absorber simulation. The second simulation involves only the stripper column, and the rich solvent flowrate and composition are set according to the data values shown in Table 6.2. It should be noted that no model parameters are manipulated to improve the fit of the process model to the pilot plant data in this work. 
Table 6.3 shows a comparison of the model predictions of the $\mathrm{CO}_{2}$ capture percentage with the data values, which have been calculated from the liquid and gas-side mass balances around the absorber column.

Table 6-3. Comparison of model predictions of $\mathrm{CO}_{2}$ capture percentage with data values calculated from the liquid and gas side mass balances

\begin{tabular}{cccc}
\hline Case No. & $\begin{array}{c}\mathrm{CO}_{2} \text { Capture } \\
\text { Percentage (Data- } \\
\text { Liquid Side) }\end{array}$ & $\begin{array}{c}\mathrm{CO}_{2} \text { Capture } \\
\text { Percentage (Data-Gas }\end{array}$ & $\begin{array}{c}\mathrm{CO}_{2} \text { Capture } \\
\text { Percentage (Model } \\
\text { Prediction) }\end{array}$ \\
\hline K1 & 89.45 & 99.91 & 99.96 \\
K2 & 93.26 & 99.49 & 99.84 \\
K3 & 72.90 & 83.57 & 77.33 \\
K4 & 70.86 & 78.10 & 76.50 \\
K5 & 90.69 & 99.53 & 99.98 \\
K6 & 58.89 & 59.03 & 68.16 \\
K1 & 57.50 & 54.76 & 69.82 \\
K8 & 93.92 & 98.07 & 99.95 \\
K9 & 52.30 & 55.48 & 57.55 \\
K10 & 89.69 & 98.43 & 95.35 \\
K11 & 91.63 & 99.75 & 99.93 \\
K12 & 92.47 & 99.61 & 99.93 \\
K13 & 89.84 & 97.98 & 98.36 \\
K14 & 92.89 & 98.27 & 99.32 \\
K15 & 91.72 & 99.42 & 99.44 \\
K16 & 84.74 & 93.54 & 87.54 \\
K17 & 88.92 & 97.61 & 98.18 \\
K18 & 85.72 & 92.85 & 94.36 \\
K19 & 93.18 & 98.21 & 98.91 \\
K20 & 89.92 & 95.55 & 98.02 \\
K21 & 90.05 & 96.32 & 99.72 \\
K22 & 94.58 & 99.49 & 99.97 \\
K23 & 92.61 & 99.58 & 99.96 \\
\hline & & & \\
\hline
\end{tabular}

The difference between the liquid and gas-side values of $\mathrm{CO}_{2}$ capture for each case may be attributed to incomplete closure of the mass balance around the absorber column. The gas-side data are generally more consistent with the model predictions. A parity plot and residual plot of the model and gas-side data values of $\mathrm{CO}_{2}$ capture is shown in Figure 6.2. In the residual plot, the residual is represented as $y_{\text {model }}-y_{\text {data }}$, a convention followed throughout this work for calculations of residuals and error percentages. 

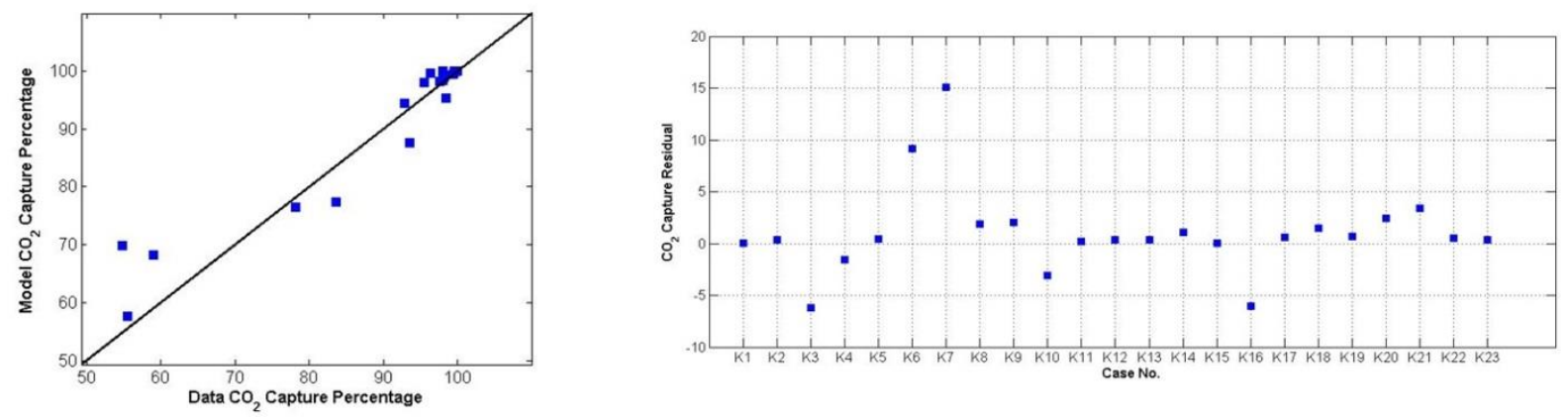

Figure 6-2. Parity plot and residual plot for gas side $\mathrm{CO}_{2}$ capture predicted by absorber model

The prediction of $\mathrm{CO}_{2}$ capture percentage is shown to be reasonably accurate, given that the discrepancy between model and data exceeds $5 \% \mathrm{CO}_{2}$ capture for only four cases, and $10 \%$ for only one. The overall correlation between model and data values of $\mathrm{CO}_{2}$ capture is $R^{2}=0.925$. Temperature profile data was also provided for the absorber and stripper columns during the test campaign at NCCC. The comparison of the model and experimental absorber liquid phase temperature profiles is given in Figures 6.3-4. 

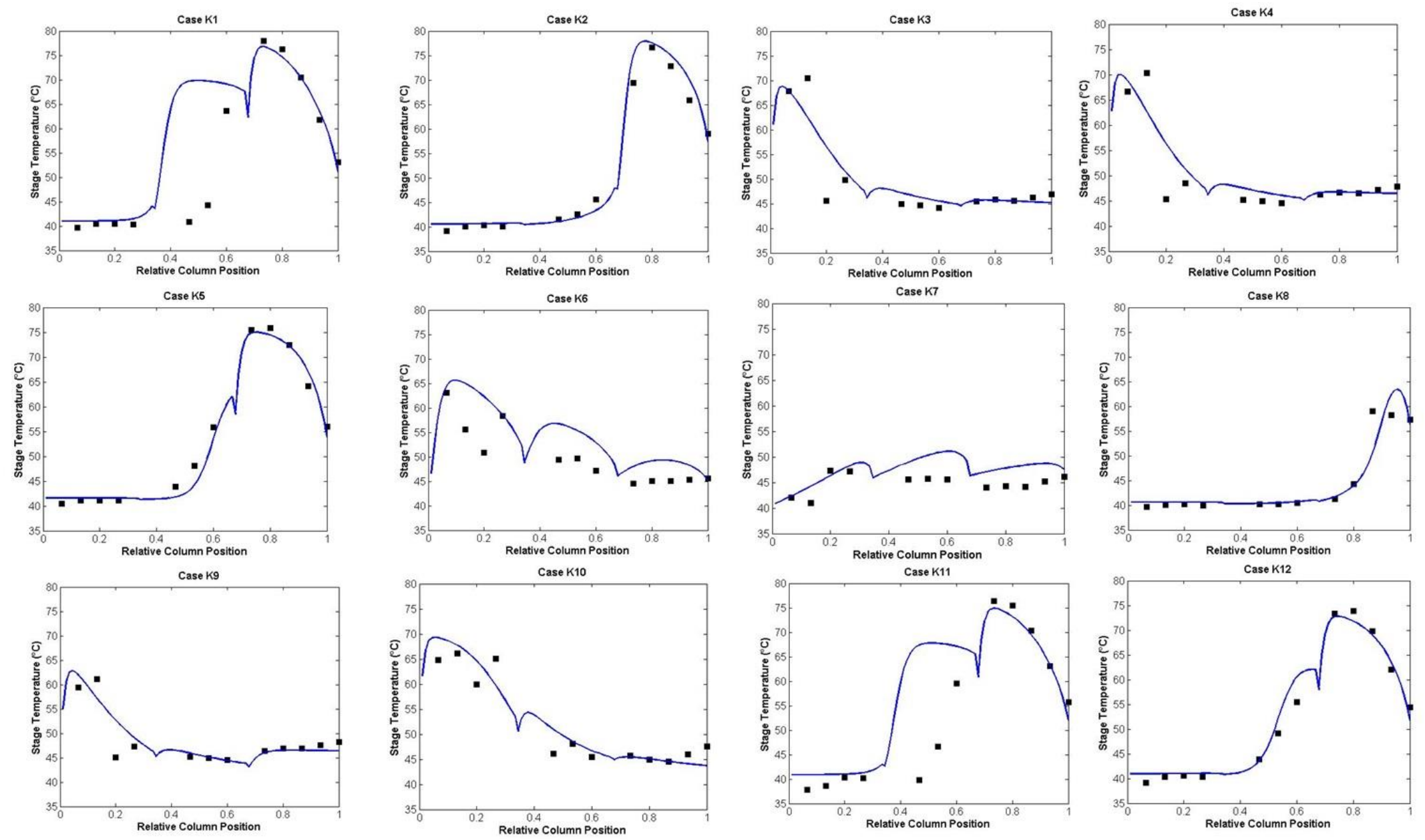

Figure 6-3. Comparison of model and data absorber temperature profiles for Cases K1-K12. Relative column positions of 0 and 1 represent top and bottom of column, respectively. 

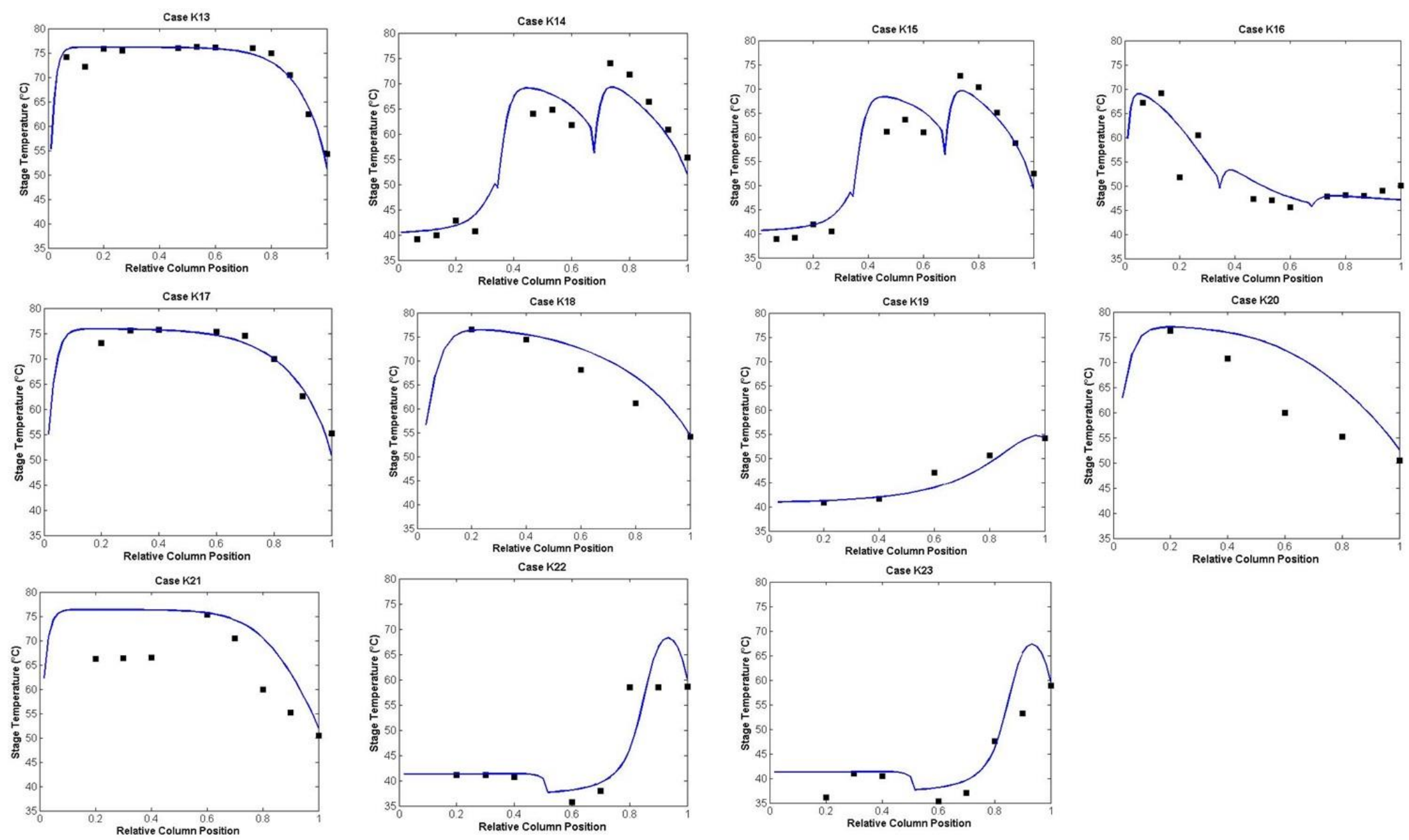

Figure 6-4. Comparison of model and data absorber temperature profiles for Cases K13-K23. Relative column positions of 0 and 1 represent top and bottom of column, respectively. 
As shown in the preceding figures, the temperature profile in the absorber may assume various shapes, depending on the L:G ratio, the inlet solvent loading, and the number of beds and intercoolers. The temperature bulges in the column, which occur as a result of the exothermic reaction between $\mathrm{CO}_{2}$ and $\mathrm{MEA}$, are generally located toward the top of the column during operation at low L:G ratio and toward the bottom for high L:G ratio. For most cases, the model is able to accurately predict the shapes of the various temperature profiles. To quantify the quality of the model fit of the temperature profile, an average temperature profile error is evaluated for each case, defined as:

$$
\widehat{T}_{\text {error }}=\frac{\sum_{i=1}^{n}\left|T_{i}-\bar{T}_{i}\right|}{n}
$$

where $T_{i}$ and $\bar{T}_{i}$ are the model and data values, respectively, of temperature at a given point in the column for which a measurement is given, and $n$ is the total number of such points. The value of for the absorber simulation for each case is shown in Figure 6.5.

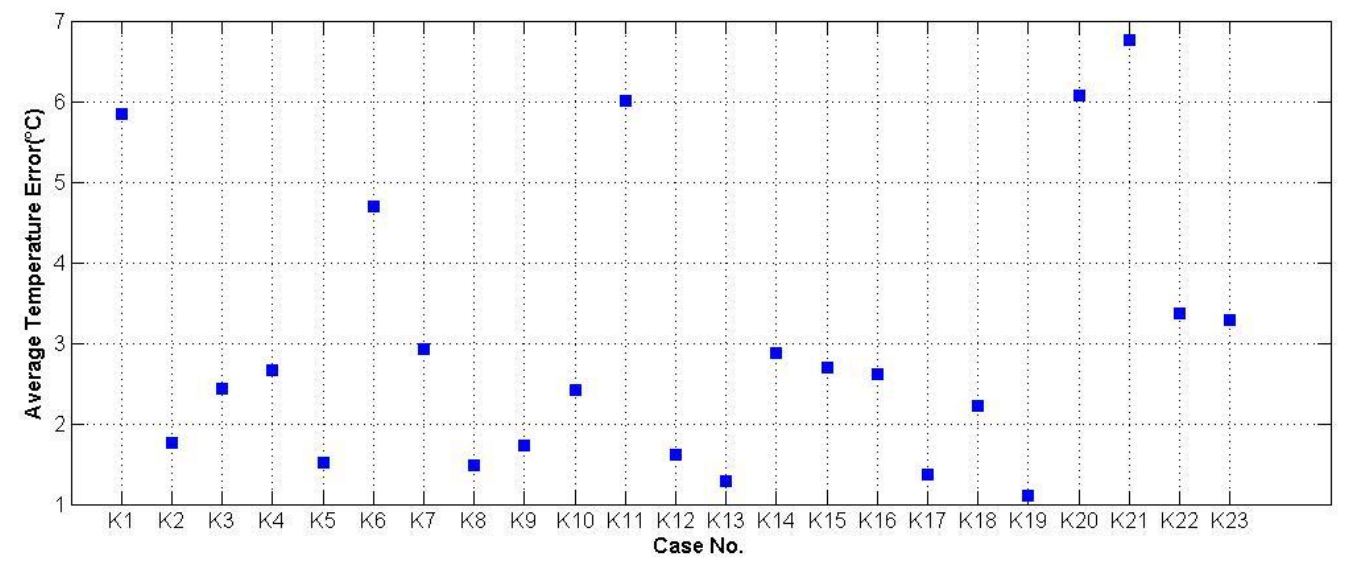

Figure 6-5. Values of temperature profile error (Eqn. 6.1) calculated for absorber column simulations

For the entire set of 23 cases, the average temperature error in the absorber column is $2.988 \pm$ $1.707^{\circ} \mathrm{C}$, and the value is below $3^{\circ} \mathrm{C}$ for most cases. For some of the cases for which this value is higher (e.g. K1 and K11), the large values may be attributed to a discrepancy in the location of a temperature bulge in the middle of the column, while the match of the temperature profile near the top and bottom of the column is quite accurate. The comparison of the experimental values and model predictions of the $\mathrm{CO}_{2}$ loading of the lean solvent from the regenerator outlet is given in Table 6.4 and Figure 6.6 for the combined simulation and the separate regenerator simulation. 
Table 6-4. Comparison of model predictions of $\mathrm{CO}_{2}$ loading in the outlet lean solvent from the regenerator

\begin{tabular}{cccc}
\hline Case No. & Data Value & $\begin{array}{c}\text { Model Value } \\
\text { (Regenerator Only) }\end{array}$ & $\begin{array}{c}\text { Model Value } \\
\text { (Complete System) }\end{array}$ \\
\hline K1 & 0.145 & 0.169 & 0.169 \\
K2 & 0.247 & 0.267 & 0.273 \\
K3 & 0.091 & 0.089 & 0.086 \\
K4 & 0.083 & 0.092 & 0.090 \\
K5 & 0.108 & 0.116 & 0.114 \\
K6 & 0.347 & 0.366 & 0.374 \\
K7 & 0.399 & 0.429 & 0.428 \\
K8 & 0.154 & 0.166 & 0.165 \\
K9 & 0.239 & 0.245 & 0.243 \\
K10 & 0.062 & 0.060 & 0.059 \\
K11 & 0.161 & 0.168 & 0.166 \\
K12 & 0.166 & 0.164 \\
K13 & 0.160 & 0.173 & 0.173 \\
K14 & 0.164 & 0.236 & 0.240 \\
K15 & 0.224 & 0.239 & 0.244 \\
K16 & 0.224 & 0.122 & 0.119 \\
K17 & 0.124 & 0.174 & 0.173 \\
K18 & 0.168 & 0.169 & 0.169 \\
K19 & 0.141 & 0.213 & 0.216 \\
K20 & 0.184 & 0.081 & 0.080 \\
K21 & 0.075 & 0.079 & 0.078 \\
K22 & 0.074 & 0.154 & 0.151 \\
K23 & 0.130 & 0.158 & 0.156 \\
\hline
\end{tabular}
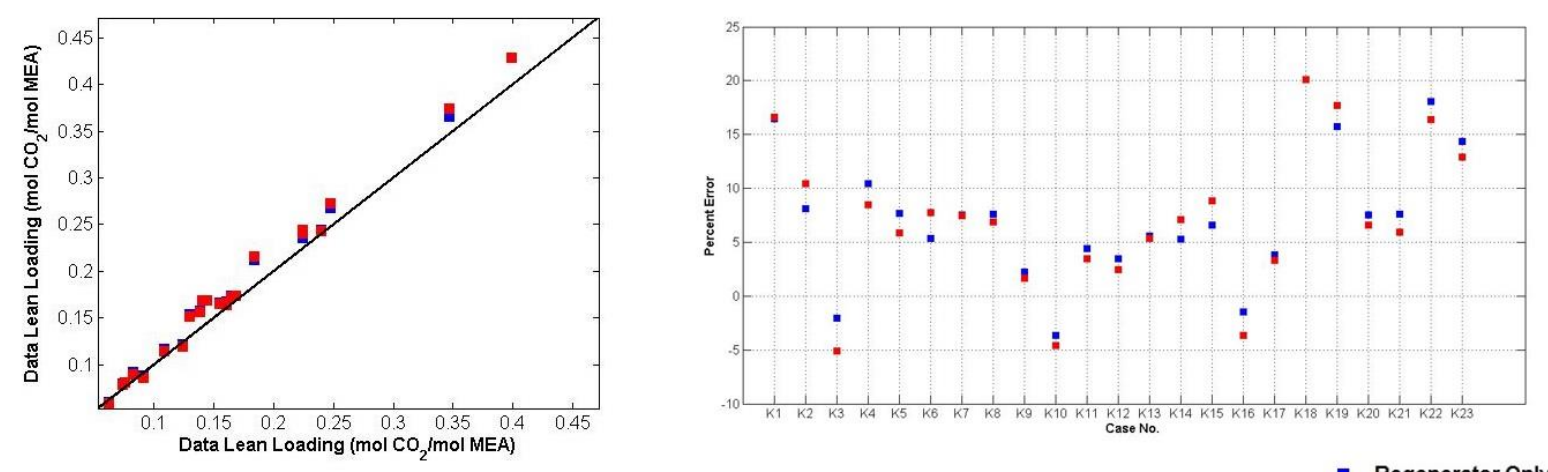

- Regenerator Only

Figure 6-6. Comparison of experimental data and model prediction of $\mathrm{CO}_{2}$ loading in the regenerator solvent outlet 
The fit of model and experimental lean loading is shown to be adequate, and the absolute value of the percent error does not exceed $20 \%$ for any case. The prediction of lean loading does not change appreciably with regard to whether the regenerator is simulated separately or as part of the overall process, and the correlation between model and data is approximately $R^{2}=0.991$ for both comparisons. The comparison of the model and data temperature profiles is shown in Figures 6.7-8. 

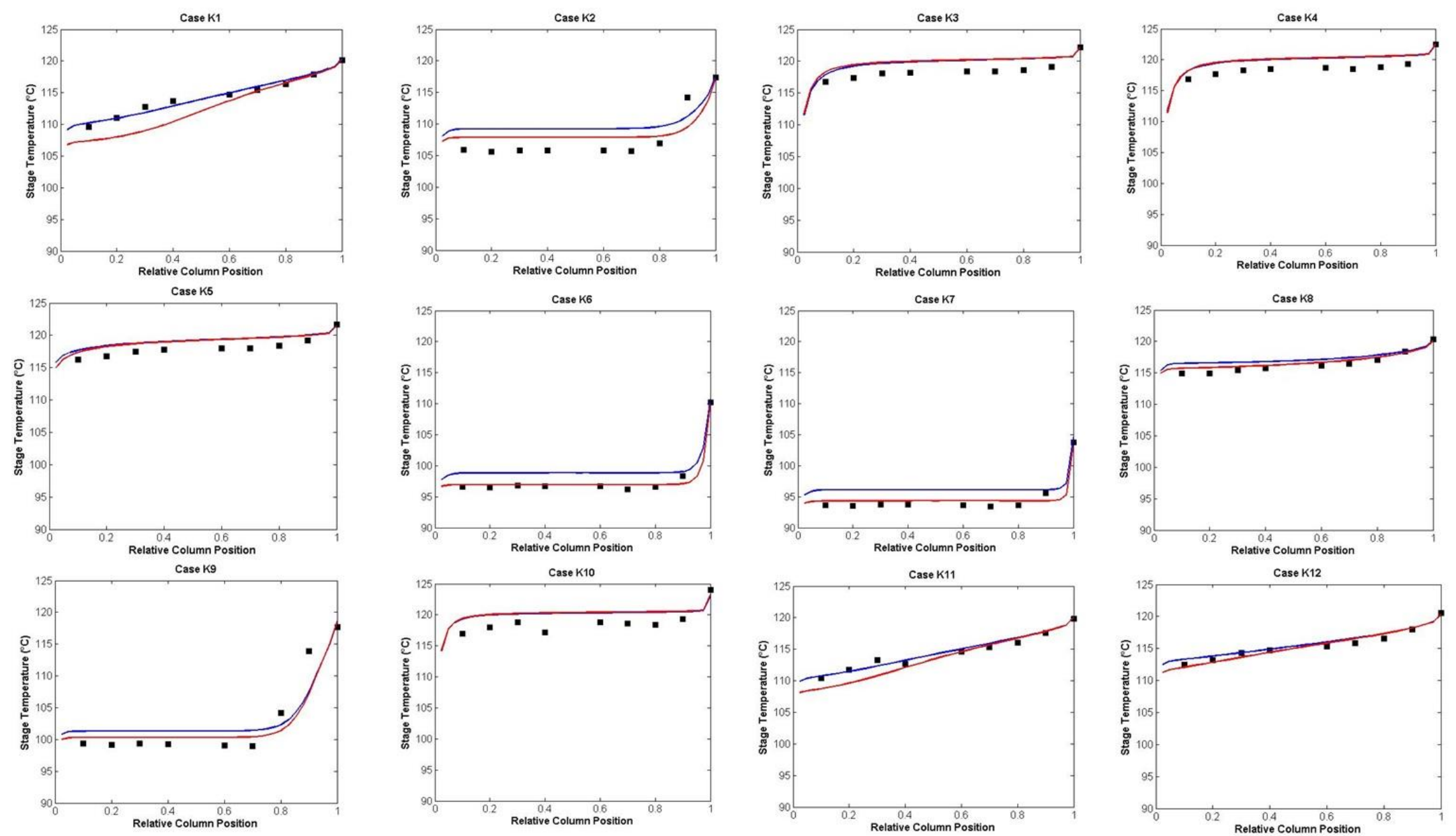

Figure 6-7. Comparison of model and data stripper temperature profiles for Cases K1-K12. Relative column positions of 0 and 1 represent top and bottom of column, respectively. Red and blue lines represent simulation results for complete process model and individual regenerator model, respectively. 

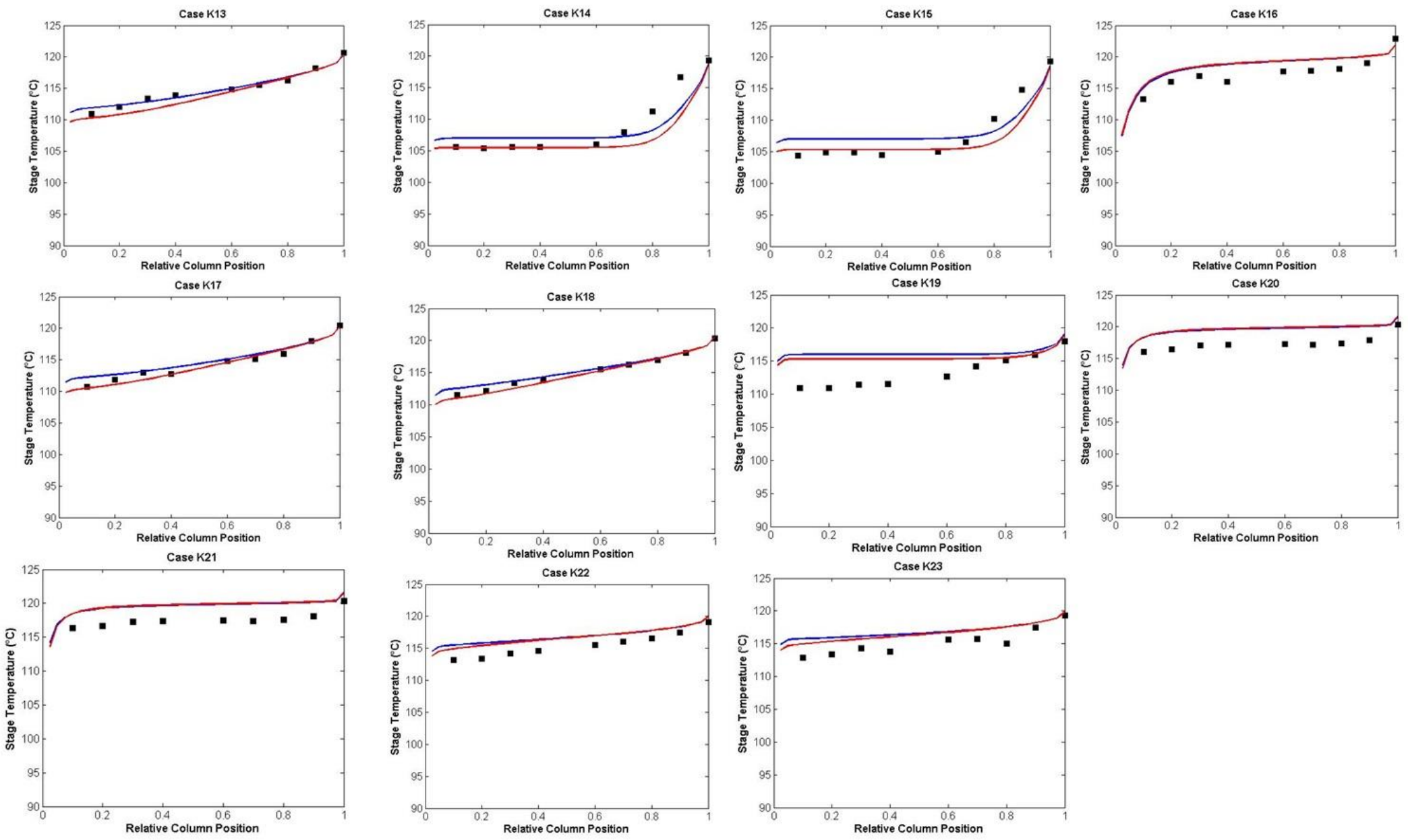

Figure 6-8. Comparison of model and data stripper temperature profiles for Cases K13-K23. Relative column positions of 0 and 1 represent top and bottom of column, respectively. Red and blue lines represent simulation results for complete process model and individual regenerator model, respectively. 
The shape of the temperature profile for the stripper is also dependent on the operating variables, notably the rich solvent flowrate and $\mathrm{CO}_{2}$ loading and the reboiler duty. The simulated temperature profiles match the experimental profiles accurately for most cases. For many cases, the fit is visibly improved when the entire system is simulated simultaneously, although there are some exceptions to this (e.g. Case K1). The temperature of the solvent exiting the column, given as the temperature value at a relative column position of 1 , is captured accurately by the model for nearly all cases, although less so for the later cases (K19-K23). Note that Cases K18-K23 were conducted separately, following the dynamic test runs, and there could be errors in the data not present for the first set of cases. The temperature profile error is calculated for the stripper column simulations and shown in Figure 6.9.

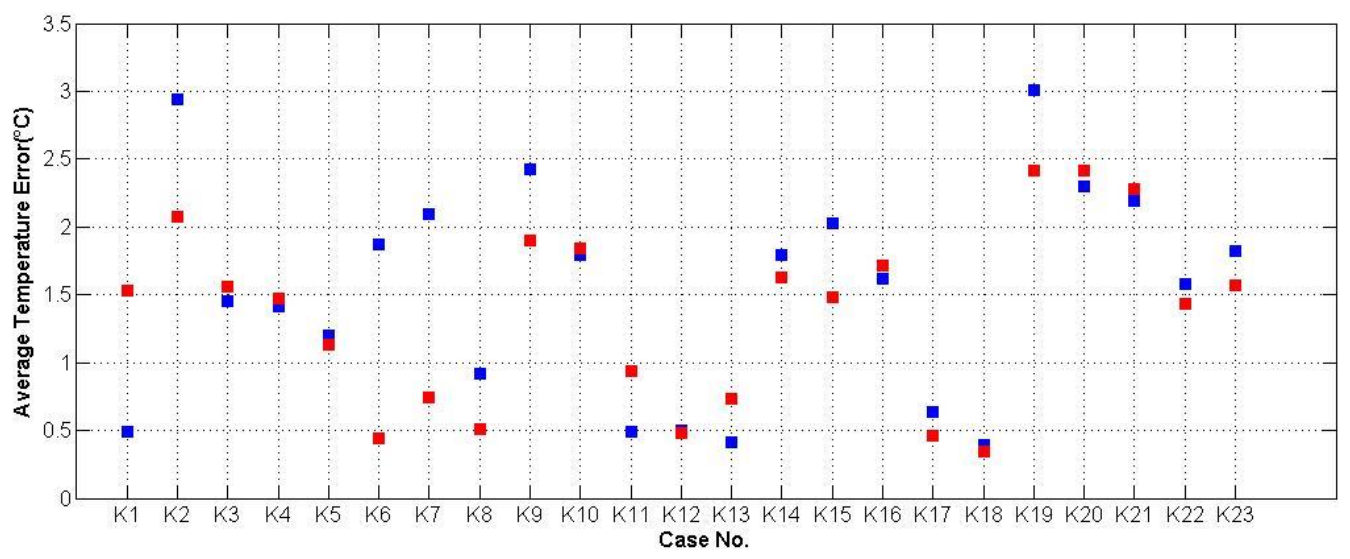

Figure 6-9. Values of temperature profile error (Eqn. 6.1) calculated for stripper column simulation. Blue squares represent case in which stripper column is simulated separately and red squares represent combined absorber/stripper simulation.

For the 23 cases as a whole, the average temperature error is $1.352 \pm 0.662$ for the complete system simulation and $1.537 \pm 0.797$ for the separate regenerator simulation. 


\subsection{Uncertainty Quantification}

\subsubsection{Physical Property Model Parameters}

As described in Chapter 5.3, work has been completed to demonstrate the effect of propagating parametric uncertainty from the physical property models through the overall process model on the process performance. A more rigorous analysis is performed here in which the parametric uncertainty of all property models is propagated simultaneously through the model in order to determine to which parameters, and which properties, the process model is sensitive.

This analysis makes use of a Sobol sensitivity analysis (Sobol, 1993), in which the relative contribution to the uncertainty of each parameter to the uncertainty of the process variables is estimated. In this analysis, a dependent variable that is calculated as a function of $k$ independent variables $\left(y=f\left(x_{1}, x_{2}, \ldots, x_{k}\right)\right)$ is considered. Sensitivity indices are calculated for the contributions of individual parameters $\left(S_{i}\right)$ and interactions among pairs $\left(S_{i j}\right)$ or groups $\left(S_{1,2, \ldots, k}\right)$ to the overall variability of the dependent variables. These terms satisfy the equation:

$$
\sum_{i=1}^{k} S_{i}+\sum_{1 \leq i<j \leq k} S_{i j}+\cdots+S_{1,2, \ldots, k}=1
$$

Since Eq. 6.2 includes $2^{k}-1$ terms, which becomes very large for the examples presented here, total Sobol indices $\left(S_{T i}\right)$ are presented in this work in lieu of the individual values. Each term $S_{T i}$ represents the total sensitivity of an individual parameter and its aggregate interactions of all orders. In this work, the output variables are represented as a function of the uncertain parameters for each base through response surface models built with MARS (Friedman, 1991), the same methodology used for creating the response surface models used for Bayesian inference. The response surface model methodology reduces the computational expense of this process by providing a surrogate model for calculating the process outputs for parameter realizations, reducing the required amount of process simulations.

Initially, only physical property model parameters are incorporated into this sensitivity analysis. As a review, the 22 parameters considered for UQ are summarized in Table 6.5. 
Table 6-5. List of physical property model parameters for which distribution are included in stochastic model

\begin{tabular}{|c|c|c|}
\hline Parameter No. & Parameter Name & Value \\
\hline \multicolumn{3}{|c|}{ Thermodynamic Model Parameters } \\
\hline 1 & DGAQFM (MEA+) & -190 \\
\hline 2 & DGAQFM (MEACOO-) & -492 \\
\hline 3 & DHAQFM (MEA+) & -330 \\
\hline 4 & DHAQFM (MEACOO-) & -691 \\
\hline 5 & HENRY/1 (MEA- $\left.\mathrm{H}_{2} \mathrm{O}\right)$ & 28.6 \\
\hline 6 & $\mathrm{HENRY} / 2\left(\mathrm{MEA}-\mathrm{H}_{2} \mathrm{O}\right)$ & -7610 \\
\hline 7 & NRTL/1 (MEA- $\left.\mathrm{H}_{2} \mathrm{O}\right)$ & 3.25 \\
\hline 8 & NRTL/1 ( $\left.\mathrm{H}_{2} \mathrm{O}-\mathrm{MEA}\right)$ & 4.34 \\
\hline 9 & NRTL/2 $\left(\mathrm{H}_{2} \mathrm{O}-\mathrm{MEA}\right)$ & -2200 \\
\hline \multicolumn{3}{|c|}{ Viscosity Model Parameters } \\
\hline 10 & $\mathrm{f}$ & 0.0103 \\
\hline 11 & $\mathrm{~g}$ & -2.39 \\
\hline \multicolumn{3}{|c|}{ Surface Tension Model Parameters } \\
\hline 12 & $\mathrm{a}$ & 2.46 \\
\hline 13 & $\mathrm{c}$ & 3.50 \\
\hline 14 & $\mathrm{~d}$ & -5.64 \\
\hline 15 & $\mathrm{e}$ & 10.2 \\
\hline 16 & $\mathrm{f}$ & 2.31 \\
\hline 17 & $\mathrm{~g}$ & 4.56 \\
\hline 18 & $\mathrm{j}$ & -12.0 \\
\hline \multicolumn{3}{|c|}{ Molar Volume Model Parameters } \\
\hline 19 & $\mathrm{a}$ & 10.2 \\
\hline 20 & $\mathrm{~b}$ & -2.26 \\
\hline 21 & $\mathrm{~d}$ & 207 \\
\hline 22 & $\mathrm{e}$ & -563 \\
\hline
\end{tabular}

The parameter definitions for the standalone models are given in Eqs. 3.1 (viscosity), 3.13 (molar volume), and 3.20-21 (surface tension). A sample of size 1000 is propagated through the process model, with the coupled absorber and stripper model, for three cases. For each observation from the sample, the $\mathrm{CO}_{2}$ capture percentage of the absorber and the lean loading at the stripper outlet are calculated. The estimated pdfs of the absorber $\mathrm{CO}_{2}$ capture and the outlet lean loading are given in Figures 6.10 and 6.11, respectively. 

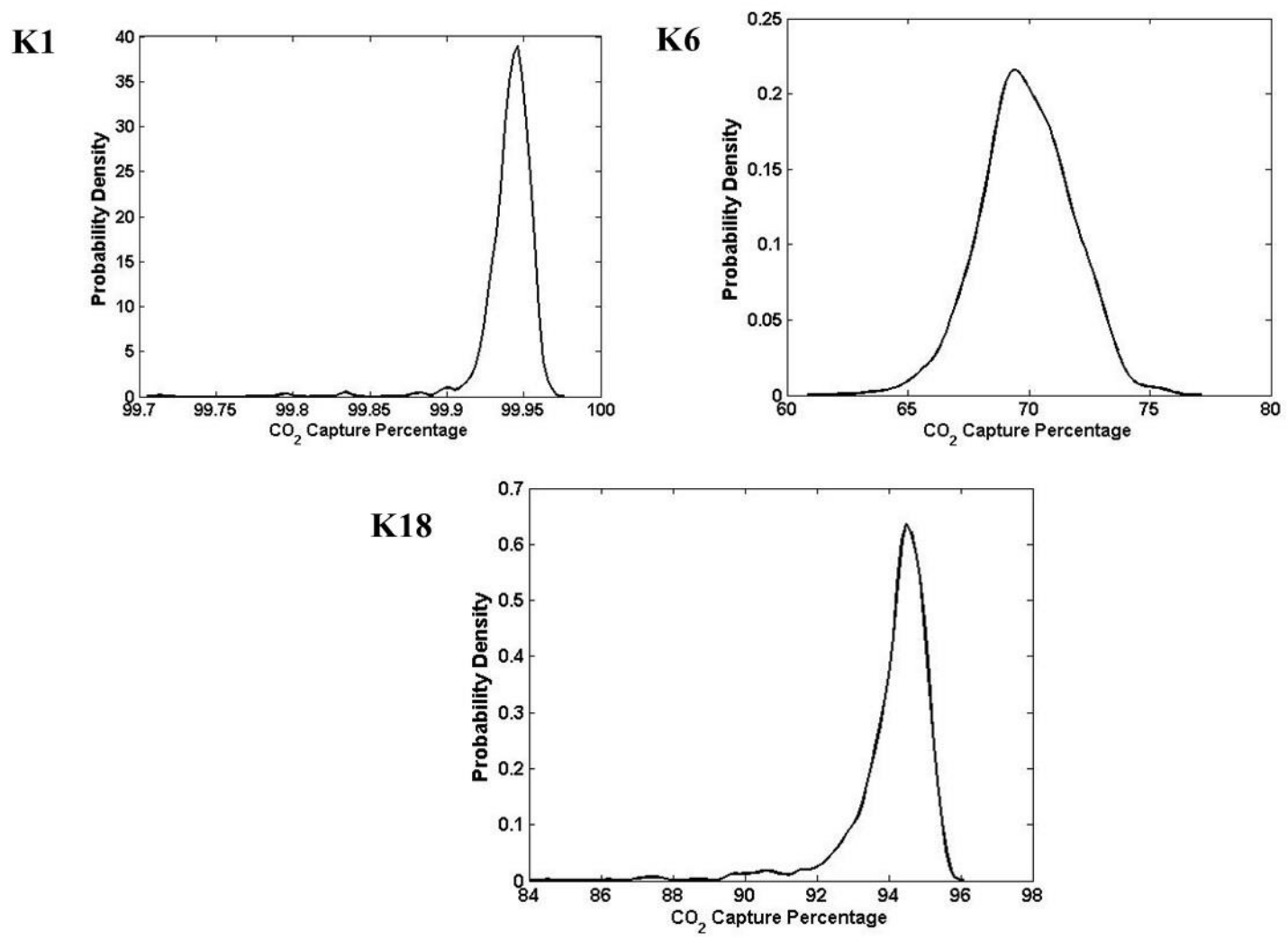

Figure 6-10. Estimated pdfs of $\mathrm{CO}_{2}$ capture percentage resulting from propagating physical property model parameters through process model 
K1

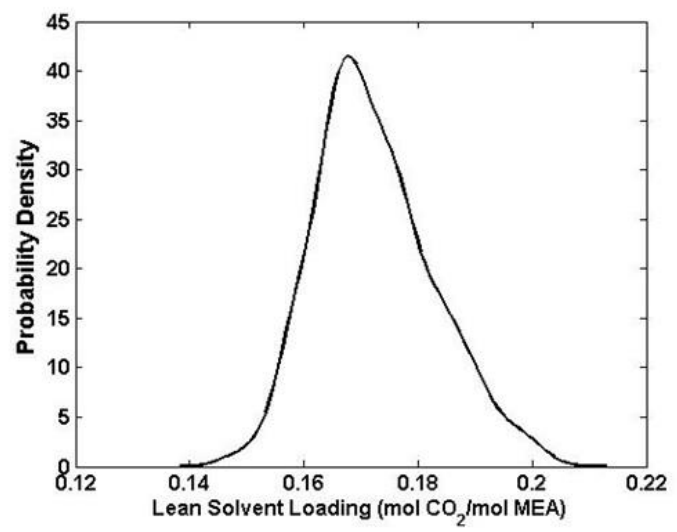

K6

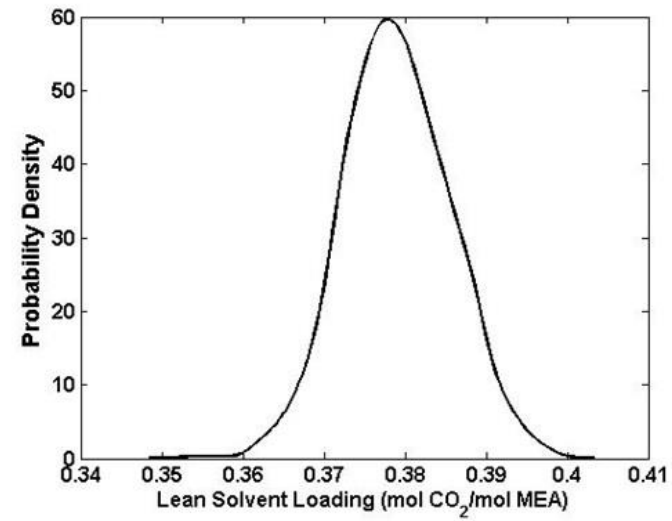

K18

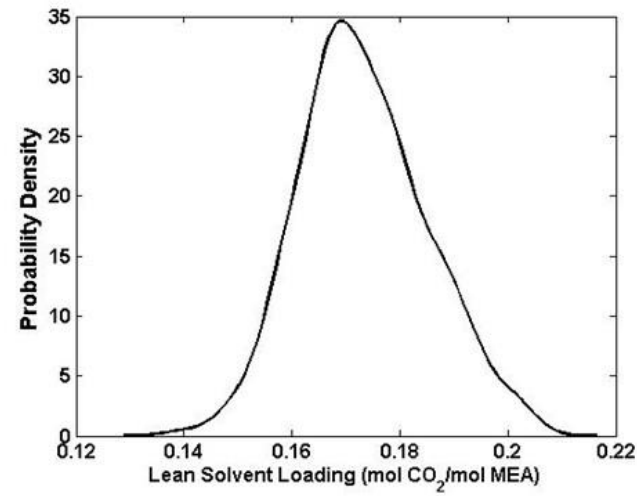

Figure 6-11.Estimated pdfs of output lean solvent loading resulting from propagating physical property model parameters through process model

The amount of uncertainty in the absorber efficiency resulting from propagating the physical property model parameters through the process model is shown to vary significantly based upon the process condition. For Case $\mathrm{K} 1$, in which the absorber is operating near complete $\mathrm{CO}_{2}$ capture, the uncertainty in $\mathrm{CO}_{2}$ capture is very small. For Case K6, for which the column operates in a reaction-limited regime and the $\mathrm{CO}_{2}$ capture percentage is lower, there is an approximate uncertainty range of $10 \%$, while the distribution is centered on the deterministic model value. Case K18 serves as an intermediate condition in which the absorber is slightly below complete $\mathrm{CO}_{2}$ capture, and the estimated uncertainty is a bit lower than in Case K6. For the stripper model, the uncertainty in the outlet lean loading is a bit more uniform for these three cases, with an uncertainty ranging approximately between $0.04-0.08 \mathrm{~mol} \mathrm{CO} / \mathrm{MEA}$. The total Sobol indices are calculated for the two process variables for the three cases and presented in Table 6.6. 
Table 6-6. Results of Sobol sensitivity analysis for NCCC absorber and stripper model for three cases

\begin{tabular}{|c|c|c|c|c|c|c|}
\hline \multirow[t]{2}{*}{ Parameter No. } & \multicolumn{3}{|c|}{$\mathrm{CO}_{2}$ Capture Percentage } & \multicolumn{3}{|c|}{ Lean Loading in Stripper Outlet } \\
\hline & K1 & K6 & K18 & K1 & K6 & K18 \\
\hline \multicolumn{7}{|c|}{ Thermodynamic Model Parameters } \\
\hline 1 & 0.0099 & 0.0477 & 0.0050 & 0.0274 & 0.0372 & 0.0268 \\
\hline 2 & 0.0147 & 0.0002 & 0.0063 & 0.0292 & 0.0166 & 0.0307 \\
\hline 3 & 0.0401 & 0.0132 & 0.0035 & 0.0303 & 0.0285 & 0.0346 \\
\hline 4 & 0.0251 & 0.0003 & 0.0042 & 0.0293 & 0.0135 & 0.0355 \\
\hline 5 & 0.2039 & 0.3023 & 0.1014 & 0.5065 & 0.4515 & 0.5062 \\
\hline 6 & 0.1538 & 0.2362 & 0.0717 & 0.3293 & 0.3204 & 0.3136 \\
\hline 7 & 0.0003 & 0.0038 & 0.0013 & 0.0003 & 0.0015 & 0.0002 \\
\hline 8 & 0.5214 & 0.2685 & 0.5823 & 0.0512 & 0.0961 & 0.0546 \\
\hline 9 & 0.4889 & 0.2714 & 0.5556 & 0.0465 & 0.0906 & 0.0478 \\
\hline \multicolumn{7}{|c|}{ Viscosity Model Parameters } \\
\hline 10 & 0 & 0 & 0.0031 & 0 & 0 & 0 \\
\hline 11 & 0 & 0 & 0.0027 & 0 & 0 & 0 \\
\hline \multicolumn{7}{|c|}{ Surface Tension Model Parameters } \\
\hline 12 & 0.0081 & 0 & 0 & 0 & 0 & 0 \\
\hline 13 & 0 & 0 & 0 & 0 & 0 & 0 \\
\hline 14 & 0.0165 & 0 & 0 & 0 & 0 & 0.0005 \\
\hline 15 & 0.0147 & 0 & 0 & 0 & 0 & 0 \\
\hline 16 & 0.0165 & 0 & 0 & 0 & 0 & 0 \\
\hline 17 & 0 & 0 & 0 & 0 & 0 & 0 \\
\hline 18 & 0 & 0 & 0 & 0 & 0 & 0 \\
\hline \multicolumn{7}{|c|}{ Molar Volume Model Parameters } \\
\hline 19 & 0.0034 & 0 & 0 & 0 & 0 & 0 \\
\hline 20 & 0 & 0 & 0 & 0 & 0 & 0 \\
\hline 21 & 0.0112 & 0 & 0 & 0 & 0 & 0 \\
\hline 22 & 0 & 0 & 0 & 0 & 0 & 0 \\
\hline
\end{tabular}

As shown in Table 6.6, most of the uncertainty in the process variables may be attributed to the thermodynamic model parameters, based on the value of the Sobol indices. With little exception, the total Sobol indices are calculated as 0 from this methodology, indicating that the parameter uncertainty has negligible effect on the calculation of the uncertainty in the process variable. For Case K1, some surface tension and molar volume parameters are reported to have discernable effect on the uncertainty of the $\mathrm{CO}_{2}$ capture percentage. However, this phenomenon may be attributed to computational error due to the extremely small overall uncertainty in the absorber model for this case. Based on this analysis, it is decided to use only the thermodynamic model parameter uncertainty to represent the property model uncertainty when propagating the 
submodel parametric uncertainty through the overall process model. This is described in the following section.

\subsubsection{Incorporation of Mass Transfer and Hydraulic Model Parameters}

In another project related to the development of this MEA model, mass transfer and hydraulics models are developed with UQ; details of these models are discussed in Chinen et al. (2017), and some summary is presented here. The parameters determined to contain considerable uncertainty are summarized in Tables 6.7-8 for the mass transfer and hydraulics models, respectively.

Table 6-7. Mass transfer model parameters considered for UQ

\begin{tabular}{ccc}
\hline Parameter No. & Parameter Name & Value \\
\hline 10 & ARVAL/2 & 1.42 \\
11 & DFACT/CO $_{2}$ & $4.56 \times 10^{-10}$ \\
\hline
\end{tabular}

Table 6-8. Hydraulics model parameters considered for UQ

\begin{tabular}{ccc}
\hline Parameter No. & Parameter Name & Value \\
\hline 12 & HURVAL/1 & 11.45 \\
13 & HURVAL/2 & 0.647 \\
\hline
\end{tabular}

In Tables 6.7-8, the parameters are assigned the names given to them in Aspen Plus ${ }^{\circledR}$. The parameters ARVAL and DFACT are associated with the interfacial area and liquid diffusivity models, respectively. While propagating the parametric uncertainty through the process model, the value of the interfacial area parameter is modified simultaneously for the absorber and stripper columns. HURVAL refers to liquid holdup model parameters. Note that the parameter numbers are assigned only for identification purposes and parameters 1-9 are still considered to be the thermodynamic model parameters as defined in Table 6.5. Estimated pdfs for the mass transfer and hydraulics model parameters are given in Figures 6.12-13. 

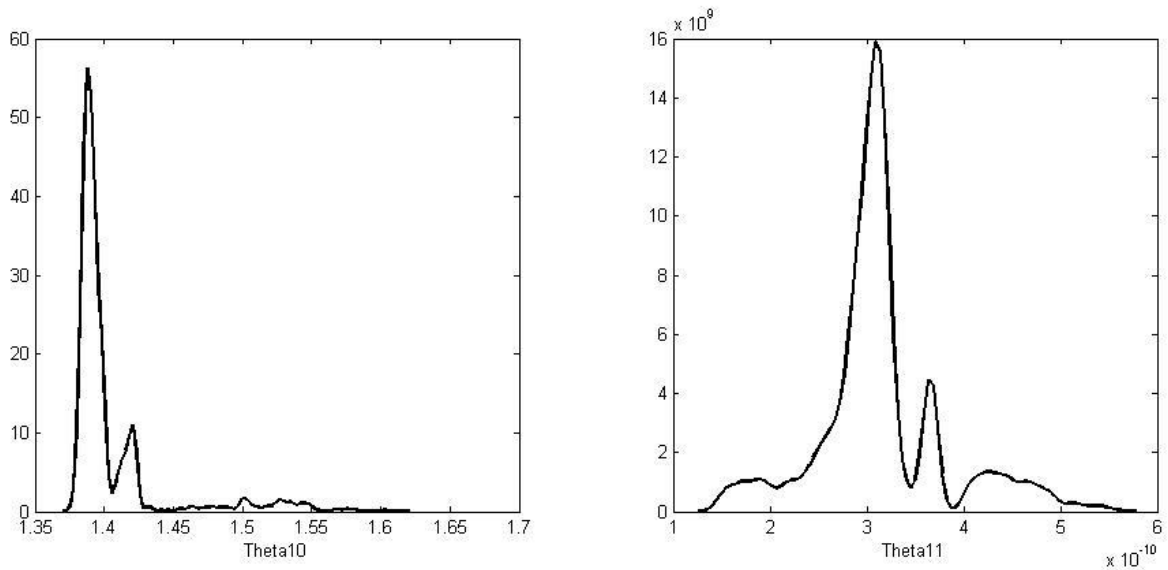

Figure 6-12. Estimated pdfs for single parameter marginal distributions of mass transfer model parameters considered for UQ
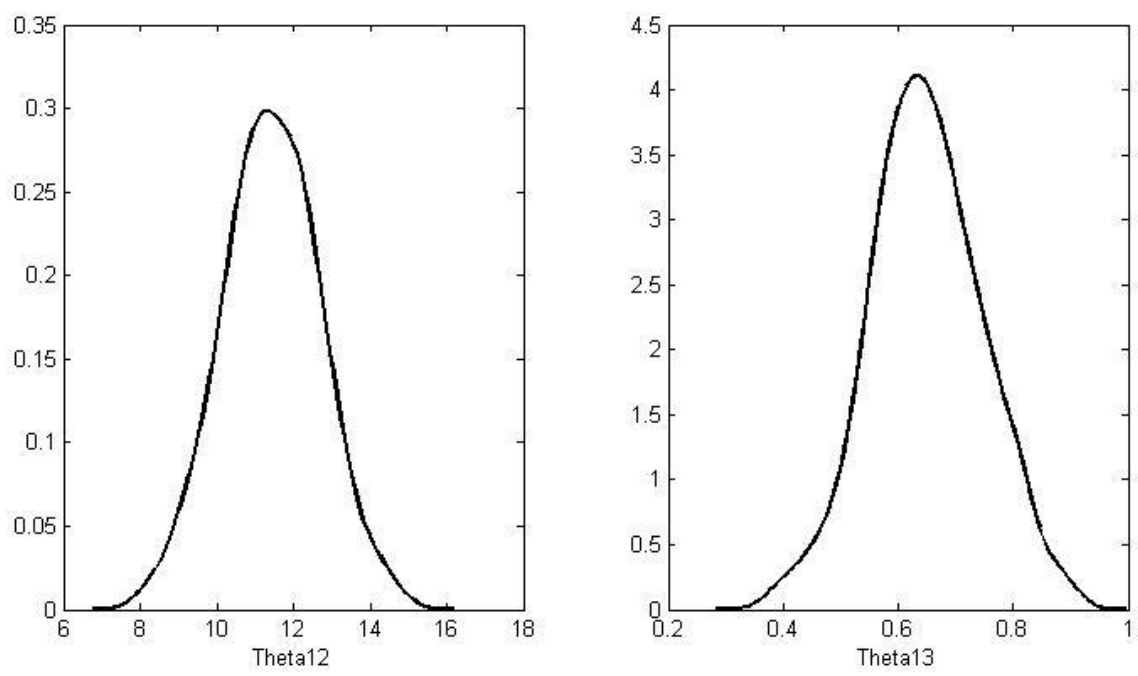

Figure 6-13. Estimated pdfs for single parameter marginal distributions of hydraulic model parameters considered for $\mathrm{UQ}$

A sample of 1000 is drawn from the distributions of the mass transfer and hydraulics parameters, concatenated with the sample of the same size from the posterior distribution of the thermodynamic model parameters considered in the previous section, and propagated through the process model. The estimated pdfs of $\mathrm{CO}_{2}$ capture and lean loading in the stripper outlet solvent are presented in Figure 6.14. 
K1

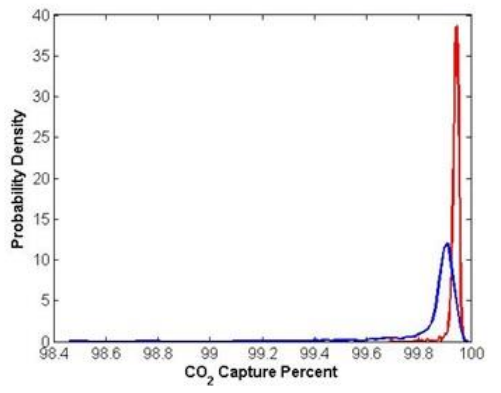

B

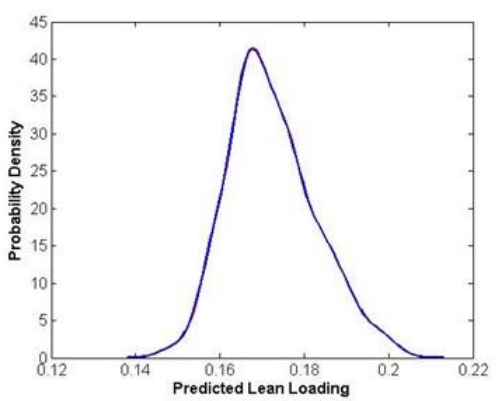

K6
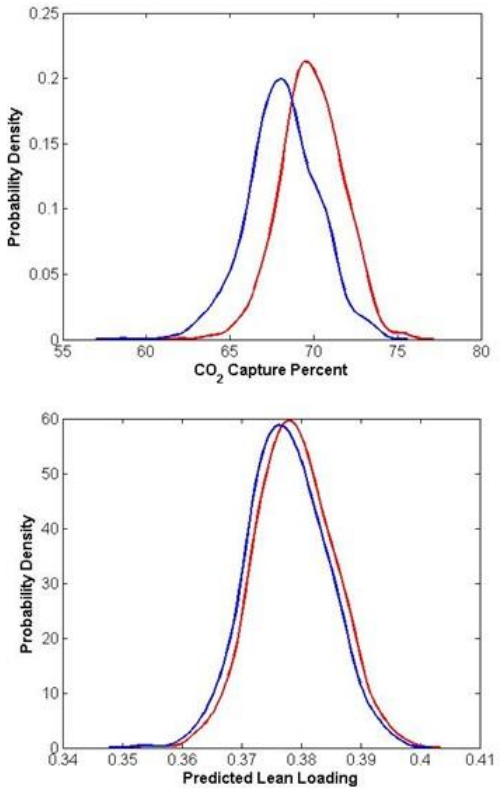

K18
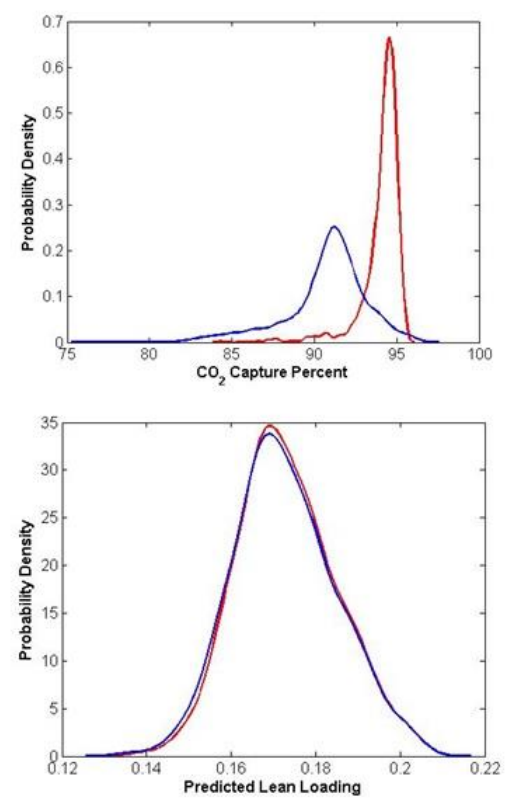

Figure 6-14. Estimated pdfs resulting from propagating parametric uncertainty through process model for three cases. Pdfs for $\mathrm{CO}_{2}$ capture percentage given in (A) and predicted lean loading in (B). Red lines represent case in which thermodynamic model parameter uncertainty is considered and blue lines represent case in which thermodynamic, mass transfer, and hydraulics model uncertainty is considered.

The results given in Figure 6.14 allow for comparison in the effect of thermodynamic and mass transfer model uncertainty separately. For all three cases, the model value of $\mathrm{CO}_{2}$ capture percentage is shifted slightly downward as a result of the inclusion of the mass transfer model uncertainty. As expected, the performance of the stripper column, which operates close to an equilibrium condition, remains relatively unaffected by the mass transfer model uncertainty. This is evident by the minimal change in the distributions of outlet lean loading for the three cases. The slightly perceptible change in the distribution estimated for Case K6, and Case K18 to a lesser extent, may be attributed to the change in the distributions of rich solvent flowrate and $\mathrm{CO}_{2}$ loading that result from the absorber model simulation. As the absorber model operates at a nearly complete $\mathrm{CO}_{2}$ capture regime for Case $\mathrm{K} 1$ regardless of parametric uncertainty, the variation in rich solvent flowrate and composition is not large enough to have an appreciable effect on the stripper column simulation.

For the absorber column, the mass transfer model uncertainty appears to have the greatest effect on the $\mathrm{CO}_{2}$ capture percentage distribution when the column is in a mass transfer limited regime, 
at or near complete $\mathrm{CO}_{2}$ capture percentage. For Case $\mathrm{K} 1$, the distribution of $\mathrm{CO}_{2}$ capture percent resulting from the parametric uncertainty in the thermodynamic model is very narrow, but expands with the inclusion of mass transfer parametric uncertainty. A similar trend is shown for Case K18, in which absorber operation is slightly below complete $\mathrm{CO}_{2}$ capture. For Case K6, in which the column is operating well below complete $\mathrm{CO}_{2}$ capture, in a reaction controlled regime, the relative change in the distribution of column efficiency upon inclusion of mass transfer uncertainty is smaller. Comparing the distributions of $\mathrm{CO}_{2}$ capture percentage in Figure 6.14 with the data values in Table 6.3, it may be shown that the data values or at or near the respective regions of substantial probability density. The same observation is made for the predicted lean loading distributions and the data values given in Table 6.4.

The temperature profiles for absorber and stripper columns are also evaluated for the stochastic model, and the results are shown in Figures 6.15 and 6.16, respectively.

K1

A

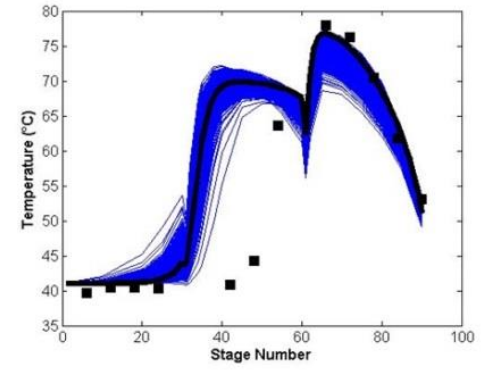

$\mathrm{B}$

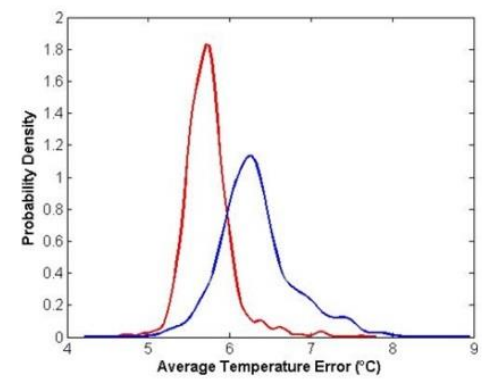

K6
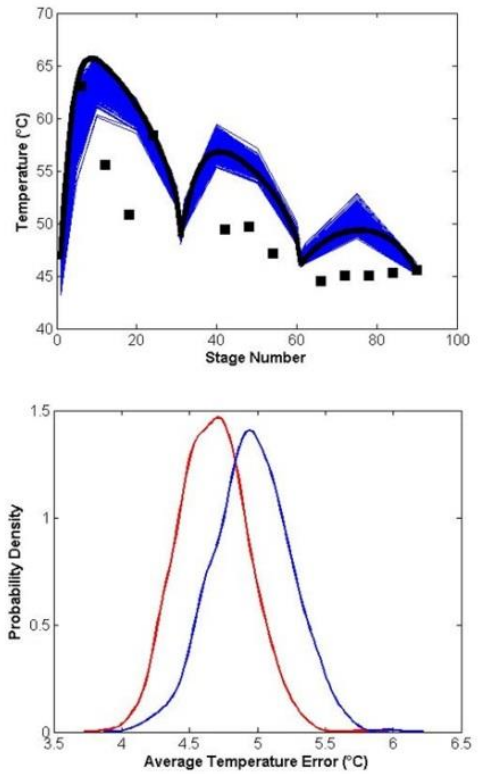

K18
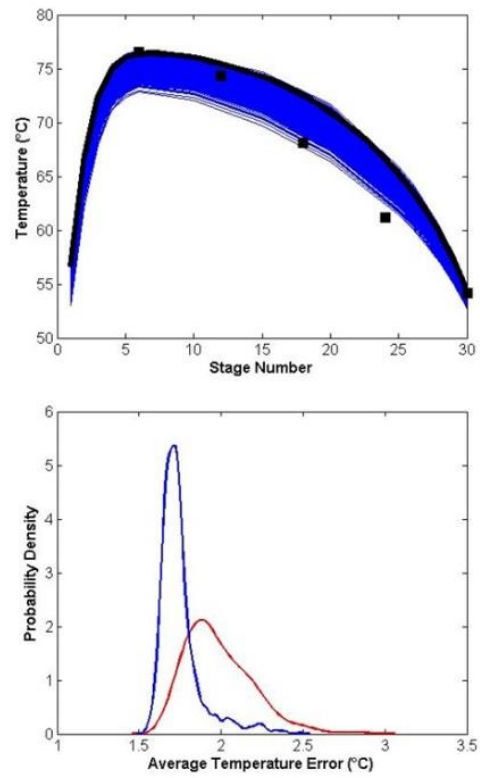

Figure 6-15. Calculated temperature profiles for absorber column stochastic model. (A) Deterministic model temperature profile represented by black line and temperature profiles calculated for realizations from parameter distribution given by blue lines (B) Estimated pdfs for average temperature profile error for stochastic simulation. Red lines represent case in which thermodynamic model parameter uncertainty is considered and blue lines represent case in which thermodynamic, mass transfer, and hydraulics model uncertainty is considered. 
$\mathrm{K} 1$

A

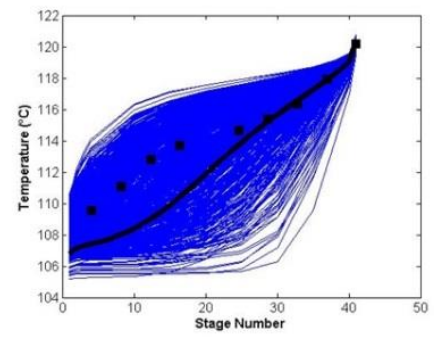

B

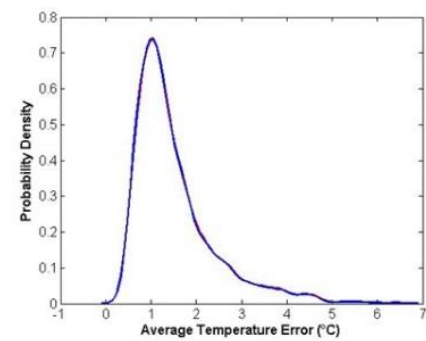

K6
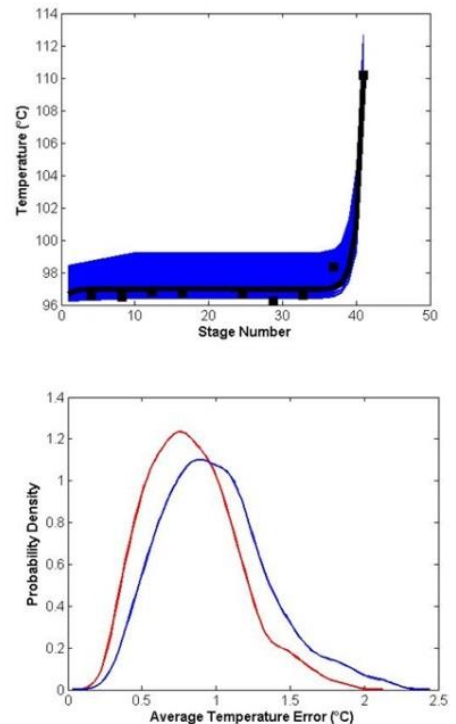

$\mathrm{K} 18$
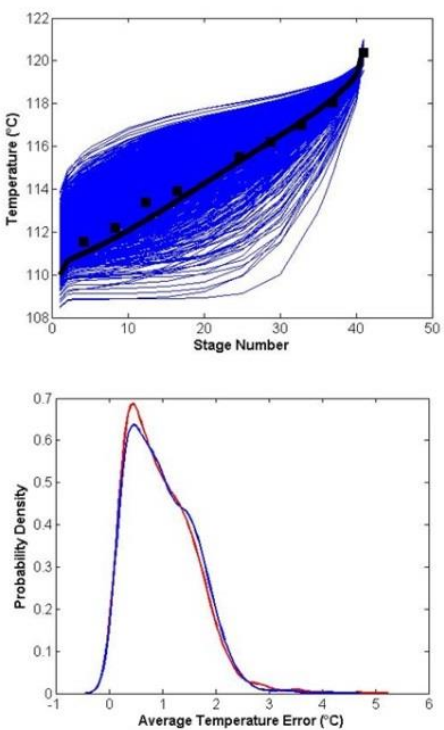

Figure 6-16. Calculated temperature profiles for stripper column stochastic model. (A) Deterministic model temperature profile represented by black line and temperature profiles calculated for realizations from parameter distribution given by blue lines (B) Estimated pdfs for average temperature profile error for stochastic simulation. Red lines represent case in which thermodynamic model parameter uncertainty is considered and blue lines represent case in which thermodynamic, mass transfer, and hydraulics model uncertainty is considered.

The temperature profile estimations for both columns tend to take on a rather wide range in many of the cases. For the stripper column, a wide range for the temperature within the column is shown, although the temperature value for the solvent outlet at the bottom of the column has a very narrow range in the stochastic simulation and matches the data value well for all of these cases. As for the process variables for which results are shown in Figure 6.12, the distributions of the average temperature profile error and the effect of the thermodynamic and mass transfer parametric uncertainty on them are highly case dependent.

The Sobol indices are also calculated for this analysis, and presented here in the normalized form:

$$
\hat{S}_{T i}=\frac{S_{T i}}{\sum_{i=1}^{k} S_{T i}}
$$

This normalization technique is used for the comparison because the denominator of Eq. 6.2 generally takes on a value greater than 1 that varies for different cases and for the two dependent 
variables. The parameter numbers are as assigned for the thermodynamic model parameters (1-9) in Table 6.6, mass transfer parameters (10-11) in Table 6.7, and the hydraulics model parameters (12-13) in Table 6.8. The Sobol indices are given for the $\mathrm{CO}_{2}$ capture percentage in Figure 6.17 and the outlet lean loading in Figure 6.18. 


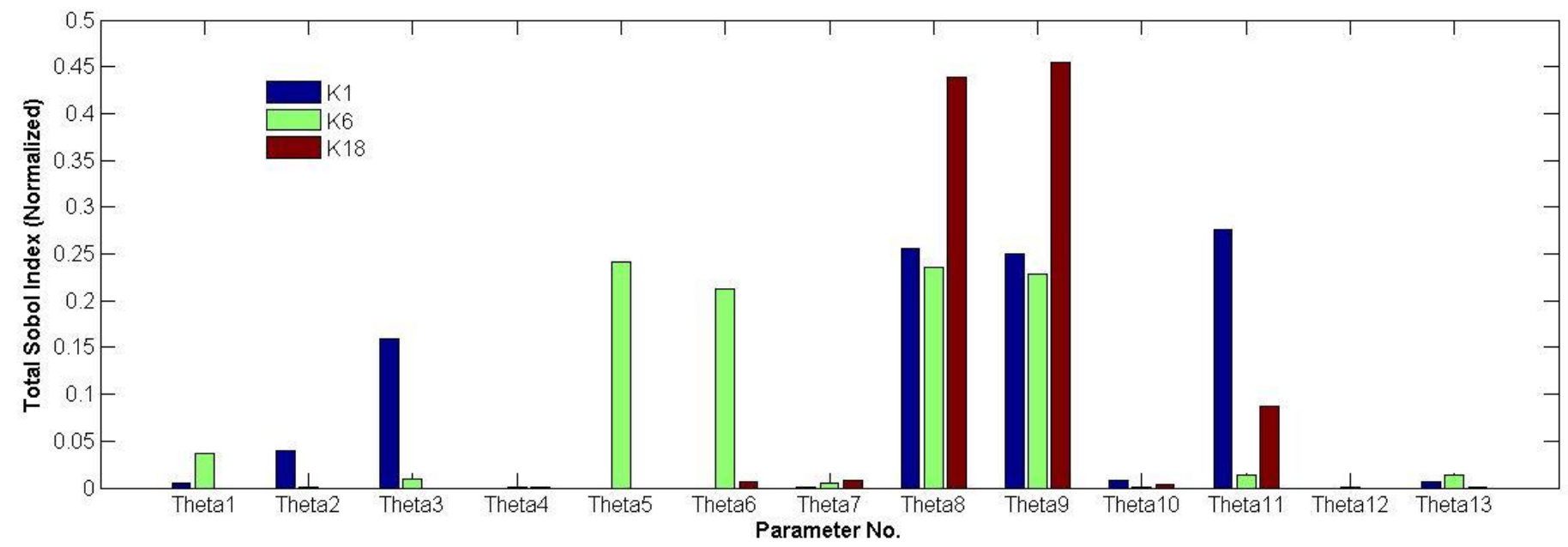

Figure 6-17. Values of Sobol indices for parameters with respect to $\mathrm{CO}_{2}$ capture percentage prediction for three cases

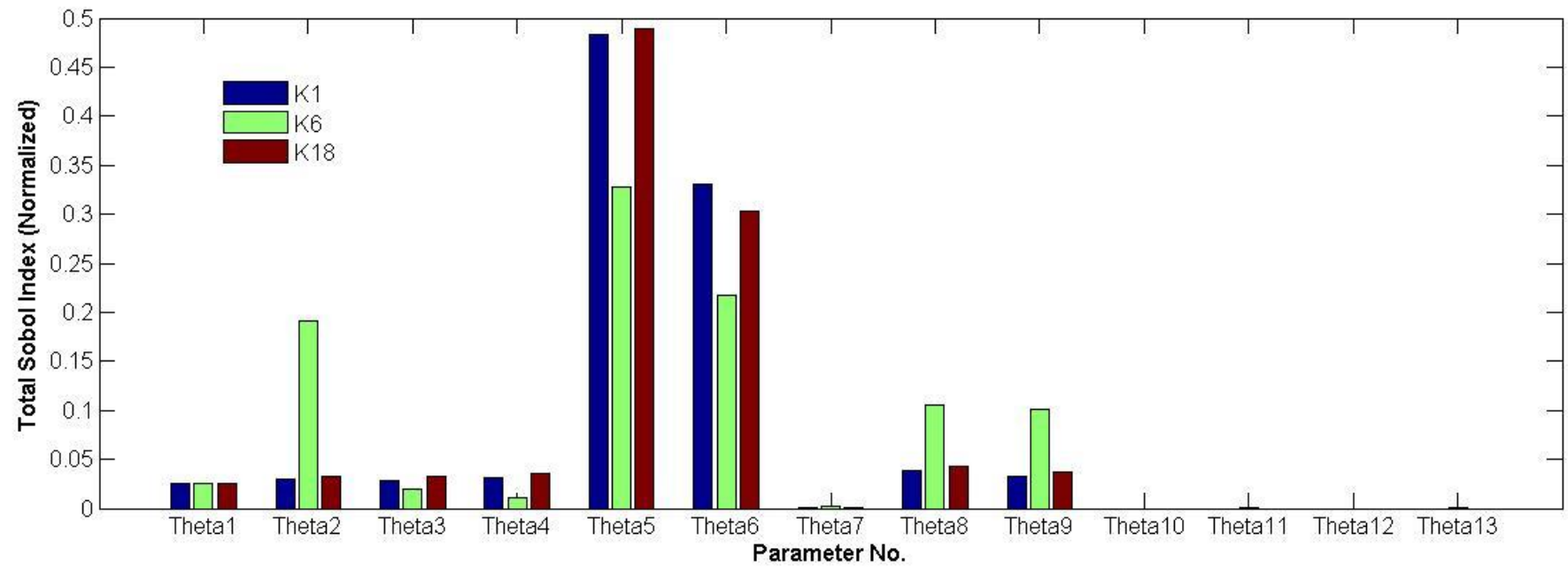

Figure 6-18. Values of Sobol indices for parameters with respect to lean loading prediction for three cases 
In the absorber simulation, the NRTL parameters (8-9) are shown to have the greatest effect on the $\mathrm{CO}_{2}$ capture prediction in general. The mass transfer parameters are shown to have some effect on the absorber simulation for Cases $\mathrm{K} 1$ and $\mathrm{K} 18$, and less effect on the simulation for Case K6, in which the absorber operates at a lower $\mathrm{CO}_{2}$ capture percentage. For the stripper, the mass transfer parameters are shown to have no appreciable effect on the performance of the column as evident from the negligibly small values of the Sobol indices. The Henry model parameters (5-6) are shown to have the largest effect on the calculation of the lean solvent loading. The hydraulics model parameters (12-13) are not shown to have a great effect on the uncertainty in either the absorber or stripper model. The Sobol indices are generally consistent with the results obtained from propagating only the thermodynamic model parameters through the process model and propagating all of the thermodynamic, mass transfer, and hydraulics model parameters and observing the distributions separately for the two cases.

\subsection{Measurement Uncertainty Analysis}

In this section of the work, a sensitivity study is performed to analyze the effect of the solvent concentration on the process model performance. For this analysis, the true values of the weight percentage of MEA and $\mathrm{CO}_{2}$ are taken to be related to the values given in the experimental data by the relationship:

$$
\frac{\gamma_{i}}{100}=\frac{\widehat{W}_{i}-W_{i}}{\widehat{W}_{i}}
$$

where $\widehat{W}_{i}$ is the data value of the weight percentage of species $i$ and $W_{i}$ is the hypothetical true value calculated with respect to a measurement error $\left(\gamma_{i}\right)$ percentage that is treated as a variable in this work. The measurement error values were estimated during the 2014 MEA solvent campaign at NCCC and the methodology is included in the drafted manuscript related to this campaign (Morgan et al., 2017b). The detailed results are not presented here as they were obtained as a part of a collaboration, and not collected as a part of the work performed for this dissertation. Average values were determined to be $\gamma_{M E A}^{*}=-0.22$ and $\gamma_{\mathrm{CO}_{2}}^{*}=-5.31$ and ranges were determined from a Bland-Altman plot analysis (Bland and Altman, 1986), and determined to be: 


$$
\begin{aligned}
& \gamma_{\mathrm{CO}_{2}} \in[-16.7,6.09] \\
& \gamma_{M E A} \in[-5.97,5.53]
\end{aligned}
$$

Since the measurement errors are given in terms of percentages and the lean solvent loading values from the data set from NCCC vary over a relatively wide range $\left(\alpha_{\text {lean }} \in[0.05,0.40]\right)$, the degree to which the measurement error effects the simulation results varies by case. For Case $\mathrm{K} 6$, notable for absorber operation with high inlet lean loading, the sensitivity of absorber $\mathrm{CO}_{2}$ capture percentage and stripper outlet lean loading to the assumed measurement error in $\mathrm{CO}_{2}$ and MEA composition is given in Figure 6.19. The reported values of percentage of lean loading error are calculated with respect not to the data value of lean loading but to the specified value of lean loading at the absorber inlet, which is a function of both values of assumed measurement error $\left(\gamma_{C_{2}}\right.$ and $\left.\gamma_{M E A}\right)$. Accordingly, the percentage of lean loading error essentially quantifies the discrepancy between lean loading specified at the absorber inlet and calculated at the stripper outlet. By convention, a positive value of lean loading error percentage indicates that the value calculated at the stripper outlet is greater than the value specified at the absorber inlet. 
A

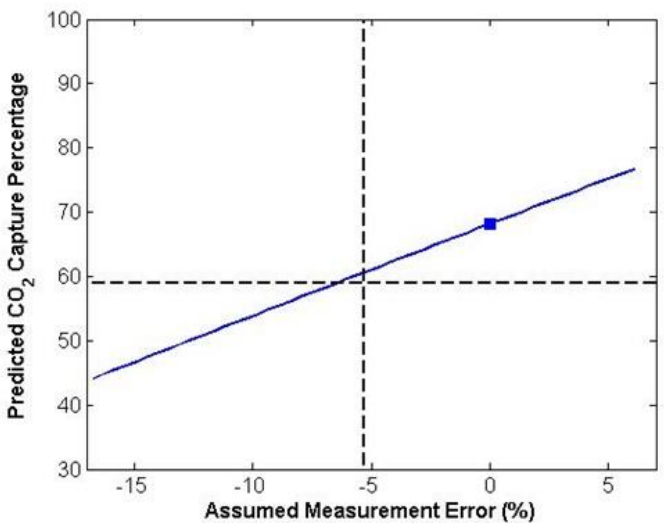

B

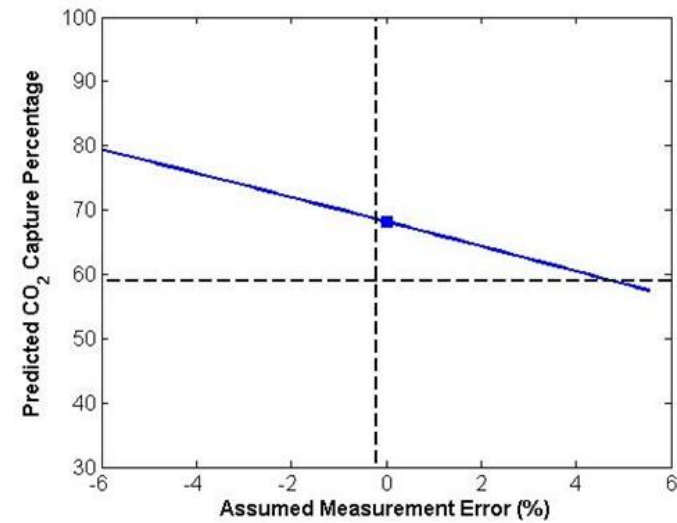

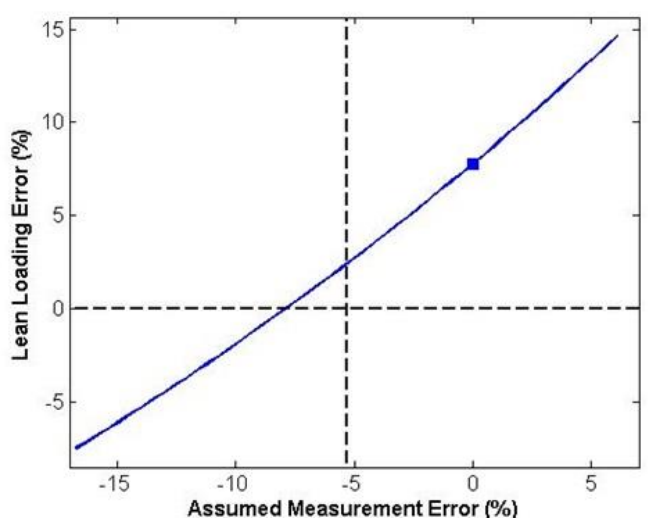

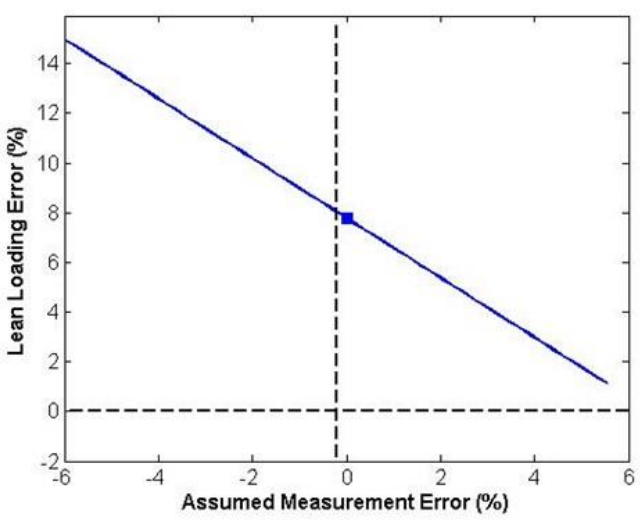

Figure 6-19. Results of sensitivity study for Case K6 in which effect of the measurement error assumption on calculated values of $\mathrm{CO}_{2}$ capture percentage in absorber and lean loading in stripper outlet is analyzed. Solid blue lines represent calculated values over measurement error ranges and blue squares used to highlight baseline model values. Dashed black lines used to denote data values of process variables (horizontal) and estimated average values of measurement error (vertical). Results are given for perturbations in (A) $\mathrm{CO}_{2}$ weight percentage and (B) MEA weight percentage.

The calculations of the process output variables are presented separately for perturbations in the weight fractions of $\mathrm{CO}_{2}$ and MEA. The $\mathrm{CO}_{2}$ capture percentage decreases as the assumed measurement error becomes more negative, indicating that the true $\mathrm{CO}_{2}$ composition of the lean solvent becomes large, resulting in reduced $\mathrm{CO}_{2}$ capture percentage. Although the $\mathrm{CO}_{2}$ capture percentage is overpredicted by about $10 \%$ for the baseline model $\left(\gamma_{\mathrm{CO}_{2}}=\gamma_{\mathrm{MEA}}=0\right)$, the discrepancy between model and data $\mathrm{CO}_{2}$ capture percentage becomes very small when the $\mathrm{CO}_{2}$ composition discrepancy is specified at the average value $\left(\gamma_{\mathrm{CO}_{2}}=\gamma_{\mathrm{CO}_{2}}^{*}=-5.31\right)$. This is represented in Figure 6.17 by the proximity of the $\mathrm{CO}_{2}$ capture curve with the intersection of the 
dashed lines. The error between the specified and calculated values of lean loading also becomes smaller as the same assumption is made regarding the $\mathrm{CO}_{2}$ concentration error, which is mostly attributed to an increase in the specified lean loading in the absorber inlet.

As for the $\mathrm{CO}_{2}$ composition measurement error, the $\mathrm{CO}_{2}$ capture percentage of the absorber also varies linearly with the assumed value of MEA composition measurement. The average value of the estimated measurement is approximately 0, indicating that no bias in the MEA composition is expected. As the assumed measurement error is increased, the specified amount of MEA in the lean solvent also decreases, resulting in simultaneous decrease in the prediction of $\mathrm{CO}_{2}$ capture percentage and error in the calculated value of lean loading.

The effect of the potential bias in $\mathrm{CO}_{2}$ composition in the solvent is analyzed for two additional cases, with the results given in Figure 6.20. 
K3
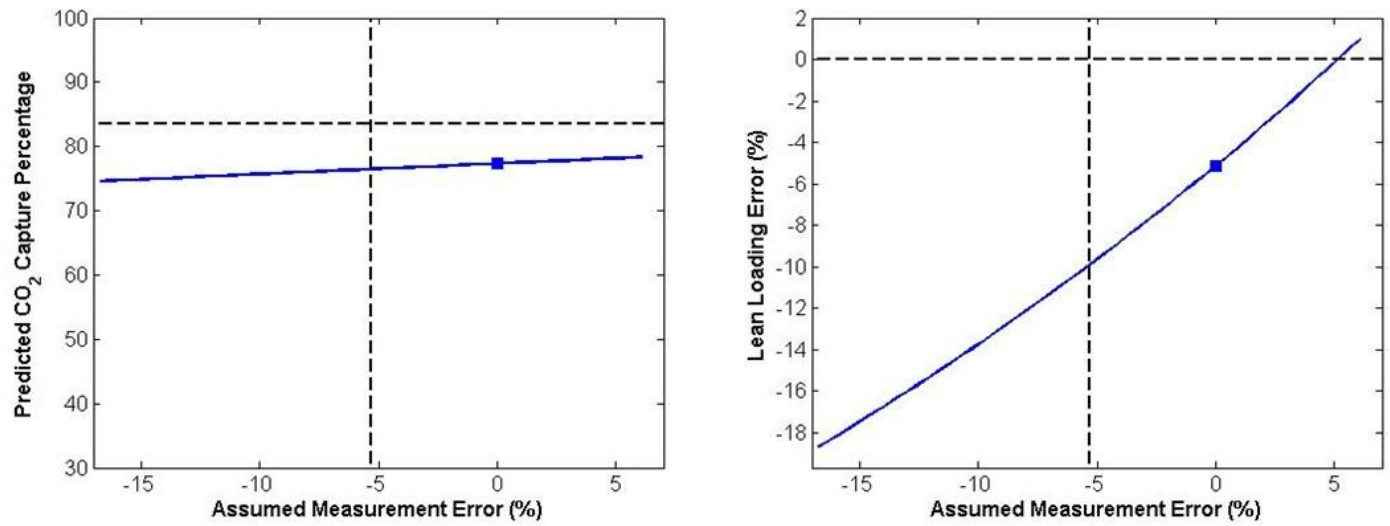

K7
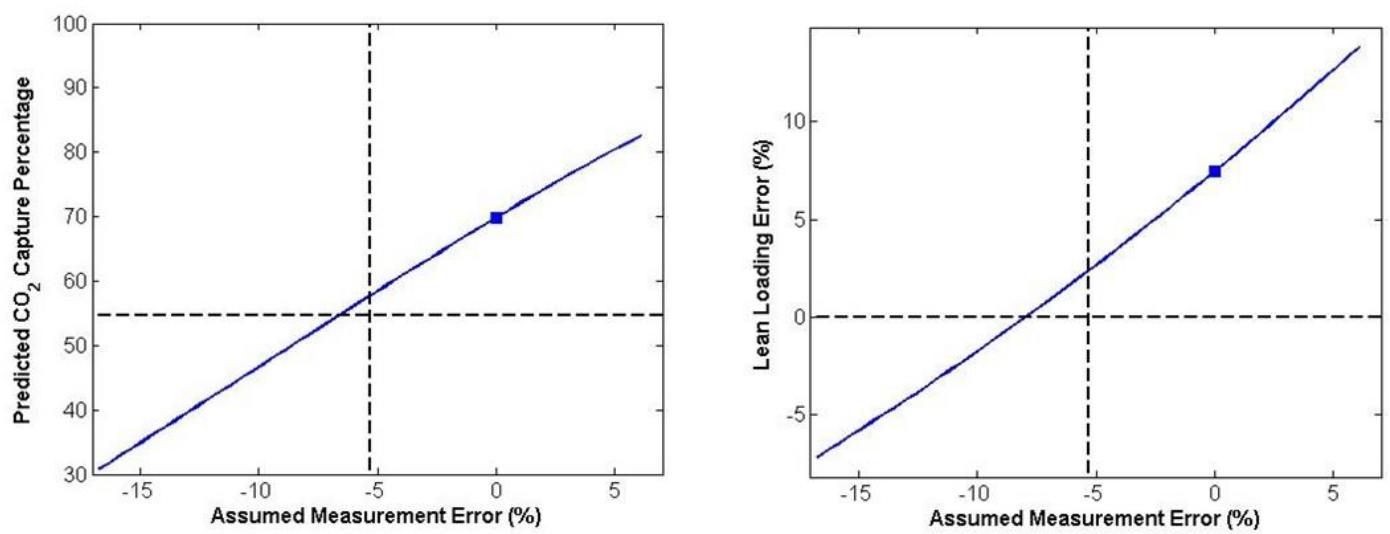

Figure 6-20. Results of sensitivity study in which predictions of $\mathrm{CO}_{2}$ capture and stripper outlet lean loading are analyzed as a function of assumed measurement error in $\mathrm{CO}_{2}$ weight percentage. Solid blue lines represent calculated values over measurement error ranges and blue squares used to highlight baseline model values. Dashed black lines used to denote data values of process variables (horizontal) and estimated average values of measurement error (vertical).

The results for Case $\mathrm{K} 7$ are similar to those for Case $\mathrm{K} 6$, as the $\mathrm{CO}_{2}$ capture percentage closely matches the data value and the lean loading error becomes very small if the measurement error in $\mathrm{CO}_{2}$ concentration is assumed to be at its average estimated value. For Case $\mathrm{K} 3$, the $\mathrm{CO}_{2}$ concentration error assumption has very little effect on the $\mathrm{CO}_{2}$ capture prediction for the absorber due to the very small value of lean loading, which changes minimally based on the percentage perturbation in the $\mathrm{CO}_{2}$ concentration. Although the percentage of lean loading error also increases along with the slight increase in the discrepancy of model and data $\mathrm{CO}_{2}$ capture percentage when the $\mathrm{CO}_{2}$ concentration bias is assumed, this is qualified by the small magnitude of the lean loading value. These examples give support to the plausibility of the bias in the $\mathrm{CO}_{2}$ 
concentration measurement, as the model and data values of $\mathrm{CO}_{2}$ capture percentage in the absorber and lean $\mathrm{CO}_{2}$ loading in the stripper outlet have decreased discrepancy when the $\mathrm{CO}_{2}$ concentration in the solvent is assumed to be underestimated by approximately $5.31 \%$.

All 23 cases are repeated assuming this discrepancy in the lean solvent $\mathrm{CO}_{2}$ concentration for determining the effect of the measurement uncertainty on the $\mathrm{CO}_{2}$ capture percentage of the absorber. A parity plot of the predicted $\mathrm{CO}_{2}$ capture percentage with and without the assumption of the $\mathrm{CO}_{2}$ concentration error is given in Figure 6.21 for all 23 steady state data points. The comparison is also made for the number of transfer units (NTU), which is calculated from a conversion of the $\mathrm{CO}_{2}$ capture percentage $\left(\mathrm{CO}_{2, \mathrm{cap}}\right)$ :

$$
N T U=-\ln \left(1-\frac{C O_{2, c a p}}{100}\right)
$$

A

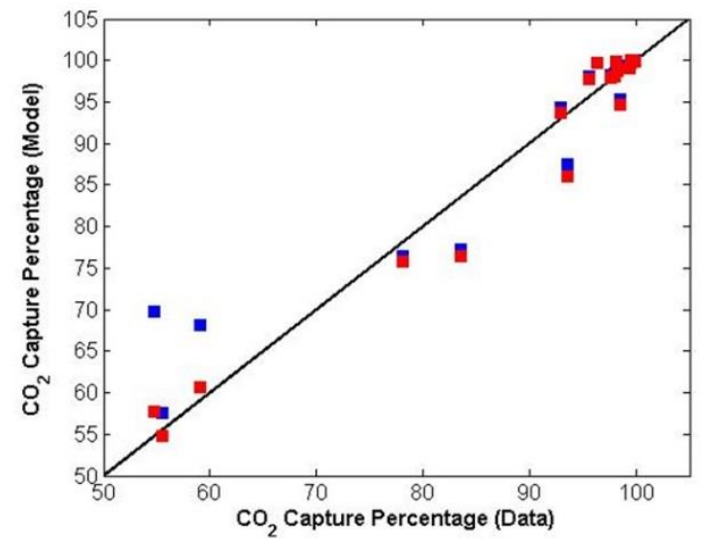

B

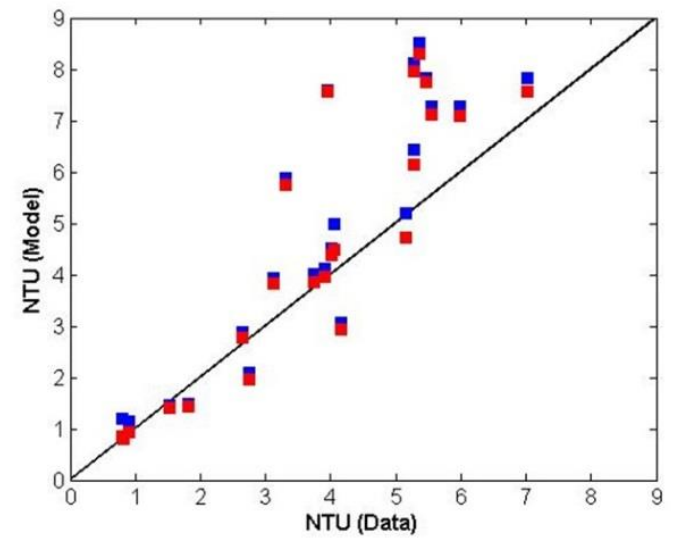

- Without $\mathrm{CO}_{2}$ Concentration Discrepancy

- With $\mathrm{CO}_{2}$ Concentration Discrepancy

Figure 6-21. Parity plots for (A) $\mathrm{CO}_{2}$ capture percentage and (B) NTU for absorber column simulation with and without assumption of $\mathrm{CO}_{2}$ concentration discrepancy.

As shown in Figure 6.21, the major effect of assuming the $\mathrm{CO}_{2}$ concentration discrepancy is a reduction in the prediction error for the cases with high lean $\mathrm{CO}_{2}$ loading and low $\mathrm{CO}_{2}$ capture, namely $\mathrm{K} 6$ and $\mathrm{K} 7$ as discussed earlier. For the region of higher $\mathrm{CO}_{2}$ capture percentage, the model discrepancy increases from the NTU perspective since this transformation is intended to penalize the error in this region. However, this does not consider the effect of measurement uncertainty in the $\mathrm{CO}_{2}$ capture percentage, which could lead to high reported error in NTU given the sensitivity of this measure at high $\mathrm{CO}_{2}$ capture. 


\subsection{TCM Test Campaign}

Although the validation work for the MEA model has been performed most rigorously with the NCCC data, the submodels of the solvent model developed for this work have also been incorporated into a process model developed for the pilot plant at the Technology Centre Mongstad (TCM) in Norway. Although the author of this dissertation was not directly involved in the TCM campaign or the validation work, these are included here as they provide further evidence of the validity of our MEA model at multiple scales. The TCM pilot plant is of considerably larger scale than the NCCC plant, and some comparison is given in Table. Moreover, this pilot plant provides access to two different types of flue gas, which are from a Residue Catalytic Cracker (RCC) and a natural gas combined heat and power (CHP) plant.

Table 6-9. Comparison of NCCC and TCM pilot plants

\begin{tabular}{ccccccc}
\hline & $\begin{array}{c}\mathrm{CO}_{2} \\
\text { Capacity } \\
\text { (tpd) }\end{array}$ & $\begin{array}{c}\text { Source of } \\
\text { Flue Gas }\end{array}$ & & Absorber & & Stripper \\
& & & Diameter $(\mathrm{m})$ & $\begin{array}{c}\text { Height } \\
(\mathrm{m})\end{array}$ & $\begin{array}{c}\text { Diameter } \\
(\mathrm{m})\end{array}$ & Height $(\mathrm{m})$ \\
\cline { 3 - 7 } $\mathrm{NCCC}$ & 10 & Coal & 0.64 & 18.5 & 0.59 & 12.1 \\
TCM & $80-240$ & CHP, RCC & 3 & 24 & CHP: 1.25 & 8 \\
& & & & & RCC: 2.17 & \\
\hline
\end{tabular}

The absorber column at TCM is rectangular, and the diameter is presented as an equivalent diameter given the cross-sectional area of the column. Validation data for the MEA system were obtained during a 2015 campaign at TCM, and these data have previously been reported in Gjernes et al. (2016). In this work, the absorber and stripper columns are simulated separately, similarly to the methodology used for the validation with data from NCCC. The CCSI model prediction of $\mathrm{CO}_{2}$ capture percentage is compared to the experimental data for two cases in Table 6.10 . 
Table 6-10. Process variable values for two TCM test cases

\begin{tabular}{ccc}
\hline & Case 2B1 & Case 2B6 \\
\hline Lean Solvent Flowrate $(\mathrm{kg} / \mathrm{hr})$ & 71060 & 57130 \\
L:G Mass Ratio & 1.24 & 0.75 \\
Lean Loading (mol CO$/ \mathrm{MEA})$ & 0.309 & 0.192 \\
Rich Loading (mol CO$/ \mathrm{MEA})$ & 0.487 & 0.495 \\
Reboiler Duty $(\mathrm{kW})$ & 2751 & 2721 \\
Flue Gas $\mathrm{CO}_{2}$ Weight Percent & 5.5 & 5.5 \\
$\mathrm{CO}_{2}$ Capture Percentage (Data) & 84.29 & 90.59 \\
$\mathrm{CO}_{2}$ Capture Percentage (Model) & 84.86 & 87.78 \\
\hline
\end{tabular}

The comparisons of the absorber and regenerator temperature profiles are given for the two cases in Figures 6.22-23. Note that the columns include four temperature sensors across the cross section, and these are labeled as A-D in the figure. A bed height of 0 refers to the top of the column.

Case 2B1

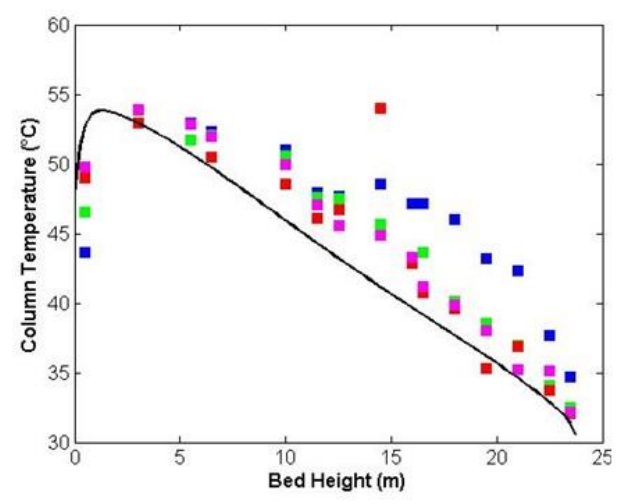

Case 2B6

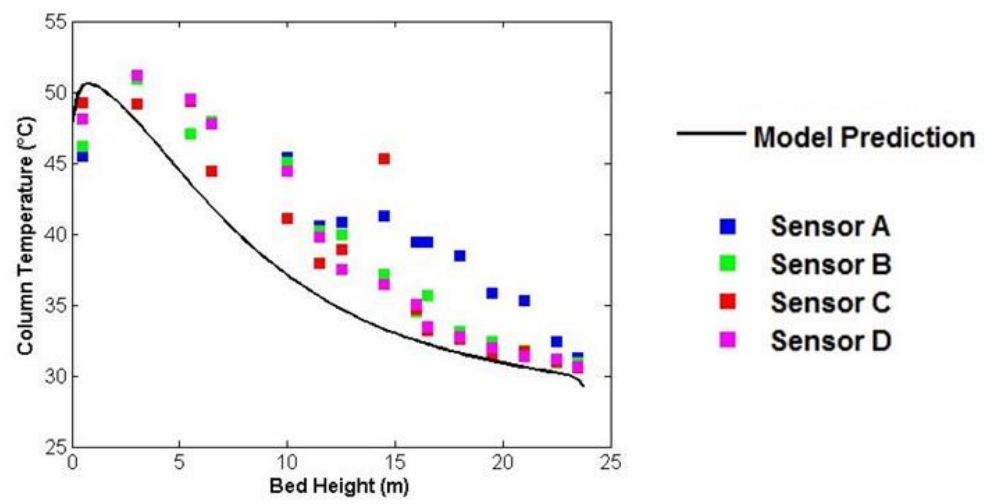

Figure 6-22. Comparison of TCM absorber temperature profiles for two cases. 

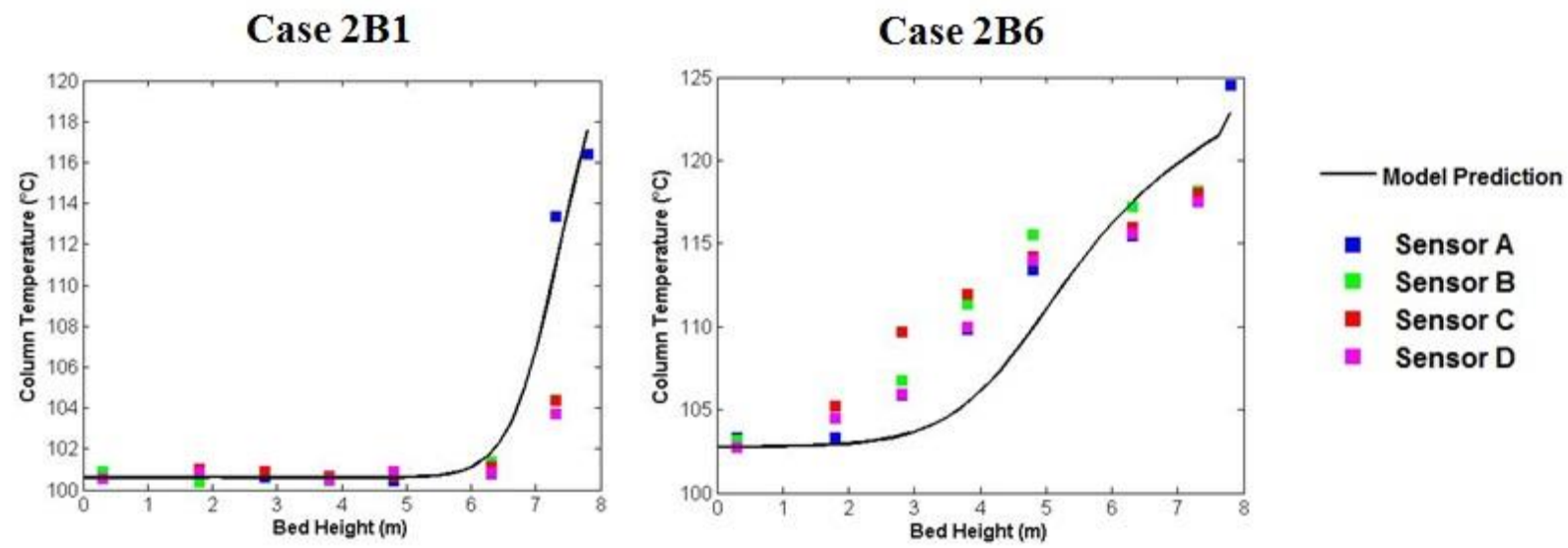

Figure 6-23. Comparison of TCM stripper temperature profiles for two cases

As for the NCCC case, some discrepancy between the model and data temperature profiles exists for the absorber and stripper columns, although the model captures the general shape of the temperature profile. Moreover, the absorber temperature appears to vary widely over the absorber cross section throughout the entire length of the column, although some of this variation may be attributed to sensor noise. It should be noted that as the model is 1-d, cross sectional variation of transport variables is ignored. Furthermore, the thermocouples are not uniformly located along the cross sectional area so it is hard to comment on the accuracy of the model. However for these as well as several other temperature profiles that were simulated, the model could capture the general trend similar to that shown above. Experimental data also exist for the $\mathrm{CO}_{2}$ loading profiles in the absorber column, and the model and data comparison for this variable is given in Figure 6.24. 
Case 2B1

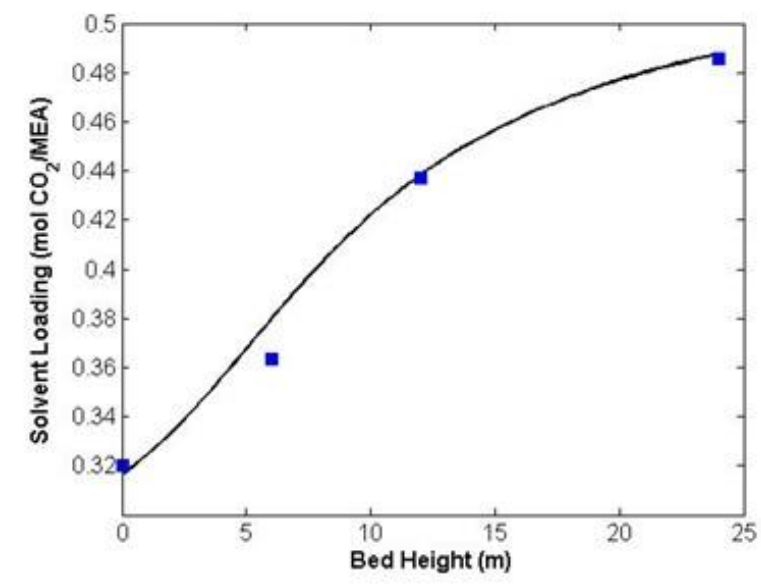

Case 2B6

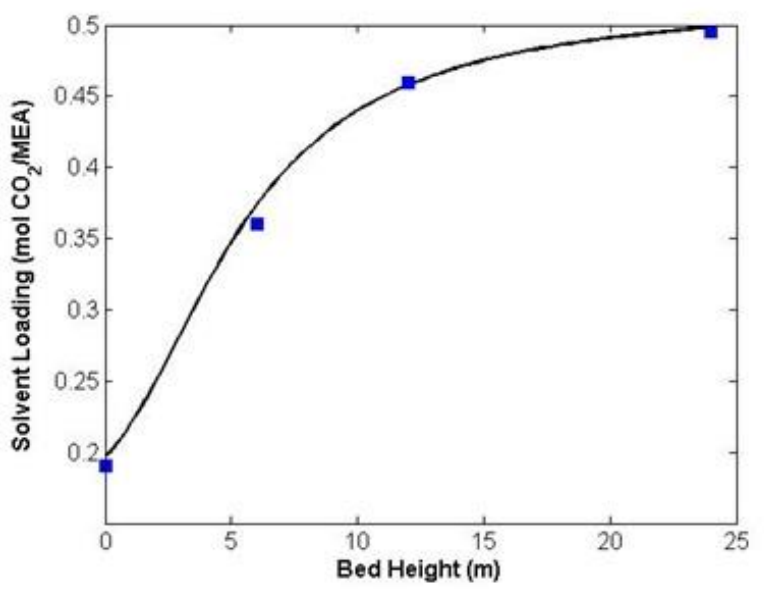

Figure 6-24. Comparison of TCM absorber lean loading profiles for two cases

The model prediciton of the lean loading in the absorber column for the two cases is shown to match the experimental data very accurately, although few data sensors are placed in the column. The results given here provide further insight into the ability of the solvent model to predict large-scale plant performance.

\subsection{Conclusions}

A complete process model of the $\mathrm{CO}_{2}$ capture process with aqueous MEA solution has been developed by combining physical property, reaction kinetics, mass transfer, and hydraulic submodels. The overall model has been validated with steady-state data obtained from a MEA solvent test campaign at NCCC, which was planned to cover a large range of operating conditions for the absorber and stripper columns. The model has been shown to match accurately the large scale pilot plant data, including absorber $\mathrm{CO}_{2}$ capture percentage and $\mathrm{CO}_{2}$ loading in the regenerator outlet as well as the temperature profiles for both columns.

Along with measurement of key process variables, a methodology was used for estimating the uncertainty in solvent composition by comparing the NCCC process measurements of MEA and $\mathrm{CO}_{2}$ concentration to laboratory methods of a known uncertainty. The consideration of this measurement sensitivity provides some insight into the discrepancy between the pilot plant data 
and the corresponding model predictions. It is shown that the match between the data and model predictions may be improved for some cases, particularly cases with high inlet $\mathrm{CO}_{2}$ concentration, if measurement error is assumed to be the average value estimated by this methodology. The comparison of NTU for the absorber column has shown that model error exists in the operating region of high $\mathrm{CO}_{2}$ capture percentage, although a more precise measurement of $\mathrm{CO}_{2}$ capture percentage would be required to make this comparison meaningful.

Parameter distributions for thermodynamic and mass transfer models are propagated through the process model for several of the NCCC cases. This allows for the uncertainty in key process variables to be estimated in light of the uncertainty in submodel parametric uncertainty. The relative importance of the uncertainty of each parameter on the process outputs is determined by calculating Sobol indices for the parameters. The indices for the parameters are shown to be highly dependent on the specific output variable of interest as well as the operating regime of the process.

Finally, the predictability of the model is analyzed with respect to varying scale by considering its performance in comparison to data from the larger TCM pilot plant. The absorber and stripper columns for this process are modeled using the submodels developed for the CCSI solvent model. A comparison of the performance of the process model for the columns has shown that the model is predictive of the $\mathrm{CO}_{2}$ capture percentage in the absorber and the amount of $\mathrm{CO}_{2}$ regeneration in the stripper, as well as the column profiles. In the following chapter, the ongoing work related to a second NCCC campaign and an attempt to improve the test run planning in order to obtain a better set of validation data is described. 


\section{Chapter 7 : NCCC MEA Test Campaign (Summer 2017) Planning}

\subsection{Motivation}

In ongoing work, a second MEA test campaign at NCCC has been performed in the summer of 2017. One of the major goals of this project is to use the MEA process model to design a test campaign to maximize the value of the data obtained. The 2014 MEA campaign at NCCC was designed using a space-filling approach without considering the output space, and the information obtained from the resulting data was not leveraged for updating the test plan. As a result, a large amount of data were collected for absorber operation at very high $\mathrm{CO}_{2}$ capture percentage (>99\%), and this clustering could have been avoided if a preliminary model had been considered for planning the test run.

For this work, a sequential Bayesian design of experiments (DOE) is proposed for maximizing the information content from the test runs, making use of the MEA solvent model developed in this work with due consideration of the parametric and measurement uncertainty. Bayesian DOE may be defined as the use of prior information in the planning of an experiment (Weaver et al., 2016). A review of previous work on Bayesian DOE may be found in Chaloner and Verdinelli (1995). To the best of our knowledge, such an approach has not been applied to a large-scale pilot plant test campaign for $\mathrm{CO}_{2}$ capture. A schematic of the proposed DOE for this test campaign is shown in Figure 7.1. 


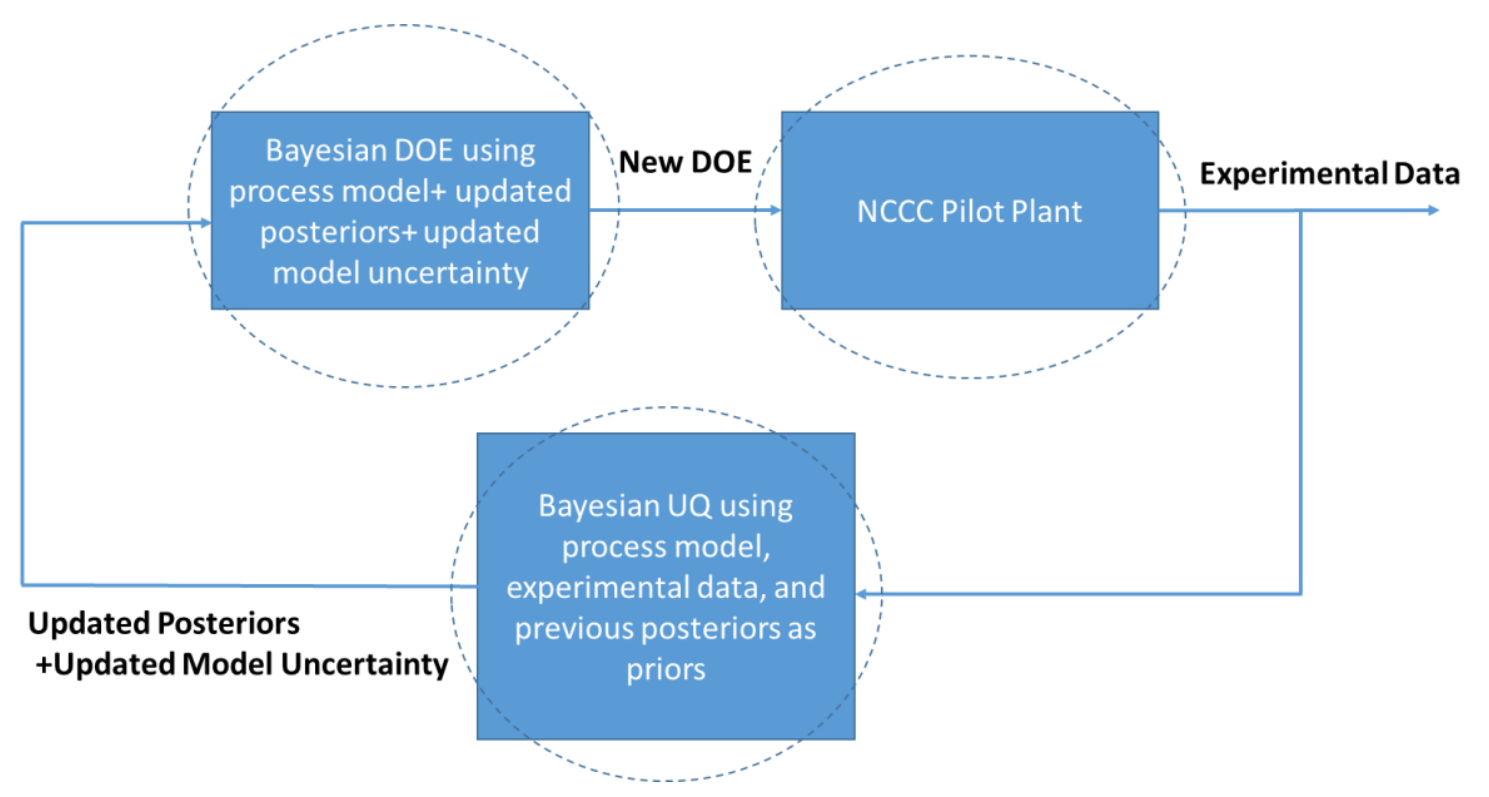

Figure 7-1. Schematic of proposed Bayesian DOE for MEA campaign at NCCC

In this approach, an estimate of the uncertainty in the process model prediction of an output is generated prior to the planning stage of the experiment. For this work, the uncertainty in the $\mathrm{CO}_{2}$ capture percentage of the absorber, which is considered as the major output variable of interest for this project, is estimated by propagating the parametric uncertainty from the submodels from the process model. The DOE methodology is used to develop a test plan for experiments to be run at the NCCC pilot plant. The resulting experimental data are incorporated into a Bayesian inference methodology, similar to that described in Chapter 2 for the process submodels, so that the distributions of some of the model parameters may be updated. As the model parameter distributions, and thus the estimation of the uncertainty in the model predictions, are updated in light of the experimental data, a new test plan may be developed.

\subsection{Deterministic Model Performance}

\subsubsection{Absorber Column (3 Beds with Intercooling)}

In preparation for planning the test campaign, a more rigorous analysis of the absorber operation over a wide range of operating conditions is considered. There are five possible test configurations for the absorber column at NCCC, given that the column consists of three beds, 
each separated by intercoolers. The configurations are three beds with and without intercooling, two beds with and without intercooling, and one bed without intercooling. As with the 2014 campaign, this work focuses on the three beds with intercooling process configuration due to the limitation in the amount of data that can be collected. It is also planned to collect some data for the remaining four process configurations so that the model's applicability in various process configurations can be tested further. In this project, test runs are planned with respect to four major input variables: the lean solvent flowrate $(L)$, flue gas flowrate $(G)$, solvent lean loading $\left(\alpha_{\text {lean }}\right)$, and $\mathrm{CO}_{2}$ weight fraction in the flue gas $\left(w_{\mathrm{CO}_{2}}\right)$. These variables are given the following constraints for this test campaign:

$$
\begin{gathered}
L \in[3000-13000] \mathrm{kg} / \mathrm{hr} \\
G \in[1000-3000] \mathrm{kg} / \mathrm{hr} \\
\alpha_{\text {lean }} \in[0.1-0.3] \mathrm{mol} \mathrm{CO} / \mathrm{MEA} \\
w_{\mathrm{CO}_{2}} \in[0.125-0.175]
\end{gathered}
$$

These ranges are based on the overall ranges for which data were given in the 2014 test campaign. A few of the previous data contain lean loading either above or below the range given here, although $0.3 \mathrm{~mol} \mathrm{CO} / \mathrm{MEA}$ has been determined to be a reasonable cut-off due to the high inefficiency of operating the absorber column at a higher loading. On the other hand, operation for lean loading below $0.1 \mathrm{~mol} \mathrm{CO}_{2} / \mathrm{MEA}$ would result in a relatively high reboiler duty requirement in the stripper column, and thus a high cost of operation.

For the three beds with intercooling case, the width of the $95 \%$ confidence intervals of $\mathrm{CO}_{2}$ capture is estimated over the input variable ranges of interest by propagating the parametric uncertainty, for the 13 submodel parameters indicated in Chapter 6.3.2 through the absorber model. A sensitivity study is performed to quantify the effect of the process variables on the absorber efficiency. The $\mathrm{CO}_{2}$ capture percentage is calculated as a function of lean solvent flowrate, lean loading, and $\mathrm{CO}_{2}$ weight fraction for a fixed baseline value $(2250 \mathrm{~kg} / \mathrm{hr})$ of flue gas flowrate in Figure 7.2. The MEA weight fraction in the lean solvent, on a $\mathrm{CO}_{2}$-free basis, is specified as $30 \%$, although may be considered as a disturbance variable in the actual process. 

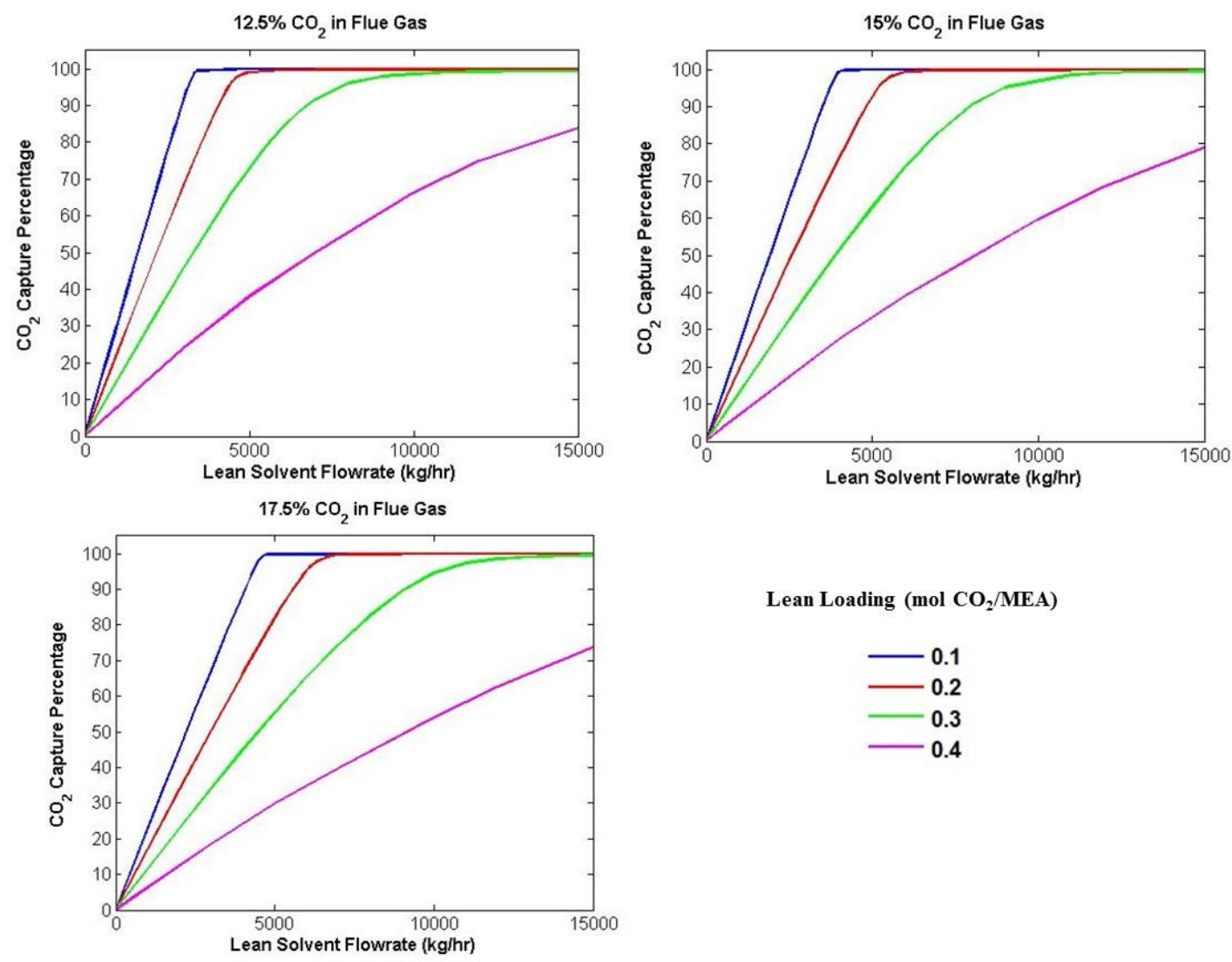

Lean Loading (mol $\left.\mathrm{CO}_{2} / \mathrm{MEA}\right)$

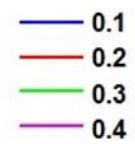

Figure 7-2. Effect of lean solvent flowrate on $\mathrm{CO}_{2}$ capture percentage in absorber with 3 beds and intercooling, with variable $\mathrm{CO}_{2}$ loading in solvent and $\mathrm{CO}_{2}$ weight fraction in flue gas.

For a given $\mathrm{CO}_{2}$ loading in the solvent and $\mathrm{CO}_{2}$ weight fraction in the flue gas, it is shown that the absorber efficiency increases approximately linearly with increasing solvent flowrate. At a sufficiently high flowrate, the capture percentage asymptotically approaches complete $\mathrm{CO}_{2}$ capture with increasing flowrate. Since the column efficiency decreases with increasing $\mathrm{CO}_{2}$ loading in the inlet solvent stream, the required liquid flowrate to reach complete $\mathrm{CO}_{2}$ capture also increases monotonically with inlet solvent loading. Although $0.3 \mathrm{~mol} \mathrm{CO}_{2} / \mathrm{MEA}$ has been chosen as a cutoff point for this work, $0.4 \mathrm{~mol} \mathrm{CO}_{2} / \mathrm{MEA}$ is included in the figure to indicate the dramatic decrease in column efficiency that occurs at very high values of lean loading in the column. With all other variables held constant, the $\mathrm{CO}_{2}$ capture percentage also decreases as the amount of $\mathrm{CO}_{2}$ in the flue gas increases, although the sensitivity to this variable over the range of interest is relatively small in comparison to the other input variables. 
Although the flue gas flowrate is at a fixed value, which has been chosen due to it being a common baseline value in the 2014 NCCC campaign, although the model is also evaluated along a range for this variable, also determined from the 2014 campaign. The sensitivity of the absorber efficiency to the flowrates of the lean solvent and flue gas are shown for three distinct values of lean loading in Figure 7.3.
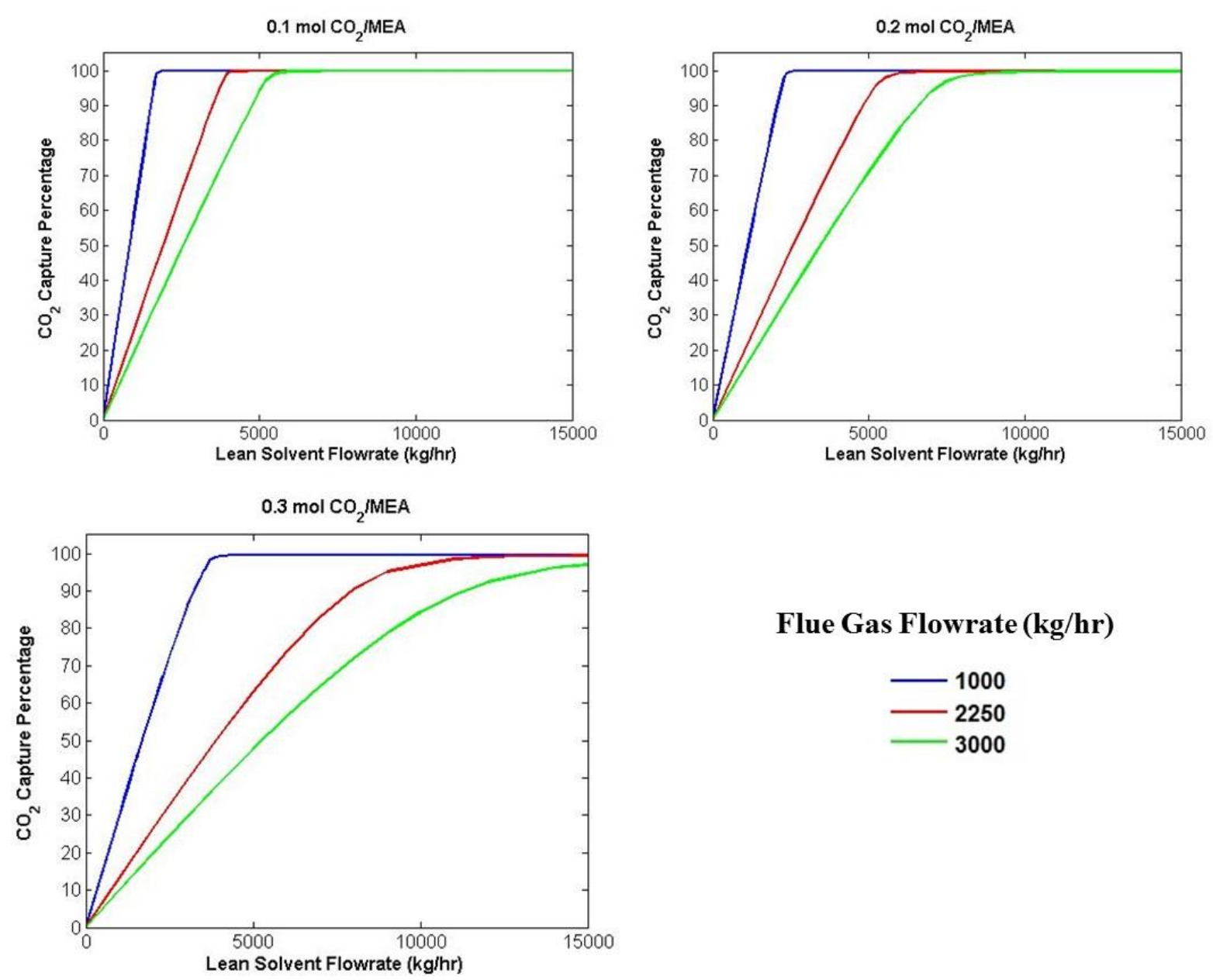

Flue Gas Flowrate (kg/hr)

Figure 7-3. Effect of lean solvent flowrate, flue gas flowrate, and lean solvent loading on $\mathrm{CO}_{2}$ capture percentage of absorber. $\mathrm{CO}_{2}$ weight percent in flue gas is fixed at $15 \%$.

As expected, the $\mathrm{CO}_{2}$ capture percentage decreases with increasing flue gas flowrate, as the amount of $\mathrm{CO}_{2}$ to be captured is higher. The same comparison is shown in Figure 7.4, although the ratio between liquid and gas flowrates into the absorber is compared in lieu of the total liquid flowrate. 

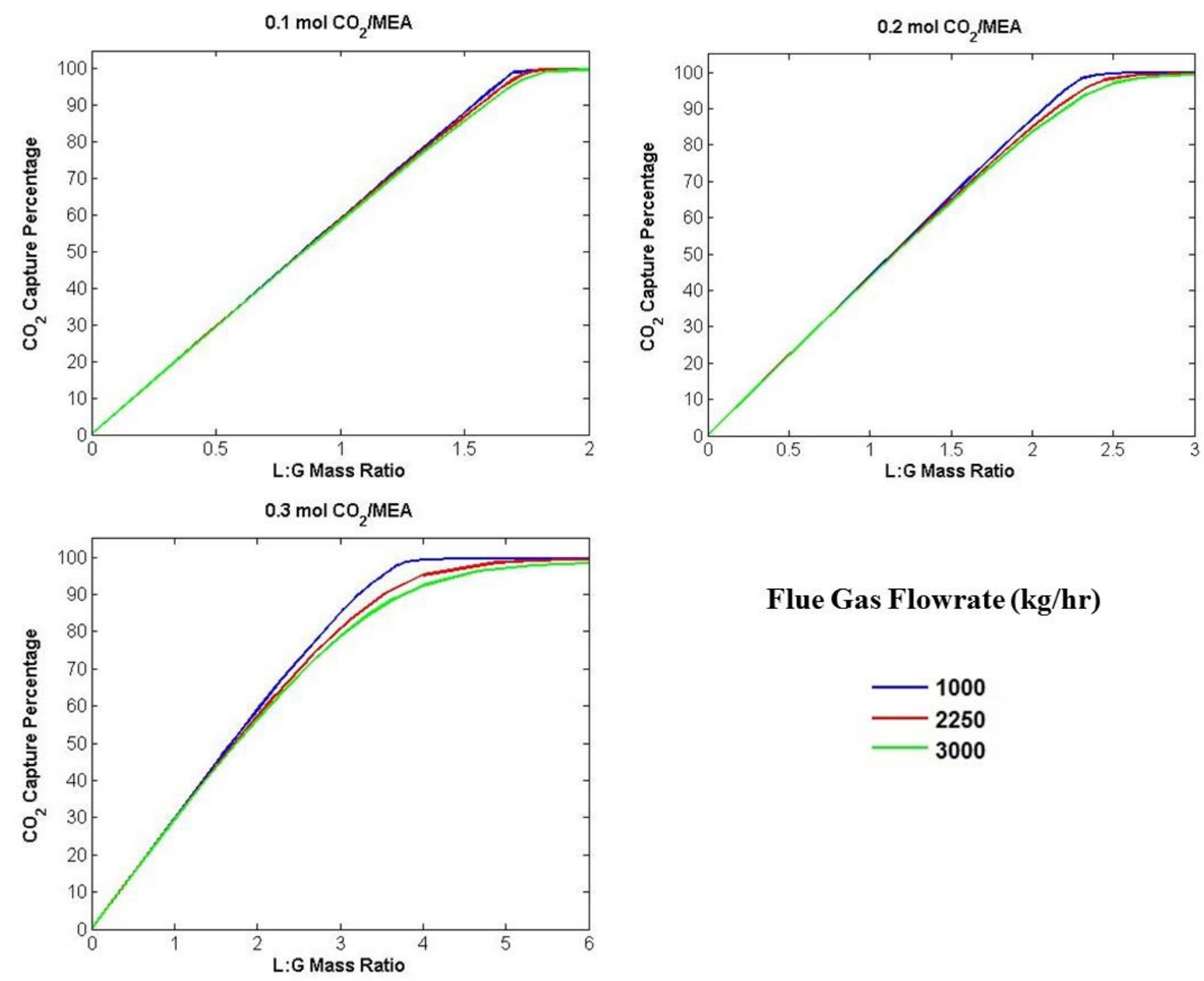

Flue Gas Flowrate (kg/hr)

Figure 7-4. Effect of liquid to gas (L:G) ratio, flue gas flowrate, and lean solvent loading on $\mathrm{CO}_{2}$ capture percentage of absorber

As shown in Figure 7.4, the absorber efficiency may be approximated as a function of the L:G ratio over the operating range of interest, regardless of the magnitude of the actual flowrates. This approximation is particularly valid at low values of lean loading, and the discrepancy at higher values of lean loading is largest around the range of $\mathrm{L}: \mathrm{G}$ in which the column transitions to a mass-transfer limited regime and complete $\mathrm{CO}_{2}$ capture.

The information provided in the sensitivity studies given in this work is used to determine ranges of desired operation for the lean solvent flowrate as a function of the flue gas flowrate and $\mathrm{CO}_{2}$ composition, and the lean solvent loading. Since many of the test runs in the 2014 campaign gave absorber operating in the mass transfer limited regime, with capture percentage higher than $99 \%$, it is desired to select test runs for $\mathrm{CO}_{2}$ capture percentage between $50-95 \%$ for this 
campaign. In order to do so, the input space is constrained by quantifying the range of lean solvent, for given values of flue gas flowrate, $\mathrm{CO}_{2}$ weight fraction in the flue gas, and lean solvent loading, for which this constraint on absorber efficiency is met. These ranges are evaluated at discrete points of the input variables, given by:

$$
\begin{gathered}
G=[1000,2250,3000] \mathrm{kg} / \mathrm{hr} \\
\alpha_{\text {lean }}=[0.1,0.2,0.25,0.3] \mathrm{mol} \mathrm{CO} / \mathrm{MEA} \\
w_{\mathrm{CO}_{2}}=[0.125,0.15,0.175]
\end{gathered}
$$

A trilinear interpolation procedure is used to estimate the upper and lower bounds of lean solvent flowrate over the entire ranges of interest of these variables. The constraints on lean solvent flowrate are given in Figures 7.5-7. 

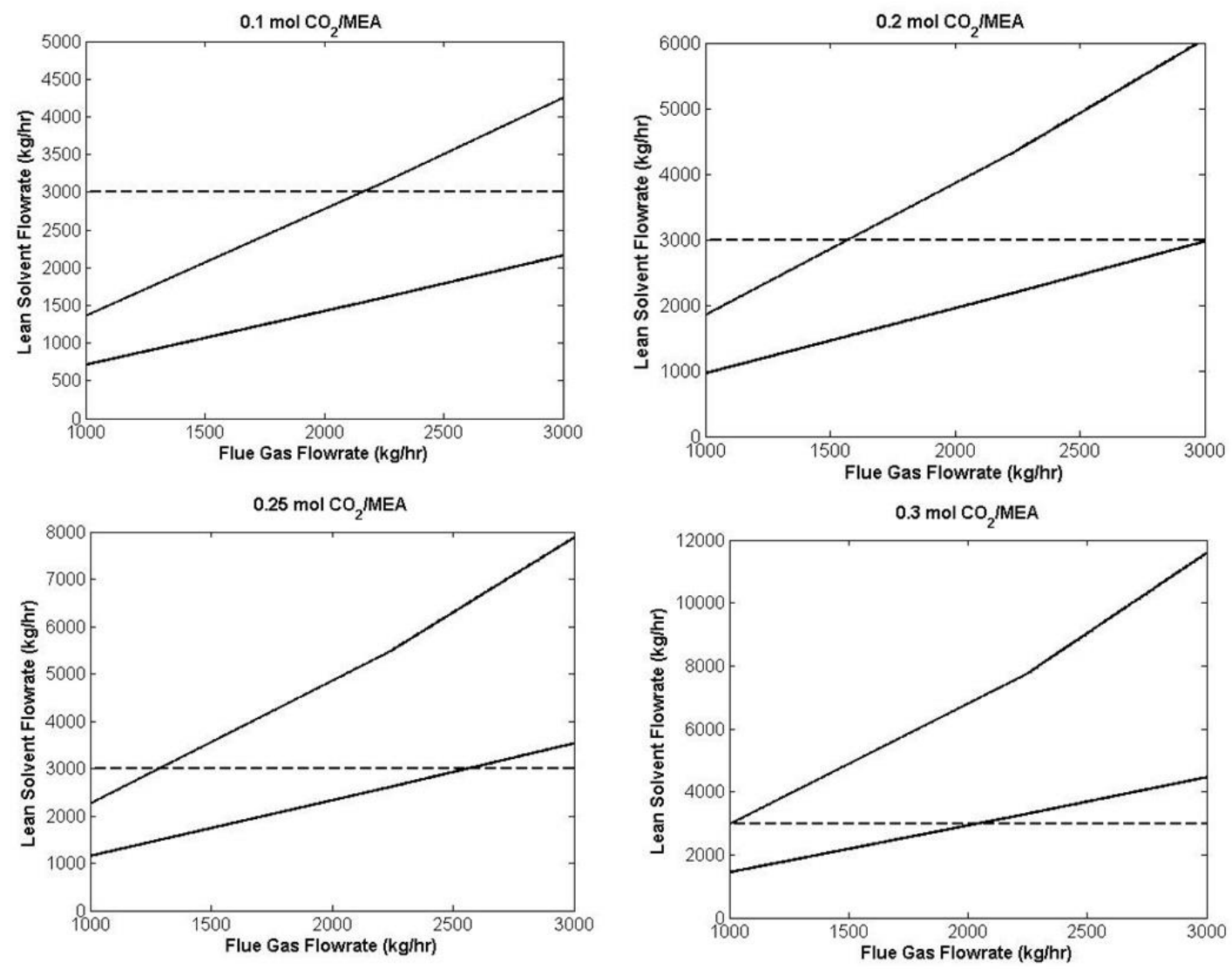

Figure 7-5. Estimated ranges of liquid flowrate, for a given value of lean loading and 12.5 wt\% $\mathrm{CO}_{2}$ in flue gas, for which $\mathrm{CO}_{2}$ capture percentage is constrained between $50-95 \%$. Solid lines represent the ranges of liquid flowrate for which requirement is met, and dashed line is included to illustrate additional constraint that liquid flowrate remains above $3000 \mathrm{~kg} / \mathrm{hr}$. 

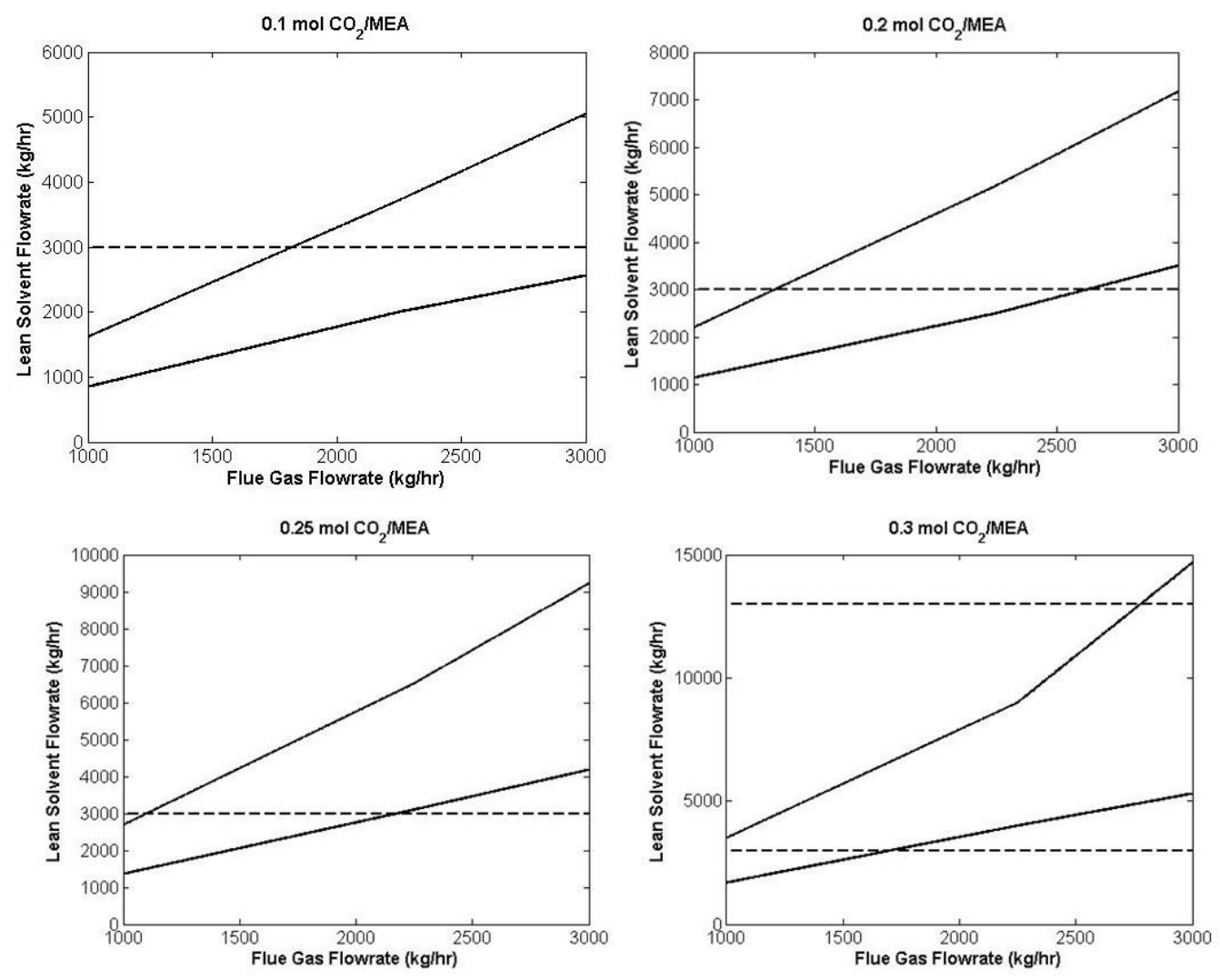

Figure 7-6. Estimated ranges of liquid flowrate, for a given value of lean loading and 15 wt\% $\mathrm{CO}_{2}$ in flue gas, for which $\mathrm{CO}_{2}$ capture percentage is constrained between $50-95 \%$. Solid lines represent the ranges of liquid flowrate for which requirement is met, and dashed line is included to illustrate additional constraint that liquid flowrate remains between $3000-13000 \mathrm{~kg} / \mathrm{hr}$. 

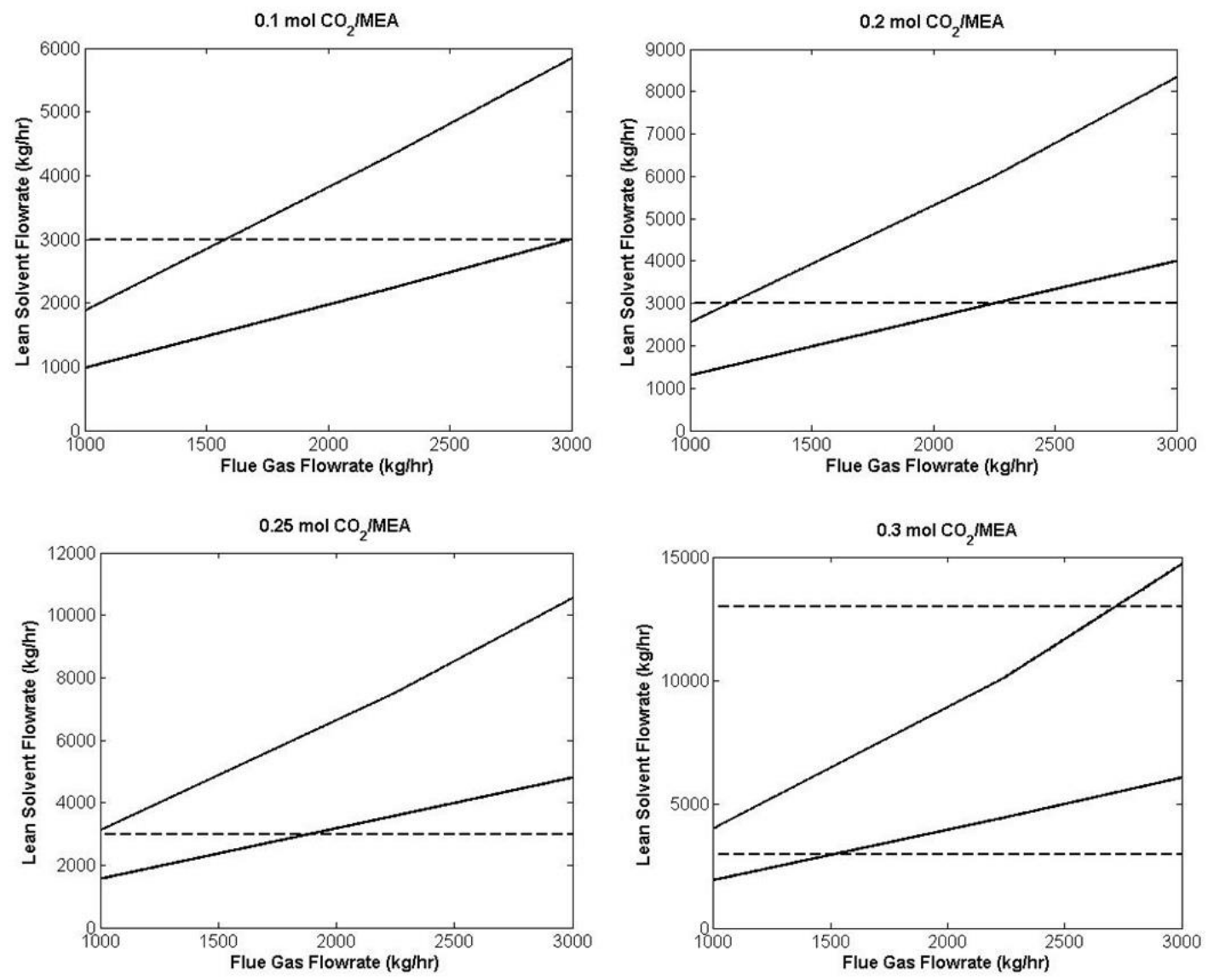

Figure 7-7. Estimated ranges of liquid flowrate, for a given value of lean loading and 17.5 wt\% $\mathrm{CO}_{2}$ in flue gas, for which $\mathrm{CO}_{2}$ capture percentage is constrained between $50-95 \%$. Solid lines represent the ranges of liquid flowrate for which requirement is met, and dashed line is included to illustrate additional constraint that liquid flowrate remains between $3000-13000 \mathrm{~kg} / \mathrm{hr}$.

As shown in Figures 7.5-7, the width of the range of lean solvent flowrate for which operation of the absorber column is considered desirable varies greatly with the other input variables. For example, at a low $\mathrm{CO}_{2}$ loading in solvent $\left(\sim 0.1 \mathrm{~mol} \mathrm{CO}_{2} / \mathrm{MEA}\right)$ and $\mathrm{CO}_{2}$ weight percentage in flue gas $(\sim 12.5 \%)$, no cases would be chosen for a low flue gas flowrate $(<1700 \mathrm{~kg} / \mathrm{hr})$, because the entire solvent range for which the column operates between $50-95 \%$ efficiency lies below the minimum of $3000 \mathrm{~kg} / \mathrm{hr}$. On the other hand, a 95\% capture condition would not be included for a high value of loading ( $0.3 \mathrm{~mol} \mathrm{CO} / \mathrm{MEA}), \mathrm{CO}_{2}$ weight percentage ( 17.5\%), and flue gas flowrate $(\sim 3000 \mathrm{~kg} / \mathrm{hr})$, because a solvent flowrate that is higher than the maximum of 13000 $\mathrm{kg} / \mathrm{hr}$ would be required. A similar, although less rigorous, procedure is used for the alternate process configurations, based on varying the number of absorber beds and the presence of 
intercooling, since some runs for these configurations are also desired to be included in the test run. It is also difficult to be considered in the design procedure since the decision variables are integer variables. Tabulated values of the liquid flow rate are given for the discrete values of flue gas flowrate and $\mathrm{CO}_{2}$ weight fraction and $\mathrm{CO}_{2}$ loading in the solvent in Appendix E.

\subsubsection{Stripper Column}

Although the design of the NCCC test plan is concerned primarily with the operation of the absorber column, some sensitivity analysis must also be performed for the stripper column. Although the lean solvent loading is considered as one of the input variables when designing the set of test runs, it is not directly manipulated as one of the plant variables, but it is dependent on the input of steam flowrate to the reboiler in the stripper. The steam flowrate is directly proportional to the reboiler duty, which is considered here as a simulation input. The lean solvent loading may primarily be considered a function of the reboiler duty $(\dot{Q})$, rich solvent loading $\left(\alpha_{\text {rich }}\right)$, and rich solvent flowrate $\left(\dot{m}_{\text {rich }}\right)$, of which the latter two are calculated as outputs of the absorber model. A sensitivity study for the required reboiler duty as a function of these input variables is given in Figure 7.8. As for the absorber model, the nominal MEA weight fraction in the solvent is set at $30 \%$. The solvent temperature and operating pressure are set at typical values of $100^{\circ} \mathrm{C}$ and $180 \mathrm{kPa}$, respectively. 

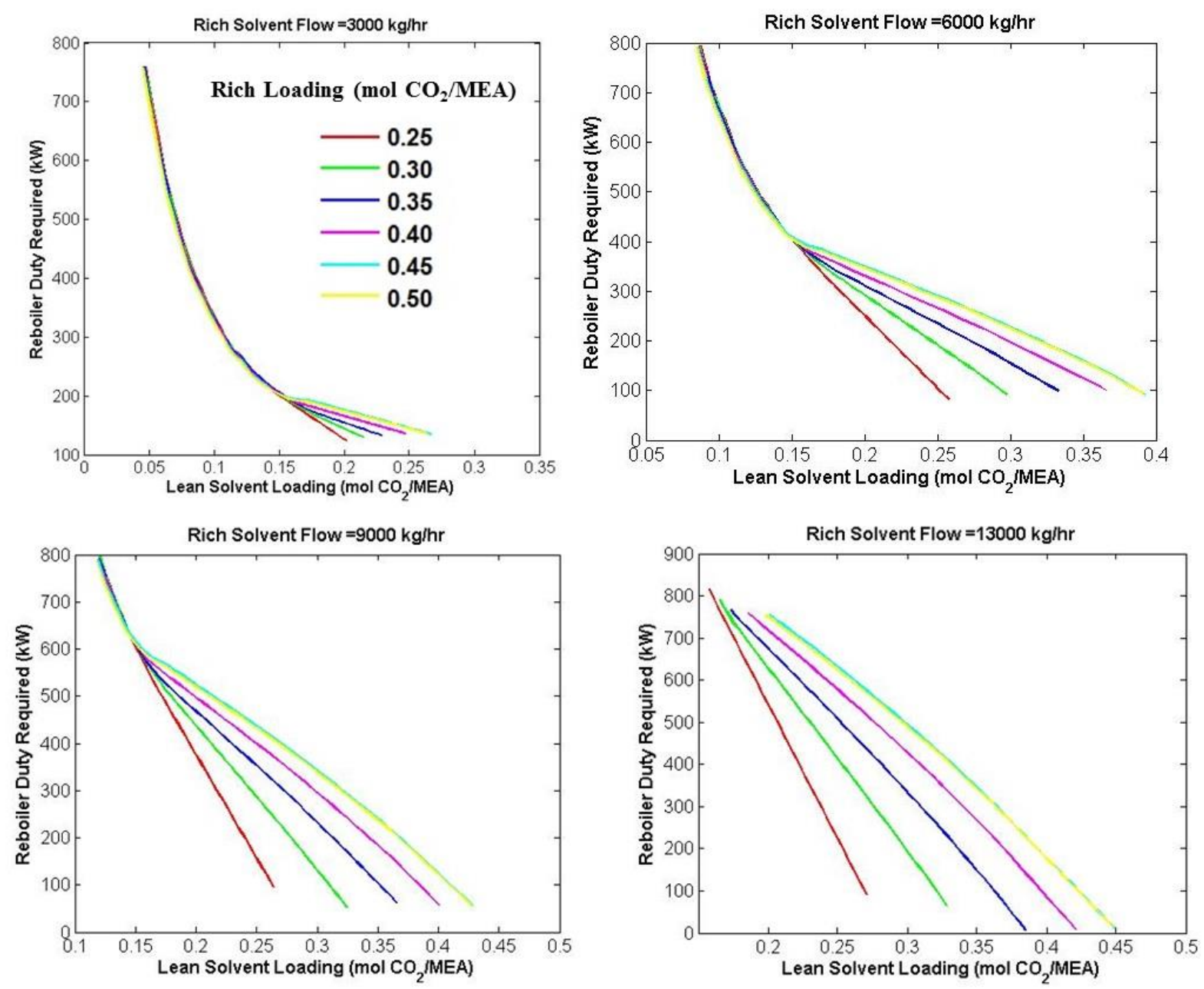

Figure 7-8. Sensitivity study in which the required reboiler duty to reduce the lean solvent loading to a given value is calculated as a function of rich solvent flowrate and $\mathrm{CO}_{2}$ loading.

The reboiler duty requirement increases with increasing solvent flowrate and rich loading. As the outlet lean solvent loading approaches zero, the required reboiler duty becomes infinitely large, making operation at very low lean loading infeasible due to high operating costs associated with steam input requirement. It is shown that for a lean loading lower than a certain value $(\sim 0.15$ mol $\mathrm{CO}_{2} / \mathrm{MEA}$ ), the reboiler duty requirement does not continue to decrease with a decrease in the rich solvent loading.

\subsection{Confidence Interval Calculation}

The distributions of the 13 parameters described in Chapter 6 have been propagated through the absorber model over a range of input variables of interest. Figure 7.9 shows estimates of the $95 \%$ 
confidence intervals for the absorber efficiency, calculated from estimations of the cumulative density functions (cdfs) of the values obtained from propagating the uncertainty through the model.
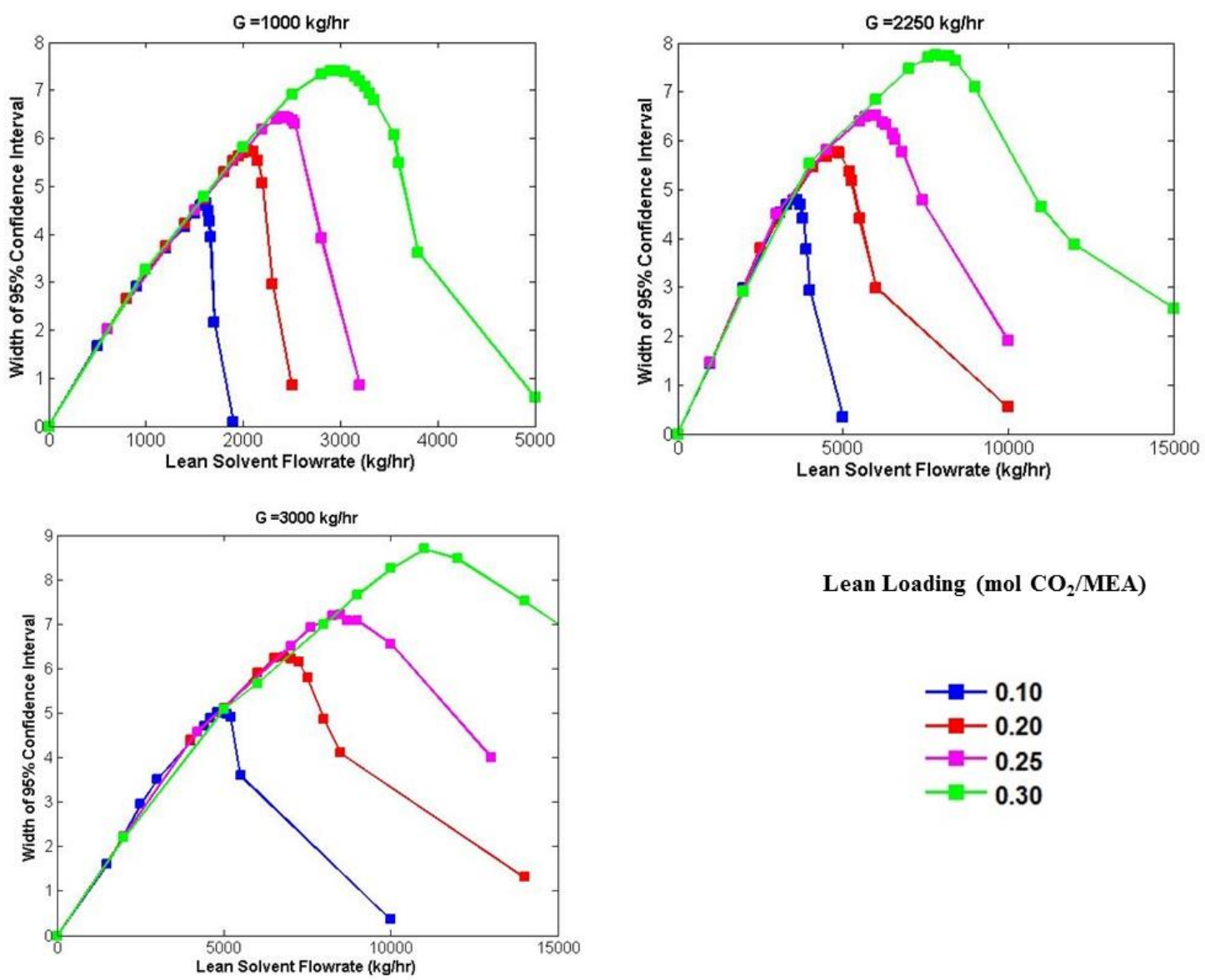

Lean Loading (mol $\left.\mathrm{CO}_{2} / \mathrm{MEA}\right)$

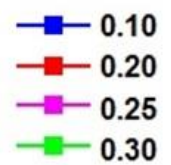

Figure 7-9. Estimated widths of $95 \%$ confidence intervals in absorber efficiency as a function of lean solvent flowrate, flue gas flowrate, and lean loading. Comparison given for a fixed $\mathrm{CO}_{2}$ weight percentage in flue gas $(15 \%)$. Squares represent discrete points for which the confidence intervals are calculated, and the lines are included to improve visibility of the trends.

With all other variables held constant, the amount of uncertainty in the $\mathrm{CO}_{2}$ capture percentage is shown to increase approximately linearly with increasing lean solvent flowrate until reaching a maximum value, and decreases towards zero with further increase in the solvent flowrate. The maximum value of the confidence interval width, and the solvent flowrate at which it occurs, increases with increasing solvent flowrate. Similar trends may be shown for variable $\mathrm{CO}_{2}$ weight 
percentage in the flue gas. These trends are shown from another perspective in Figure 7.10, in which the confidence interval widths are given as a function of the $\mathrm{CO}_{2}$ capture percentage instead of the lean solvent flowrate.
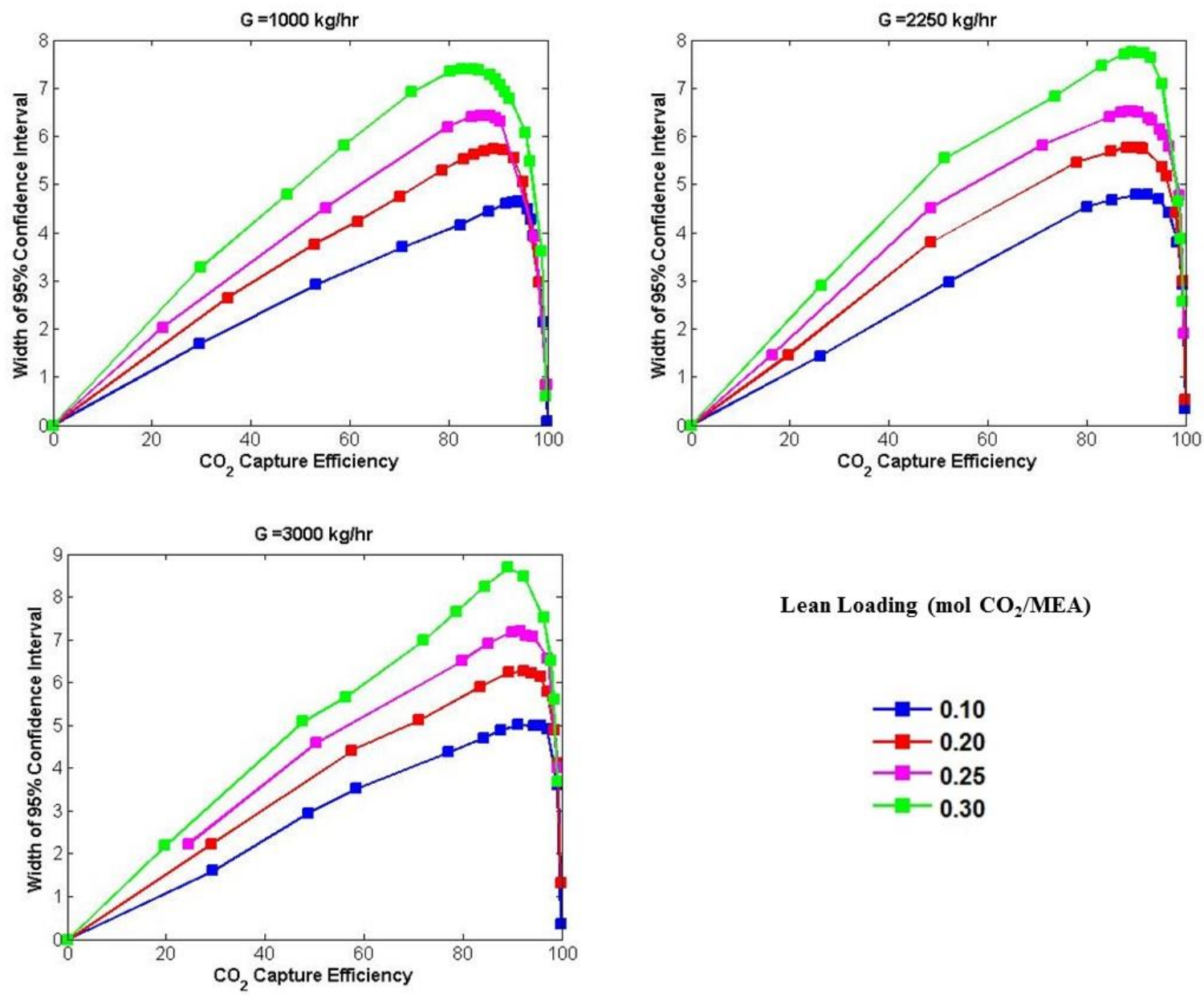

Lean Loading (mol $\left.\mathrm{CO}_{2} / \mathrm{MEA}\right)$

Figure 7-10. Estimated widths of $95 \%$ confidence intervals in absorber efficiency as a function of $\mathrm{CO}_{2}$ capture percentage, flue gas flowrate, and lean loading. Comparison given for a fixed $\mathrm{CO}_{2}$ weight percentage in flue gas $(15 \%)$. Squares represent discrete points for which the confidence intervals are calculated, and the lines are included to improve visibility of the trends.

From Figure 7.10, it becomes clear that the decrease in the $\mathrm{CO}_{2}$ capture percentage with further increase in lean solvent flowrate occurs at the point in which the column transitions between a reaction-limited regime and a mass transfer-limited regime. This is consistent with the earlier 
result in which the estimated uncertainty in the absorber efficiency was shown to be very small for operation near complete capture.

\subsection{Design of Experiments}

In the design of experiments methodology, the $\mathrm{CO}_{2}$ capture percentage of the absorber column is represented by a surrogate model, which may be denoted as:

$$
\hat{y}=\hat{y}\left(\tilde{x}, \tilde{\theta}_{1}, \tilde{\theta}_{2}\right)
$$

The set of independent variables, which is defined in Eq. 7.1a-d, is denoted as $\tilde{x}$, and $\hat{y}$ refers to the response surface model prediction of the $\mathrm{CO}_{2}$ capture percentage. The model parameters are divided into two groups; $\tilde{\theta}_{1}$ refers to the set of parameters of fixed uncertainty and $\tilde{\theta}_{2}$ refers to the set of parameters for which the distributions are updated in this work in light of the process data. The major rationale for considering two groups of parameters for this analysis is that the uncertainty of the physical property model parameters $\left(\tilde{\theta}_{1}\right)$ have been adequately estimated from corresponding property data, and their uncertainty is independent of plant hardware. The distributions of the parameters for the mass transfer and hydraulics models $\left(\tilde{\theta}_{2}\right)$, however, have been calibrated in previous work for bench scale data that were not collected specifically for the packing type (MellapakPlus ${ }^{\mathrm{TM}} 252 \mathrm{Y}$ ). Accordingly, the distributions of these parameters may be adjusted upon considering the process level data in a Bayesian framework. The response surface model is developed by simultaneously sampling from the parameter distributions (for both $\tilde{\theta}_{1}$ and $\tilde{\theta}_{2}$ ) and from the input variable space $\tilde{x}$. Due to some slight discrepancy in the planned test runs and the actual experimental data collected, the ranges of the ranges of the $\mathrm{CO}_{2}$ loading and the $\mathrm{CO}_{2}$ weight fraction have been modified to encompass all experimental data. Essentially, Eqns. 7.1a-d are replaced with:

$$
\begin{gathered}
L \in[3000-13000] \mathrm{kg} / \mathrm{hr} \\
G \in[1000-3000] \mathrm{kg} / \mathrm{hr} \\
\alpha_{\text {lean }} \in[0.1-0.35] \mathrm{mol} \mathrm{CO}_{2} / \mathrm{MEA} \\
w_{\mathrm{CO}_{2}} \in[0.1-0.175]
\end{gathered}
$$


For sampling the variables contained in $\tilde{x}$, a sample is taken from the uniform distribution of each of the four variables. Only points for which the value of $L$ lies within the estimated ranges corresponding to a $\mathrm{CO}_{2}$ capture percentage range of $50-95 \%$ are incorporated into the development of the response surface model. The estimated ranges for the liquid flowrate are determined by using the trilinear interpolation algorithm, as described previously in Section 7.2.1, which has been modified to be fully inclusive of the modified variable ranges in Eq. 7.3cd. A total of 5773 observations are used for developing the response surface model, and the MARS method is used as described previously. A parity plot is given in Figure 7.11 to show the quality of the response surface as a surrogate for the actual model.

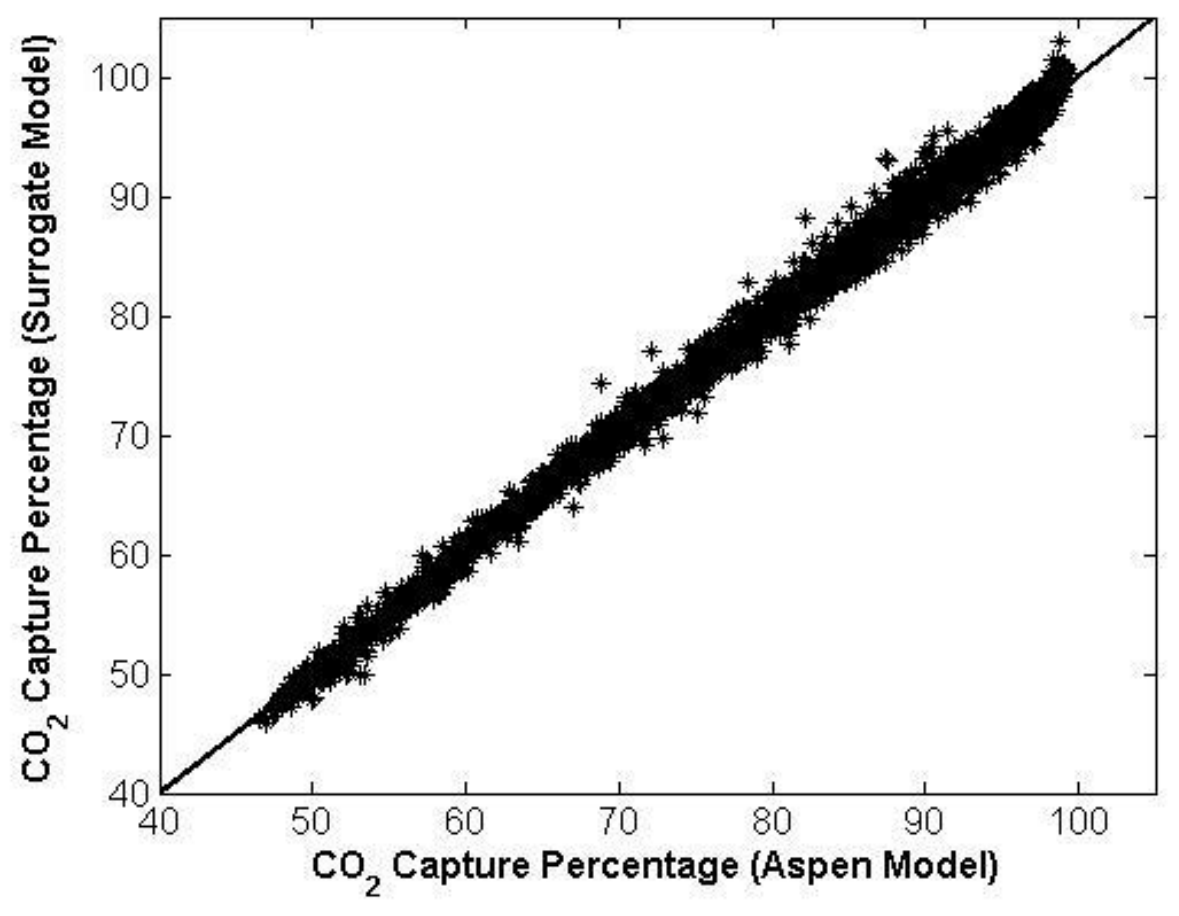

Figure 7-11. Parity plot for comparison of $\mathrm{CO}_{2}$ capture percentage predicted by original Aspen Plus model and surrogate response surface model.

The response surface model developed using MARS has been shown to be an adequate surrogate for the actual absorber process model, and the correlation between the two models has been calculated as $R^{2} \approx 0.995$.

The test cases have been chosen by a research collaborator, using an algorithm that involves a space-filling design while attempting to select points for which the confidence intervals, which have been estimated as described in Section 7.3, are relatively wide. The test runs are selected 
from a grid of approximately 450 points which covers the input variable values over the ranges described in Eq. 7.1, with the estimated $\mathrm{CO}_{2}$ capture percentage constrained between 50-95\% using the information presented in Figures 7.5-7. The final test plan, which includes 20 cases, is presented in Table 7.1.

Table 7-1. First set of cases selected for 2017 MEA test campaign at NCCC

\begin{tabular}{ccccccc}
\hline $\begin{array}{c}\text { Case } \\
\text { No. }\end{array}$ & $\begin{array}{c}\text { Lean } \\
\text { Solvent } \\
\text { Flowrate } \\
(\mathrm{kg} / \mathrm{hr})\end{array}$ & $\begin{array}{c}\text { Flue Gas } \\
\text { Flowrate } \\
(\mathrm{kg} / \mathrm{hr})\end{array}$ & $\begin{array}{c}\text { Lean } \\
\text { Loading }\end{array}$ & $\begin{array}{c}\text { Reboiler } \\
\text { Steam } \\
\text { Flowrate } \\
(\mathrm{kg} / \mathrm{hr})\end{array}$ & $\begin{array}{c}\text { Flue Gas } \\
\mathrm{CO}_{2} \\
\text { Weight } \\
\text { Fraction }\end{array}$ & $\begin{array}{c}\mathrm{CO}_{2} \text { Capture } \\
\text { Percentage } \\
\text { (Model } \\
\text { Prediction) }\end{array}$ \\
\hline 1 & 3911 & 1250 & 0.3 & 251 & 0.175 & 77.3 \\
2 & 3200 & 2250 & 0.25 & 261 & 0.14 & 54.8 \\
3 & 3800 & 2500 & 0.15 & 438 & 0.1583 & 72.9 \\
4 & 9384 & 3000 & 0.25 & 781 & 0.175 & 89.3 \\
5 & 4171 & 3000 & 0.1 & 772 & 0.175 & 69.6 \\
6 & 6817 & 2250 & 0.3 & 438 & 0.175 & 72.8 \\
7 & 8186 & 3000 & 0.25 & 688 & 0.125 & 96.1 \\
8 & 3133 & 1750 & 0.3 & 200 & 0.125 & 61.0 \\
9 & 7946 & 3000 & 0.2 & 806 & 0.1583 & 97.3 \\
10 & 3017 & 2750 & 0.1 & 558 & 0.1583 & 60.8 \\
11 & 6514 & 2500 & 0.25 & 536 & 0.175 & 78.6 \\
12 & 3609 & 3000 & 0.15 & 418 & 0.125 & 71.8 \\
13 & 8024 & 2500 & 0.25 & 674 & 0.1583 & 96.3 \\
14 & 9384 & 3000 & 0.25 & 781 & 0.175 & 89.3 \\
15 & 3230 & 2250 & 0.1 & 597 & 0.175 & 72.3 \\
16 & 6932 & 2750 & 0.2 & 692 & 0.175 & 90.2 \\
17 & 4341 & 2000 & 0.2 & 430 & 0.1583 & 87.7 \\
18 & 3360 & 1500 & 0.2 & 331 & 0.175 & 83.7 \\
19 & 3370 & 2750 & 0.15 & 388 & 0.175 & 53.9 \\
20 & 4734 & 2250 & 0.15 & 550 & 0.175 & 90.6 \\
\hline
\end{tabular}

The estimated values of $\mathrm{CO}_{2}$ capture given in Table 7.1 are calculated from the actual model instead of an approximation, so some of the values may be slightly above $95 \%$. The final test plan for the first 20 runs is also displayed graphically in Figure 7.12, and compared to the tests conducted during the 2014 test campaign. 


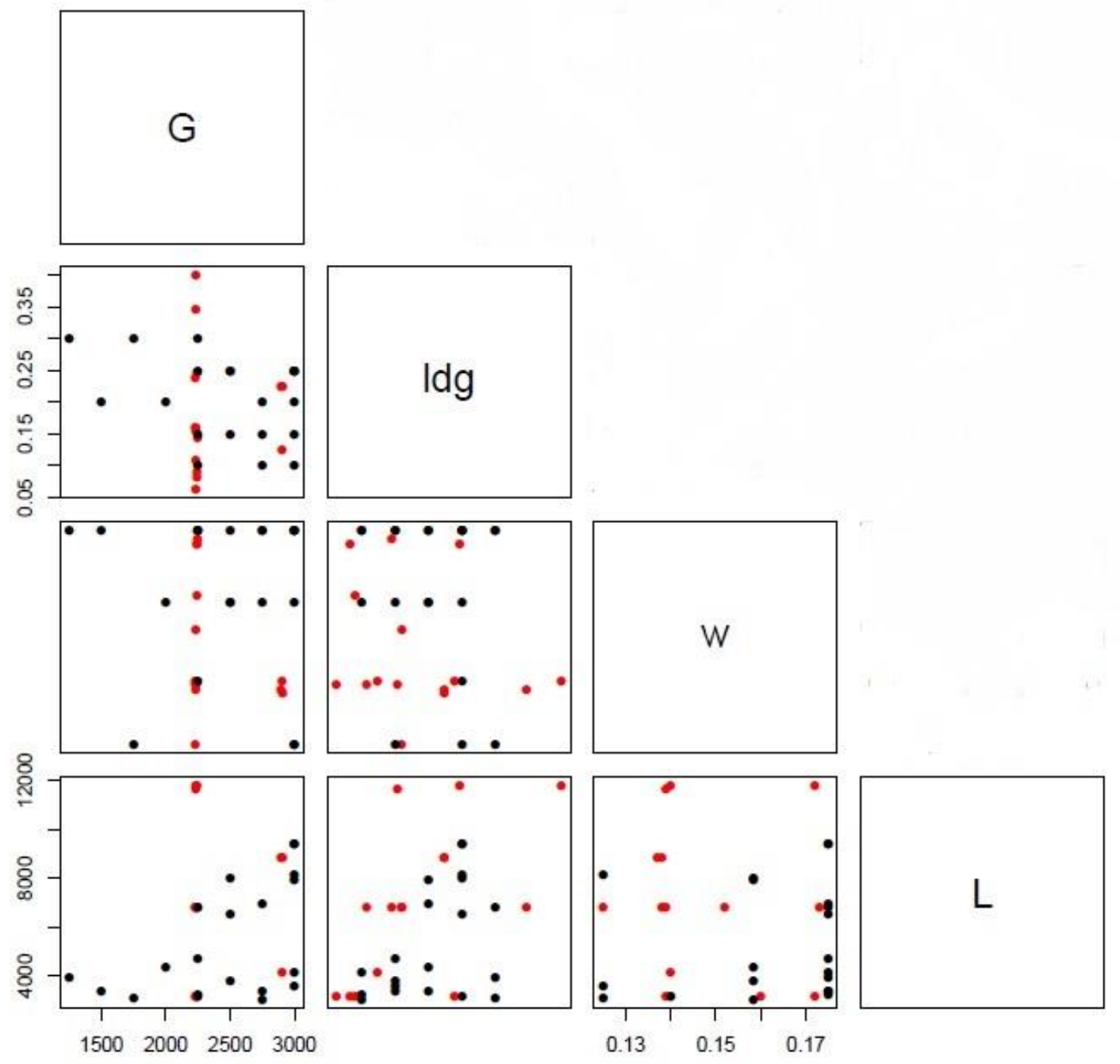

Figure 7-12. Graphical comparison of first set of experiments for 2017 campaign (black dots) and 2014 campaign (red dots)

Figure 7.12 shows the test points spread throughout the four dimensional input space. The input variables and their planned ranges are defined in Eq. 7.1a-d, and the variables labeled as 'ldg' and ' $\mathrm{w}$ ' correspond to $\alpha_{\text {lean }}$ and $w_{\mathrm{CO}_{2}}$, respectively. It should also be noted that only the data for the three beds with intercooling configuration, which comprise 15 of the 23 data points, are represented in this figure.

The final set of data incorporated into the first round of the parameter distribution updating is summarized in Table 7.2. The experimental data for $\mathrm{CO}_{2}$ capture percentage are compared with model predictions, and the comparison is also given in Figure 7.13 
Table 7-2. Results of three beds with intercooling test for first test plan

\begin{tabular}{|c|c|c|c|c|c|c|}
\hline \multirow[t]{2}{*}{ Data No. } & \multirow{2}{*}{$\begin{array}{c}\text { Lean } \\
\text { Solvent } \\
\text { Flowrate } \\
(\mathrm{kg} / \mathrm{hr})\end{array}$} & \multirow{2}{*}{$\begin{array}{c}\text { Flue Gas } \\
\text { Flowrate } \\
(\mathrm{kg} / \mathrm{hr})\end{array}$} & \multirow{2}{*}{$\begin{array}{c}\text { Lean } \\
\text { Solvent } \\
\text { Loading } \\
(\text { mol } \\
\left.\mathrm{CO}_{2} / \mathrm{MEA}\right)\end{array}$} & \multirow{2}{*}{$\begin{array}{l}\text { Flue Gas } \\
\mathrm{CO}_{2} \\
\text { Fraction } \\
\text { (weight) }\end{array}$} & \multicolumn{2}{|c|}{$\mathrm{CO}_{2}$ Capture Percentage } \\
\hline & & & & & Data & Model \\
\hline 1 & 8180 & 3000 & 0.242 & 0.125 & 97.5 & 97.2 \\
\hline 2 & 7130 & 2690 & 0.245 & 0.150 & 93.4 & 90.2 \\
\hline 3 & 3354 & 1500 & 0.243 & 0.162 & 79.7 & 77.0 \\
\hline 4 & 3600 & 3000 & 0.192 & 0.117 & 70.6 & 66.6 \\
\hline 5 & 3380 & 2750 & 0.2 & 0.160 & 53.8 & 50.2 \\
\hline 6 & 3130 & 1750 & 0.314 & 0.116 & 51.7 & 60.6 \\
\hline 7 & 4730 & 2255 & 0.234 & 0.164 & 72.5 & 73.0 \\
\hline 8 & 3230 & 2240 & 0.237 & 0.160 & 56.3 & 51.8 \\
\hline 9 & 3224 & 2245 & 0.135 & 0.162 & 74.2 & 72.9 \\
\hline 10 & 7980 & 2492 & 0.315 & 0.163 & 79.9 & 74.2 \\
\hline 11 & 3016 & 2761 & 0.16 & 0.145 & 60.5 & 55.7 \\
\hline 12 & 4170 & 2920 & 0.14 & 0.160 & 76.0 & 72.5 \\
\hline 13 & 6910 & 2680 & 0.255 & 0.162 & 80.6 & 80.9 \\
\hline 14 & 6505 & 2500 & 0.314 & 0.162 & 57.8 & 63.1 \\
\hline 15 & 8000 & 2494 & 0.315 & 0.162 & 76.8 & 74.6 \\
\hline
\end{tabular}




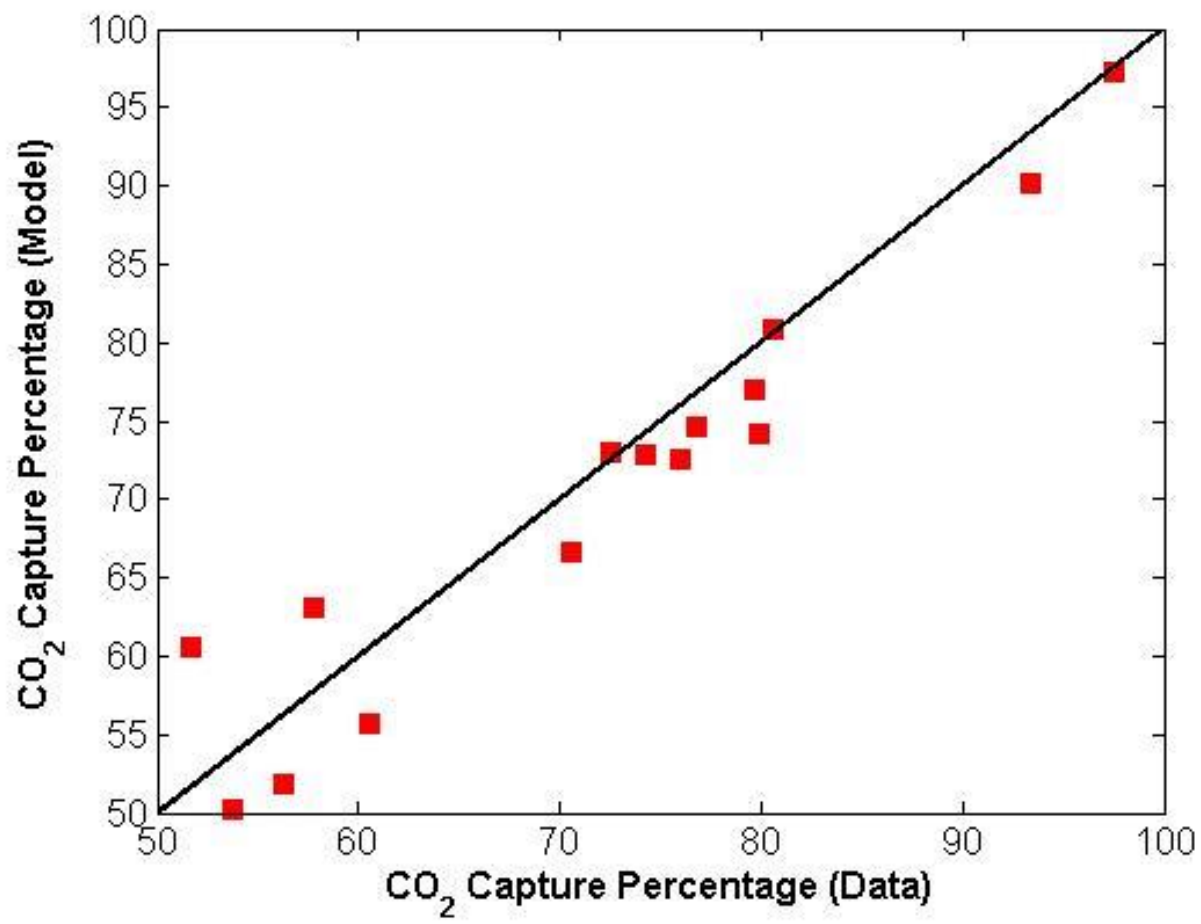

Figure 7-13. Parity plot for comparison of model prediction of $\mathrm{CO}_{2}$ capture percentage to experimental data, for operation with three beds and intercooling.

These data are incorporated into a Bayesian inference methodology, which is similar to that described in Chapter 2 for the process submodels, with the exception that some of the parameters are considered to be of fixed uncertainty. A sample from the parameters of fixed uncertainty $\left(\tilde{\theta}_{1}^{(1)}, \tilde{\theta}_{1}^{(2)}, \ldots, \tilde{\theta}_{1}^{(j)}, \ldots, \tilde{\theta}_{1}^{(n)}\right)$ of size $n$ is taken, and for each sample a posterior distribution of the parameters is $\tilde{\theta}_{2}$ is generated, which may be denoted as $\pi_{j}\left(\tilde{\theta}_{2} \mid Z, \tilde{\theta}_{1}^{(j)}\right)$. The final posterior distribution $\pi\left(\tilde{\theta}_{2} \mid Z\right)$ is taken by combining all $n$ of these distributions. A total of $n=100$ iterations is used for this work. The change in the distributions of the four parameters contained in $\tilde{\theta}_{2}$ is shown in Figure 7.14. The parameter numbers were previously defined in Tables 6.7-8. 

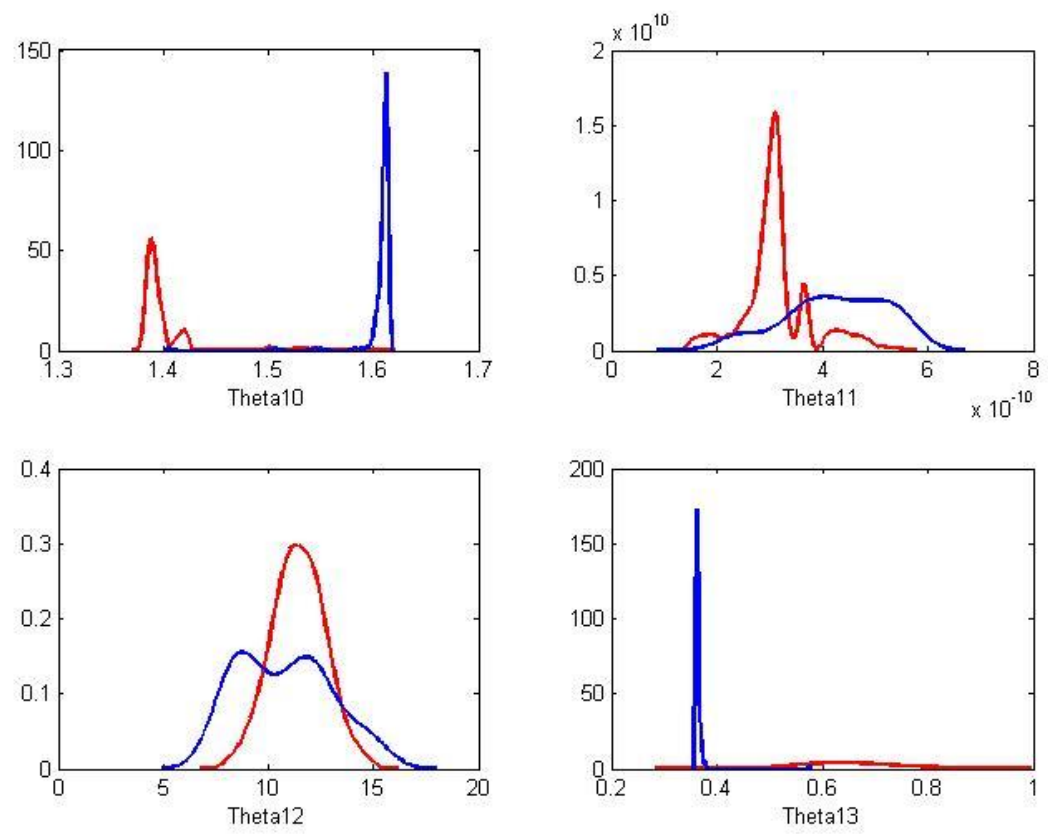

Figure 7-14. Estimated marginal pdfs for prior (red) and posterior (blue) distributions of parameters updated in Bayesian inference with $\mathrm{CO}_{2}$ capture percentage data

The values of parameters 10 and 13 are shifted as a result of the Bayesian inference, which may be attributed to the fact that the original values did not give the best fit of the model to the experimental data. The updated parameter distributions are used to determine the effect of incorporating the NCCC data into a Bayesian inference methodology to predict the updated model uncertainty, which is shown in Figure 7.15. 

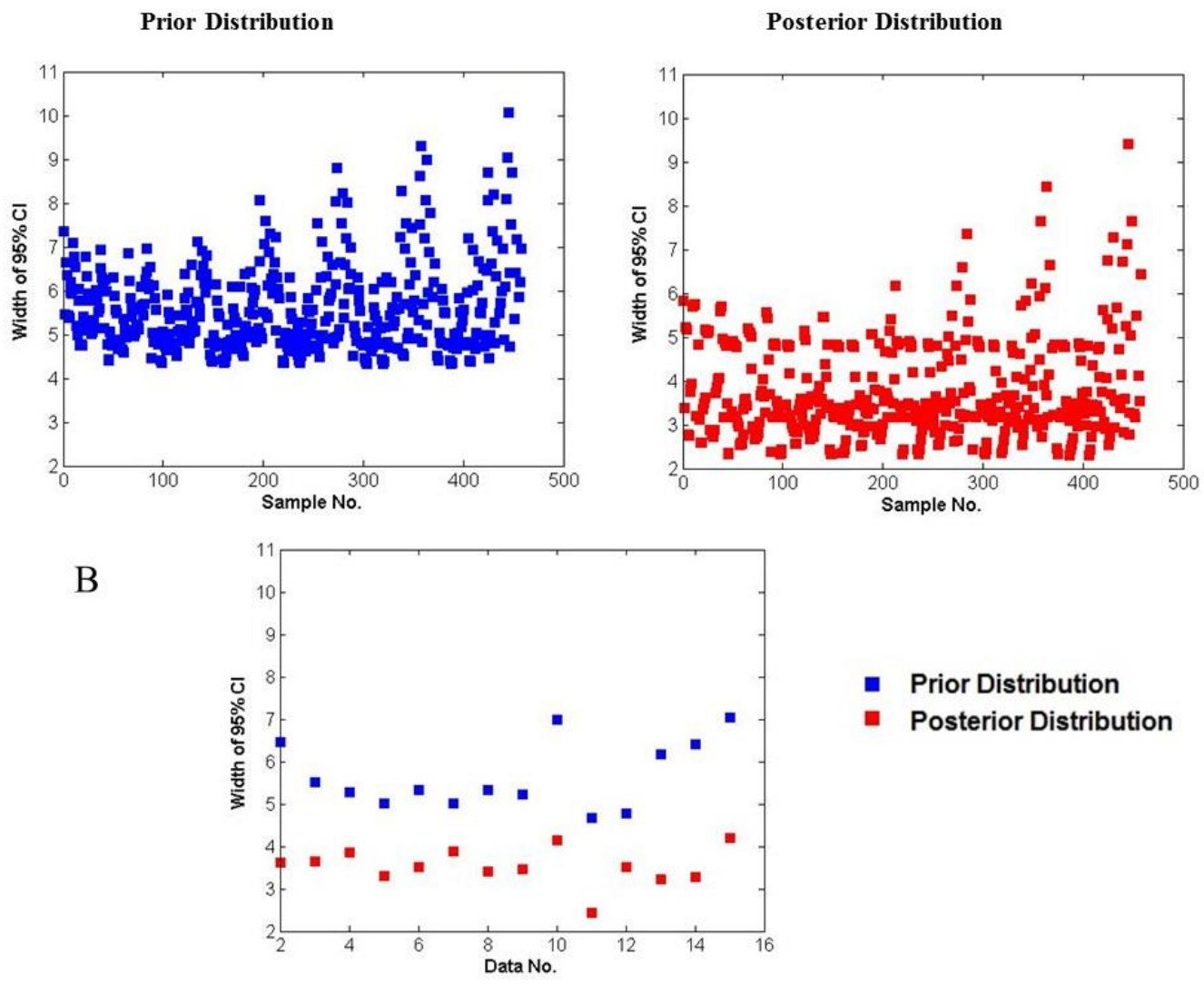

- Prior Distribution

- Posterior Distribution

Figure 7-15. Effect of incorporating absorber efficiency data into Bayesian estimation of mass transfer and hydraulics model parameters. Confidence interval widths, as calculated by the surrogate absorber model, are shown for (A) grid of 448 points spread throughout input space and $(B)$ points for which experimental data are collected.

Note that the first data point is not included in Figure $7.15 \mathrm{~b}$ due to the width of the confidence interval expanding due to inaccuracies in the response surface model at high $\mathrm{CO}_{2}$ capture percentage values. Similarly, other points (11 out of 459 points considered) are not included in Figure $7.15 \mathrm{a}$ due to the same issue. As a result of incorporating the experimental data, the width of the confidence interval decreases by an average of $1.80 \pm 0.65$ for all of the points considered in the grid (shown in Figure 7.15a) and by an average of $2.12 \pm 0.68$ for the points for which data were collected (shown in Figure 7.15b). This highlights the effectiveness of the new experimental data, collected for test cases chosen from the Bayesian DOE, in reducing the uncertainty in the stochastic model prediction of $\mathrm{CO}_{2}$ capture percentage. With the updated uncertainty values of $\mathrm{CO}_{2}$ capture from the grid of 450 points, a new test run is designed and 
presented in Table 7.3. Note that only three points are included due to time constraints encountered during the execution of this project.

Table 7-3. Test plan for second round of sequential DOE

\begin{tabular}{ccccccc}
\hline $\begin{array}{c}\text { Case } \\
\text { No. }\end{array}$ & $\begin{array}{c}\text { Lean } \\
\text { Solvent } \\
\text { Flowrate } \\
(\mathrm{kg} / \mathrm{hr})\end{array}$ & $\begin{array}{c}\text { Flue Gas } \\
\text { Flowrate } \\
(\mathrm{kg} / \mathrm{hr})\end{array}$ & $\begin{array}{c}\text { Lean } \\
\text { Loading } \\
(\mathrm{mol} \\
\left.\mathrm{CO}_{2} / \mathrm{MEA}\right)\end{array}$ & $\begin{array}{c}\text { Reboiler } \\
\text { Steam } \\
\text { Flowrate } \\
(\mathrm{kg} / \mathrm{hr})\end{array}$ & $\begin{array}{c}\text { Flue Gas } \\
\mathrm{CO}_{2} \text { Weight } \\
\text { Fraction }\end{array}$ & $\begin{array}{c}\mathrm{CO}_{2} \text { Capture } \\
\text { Percentage } \\
\text { (Model } \\
\text { Prediction) }\end{array}$ \\
\hline 1 & 7971 & 2500 & 0.3 & 520 & 0.125 & 91.62 \\
2 & 9881 & 2750 & 0.3 & 645 & 0.1417 & 91.28 \\
3 & 11675 & 2750 & 0.3 & 761 & 0.175 & 90.76 \\
\hline
\end{tabular}

For the updated test plan, it should be noted that all of the data are located in a regime close to complete $\mathrm{CO}_{2}$ capture, which is likely a result of relatively high uncertainty in this region. The resulting data are given in Table 7.4. Note that data values of the $\mathrm{CO}_{2}$ weight fraction were generally lower than the values given in the test plan, leading to higher values of $\mathrm{CO}_{2}$ capture percentage than expected.

Table 7-4. Experimental data for second round of sequential DOE

\begin{tabular}{cccccc}
\hline Data No. & $\begin{array}{c}\text { Lean Solvent } \\
\text { Flowrate } \\
(\mathrm{kg} / \mathrm{hr})\end{array}$ & $\begin{array}{c}\text { Flue Gas } \\
\text { Flowrate } \\
(\mathrm{kg} / \mathrm{hr})\end{array}$ & $\begin{array}{c}\text { Lean Loading } \\
(\text { mol } \\
\left.\mathrm{CO}_{2} / \mathrm{MEA}\right)\end{array}$ & $\begin{array}{c}\text { Flue Gas } \mathrm{CO}_{2} \\
\text { Weight } \\
\text { Fraction }\end{array}$ & $\begin{array}{c}\mathrm{CO}_{2} \text { Capture } \\
\text { Percentage } \\
\text { (Data) }\end{array}$ \\
\hline 1 & 7959 & 2497 & 0.3 & 0.118 & 96.1 \\
2 & 9871 & 2746 & 0.3 & 0.133 & 97.7 \\
3 & 11412 & 2748 & 0.3 & 0.162 & 94.9 \\
\hline
\end{tabular}

A final parity plot for all absorber data for the three beds with intercooling configuration from NCCC, including the 2014 campaign as well as both iterations of the 2017 campaign, is given in Figure 7.16. 


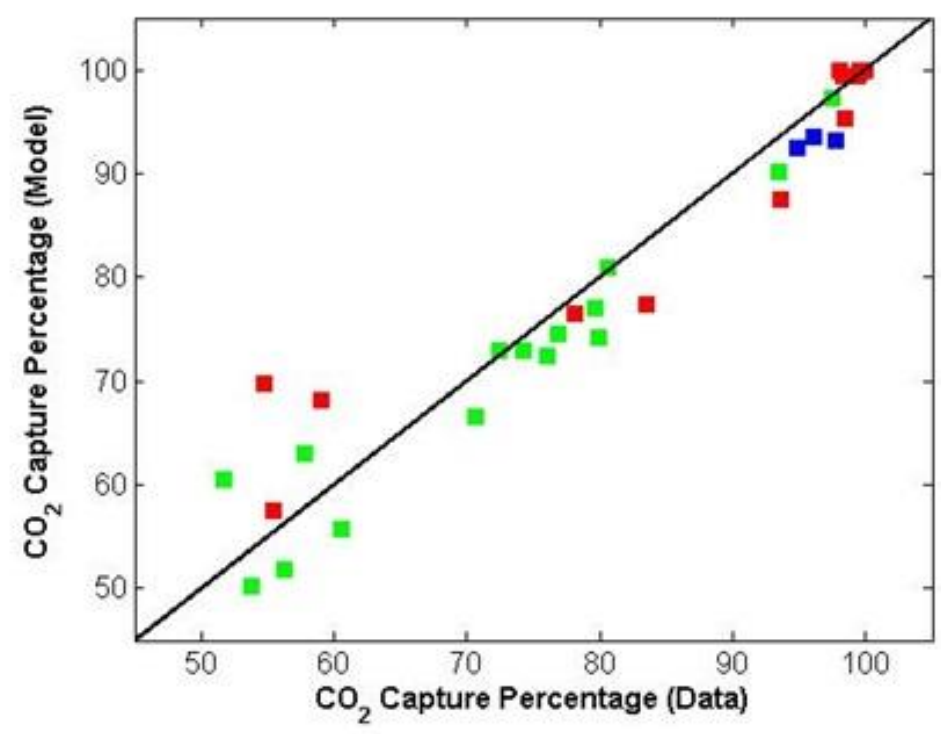

2014 Campaign

= 2017 Campaign (1st Round)

- 2017 Campaign (2nd Round)

Figure 7-16. Parity plot for $\mathrm{CO}_{2}$ capture percentage in absorber (3 beds with intercooling configuration) for complete set of NCCC data.

As shown in Figure 7.16, the data for the 2017 campaign are widely spread over the range of $\mathrm{CO}_{2}$ capture percentage of interest, especially in comparison to the 2014 campaign, in which many of the data are clustered at very high values of $\mathrm{CO}_{2}$ capture. For the second round of the 2017 campaign, the data are located in a region ( $~ 95-98 \% \mathrm{CO}_{2}$ capture) in which the estimated uncertainty, calculated by propagating the submodel parametric uncertainty through the process model, is relatively high. The data given in Table 7.4 are incorporated into a Bayesian inference framework, as described previously. The distribution of the thermodynamic model parameters remains constant, and the distribution of the mass transfer and hydraulic model parameters is updated, with the posterior given in Figure 7.14 used as the prior distribution for this round of UQ. The estimated single parameter marginal prior and posterior distributions for the mass transfer and hydraulics model parameters are given, for the second round of the Bayesian DOE, in Figure 7.17. 

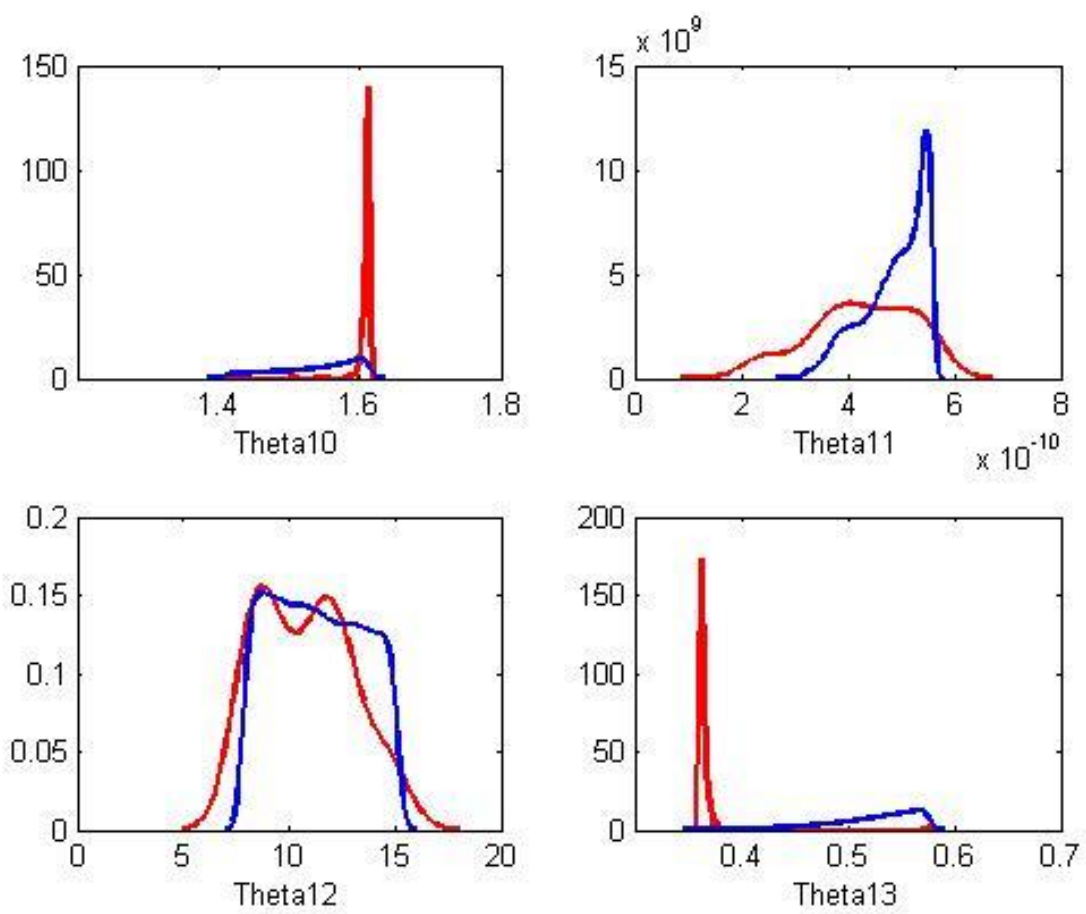

Figure 7-17. Estimated marginal pdfs for prior (red) and posterior (blue) distributions of parameters for second round of Bayesian DOE.

The corresponding change in the estimated values of the width of the $95 \%$ confidence intervals in $\mathrm{CO}_{2}$ capture percentage for the data points is given in Figure 7.18.

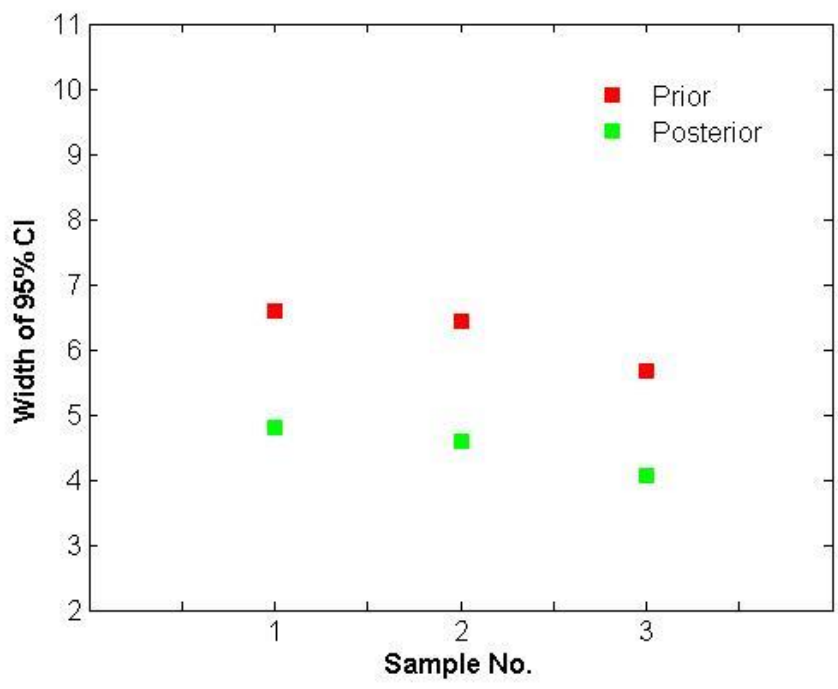

Figure 7-18. Change in estimated width of $95 \%$ confidence interval in $\mathrm{CO}_{2}$ capture percentage for points in which data were collected in second round of Bayesian DOE. 
The confidence intervals for the experimental conditions in which data were collected in the second round of Bayesian DOE are shown to decrease in width as a result of updating the parameter distributions. Due to time constraints, no further iterations of this process could be performed during the 2017 test campaign. Nevertheless, the applicability of the Bayesian DOE to the planning of a pilot plant test campaign has been demonstrated in this work.

\subsection{Alternative Process Configurations}

During the 2014 MEA campaign, very few data were obtained for absorber configurations other than three beds with intercooling, which may be considered as the default configuration. As these data are valuable for assessing the overall predictability of the absorber model, some effort has been made to include test runs other than those for the default configuration. Test runs with variable bed numbers are especially useful for determining the predictability with respect to the packing height of the absorber.

The test runs collected for cases in which the absorber is operated with one and two beds are summarized in Tables 7.5-6, respectively. No intercooling was used between the beds for the two bed operation in this case. These test runs have been selected from a space filling algorithm, similar to that used for the default configuration, although the confidence intervals obtained from propagating the parametric uncertainty are not taken into consideration here. The final set of data collected is similar to the test plan, with some variation in the input variable values. Note that some of the values of the composition variables fall slightly outside the ranges defined for the test plan (0.1-0.3 mol CO $2 / \mathrm{MEA}$ for the lean solvent loading and 0.125-0.175 for flue gas $\mathrm{CO}_{2}$ weight fraction), although this does not have a major impact on the quality of the data obtained. The quality of the model fit to the experimental data is also shown in Figure 7.19 in the form of a parity plot. 
Table 7-5. Results of one bed absorber test

\begin{tabular}{ccccccc}
\hline Data No. & $\begin{array}{c}\text { Lean } \\
\text { Solvent } \\
\text { Flowrate } \\
(\mathrm{kg} / \mathrm{hr})\end{array}$ & $\begin{array}{c}\text { Flue Gas } \\
\text { Flowrate } \\
(\mathrm{kg} / \mathrm{hr})\end{array}$ & $\begin{array}{c}\text { Lean } \\
\text { Solvent } \\
\text { Loading } \\
(\mathrm{mol} \\
\left.\mathrm{CO}_{2} / \mathrm{MEA}\right)\end{array}$ & $\begin{array}{c}\text { Flue } \mathrm{Gas} \\
\mathrm{CO}_{2} \\
\text { Fraction } \\
(\text { Weight })\end{array}$ & & $\mathrm{CO}_{2}$ Capture Percentage \\
\cline { 5 - 7 } & & & 0.15 & 0.118 & 97.1 & \\
\hline 1 & 6185 & 1997 & 0.20 & 0.118 & 92.3 & 87.6 \\
2 & 7765 & 2499 & 0.25 & 0.140 & 89.5 & 84.0 \\
3 & 7517 & 2013 & 0.25 & 87.6 \\
4 & 6160 & 1500 & 0.25 & 0.162 & 88.9 & 87.3 \\
5 & 5237 & 1498 & 0.26 & 0.118 & 86.4 & 58.8 \\
6 & 7665 & 2700 & 0.314 & 0.118 & 60.2 & 78.8 \\
7 & 5414 & 1000 & 0.34 & 0.150 & 76.4 & \\
\hline
\end{tabular}

Table 7-6. Results of two bed absorber test

\begin{tabular}{ccccccc}
\hline Data No. & $\begin{array}{c}\text { Lean } \\
\text { Solvent } \\
\text { Flowrate } \\
(\mathrm{kg} / \mathrm{hr})\end{array}$ & $\begin{array}{c}\text { Flue Gas } \\
\text { Flowrate } \\
(\mathrm{kg} / \mathrm{hr})\end{array}$ & $\begin{array}{c}\text { Lean } \\
\text { Solvent } \\
\text { Loading } \\
(\text { mol } \\
\left.\mathrm{CO}_{2} / \mathrm{MEA}\right)\end{array}$ & $\begin{array}{c}\text { Flue } \mathrm{Gas} \\
\mathrm{CO}_{2} \\
\text { Fraction } \\
(\text { weight })\end{array}$ & & $\mathrm{CO}_{2}$ Capture Percentage \\
\cline { 6 - 7 } & & & 0.3 & 0.15 & 77.8 & \\
\hline 1 & 4912 & 1500 & 0.2 & 0.175 & 80.5 & 81.2 \\
2 & 4600 & 2000 & 0.3 & 0.140 & 87.0 & 81.5 \\
3 & 9534 & 2502 & 0.2 & 0.120 & 96.4 & 96.9 \\
\hline
\end{tabular}




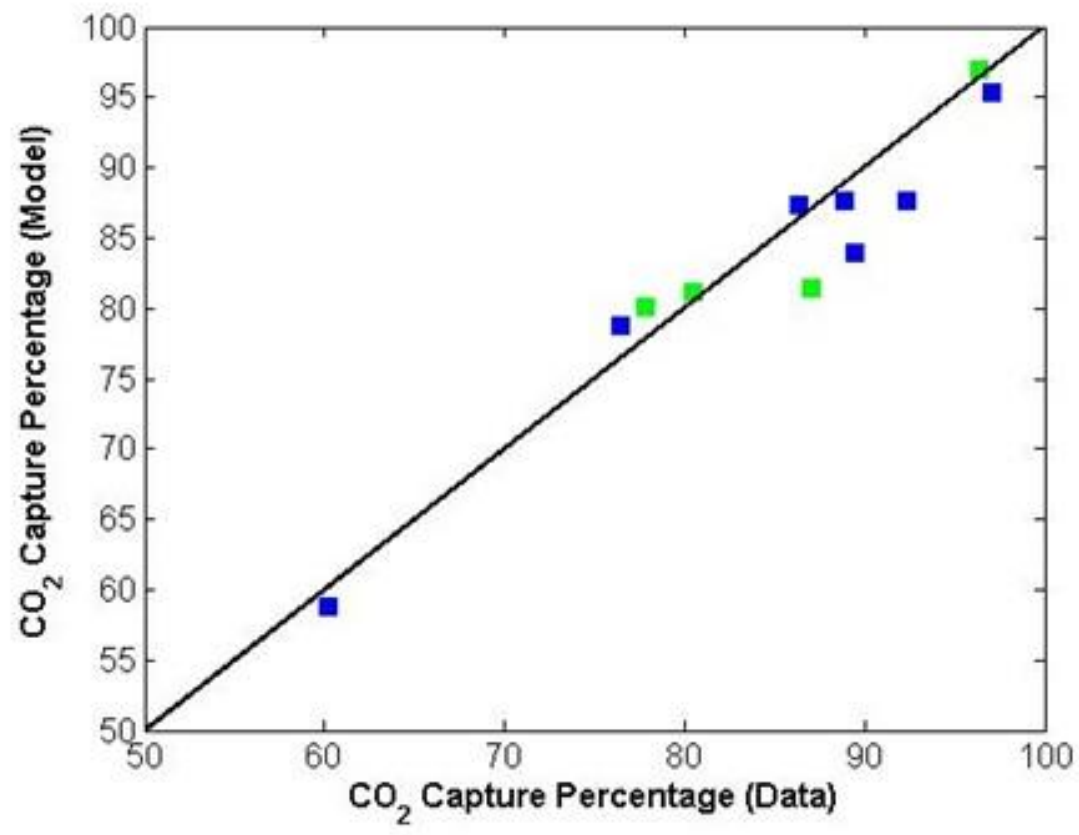

- One Bed Cases

nwo Bed Cases

Figure 7-19. Parity plot for $\mathrm{CO}_{2}$ capture percentage predicted by model and experimental data for cases in which absorber is operated with one or two beds

The fit of the model to experimental data for absorber operation with one or two beds is shown to be reasonably accurate. This provides further insight of the predictability of the CCSI MEA model for the absorber column with variation in the packing height, especially considering that few runs were conducted for these configurations in the 2014 campaign.

\subsection{Conclusions}

In conclusion, a sequential Bayesian DOE has been proposed in this work and applied to a MEA test campaign in the summer of 2017 at NCCC. The test campaign has been planned with the goal of constraining the $\mathrm{CO}_{2}$ capture percentage between $50-95 \%$, so as to complement the data obtained from the 2014 at the same plant, for $\mathrm{CO}_{2}$ capture percentage was above $99 \%$ for many of the points. The Bayesian DOE is employed to sequentially update the test plan in light of new experimental data as they are collected. This methodology incorporates an estimate of the uncertainty of the absorber efficiency as a function of the model inputs (solvent and gas stream flow rates and compositions) over their ranges of interest. The prior uncertainties are estimated 
by propagating the submodel uncertainties (the posterior distributions from submodel UQ) through the absorber model. The test runs are chosen by an algorithm that seeks to fill the input space while selecting points for which the estimated uncertainty is relatively high. As the new data are collected, they are implemented into a Bayesian inference procedure in which the parameter distributions of the mass transfer and hydraulics models are updated while the uncertainty in the thermodynamic model parameters is kept constant. The effectiveness of this procedure in reducing the uncertainty in the model has been demonstrated. Furthermore, additional data have been obtained for absorber operation with one and two beds, and the model has been shown to match the $\mathrm{CO}_{2}$ capture percentage accurately for these data. 


\section{Chapter 8: Conclusions and Future Work}

A major project of the Carbon Capture Simulation Initiative (CCSI) was the development of a rigorous process model for solvent-based $\mathrm{CO}_{2}$ capture using aqueous monoethanolamine as a baseline solvent. This model has been created from a rigorous approach in which submodels are developed using appropriate bench-scale data with quantified parametric uncertainty. The submodels are incorporated into an overall process simulation in Aspen Plus ${ }^{\circledR}$, and this model has been validated with steady-state pilot plant data from NCCC, which is a larger pilot scale system than most for which data are available in the open literature. The model has been shown to fit absorber and stripper performance data accurately without tuning any additional parameters to improve the fit to the process data. The submodel parametric uncertainty has been propagated through the process model to allow for the estimation of the uncertainty in important process variables, notably $\mathrm{CO}_{2}$ capture percentage of the absorber and amount of $\mathrm{CO}_{2}$ regeneration in the stripper column. A sensitivity analysis has been performed to provide insight into the contribution of the uncertainty of certain parameters, and submodels, to the process uncertainty under various operating conditions.

As a stochastic model has been developed for the MEA-based $\mathrm{CO}_{2}$ capture system, it is recommended that it is used in future work for more rigorous performance studies. Most of the validation work presented here is focused on comparisons of the $\mathrm{CO}_{2}$ capture percentage in the absorber and $\mathrm{CO}_{2}$ loading in the stripper outlet, as well as column temperature profiles, to provide insight into the quality of the model fit to the data. It is recommended that future studies focus on quantifying the uncertainty in the process economics correlated with the submodel uncertainty. Moreover, optimization under uncertainty studies would be a valuable addition to the solvent modeling work. Although the model has been validated with NCCC data spread over a wide range of operating conditions, more work could be performed for validating the model at larger scales. The comparisons of TCM data to the model, however, have been useful in assessing the predictability of the model at varying scale. Further work must be performed to analyze the discrepancy in the model predictions of this plant's performance to the experimental data obtained. Further data to be obtained from this plant will potentially be incorporated into a more rigorous effort to validate the process model. 
In another ongoing project, a new campaign at NCCC has been planned using the MEA model developed in this work in an updated design of experiments methodology. The submodel parametric uncertainty has been propagated through the absorber model over a wide range of input variables, allowing for a rigorous study of how the process model uncertainty is dependent on conditions of operation. An initial set of test runs has been developed, with the objective of spreading out the runs over the input space of interest, while including a constraint that the $\mathrm{CO}_{2}$ capture percentage falls within a certain range in order to avoid having a large number of cases with operation at complete $\mathrm{CO}_{2}$ capture. The estimated confidence intervals in $\mathrm{CO}_{2}$ capture percentage, calculated from propagating parametric uncertainty through the process model, are also taken into consideration in the selection of the test runs, as it is desired to collect data in regions of relatively high uncertainty. A Bayesian framework has been developed for updating the parametric uncertainty as the data are collected, and updating the test plan in light of the change in the uncertainty estimate. Although the performance of the DOE methodology has been demonstrated in this work, it is recommended that future projects focus on a more efficient execution of this process. Due to implementation difficulties, multiple iterations of the algorithm, in which the distributions of the parameters are updated as new data are collected, and the change in the process uncertainty is used to update the test plan, were not obtained. An improvement in the algorithm for performing the Bayesian inference would be useful for improving the results of this project. This could be accomplished by improving the quality of the response surface model used as a surrogate for the absorber model, which could be difficult due to the complexity of the rate based column model. It will be useful to develop algorithms for generating accurate surrogate models by using lesser number of data points since evaluation of the rate-based model is computationally expensive. Alternatively, the actual model could be used for the Bayesian inference, so as to eliminate the effect of the surrogate on the accuracy of the posterior distributions obtained, although the computational expense of this method prevented its use in this work.

Another ongoing project for this work is based on developing a methodology for scale-up uncertainty quantification, in which a liquid distribution model for column packing will be implemented in the absorber model. This will allow for a study of the effect of the radial size of the column on its performance. Some preliminary work on this topic is presented in Appendix F of this document. Future work will focus on the implementation of this methodology into a 
process simulation, which will require a workaround to the general use of the plug flow assumption in simulators. Validation data for this model will be taken from the TCM pilot plant, considering that this is a relatively large scale plant for which the effect of liquid maldistribution is likely to play a role in column inefficiency. Moreover, data are available from this plant which contain information regarding the variation in the temperature profile over the column cross section, which could be implemented into the parameter calibration of the liquid distribution model.

Although the process model was developed using the industry standard aqueous MEA system as a baseline in this work, one of the major goals of this model was to develop it in a rigorous manner that can be applied to any new solvent model. Accordingly, another CCSI project related to this model involved a collaboration with an industry partner on the modeling of a high viscosity solvent system, although the detailed methodology and results of this project are not presented in this dissertation due to the proprietary nature of this solvent system. This project involved developing all submodels for the system from bench-scale data, using a similar methodology to that employed in this work for the MEA system, and incorporating them into an overall process model. This process has been developed for both bench-scale and pilot-scale (specifically NCCC) and validated with appropriate data, with promising results. 


\section{Appendix A: $\quad$ Physical Property Data (Standalone Models)}

\section{A.1 Viscosity Data}

As stated in Chapter 3.1, the data for viscosity $\left(\mu_{s l n}\right)$ are given in terms of temperature $(\mathrm{T}(\mathrm{K}))$, nominal MEA weight percentage $\left(W_{M E A}^{*}\right)$, and $\mathrm{CO}_{2}$ loading $(\alpha)$.

Table A.1 Viscosity data incorporated into model regression (Amundsen et al., 2009)

\begin{tabular}{|c|c|c|c|c|c|c|c|c|c|}
\hline \# & $W_{M E A}^{*}$ & $\mathrm{~T}(\mathrm{~K})$ & $\alpha$ & $\mu_{s l n}$ & $\#$ & $W_{M E A}^{*}$ & $\mathrm{~T}(\mathrm{~K})$ & $\alpha$ & $\mu_{s l n}$ \\
\hline 1 & 20 & 298.15 & 0 & 1.7 & 46 & 30 & 313.15 & 0.1 & 1.7 \\
\hline 2 & 30 & 298.15 & 0 & 2.48 & 47 & 30 & 313.15 & 0.2 & 2 \\
\hline 3 & 40 & 298.15 & 0 & 3.58 & 48 & 30 & 313.15 & 0.3 & 2 \\
\hline 4 & 20 & 313.15 & 0 & 1.18 & 49 & 30 & 313.15 & 0.4 & 2.4 \\
\hline 5 & 30 & 313.15 & 0 & 1.67 & 50 & 30 & 313.15 & 0.5 & 2.7 \\
\hline 6 & 40 & 313.15 & 0 & 2.28 & 51 & 30 & 323.15 & 0.1 & 1.4 \\
\hline 7 & 20 & 323.15 & 0 & 0.95 & 52 & 30 & 323.15 & 0.2 & 1.6 \\
\hline 8 & 30 & 323.15 & 0 & 1.33 & 53 & 30 & 323.15 & 0.3 & 1.6 \\
\hline 9 & 40 & 323.15 & 0 & 1.75 & 54 & 30 & 323.15 & 0.4 & 1.9 \\
\hline 10 & 20 & 343.15 & 0 & 0.67 & 55 & 30 & 323.15 & 0.5 & 2.1 \\
\hline 11 & 30 & 343.15 & 0 & 0.92 & 56 & 30 & 343.15 & 0.1 & 0.9 \\
\hline 12 & 40 & 343.15 & 0 & 1.14 & 57 & 30 & 343.15 & 0.2 & 1.1 \\
\hline 13 & 20 & 353.15 & 0 & 0.58 & 58 & 30 & 343.15 & 0.3 & 1.1 \\
\hline 14 & 30 & 353.15 & 0 & 0.77 & 59 & 30 & 343.15 & 0.4 & 1.3 \\
\hline 15 & 40 & 353.15 & 0 & 0.95 & 60 & 30 & 343.15 & 0.5 & 1.5 \\
\hline 16 & 20 & 298.15 & 0.1 & 1.8 & 61 & 30 & 353.15 & 0.1 & 0.8 \\
\hline 17 & 20 & 298.15 & 0.2 & 1.9 & 62 & 30 & 353.15 & 0.2 & 0.9 \\
\hline 18 & 20 & 298.15 & 0.3 & 1.9 & 63 & 30 & 353.15 & 0.3 & 0.9 \\
\hline 19 & 20 & 298.15 & 0.4 & 2.1 & 64 & 30 & 353.15 & 0.4 & 1.1 \\
\hline 20 & 20 & 298.15 & 0.5 & 2.2 & 65 & 30 & 353.15 & 0.5 & 1.3 \\
\hline 21 & 20 & 313.15 & 0.1 & 1.3 & 66 & 40 & 298.15 & 0.1 & 4 \\
\hline 22 & 20 & 313.15 & 0.2 & 1.3 & 67 & 40 & 298.15 & 0.2 & 4.6 \\
\hline 23 & 20 & 313.15 & 0.3 & 1.3 & 68 & 40 & 298.15 & 0.3 & 5.1 \\
\hline 24 & 20 & 313.15 & 0.4 & 1.4 & 69 & 40 & 298.15 & 0.4 & 6 \\
\hline 25 & 20 & 313.15 & 0.5 & 1.6 & 70 & 40 & 298.15 & 0.5 & 7 \\
\hline 26 & 20 & 323.15 & 0.1 & 1 & 71 & 40 & 313.15 & 0.1 & 2.5 \\
\hline 27 & 20 & 323.15 & 0.2 & 1 & 72 & 40 & 313.15 & 0.2 & 3 \\
\hline 28 & 20 & 323.15 & 0.3 & 1.1 & 73 & 40 & 313.15 & 0.3 & 3.3 \\
\hline 29 & 20 & 323.15 & 0.4 & 1.2 & 74 & 40 & 313.15 & 0.4 & 4 \\
\hline 30 & 20 & 323.15 & 0.5 & 1.3 & 75 & 40 & 313.15 & 0.5 & 4.6 \\
\hline 31 & 20 & 343.15 & 0.1 & 0.7 & 76 & 40 & 323.15 & 0.1 & 2 \\
\hline
\end{tabular}




\begin{tabular}{ccccc|ccccc}
32 & 20 & 343.15 & 0.2 & 0.7 & 77 & 40 & 323.15 & 0.2 & 2.3 \\
33 & 20 & 343.15 & 0.3 & 0.8 & 78 & 40 & 323.15 & 0.3 & 2.6 \\
34 & 20 & 343.15 & 0.4 & 0.8 & 79 & 40 & 323.15 & 0.4 & 3.1 \\
35 & 20 & 343.15 & 0.5 & 0.9 & 80 & 40 & 323.15 & 0.5 & 3.8 \\
36 & 20 & 353.15 & 0.1 & 0.6 & 81 & 40 & 343.15 & 0.1 & 1.3 \\
37 & 20 & 353.15 & 0.2 & 0.6 & 82 & 40 & 343.15 & 0.2 & 1.5 \\
38 & 20 & 353.15 & 0.3 & 0.7 & 83 & 40 & 343.15 & 0.3 & 1.7 \\
39 & 20 & 353.15 & 0.4 & 0.7 & 84 & 40 & 343.15 & 0.4 & 2 \\
40 & 20 & 353.15 & 0.5 & 0.8 & 85 & 40 & 343.15 & 0.5 & 2.3 \\
41 & 30 & 298.15 & 0.1 & 2.6 & 86 & 40 & 353.15 & 0.1 & 1.1 \\
42 & 30 & 298.15 & 0.2 & 2.9 & 87 & 40 & 353.15 & 0.2 & 1.3 \\
43 & 30 & 298.15 & 0.3 & 3.1 & 88 & 40 & 353.15 & 0.3 & 1.4 \\
44 & 30 & 298.15 & 0.4 & 3.5 & 89 & 40 & 353.15 & 0.4 & 1.7 \\
45 & 30 & 298.15 & 0.5 & 3.9 & 90 & 40 & 353.15 & 0.5 & 1.9 \\
\hline
\end{tabular}

\section{A.2 Density Data}

All density data are represented in units of $\mathrm{g} / \mathrm{cm}^{3}$. The data are given in Tables A.2-4.

Table A.2 Density data from Amundsen et al. (2012) incorporated into model.

\begin{tabular}{ccccc|ccccc}
\hline$\#$ & $\mathrm{~T}(\mathrm{~K})$ & $w_{M E A}^{*}$ & $\alpha$ & $\rho_{\text {sln }}$ & $\#$ & $\mathrm{~T}(\mathrm{~K})$ & $w_{M E A}^{*}$ & $\alpha$ & $\rho_{\text {sln }}$ \\
\hline 1 & 298.15 & 0.2 & 0 & 1.0053 & 43 & 323.15 & 0.3 & 0.1 & 1.016 \\
2 & 298.15 & 0.2 & 0.1 & 1.0188 & 44 & 323.15 & 0.3 & 0.2 & 1.0355 \\
3 & 298.15 & 0.2 & 0.2 & 1.0327 & 45 & 323.15 & 0.3 & 0.3 & 1.058 \\
4 & 298.15 & 0.2 & 0.3 & 1.0476 & 46 & 323.15 & 0.3 & 0.4 & 1.083 \\
5 & 298.15 & 0.2 & 0.4 & 1.064 & 47 & 323.15 & 0.3 & 0.5 & 1.108 \\
6 & 298.15 & 0.2 & 0.5 & 1.08 & 48 & 343.15 & 0.3 & 0 & 0.9858 \\
7 & 313.15 & 0.2 & 0 & 0.9991 & 49 & 343.15 & 0.3 & 0.1 & 1.004 \\
8 & 313.15 & 0.2 & 0.1 & 1.0125 & 50 & 343.15 & 0.3 & 0.2 & 1.024 \\
9 & 313.15 & 0.2 & 0.2 & 1.0264 & 51 & 343.15 & 0.3 & 0.3 & 1.0464 \\
10 & 313.15 & 0.2 & 0.3 & 1.0413 & 52 & 343.15 & 0.3 & 0.4 & 1.0719 \\
11 & 313.15 & 0.2 & 0.4 & 1.0579 & 53 & 353.15 & 0.3 & 0 & 0.9794 \\
12 & 313.15 & 0.2 & 0.5 & 1.0735 & 54 & 353.15 & 0.3 & 0.1 & 0.997 \\
13 & 323.15 & 0.2 & 0 & 0.9943 & 55 & 353.15 & 0.3 & 0.2 & 1.0176 \\
14 & 323.15 & 0.2 & 0.1 & 1.0076 & 56 & 353.15 & 0.3 & 0.3 & 1.0402 \\
15 & 323.15 & 0.2 & 0.2 & 1.0215 & 57 & 353.15 & 0.3 & 0.4 & 1.066 \\
16 & 323.15 & 0.2 & 0.3 & 1.0364 & 58 & 298.15 & 0.4 & 0 & 1.0158 \\
17 & 323.15 & 0.2 & 0.4 & 1.053 & 59 & 298.15 & 0.4 & 0.1 & 1.038 \\
18 & 323.15 & 0.2 & 0.5 & 1.068 & 60 & 298.15 & 0.4 & 0.2 & 1.063 \\
19 & 343.15 & 0.2 & 0 & 0.983 & 61 & 298.15 & 0.4 & 0.3 & 1.093 \\
20 & 343.15 & 0.2 & 0.1 & 0.9965 & 62 & 298.15 & 0.4 & 0.4 & 1.1285 \\
21 & 343.15 & 0.2 & 0.2 & 1.0105 & 63 & 298.15 & 0.4 & 0.5 & 1.1597 \\
22 & 343.15 & 0.2 & 0.3 & 1.0254 & 64 & 313.15 & 0.4 & 0 & 1.0077 \\
23 & 343.15 & 0.2 & 0.4 & 1.0419 & 65 & 313.15 & 0.4 & 0.1 & 1.03 \\
& & & & & & & & &
\end{tabular}




\begin{tabular}{ccccc|ccccc}
24 & 343.15 & 0.2 & 0.5 & 1.057 & 66 & 313.15 & 0.4 & 0.2 & 1.055 \\
25 & 353.15 & 0.2 & 0 & 0.9766 & 67 & 313.15 & 0.4 & 0.3 & 1.085 \\
26 & 353.15 & 0.2 & 0.1 & 0.9902 & 68 & 313.15 & 0.4 & 0.4 & 1.121 \\
27 & 353.15 & 0.2 & 0.2 & 1.0043 & 69 & 323.15 & 0.4 & 0 & 1.0018 \\
28 & 353.15 & 0.2 & 0.3 & 1.0192 & 70 & 323.15 & 0.4 & 0.1 & 1.024 \\
29 & 353.15 & 0.2 & 0.4 & 1.036 & 71 & 323.15 & 0.4 & 0.2 & 1.049 \\
30 & 298.15 & 0.3 & 0 & 1.0106 & 72 & 323.15 & 0.4 & 0.3 & 1.0797 \\
31 & 298.15 & 0.3 & 0.1 & 1.028 & 73 & 323.15 & 0.4 & 0.4 & 1.115 \\
32 & 298.15 & 0.3 & 0.2 & 1.048 & 74 & 343.15 & 0.4 & 0 & 0.9889 \\
33 & 298.15 & 0.3 & 0.3 & 1.07 & 75 & 343.15 & 0.4 & 0.1 & 1.012 \\
34 & 298.15 & 0.3 & 0.4 & 1.0957 & 76 & 343.15 & 0.4 & 0.2 & 1.037 \\
35 & 298.15 & 0.3 & 0.5 & 1.1211 & 77 & 343.15 & 0.4 & 0.3 & 1.068 \\
36 & 313.15 & 0.3 & 0 & 1.0034 & 78 & 343.15 & 0.4 & 0.4 & 1.104 \\
37 & 313.15 & 0.3 & 0.1 & 1.021 & 79 & 353.15 & 0.4 & 0 & 0.9819 \\
38 & 313.15 & 0.3 & 0.2 & 1.041 & 80 & 353.15 & 0.4 & 0.1 & 1.005 \\
39 & 313.15 & 0.3 & 0.3 & 1.0629 & 81 & 353.15 & 0.4 & 0.2 & 1.031 \\
40 & 313.15 & 0.3 & 0.4 & 1.0885 & 82 & 353.15 & 0.4 & 0.3 & 1.062 \\
41 & 313.15 & 0.3 & 0.5 & 1.114 & 83 & 353.15 & 0.4 & 0.4 & 1.0977 \\
42 & 323.15 & 0.3 & 0 & 0.9981 & & & & & \\
\hline
\end{tabular}

Table A.3 Density data from Jayarathna et al. (2013) incorporated into model.

\begin{tabular}{ccccc|ccccc}
\hline$\#$ & $\mathrm{~T}(\mathrm{~K})$ & $w_{M E A}^{*}$ & $\alpha$ & $\rho_{\operatorname{sln}}$ & $\#$ & $\mathrm{~T}(\mathrm{~K})$ & $w_{M E A}^{*}$ & $\alpha$ & $\rho_{\operatorname{sln}}$ \\
\hline 1 & 303.15 & 0.2 & 0 & 1.0036 & 37 & 323.15 & 0.3 & 0 & 0.9981 \\
2 & 303.15 & 0.2 & 0.1 & 1.0191 & 38 & 323.15 & 0.3 & 0.1 & 1.0178 \\
3 & 303.15 & 0.2 & 0.2 & 1.0324 & 39 & 323.15 & 0.3 & 0.2 & 1.0376 \\
4 & 303.15 & 0.2 & 0.3 & 1.0467 & 40 & 323.15 & 0.3 & 0.3 & 1.0563 \\
5 & 303.15 & 0.2 & 0.4 & 1.0611 & 41 & 323.15 & 0.3 & 0.4 & 1.0763 \\
6 & 303.15 & 0.2 & 0.5 & 1.0744 & 42 & 323.15 & 0.3 & 0.5 & 1.0958 \\
7 & 313.15 & 0.2 & 0 & 0.9994 & 43 & 333.15 & 0.3 & 0 & 0.9923 \\
8 & 313.15 & 0.2 & 0.1 & 1.0148 & 44 & 333.15 & 0.3 & 0.1 & 1.012 \\
9 & 313.15 & 0.2 & 0.2 & 1.0281 & 45 & 333.15 & 0.3 & 0.2 & 1.032 \\
10 & 313.15 & 0.2 & 0.3 & 1.0424 & 46 & 333.15 & 0.3 & 0.3 & 1.0507 \\
11 & 313.15 & 0.2 & 0.4 & 1.0567 & 47 & 333.15 & 0.3 & 0.4 & 1.0707 \\
12 & 313.15 & 0.2 & 0.5 & 1.0698 & 48 & 333.15 & 0.3 & 0.5 & 1.0901 \\
13 & 323.15 & 0.2 & 0 & 0.9946 & 49 & 303.15 & 0.4 & 0 & 1.0133 \\
14 & 323.15 & 0.2 & 0.1 & 1.0102 & 50 & 303.15 & 0.4 & 0.1 & 1.0378 \\
15 & 323.15 & 0.2 & 0.2 & 1.0233 & 51 & 303.15 & 0.4 & 0.2 & 1.0628 \\
16 & 323.15 & 0.2 & 0.3 & 1.0376 & 52 & 303.15 & 0.4 & 0.3 & 1.0876 \\
17 & 323.15 & 0.2 & 0.4 & 1.0518 & 53 & 303.15 & 0.4 & 0.4 & 1.1139 \\
18 & 323.15 & 0.2 & 0.5 & 1.0649 & 54 & 303.15 & 0.4 & 0.5 & 1.1396 \\
19 & 333.15 & 0.2 & 0 & 0.9891 & 55 & 313.15 & 0.4 & 0 & 1.0078 \\
20 & 333.15 & 0.2 & 0.1 & 1.0048 & 56 & 313.15 & 0.4 & 0.1 & 1.0325 \\
21 & 333.15 & 0.2 & 0.2 & 1.018 & 57 & 313.15 & 0.4 & 0.2 & 1.0576 \\
22 & 333.15 & 0.2 & 0.3 & 1.0323 & 58 & 313.15 & 0.4 & 0.3 & 1.0825
\end{tabular}




\begin{tabular}{ccccc|ccccc}
23 & 333.15 & 0.2 & 0.4 & 1.0464 & 59 & 313.15 & 0.4 & 0.4 & 1.1086 \\
24 & 333.15 & 0.2 & 0.5 & 1.0594 & 60 & 313.15 & 0.4 & 0.5 & 1.1344 \\
25 & 303.15 & 0.3 & 0 & 1.0082 & 61 & 323.15 & 0.4 & 0 & 1.0021 \\
26 & 303.15 & 0.3 & 0.1 & 1.0278 & 62 & 323.15 & 0.4 & 0.1 & 1.0269 \\
27 & 303.15 & 0.3 & 0.2 & 1.0475 & 63 & 323.15 & 0.4 & 0.2 & 1.0521 \\
28 & 303.15 & 0.3 & 0.3 & 1.0661 & 64 & 323.15 & 0.4 & 0.3 & 1.0771 \\
29 & 303.15 & 0.3 & 0.4 & 1.0863 & 65 & 323.15 & 0.4 & 0.4 & 1.1032 \\
30 & 303.15 & 0.3 & 0.5 & 1.106 & 66 & 323.15 & 0.4 & 0.5 & 1.129 \\
31 & 313.15 & 0.3 & 0 & 1.0033 & 67 & 333.15 & 0.4 & 0 & 0.9957 \\
32 & 313.15 & 0.3 & 0.1 & 1.0229 & 68 & 333.15 & 0.4 & 0.1 & 1.0208 \\
33 & 313.15 & 0.3 & 0.2 & 1.0427 & 69 & 333.15 & 0.4 & 0.2 & 1.0462 \\
34 & 313.15 & 0.3 & 0.3 & 1.0614 & 70 & 333.15 & 0.4 & 0.3 & 1.0713 \\
35 & 313.15 & 0.3 & 0.4 & 1.0815 & 71 & 333.15 & 0.4 & 0.4 & 1.0975 \\
36 & 313.15 & 0.3 & 0.5 & 1.101 & 72 & 333.15 & 0.4 & 0.5 & 1.1232 \\
\hline
\end{tabular}

Table A.4 Density data from Jayarathna et al. (2013) incorporated into model.

\begin{tabular}{ccccc|ccccc}
\hline$\#$ & $\mathrm{~T}(\mathrm{~K})$ & $w_{M E A}^{*}$ & $\alpha$ & $\rho_{\sin }$ & $\#$ & $\mathrm{~T}(\mathrm{~K})$ & $w_{M E A}^{*}$ & $\alpha$ & $\rho_{\operatorname{sln}}$ \\
\hline 1 & 298.15 & 0.3 & 0.1 & 1.0333 & 28 & 353.15 & 0.3 & 0.32 & 1.0434 \\
2 & 298.15 & 0.3 & 0.21 & 1.0534 & 29 & 353.15 & 0.3 & 0.44 & 1.066 \\
3 & 298.15 & 0.3 & 0.32 & 1.0756 & 30 & 353.15 & 0.3 & 0.56 & 1.0812 \\
4 & 298.15 & 0.3 & 0.44 & 1.0964 & 31 & 298.15 & 0.4 & 0.1 & 1.0376 \\
5 & 298.15 & 0.3 & 0.56 & 1.1142 & 32 & 298.15 & 0.4 & 0.21 & 1.0627 \\
6 & 313.15 & 0.3 & 0.1 & 1.0253 & 33 & 298.15 & 0.4 & 0.33 & 1.0945 \\
7 & 313.15 & 0.3 & 0.21 & 1.0464 & 34 & 298.15 & 0.4 & 0.45 & 1.1296 \\
8 & 313.15 & 0.3 & 0.32 & 1.0669 & 35 & 313.15 & 0.4 & 0.1 & 1.0295 \\
9 & 313.15 & 0.3 & 0.44 & 1.0891 & 36 & 313.15 & 0.4 & 0.21 & 1.0547 \\
10 & 313.15 & 0.3 & 0.56 & 1.1068 & 37 & 313.15 & 0.4 & 0.33 & 1.0867 \\
11 & 323.15 & 0.3 & 0.1 & 1.0196 & 38 & 313.15 & 0.4 & 0.45 & 1.1199 \\
12 & 323.15 & 0.3 & 0.21 & 1.0412 & 39 & 323.15 & 0.4 & 0.1 & 1.0237 \\
13 & 323.15 & 0.3 & 0.32 & 1.0613 & 40 & 323.15 & 0.4 & 0.21 & 1.049 \\
14 & 323.15 & 0.3 & 0.44 & 1.0838 & 41 & 323.15 & 0.4 & 0.33 & 1.0811 \\
15 & 323.15 & 0.3 & 0.56 & 1.1014 & 42 & 323.15 & 0.4 & 0.45 & 1.1138 \\
16 & 333.15 & 0.3 & 0.1 & 1.0138 & 43 & 333.15 & 0.4 & 0.1 & 1.0178 \\
17 & 333.15 & 0.3 & 0.21 & 1.0356 & 44 & 333.15 & 0.4 & 0.21 & 1.043 \\
18 & 333.15 & 0.3 & 0.32 & 1.0556 & 45 & 333.15 & 0.4 & 0.33 & 1.0752 \\
19 & 333.15 & 0.3 & 0.44 & 1.0782 & 46 & 333.15 & 0.4 & 0.45 & 1.1087 \\
20 & 333.15 & 0.3 & 0.56 & 1.0957 & 47 & 343.15 & 0.4 & 0.1 & 1.011 \\
21 & 343.15 & 0.3 & 0.1 & 1.0076 & 48 & 343.15 & 0.4 & 0.21 & 1.0367 \\
22 & 343.15 & 0.3 & 0.21 & 1.0297 & 49 & 343.15 & 0.4 & 0.33 & 1.0686 \\
23 & 343.15 & 0.3 & 0.32 & 1.0496 & 50 & 343.15 & 0.4 & 0.45 & 1.1032 \\
24 & 343.15 & 0.3 & 0.44 & 1.0723 & 51 & 353.15 & 0.4 & 0.1 & 1.0048 \\
25 & 343.15 & 0.3 & 0.56 & 1.0887 & 52 & 353.15 & 0.4 & 0.21 & 1.0292 \\
26 & 353.15 & 0.3 & 0.1 & 1.0002 & 53 & 353.15 & 0.4 & 0.33 & 1.0626 \\
27 & 353.15 & 0.3 & 0.21 & 1.0234 & 54 & 353.15 & 0.4 & 0.45 & 1.0963 \\
\hline
\end{tabular}




\section{A.3. Surface Tension Data}

The surface tension data are given in units of $\mathrm{N} / \mathrm{m}$.

Table A.5 Surface tension data incorporated into model (Jayarathna et al., 2013)

\begin{tabular}{|c|c|c|c|c|c|c|c|c|c|}
\hline$\#$ & $\mathrm{~T}(\mathrm{~K})$ & $w_{M E A}^{*}$ & $\alpha$ & $\sigma_{s l n}$ & \# & $\mathrm{T}(\mathrm{K})$ & $w_{M E A}^{*}$ & $\alpha$ & $\sigma_{s l n}$ \\
\hline 1 & 303.15 & 0.2 & 0 & 0.0667 & 37 & 323.15 & 0.3 & 0 & 0.061 \\
\hline 2 & 303.15 & 0.2 & 0.1 & 0.0676 & 38 & 323.15 & 0.3 & 0.1 & 0.062 \\
\hline 3 & 303.15 & 0.2 & 0.2 & 0.0684 & 39 & 323.15 & 0.3 & 0.2 & 0.0632 \\
\hline 4 & 303.15 & 0.2 & 0.3 & 0.0697 & 40 & 323.15 & 0.3 & 0.3 & 0.0647 \\
\hline 5 & 303.15 & 0.2 & 0.4 & 0.0714 & 41 & 323.15 & 0.3 & 0.4 & 0.0669 \\
\hline 6 & 303.15 & 0.2 & 0.5 & 0.0736 & 42 & 323.15 & 0.3 & 0.5 & 0.0698 \\
\hline 7 & 313.15 & 0.2 & 0 & 0.0652 & 43 & 333.15 & 0.3 & 0 & 0.0595 \\
\hline 8 & 313.15 & 0.2 & 0.1 & 0.0663 & 44 & 333.15 & 0.3 & 0.1 & 0.0605 \\
\hline 9 & 313.15 & 0.2 & 0.2 & 0.067 & 45 & 333.15 & 0.3 & 0.2 & 0.0615 \\
\hline 10 & 313.15 & 0.2 & 0.3 & 0.0679 & 46 & 333.15 & 0.3 & 0.3 & 0.0628 \\
\hline 11 & 313.15 & 0.2 & 0.4 & 0.07 & 47 & 333.15 & 0.3 & 0.4 & 0.0652 \\
\hline 12 & 313.15 & 0.2 & 0.5 & 0.0718 & 48 & 333.15 & 0.3 & 0.5 & 0.0685 \\
\hline 13 & 323.15 & 0.2 & 0 & 0.0636 & 49 & 303.15 & 0.4 & 0 & 0.0615 \\
\hline 14 & 323.15 & 0.2 & 0.1 & 0.0647 & 50 & 303.15 & 0.4 & 0.1 & 0.063 \\
\hline 15 & 323.15 & 0.2 & 0.2 & 0.0654 & 51 & 303.15 & 0.4 & 0.2 & 0.0646 \\
\hline 16 & 323.15 & 0.2 & 0.3 & 0.0664 & 52 & 303.15 & 0.4 & 0.3 & 0.0663 \\
\hline 17 & 323.15 & 0.2 & 0.4 & 0.0684 & 53 & 303.15 & 0.4 & 0.4 & 0.0693 \\
\hline 18 & 323.15 & 0.2 & 0.5 & 0.0702 & 54 & 303.15 & 0.4 & 0.5 & 0.0724 \\
\hline 19 & 333.15 & 0.2 & 0 & 0.0616 & 55 & 313.15 & 0.4 & 0 & 0.0598 \\
\hline 20 & 333.15 & 0.2 & 0.1 & 0.0631 & 56 & 313.15 & 0.4 & 0.1 & 0.0619 \\
\hline 21 & 333.15 & 0.2 & 0.2 & 0.0638 & 57 & 313.15 & 0.4 & 0.2 & 0.0634 \\
\hline 22 & 333.15 & 0.2 & 0.3 & 0.0649 & 58 & 313.15 & 0.4 & 0.3 & 0.0651 \\
\hline 23 & 333.15 & 0.2 & 0.4 & 0.0664 & 59 & 313.15 & 0.4 & 0.4 & 0.0681 \\
\hline 24 & 333.15 & 0.2 & 0.5 & 0.0689 & 60 & 313.15 & 0.4 & 0.5 & 0.0711 \\
\hline 25 & 303.15 & 0.3 & 0 & 0.0637 & 61 & 323.15 & 0.4 & 0 & 0.0584 \\
\hline 26 & 303.15 & 0.3 & 0.1 & 0.065 & 62 & 323.15 & 0.4 & 0.1 & 0.0603 \\
\hline 27 & 303.15 & 0.3 & 0.2 & 0.0664 & 63 & 323.15 & 0.4 & 0.2 & 0.062 \\
\hline 28 & 303.15 & 0.3 & 0.3 & 0.0678 & 64 & 323.15 & 0.4 & 0.3 & 0.0638 \\
\hline 29 & 303.15 & 0.3 & 0.4 & 0.0698 & 65 & 323.15 & 0.4 & 0.4 & 0.0667 \\
\hline 30 & 303.15 & 0.3 & 0.5 & 0.0728 & 66 & 323.15 & 0.4 & 0.5 & 0.0698 \\
\hline 31 & 313.15 & 0.3 & 0 & 0.0624 & 67 & 333.15 & 0.4 & 0 & 0.057 \\
\hline 32 & 313.15 & 0.3 & 0.1 & 0.0636 & 68 & 333.15 & 0.4 & 0.1 & 0.0587 \\
\hline 33 & 313.15 & 0.3 & 0.2 & 0.065 & 69 & 333.15 & 0.4 & 0.2 & 0.0602 \\
\hline 34 & 313.15 & 0.3 & 0.3 & 0.0662 & 70 & 333.15 & 0.4 & 0.3 & 0.0624 \\
\hline 35 & 313.15 & 0.3 & 0.4 & 0.0683 & 71 & 333.15 & 0.4 & 0.4 & 0.0649 \\
\hline 36 & 313.15 & 0.3 & 0.5 & 0.0714 & 72 & 333.15 & 0.4 & 0.5 & 0.0681 \\
\hline
\end{tabular}




\section{Appendix B: Physical Property Data (Thermodynamic Framework)}

\section{B.1 $\mathrm{CO}_{2}$ Partial Pressure}

The data are presented here in composition units of $\mathrm{CO}_{2}$ loading $(\alpha)$ and nominal MEA weight fraction $\left(w_{M E A}^{*}\right)$ for consistency, although the convention for reporting MEA concentration varies by source. Appropriate unit conversions have been applied here. All values of $P_{\mathrm{CO}_{2}}$ are given in units of $k P a$.

Table B.1 $\mathrm{CO}_{2}$ partial pressure data from Aronu et al. (2011)

\begin{tabular}{ccccc|ccccc}
\hline$\#$ & $\mathrm{~T}\left({ }^{\circ} \mathrm{C}\right)$ & $w_{M E A}^{*}$ & $\alpha$ & $P_{\mathrm{CO}_{2}}$ & $\#$ & $\mathrm{~T}\left({ }^{\circ} \mathrm{C}\right)$ & $w_{M E A}^{*}$ & $\alpha$ & $P_{\mathrm{CO}_{2}}$ \\
\hline 1 & 40 & 0.15 & 0.111 & 0.0017 & 41 & 40 & 0.3 & 0.421 & 0.3188 \\
2 & 40 & 0.15 & 0.148 & 0.0035 & 42 & 40 & 0.3 & 0.433 & 0.3809 \\
3 & 40 & 0.15 & 0.186 & 0.0068 & 43 & 40 & 0.3 & 0.447 & 0.5702 \\
4 & 40 & 0.15 & 0.22 & 0.017 & 44 & 40 & 0.3 & 0.464 & 1.0662 \\
5 & 40 & 0.15 & 0.236 & 0.0215 & 45 & 40 & 0.3 & 0.476 & 1.8326 \\
6 & 40 & 0.15 & 0.295 & 0.0427 & 46 & 40 & 0.3 & 0.477 & 1.8278 \\
7 & 40 & 0.15 & 0.298 & 0.045 & 47 & 40 & 0.3 & 0.485 & 2.3193 \\
8 & 40 & 0.15 & 0.342 & 0.0845 & 48 & 40 & 0.3 & 0.489 & 2.8577 \\
9 & 40 & 0.15 & 0.398 & 0.222 & 49 & 40 & 0.3 & 0.516 & 8.5583 \\
10 & 40 & 0.15 & 0.442 & 0.6634 & 50 & 40 & 0.3 & 0.524 & 11.812 \\
11 & 40 & 0.15 & 0.45 & 0.7013 & 51 & 60 & 0.3 & 0.053 & 0.0045 \\
12 & 40 & 0.15 & 0.516 & 4.8405 & 52 & 60 & 0.3 & 0.105 & 0.0154 \\
13 & 40 & 0.15 & 0.529 & 7.8861 & 53 & 60 & 0.3 & 0.162 & 0.0427 \\
14 & 40 & 0.15 & 0.565 & 16.0024 & 54 & 60 & 0.3 & 0.244 & 0.1348 \\
15 & 60 & 0.15 & 0.048 & 0.0042 & 55 & 60 & 0.3 & 0.303 & 0.3015 \\
16 & 60 & 0.15 & 0.06 & 0.0056 & 56 & 60 & 0.3 & 0.36 & 0.6436 \\
17 & 60 & 0.15 & 0.075 & 0.0068 & 57 & 60 & 0.3 & 0.393 & 1.097 \\
18 & 60 & 0.15 & 0.069 & 0.0078 & 58 & 60 & 0.3 & 0.428 & 2.5014 \\
19 & 60 & 0.15 & 0.098 & 0.0094 & 59 & 60 & 0.3 & 0.491 & 13.558 \\
20 & 60 & 0.15 & 0.135 & 0.0151 & 60 & 80 & 0.3 & 0.017 & 0.0056 \\
21 & 60 & 0.15 & 0.144 & 0.0234 & 61 & 80 & 0.3 & 0.04 & 0.0219 \\
22 & 60 & 0.15 & 0.175 & 0.0417 & 62 & 80 & 0.3 & 0.075 & 0.0557 \\
23 & 60 & 0.15 & 0.23 & 0.0965 & 63 & 80 & 0.3 & 0.122 & 0.1406 \\
24 & 60 & 0.15 & 0.253 & 0.1462 & 64 & 80 & 0.3 & 0.155 & 0.2485 \\
25 & 60 & 0.15 & 0.415 & 1.7998 & 65 & 80 & 0.3 & 0.216 & 0.6137 \\
26 & 60 & 0.15 & 0.48 & 5.409 & 66 & 80 & 0.3 & 0.271 & 1.2538 \\
27 & 60 & 0.15 & 0.492 & 8.2297 & 67 & 80 & 0.3 & 0.347 & 3.7522 \\
28 & 80 & 0.15 & 0.103 & 0.0603 & 68 & 80 & 0.3 & 0.4 & 7.9387 \\
29 & 80 & 0.15 & 0.147 & 0.1315 & 69 & 80 & 0.3 & 0.398 & 8.3031 \\
30 & 80 & 0.15 & 0.211 & 0.3544 & 70 & 40 & 0.45 & 0.141 & 0.0035
\end{tabular}




\begin{tabular}{ccccc|ccccc}
31 & 80 & 0.15 & 0.242 & 0.525 & 71 & 40 & 0.45 & 0.148 & 0.0035 \\
32 & 80 & 0.15 & 0.373 & 3.6374 & 72 & 40 & 0.45 & 0.195 & 0.0077 \\
33 & 80 & 0.15 & 0.409 & 6.3092 & 73 & 40 & 0.45 & 0.217 & 0.0099 \\
34 & 40 & 0.3 & 0.102 & 0.0016 & 74 & 40 & 0.45 & 0.234 & 0.0123 \\
35 & 40 & 0.3 & 0.206 & 0.0123 & 75 & 40 & 0.45 & 0.276 & 0.0164 \\
36 & 40 & 0.3 & 0.25 & 0.0246 & 76 & 40 & 0.45 & 0.271 & 0.0178 \\
37 & 40 & 0.3 & 0.337 & 0.0603 & 77 & 40 & 0.45 & 0.3 & 0.0364 \\
38 & 40 & 0.3 & 0.353 & 0.0851 & 78 & 40 & 0.45 & 0.354 & 0.0598 \\
39 & 40 & 0.3 & 0.401 & 0.1835 & 79 & 40 & 0.45 & 0.39 & 0.1087 \\
40 & 40 & 0.3 & 0.417 & 0.2928 & 80 & 40 & 0.45 & 0.404 & 0.1781 \\
\hline
\end{tabular}

Table B.1 $\mathrm{CO}_{2}$ partial pressure data from Aronu et al. (2011) (cont.)

\begin{tabular}{|c|c|c|c|c|c|c|c|c|c|}
\hline$\#$ & $\mathrm{~T}\left({ }^{\circ} \mathrm{C}\right)$ & $w_{M E A}^{*}$ & $\alpha$ & $P_{\mathrm{CO}_{2}}$ & $\#$ & $\mathrm{~T}\left({ }^{\circ} \mathrm{C}\right)$ & $w_{M E A}^{*}$ & $\alpha$ & $P_{\mathrm{CO}_{2}}$ \\
\hline 81 & 40 & 0.45 & 0.428 & 0.2787 & 110 & 40 & 0.6 & 0.344 & 0.0526 \\
\hline 82 & 40 & 0.45 & 0.464 & 0.9173 & 111 & 40 & 0.6 & 0.394 & 0.1508 \\
\hline 83 & 40 & 0.45 & 0.475 & 2.1609 & 112 & 40 & 0.6 & 0.427 & 0.3824 \\
\hline 84 & 40 & 0.45 & 0.497 & 5.4871 & 113 & 40 & 0.6 & 0.449 & 0.9062 \\
\hline 85 & 60 & 0.45 & 0.045 & 0.0019 & 114 & 40 & 0.6 & 0.468 & 1.5153 \\
\hline 86 & 60 & 0.45 & 0.087 & 0.0059 & 115 & 40 & 0.6 & 0.481 & 3.7472 \\
\hline 87 & 60 & 0.45 & 0.12 & 0.0099 & 116 & 40 & 0.6 & 0.5 & 12.472 \\
\hline 88 & 60 & 0.45 & 0.169 & 0.0205 & 117 & 60 & 0.6 & 0.046 & 0.0007 \\
\hline 89 & 60 & 0.45 & 0.232 & 0.0787 & 118 & 60 & 0.6 & 0.126 & 0.011 \\
\hline 90 & 60 & 0.45 & 0.269 & 0.1284 & 119 & 60 & 0.6 & 0.172 & 0.0341 \\
\hline 91 & 60 & 0.45 & 0.352 & 0.4279 & 120 & 60 & 0.6 & 0.248 & 0.1097 \\
\hline 92 & 60 & 0.45 & 0.392 & 1.4259 & 121 & 60 & 0.6 & 0.316 & 0.2933 \\
\hline 93 & 60 & 0.45 & 0.454 & 4.6349 & 122 & 60 & 0.6 & 0.382 & 0.8475 \\
\hline 94 & 60 & 0.45 & 0.46 & 6.2928 & 123 & 60 & 0.6 & 0.424 & 3.0267 \\
\hline 95 & 60 & 0.45 & 0.471 & 8.29 & 124 & 60 & 0.6 & 0.457 & 8.2258 \\
\hline 96 & 80 & 0.45 & 0.017 & 0.0008 & 125 & 60 & 0.6 & 0.48 & 18.967 \\
\hline 97 & 80 & 0.45 & 0.027 & 0.0023 & 126 & 80 & 0.6 & 0.018 & 0.002 \\
\hline 98 & 80 & 0.45 & 0.038 & 0.0056 & 127 & 80 & 0.6 & 0.056 & 0.017 \\
\hline 99 & 80 & 0.45 & 0.025 & 0.006 & 128 & 80 & 0.6 & 0.073 & 0.0325 \\
\hline 100 & 80 & 0.45 & 0.061 & 0.0099 & 129 & 80 & 0.6 & 0.124 & 0.0777 \\
\hline 101 & 80 & 0.45 & 0.086 & 0.0288 & 130 & 80 & 0.6 & 0.162 & 0.161 \\
\hline 102 & 80 & 0.45 & 0.109 & 0.0529 & 131 & 80 & 0.6 & 0.191 & 0.2513 \\
\hline 103 & 80 & 0.45 & 0.135 & 0.1236 & 132 & 80 & 0.6 & 0.238 & 0.5431 \\
\hline 104 & 80 & 0.45 & 0.236 & 0.3981 & 133 & 80 & 0.6 & 0.264 & 0.8699 \\
\hline 105 & 80 & 0.45 & 0.389 & 4.5002 & 134 & 80 & 0.6 & 0.308 & 1.6522 \\
\hline 106 & 80 & 0.45 & 0.435 & 11.249 & 135 & 80 & 0.6 & 0.352 & 3.43 \\
\hline 107 & 40 & 0.6 & 0.173 & 0.006 & 136 & 80 & 0.6 & 0.387 & 6.0947 \\
\hline 108 & 40 & 0.6 & 0.242 & 0.0127 & 137 & 80 & 0.6 & 0.404 & 9.0463 \\
\hline 109 & 40 & 0.6 & 0.306 & 0.0281 & 138 & 80 & 0.6 & 0.416 & 11.271 \\
\hline
\end{tabular}


Table B.2 $\mathrm{CO}_{2}$ partial pressure data from Hilliard (2008)

\begin{tabular}{ccccc|ccccc}
\hline$\#$ & $\mathrm{~T}\left({ }^{\circ} \mathrm{C}\right)$ & $w_{M E A}^{*}$ & $\alpha$ & $P_{\mathrm{CO}_{2}}$ & $\#$ & $\mathrm{~T}\left({ }^{\circ} \mathrm{C}\right)$ & $w_{M E A}^{*}$ & $\alpha$ & $P_{\mathrm{CO}_{2}}$ \\
\hline 1 & 39.98 & 0.177 & 0.121 & 0.00555 & 29 & 40.00 & 0.301 & 0.4 & 0.128 \\
2 & 40.02 & 0.174 & 0.212 & 0.014 & 30 & 40.02 & 0.305 & 0.464 & 0.75 \\
3 & 39.94 & 0.177 & 0.3 & 0.0362 & 31 & 39.93 & 0.300 & 0.466 & 0.574 \\
4 & 40.08 & 0.178 & 0.369 & 0.116 & 32 & 39.99 & 0.301 & 0.481 & 0.883 \\
5 & 40.00 & 0.179 & 0.467 & 0.879 & 33 & 39.88 & 0.303 & 0.491 & 1.1 \\
6 & 39.70 & 0.176 & 0.552 & 8.56 & 34 & 40.02 & 0.301 & 0.501 & 1.87 \\
7 & 59.95 & 0.179 & 0.159 & 0.0212 & 35 & 40.00 & 0.301 & 0.518 & 3.03 \\
8 & 60.06 & 0.181 & 0.219 & 0.078 & 36 & 40.00 & 0.303 & 0.591 & 28.3 \\
9 & 60.04 & 0.177 & 0.307 & 0.244 & 37 & 59.87 & 0.300 & 0.114 & 0.0194 \\
10 & 60.02 & 0.179 & 0.38 & 0.794 & 38 & 59.96 & 0.302 & 0.191 & 0.0589 \\
11 & 59.94 & 0.178 & 0.477 & 4.32 & 39 & 59.96 & 0.302 & 0.291 & 0.209 \\
12 & 60.01 & 0.178 & 0.504 & 14.8 & 40 & 59.88 & 0.300 & 0.386 & 0.763 \\
13 & 39.99 & 0.296 & 0.153 & 0.0057 & 41 & 59.77 & 0.304 & 0.485 & 4.86 \\
14 & 40.06 & 0.298 & 0.163 & 0.00664 & 42 & 60.11 & 0.305 & 0.544 & 25.8 \\
15 & 39.99 & 0.299 & 0.17 & 0.00721 & 43 & 59.95 & 0.311 & 0.565 & 50.2 \\
16 & 40.14 & 0.299 & 0.191 & 0.00995 & 44 & 39.99 & 0.402 & 0.115 & 0.00505 \\
17 & 40.03 & 0.295 & 0.194 & 0.00985 & 45 & 40.02 & 0.396 & 0.201 & 0.0108 \\
18 & 40.03 & 0.301 & 0.232 & 0.0146 & 46 & 39.94 & 0.400 & 0.298 & 0.0295 \\
19 & 40.12 & 0.302 & 0.246 & 0.0191 & 47 & 40.11 & 0.408 & 0.373 & 0.104 \\
20 & 39.97 & 0.303 & 0.269 & 0.0231 & 48 & 40.00 & 0.403 & 0.485 & 1.62 \\
21 & 40.35 & 0.297 & 0.272 & 0.0224 & 49 & 39.97 & 0.404 & 0.545 & 22.3 \\
22 & 39.87 & 0.301 & 0.326 & 0.0485 & 50 & 60.00 & 0.406 & 0.136 & 0.0155 \\
23 & 39.88 & 0.301 & 0.348 & 0.0662 & 51 & 60.04 & 0.406 & 0.225 & 0.0731 \\
24 & 39.99 & 0.301 & 0.35 & 0.0721 & 52 & 59.99 & 0.404 & 0.291 & 0.199 \\
25 & 39.87 & 0.303 & 0.36 & 0.0966 & 53 & 60.04 & 0.410 & 0.415 & 0.847 \\
26 & 40.05 & 0.316 & 0.382 & 0.131 & 54 & 59.93 & 0.409 & 0.464 & 6.98 \\
27 & 39.88 & 0.301 & 0.386 & 0.12 & 55 & 60.00 & 0.401 & 0.502 & 26.5 \\
28 & 39.85 & 0.301 & 0.389 & 0.113 & & & & & \\
\hline & & & & & & & & &
\end{tabular}

Table $\mathrm{B.3} \mathrm{CO}_{2}$ partial pressure data from Jou et al. (1995)

\begin{tabular}{ccccc|ccccc}
\hline$\#$ & $\mathrm{~T}\left({ }^{\circ} \mathrm{C}\right)$ & $w_{M E A}^{*}$ & $\alpha$ & $P_{\mathrm{CO}_{2}}$ & $\#$ & $\mathrm{~T}\left({ }^{\circ} \mathrm{C}\right)$ & $w_{M E A}^{*}$ & $\alpha$ & $P_{\mathrm{CO}_{2}}$ \\
\hline 1 & 25 & 0.3 & 0.211 & 0.00213 & 25 & 80 & 0.3 & 0.187 & 0.278 \\
2 & 25 & 0.3 & 0.323 & 0.00927 & 26 & 80 & 0.3 & 0.348 & 2.67 \\
3 & 25 & 0.3 & 0.439 & 0.06 & 27 & 80 & 0.3 & 0.46 & 16 \\
4 & 25 & 0.3 & 0.494 & 0.392 & 28 & 80 & 0.3 & 0.517 & 56
\end{tabular}




\begin{tabular}{ccccc|ccccc}
5 & 25 & 0.3 & 0.54 & 2.8 & 29 & 80 & 0.3 & 0.576 & 235 \\
6 & 25 & 0.3 & 0.583 & 11.8 & 30 & 100 & 0.3 & 0.0117 & 0.00724 \\
7 & 40 & 0.3 & 0.0888 & 0.00147 & 31 & 100 & 0.3 & 0.0566 & 0.136 \\
8 & 40 & 0.3 & 0.203 & 0.00896 & 32 & 100 & 0.3 & 0.188 & 1.43 \\
9 & 40 & 0.3 & 0.365 & 0.0677 & 33 & 100 & 0.3 & 0.381 & 19 \\
10 & 40 & 0.3 & 0.461 & 0.604 & 34 & 100 & 0.3 & 0.422 & 39 \\
11 & 40 & 0.3 & 0.513 & 2.57 & 35 & 100 & 0.3 & 0.477 & 69 \\
12 & 40 & 0.3 & 0.557 & 8.09 & 36 & 100 & 0.3 & 0.481 & 109 \\
13 & 60 & 0.3 & 0.0564 & 0.00428 & 37 & 100 & 0.3 & 0.571 & 509 \\
14 & 60 & 0.3 & 0.119 & 0.0193 & 38 & 100 & 0.3 & 0.589 & 376 \\
15 & 60 & 0.3 & 0.206 & 0.0579 & 39 & 120 & 0.3 & 0.00333 & 0.00202 \\
16 & 60 & 0.3 & 0.389 & 0.528 & 40 & 120 & 0.3 & 0.0112 & 0.0221 \\
17 & 60 & 0.3 & 0.438 & 2.01 & 41 & 120 & 0.3 & 0.0247 & 0.0984 \\
18 & 60 & 0.3 & 0.504 & 11 & 42 & 120 & 0.3 & 0.119 & 2.29 \\
19 & 60 & 0.3 & 0.565 & 34.1 & 43 & 120 & 0.3 & 0.349 & 46.8 \\
20 & 60 & 0.3 & 0.575 & 82 & 44 & 120 & 0.3 & 0.403 & 122 \\
21 & 80 & 0.3 & 0.0174 & 0.00296 & 45 & 120 & 0.3 & 0.444 & 222 \\
22 & 80 & 0.3 & 0.0236 & 0.00484 & 46 & 120 & 0.3 & 0.473 & 422 \\
23 & 80 & 0.3 & 0.0781 & 0.0465 & 47 & 120 & 0.3 & 0.536 & 822 \\
24 & 80 & 0.3 & 0.118 & 0.0992 & & & & & \\
\hline
\end{tabular}

Table $\mathrm{B} .4 \mathrm{CO}_{2}$ partial pressure data from Dugas (2009)

\begin{tabular}{ccccc|ccccc}
\hline$\#$ & $\mathrm{~T}\left({ }^{\circ} \mathrm{C}\right)$ & $w_{M E A}^{*}$ & $\alpha$ & $P_{\mathrm{CO}_{2}}$ & $\#$ & $\mathrm{~T}\left({ }^{\circ} \mathrm{C}\right)$ & $w_{M E A}^{*}$ & $\alpha$ & $P_{\mathrm{CO}_{2}}$ \\
\hline 1 & 40 & 0.29951 & 0.252 & 0.0157 & 26 & 100 & 0.35473 & 0.356 & 21.534 \\
2 & 40 & 0.29951 & 0.351 & 0.077 & 27 & 40 & 0.40188 & 0.261 & 0.014 \\
3 & 40 & 0.29951 & 0.432 & 0.465 & 28 & 40 & 0.40188 & 0.353 & 0.067 \\
4 & 40 & 0.29951 & 0.496 & 4.216 & 29 & 40 & 0.40188 & 0.428 & 0.434 \\
5 & 60 & 0.29951 & 0.252 & 0.109 & 30 & 40 & 0.40188 & 0.461 & 1.509 \\
6 & 60 & 0.29951 & 0.351 & 0.66 & 31 & 60 & 0.40188 & 0.261 & 0.096 \\
7 & 60 & 0.29951 & 0.432 & 3.434 & 32 & 60 & 0.40188 & 0.353 & 0.634 \\
8 & 60 & 0.29951 & 0.496 & 16.157 & 33 & 60 & 0.40188 & 0.428 & 3.463 \\
9 & 80 & 0.29951 & 0.271 & 1.053 & 34 & 60 & 0.40188 & 0.461 & 8.171 \\
10 & 80 & 0.29951 & 0.366 & 4.443 & 35 & 80 & 0.40188 & 0.256 & 0.86 \\
11 & 100 & 0.29951 & 0.271 & 5.297 & 36 & 80 & 0.40188 & 0.359 & 3.923 \\
12 & 100 & 0.29951 & 0.366 & 19.008 & 37 & 100 & 0.40188 & 0.256 & 4.274 \\
13 & 40 & 0.35473 & 0.231 & 0.0104 & 38 & 100 & 0.40188 & 0.359 & 18.657 \\
14 & 40 & 0.35473 & 0.324 & 0.034 & 39 & 40 & 0.44261 & 0.252 & 0.0123 \\
15 & 40 & 0.35473 & 0.382 & 0.107 & 40 & 40 & 0.44261 & 0.372 & 0.084 \\
16 & 40 & 0.35473 & 0.441 & 0.417 & 41 & 40 & 0.44261 & 0.435 & 0.491 \\
17 & 40 & 0.35473 & 0.496 & 5.354 & 42 & 40 & 0.44261 & 0.502 & 8.792 \\
18 & 60 & 0.35473 & 0.231 & 0.061 & 43 & 60 & 0.44261 & 0.252 & 0.1 \\
19 & 60 & 0.35473 & 0.324 & 0.263 & 44 & 60 & 0.44261 & 0.372 & 0.694 \\
20 & 60 & 0.35473 & 0.382 & 0.892 & 45 & 60 & 0.44261 & 0.435 & 3.859 \\
21 & 60 & 0.35473 & 0.441 & 2.862 & 46 & 60 & 0.44261 & 0.502 & 29.427
\end{tabular}




\begin{tabular}{ccccc|ccccc}
22 & 60 & 0.35473 & 0.496 & 21.249 & 47 & 80 & 0.44261 & 0.254 & 0.873 \\
23 & 80 & 0.35473 & 0.265 & 0.979 & 48 & 80 & 0.44261 & 0.355 & 3.964 \\
24 & 80 & 0.35473 & 0.356 & 4.797 & 49 & 100 & 0.44261 & 0.254 & 3.876 \\
25 & 100 & 0.35473 & 0.265 & 4.94 & 50 & 100 & 0.44261 & 0.355 & 18.406 \\
\hline
\end{tabular}

Table $\mathrm{B.5} \mathrm{CO}_{2}$ partial pressure data from Lee et al. (1976)

\begin{tabular}{|c|c|c|c|c|c|c|c|c|c|}
\hline \# & $\mathrm{T}\left({ }^{\circ} \mathrm{C}\right)$ & $w_{M E A}^{*}$ & $\alpha$ & $P_{\mathrm{CO}_{2}}$ & $\#$ & $\mathrm{~T}\left({ }^{\circ} \mathrm{C}\right)$ & $w_{M E A}^{*}$ & $\alpha$ & $P_{\mathrm{CO}_{2}}$ \\
\hline 1 & 25 & 0.0613 & 0.417 & 0.1 & 41 & 60 & 0.1554 & 0.305 & 0.316 \\
\hline 2 & 40 & 0.0616 & 0.328 & 0.1 & 42 & 80 & 0.1574 & 0.214 & 0.316 \\
\hline 3 & 60 & 0.0622 & 0.244 & 0.1 & 43 & 100 & 0.1598 & 0.116 & 0.316 \\
\hline 4 & 80 & 0.0629 & 0.156 & 0.1 & 44 & 25 & 0.1529 & 0.5 & 1 \\
\hline 5 & 100 & 0.0639 & 0.077 & 0.1 & 45 & 40 & 0.1538 & 0.437 & 1 \\
\hline 6 & 25 & 0.0613 & 0.46 & 0.316 & 46 & 60 & 0.1554 & 0.368 & 1 \\
\hline 7 & 40 & 0.0616 & 0.39 & 0.316 & 47 & 80 & 0.1574 & 0.274 & 1 \\
\hline 8 & 60 & 0.0622 & 0.307 & 0.316 & 48 & 100 & 0.1598 & 0.168 & 1 \\
\hline 9 & 80 & 0.0629 & 0.216 & 0.316 & 49 & 120 & 0.1627 & 0.065 & 1 \\
\hline 10 & 100 & 0.0639 & 0.119 & 0.316 & 50 & 25 & 0.1529 & 0.543 & 3.16 \\
\hline 11 & 25 & 0.0613 & 0.513 & 1 & 51 & 40 & 0.1538 & 0.488 & 3.16 \\
\hline 12 & 40 & 0.0616 & 0.451 & 1 & 52 & 60 & 0.1554 & 0.423 & 3.16 \\
\hline 13 & 60 & 0.0622 & 0.372 & 1 & 53 & 80 & 0.1574 & 0.336 & 3.16 \\
\hline 14 & 80 & 0.0629 & 0.275 & 1 & 54 & 100 & 0.1598 & 0.23 & 3.16 \\
\hline 15 & 100 & 0.0639 & 0.172 & 1 & 55 & 120 & 0.1627 & 0.13 & 3.16 \\
\hline 16 & 120 & 0.065 & 0.077 & 1 & 56 & 25 & 0.1529 & 0.593 & 10 \\
\hline 17 & 25 & 0.0613 & 0.581 & 3.16 & 57 & 40 & 0.1538 & 0.538 & 10 \\
\hline 18 & 40 & 0.0616 & 0.521 & 3.16 & 58 & 60 & 0.1554 & 0.482 & 10 \\
\hline 19 & 60 & 0.0622 & 0.442 & 3.16 & 59 & 80 & 0.1574 & 0.403 & 10 \\
\hline 20 & 80 & 0.0629 & 0.343 & 3.16 & 60 & 100 & 0.1598 & 0.302 & 10 \\
\hline 21 & 100 & 0.0639 & 0.237 & 3.16 & 61 & 120 & 0.1627 & 0.211 & 10 \\
\hline 22 & 120 & 0.065 & 0.14 & 3.16 & 62 & 40 & 0.1538 & 0.595 & 31.6 \\
\hline 23 & 40 & 0.0616 & 0.6 & 10 & 63 & 60 & 0.1554 & 0.541 & 31.6 \\
\hline 24 & 60 & 0.0622 & 0.52 & 10 & 64 & 80 & 0.1574 & 0.478 & 31.6 \\
\hline 25 & 80 & 0.0629 & 0.427 & 10 & 65 & 100 & 0.1598 & 0.385 & 31.6 \\
\hline 26 & 100 & 0.0639 & 0.314 & 10 & 66 & 120 & 0.1627 & 0.293 & 31.6 \\
\hline 27 & 120 & 0.065 & 0.231 & 10 & 67 & 80 & 0.1574 & 0.552 & 100 \\
\hline 28 & 80 & 0.0629 & 0.523 & 31.6 & 68 & 100 & 0.1598 & 0.475 & 100 \\
\hline 29 & 100 & 0.0639 & 0.405 & 31.6 & 69 & 120 & 0.1627 & 0.39 & 100 \\
\hline 30 & 120 & 0.065 & 0.328 & 31.6 & 70 & 100 & 0.1598 & 0.57 & 316 \\
\hline 31 & 100 & 0.0639 & 0.513 & 100 & 71 & 120 & 0.1627 & 0.498 & 316 \\
\hline 32 & 120 & 0.065 & 0.436 & 100 & 72 & 25 & 0.2291 & 0.414 & 0.1 \\
\hline 33 & 120 & 0.065 & 0.565 & 316 & 73 & 40 & 0.2306 & 0.326 & 0.1 \\
\hline 34 & 25 & 0.1529 & 0.415 & 0.1 & 74 & 60 & 0.2331 & 0.24 & 0.1 \\
\hline 35 & 40 & 0.1538 & 0.327 & 0.1 & 75 & 80 & 0.2361 & 0.153 & 0.1 \\
\hline 36 & 60 & 0.1554 & 0.241 & 0.1 & 76 & 100 & 0.2398 & 0.072 & 0.1 \\
\hline 37 & 80 & 0.1574 & 0.154 & 0.1 & 77 & 25 & 0.2291 & 0.453 & 0.316 \\
\hline
\end{tabular}




\begin{tabular}{lcccc|ccccc}
38 & 100 & 0.1598 & 0.074 & 0.1 & 78 & 40 & 0.2306 & 0.38 & 0.316 \\
39 & 25 & 0.1529 & 0.457 & 0.316 & 79 & 60 & 0.2331 & 0.304 & 0.316 \\
40 & 40 & 0.1538 & 0.383 & 0.316 & 80 & 80 & 0.2361 & 0.213 & 0.316 \\
\hline
\end{tabular}

Table $\mathrm{B.5}^{\mathrm{CO}_{2}}$ partial pressure data from Lee et al. (1976) (cont.)

\begin{tabular}{|c|c|c|c|c|c|c|c|c|c|}
\hline$\#$ & $\mathrm{~T}\left({ }^{\circ} \mathrm{C}\right)$ & $w_{M E A}^{*}$ & $\alpha$ & $P_{\mathrm{CO}_{2}}$ & \# & $\mathrm{T}\left({ }^{\circ} \mathrm{C}\right)$ & $w_{M E A}^{*}$ & $\alpha$ & $P_{\mathrm{CO}_{2}}$ \\
\hline 81 & 100 & 0.2398 & 0.114 & 0.316 & 119 & 40 & 0.3072 & 0.379 & 0.316 \\
\hline 82 & 25 & 0.2291 & 0.49 & 1 & 120 & 60 & 0.3107 & 0.303 & 0.316 \\
\hline 83 & 40 & 0.2306 & 0.428 & 1 & 121 & 80 & 0.3149 & 0.212 & 0.316 \\
\hline 84 & 60 & 0.2331 & 0.365 & 1 & 122 & 100 & 0.3199 & 0.112 & 0.316 \\
\hline 85 & 80 & 0.2361 & 0.273 & 1 & 123 & 25 & 0.3051 & 0.483 & 1 \\
\hline 86 & 100 & 0.2398 & 0.164 & 1 & 124 & 40 & 0.3072 & 0.427 & 1 \\
\hline 87 & 120 & 0.2441 & 0.065 & 1 & 125 & 60 & 0.3107 & 0.363 & 1 \\
\hline 88 & 25 & 0.2291 & 0.521 & 3.16 & 126 & 80 & 0.3149 & 0.272 & 1 \\
\hline 89 & 40 & 0.2306 & 0.469 & 3.16 & 127 & 100 & 0.3199 & 0.161 & 1 \\
\hline 90 & 60 & 0.2331 & 0.417 & 3.16 & 128 & 120 & 0.3256 & 0.065 & 1 \\
\hline 91 & 80 & 0.2361 & 0.334 & 3.16 & 129 & 25 & 0.3051 & 0.513 & 3.16 \\
\hline 92 & 100 & 0.2398 & 0.225 & 3.16 & 130 & 40 & 0.3072 & 0.468 & 3.16 \\
\hline 93 & 120 & 0.2441 & 0.13 & 3.16 & 131 & 60 & 0.3107 & 0.416 & 3.16 \\
\hline 94 & 25 & 0.2291 & 0.557 & 10 & 132 & 80 & 0.3149 & 0.332 & 3.16 \\
\hline 95 & 40 & 0.2306 & 0.51 & 10 & 133 & 100 & 0.3199 & 0.221 & 3.16 \\
\hline 96 & 60 & 0.2331 & 0.463 & 10 & 134 & 120 & 0.3256 & 0.13 & 3.16 \\
\hline 97 & 80 & 0.2361 & 0.395 & 10 & 135 & 25 & 0.3051 & 0.553 & 10 \\
\hline 98 & 100 & 0.2398 & 0.294 & 10 & 136 & 40 & 0.3072 & 0.508 & 10 \\
\hline 99 & 120 & 0.2441 & 0.205 & 10 & 137 & 60 & 0.3107 & 0.46 & 10 \\
\hline 100 & 40 & 0.2306 & 0.563 & 31.6 & 138 & 80 & 0.3149 & 0.387 & 10 \\
\hline 101 & 60 & 0.2331 & 0.511 & 31.6 & 139 & 100 & 0.3199 & 0.29 & 10 \\
\hline 102 & 80 & 0.2361 & 0.456 & 31.6 & 140 & 120 & 0.3256 & 0.203 & 10 \\
\hline 103 & 100 & 0.2398 & 0.37 & 31.6 & 141 & 25 & 0.3051 & 0.6 & 31.6 \\
\hline 104 & 120 & 0.2441 & 0.28 & 31.6 & 142 & 40 & 0.3072 & 0.55 & 31.6 \\
\hline 105 & 60 & 0.2331 & 0.579 & 100 & 143 & 60 & 0.3107 & 0.5 & 31.6 \\
\hline 106 & 80 & 0.2361 & 0.514 & 100 & 144 & 80 & 0.3149 & 0.431 & 31.6 \\
\hline 107 & 100 & 0.2398 & 0.452 & 100 & 145 & 100 & 0.3199 & 0.352 & 31.6 \\
\hline 108 & 120 & 0.2441 & 0.368 & 100 & 146 & 120 & 0.3256 & 0.273 & 31.6 \\
\hline 109 & 80 & 0.2361 & 0.593 & 316 & 147 & 60 & 0.3107 & 0.549 & 100 \\
\hline 110 & 100 & 0.2398 & 0.533 & 316 & 148 & 80 & 0.3149 & 0.488 & 100 \\
\hline 111 & 120 & 0.2441 & 0.457 & 316 & 149 & 100 & 0.3199 & 0.422 & 100 \\
\hline 112 & 120 & 0.2441 & 0.553 & 1000 & 150 & 120 & 0.3256 & 0.35 & 100 \\
\hline 113 & 25 & 0.3051 & 0.413 & 0.1 & 151 & 80 & 0.3149 & 0.555 & 316 \\
\hline 114 & 40 & 0.3072 & 0.325 & 0.1 & 152 & 100 & 0.3199 & 0.497 & 316 \\
\hline 115 & 60 & 0.3107 & 0.239 & 0.1 & 153 & 120 & 0.3256 & 0.423 & 316 \\
\hline 116 & 80 & 0.3149 & 0.152 & 0.1 & 154 & 100 & 0.3199 & 0.578 & 1000 \\
\hline 117 & 100 & 0.3199 & 0.07 & 0.1 & 155 & 120 & 0.3256 & 0.512 & 1000 \\
\hline 118 & 25 & 0.3051 & 0.449 & 0.316 & & & & & \\
\hline
\end{tabular}


Table B.6 $\mathrm{CO}_{2}$ partial pressure data from $\mathrm{Xu}$ (2011)

\begin{tabular}{ccccc|ccccc}
\hline$\#$ & $\mathrm{~T}\left({ }^{\circ} \mathrm{C}\right)$ & $w_{M E A}^{*}$ & $\alpha$ & $P_{\mathrm{CO}_{2}}$ & $\#$ & $\mathrm{~T}\left({ }^{\circ} \mathrm{C}\right)$ & $w_{M E A}^{*}$ & $\alpha$ & $P_{\mathrm{CO}_{2}}$ \\
\hline 1 & 100 & 0.29408 & 0.388 & 34 & 19 & 120 & 0.29408 & 0.385 & 108 \\
2 & 100 & 0.2956 & 0.501 & 167 & 20 & 120 & 0.2956 & 0.492 & 387 \\
3 & 100 & 0.29499 & 0.52 & 233 & 21 & 120 & 0.29499 & 0.508 & 512 \\
4 & 100 & 0.38825 & 0.411 & 40 & 22 & 120 & 0.2956 & 0.37 & 63 \\
5 & 100 & 0.39301 & 0.52 & 321 & 23 & 120 & 0.38825 & 0.407 & 176 \\
6 & 101 & 0.29529 & 0.475 & 95 & 24 & 120 & 0.39301 & 0.509 & 703 \\
7 & 101.1 & 0.29529 & 0.315 & 12 & 25 & 120.4 & 0.29529 & 0.314 & 50 \\
8 & 109 & 0.29529 & 0.314 & 36 & 26 & 121 & 0.29529 & 0.467 & 283 \\
9 & 110 & 0.29861 & 0.424 & 138 & 27 & 121.8 & 0.29529 & 0.314 & 45 \\
10 & 110 & 0.29861 & 0.424 & 123 & 28 & 129 & 0.29408 & 0.382 & 176 \\
11 & 110 & 0.29408 & 0.387 & 61 & 29 & 129.9 & 0.29529 & 0.313 & 77 \\
12 & 110 & 0.38825 & 0.41 & 98 & 30 & 130 & 0.29861 & 0.419 & 352 \\
13 & 110 & 0.39301 & 0.516 & 477 & 31 & 130 & 0.29861 & 0.421 & 292 \\
14 & 111 & 0.29529 & 0.472 & 171 & 32 & 130 & 0.29529 & 0.461 & 448 \\
15 & 111.3 & 0.29529 & 0.315 & 22 & 33 & 130 & 0.38825 & 0.403 & 316 \\
16 & 120 & 0.29861 & 0.422 & 237 & 34 & 130 & 0.39301 & 0.501 & 996 \\
17 & 120 & 0.29861 & 0.423 & 192 & 35 & 131 & 0.29408 & 0.383 & 153 \\
18 & 120 & 0.29408 & 0.387 & 62 & 36 & 131.9 & 0.29529 & 0.313 & 73 \\
\hline
\end{tabular}

Table $\mathrm{B} .7 \mathrm{CO}_{2}$ partial pressure data from Ma'mun et al. (2005)

\begin{tabular}{ccccc|ccccc}
\hline$\#$ & $\mathrm{~T}\left({ }^{\circ} \mathrm{C}\right)$ & $w_{\mathrm{MEA}}^{*}$ & $\alpha$ & $P_{\mathrm{CO}_{2}}$ & $\#$ & $\mathrm{~T}\left({ }^{\circ} \mathrm{C}\right)$ & $w_{\mathrm{MEA}}^{*}$ & $\alpha$ & $P_{\mathrm{CO}_{2}}$ \\
\hline 1 & 120 & 0.3 & 0.155 & 7.354 & 11 & 120 & 0.3 & 0.3125 & 51.82 \\
2 & 120 & 0.3 & 0.1766 & 9.314 & 12 & 120 & 0.3 & 0.3191 & 58.57 \\
3 & 120 & 0.3 & 0.1843 & 9.045 & 13 & 120 & 0.3 & 0.3298 & 62.88 \\
4 & 120 & 0.3 & 0.2085 & 15.51 & 14 & 120 & 0.3 & 0.3424 & 77.59 \\
5 & 120 & 0.3 & 0.2326 & 19.62 & 15 & 120 & 0.3 & 0.3424 & 74.95 \\
6 & 120 & 0.3 & 0.2381 & 25.2 & 16 & 120 & 0.3 & 0.35 & 83.61 \\
7 & 120 & 0.3 & 0.256 & 27.71 & 17 & 120 & 0.3 & 0.3594 & 92.79 \\
8 & 120 & 0.3 & 0.2901 & 39.18 & 18 & 120 & 0.3 & 0.3882 & 137.9 \\
9 & 120 & 0.3 & 0.2967 & 40.4 & 19 & 120 & 0.3 & 0.4182 & 191.9 \\
10 & 120 & 0.3 & 0.3004 & 43.49 & & & & & \\
\hline
\end{tabular}

\section{B.2. VLE Data for MEA- $\mathrm{H}_{2} \mathrm{O}$ System}

Table B.8. Binary VLE data from Cai et al. (1996)

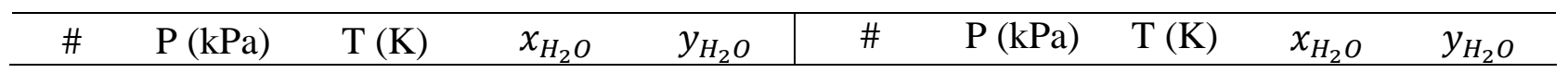




\begin{tabular}{ccccc|ccccc}
\hline 1 & 101.33 & 443.38 & 0 & 0 & 16 & 66.66 & 420.53 & 0.0967 & 0.3589 \\
2 & 101.33 & 431.85 & 0.0736 & 0.3411 & 17 & 66.66 & 416.60 & 0.1332 & 0.4945 \\
3 & 101.33 & 424.76 & 0.1322 & 0.5129 & 18 & 66.66 & 413.33 & 0.1695 & 0.5813 \\
4 & 101.33 & 420.42 & 0.1799 & 0.6252 & 19 & 66.66 & 407.18 & 0.2107 & 0.6781 \\
5 & 101.33 & 415.92 & 0.2522 & 0.7344 & 20 & 66.66 & 403.30 & 0.2589 & 0.7537 \\
6 & 101.33 & 410.94 & 0.2948 & 0.7878 & 21 & 66.66 & 395.37 & 0.3362 & 0.8425 \\
7 & 101.33 & 406.83 & 0.3322 & 0.8312 & 22 & 66.66 & 391.37 & 0.3861 & 0.8848 \\
8 & 101.33 & 394.88 & 0.4694 & 0.9146 & 23 & 66.66 & 385.73 & 0.4477 & 0.9210 \\
9 & 101.33 & 393.25 & 0.5080 & 0.9293 & 24 & 66.66 & 380.82 & 0.5419 & 0.9542 \\
10 & 101.33 & 387.85 & 0.6125 & 0.9596 & 25 & 66.66 & 375.17 & 0.6359 & 0.9732 \\
11 & 101.33 & 378.37 & 0.8238 & 0.9883 & 26 & 66.66 & 367.96 & 0.8363 & 0.9921 \\
12 & 101.33 & 376.88 & 0.8615 & 0.9914 & 27 & 66.66 & 365.60 & 0.8550 & 0.9937 \\
13 & 101.33 & 374.09 & 0.9517 & 0.9974 & 28 & 66.66 & 362.81 & 0.9509 & 0.9982 \\
14 & 101.33 & 373.15 & 1 & 1 & 29 & 66.66 & 361.75 & 1 & 1 \\
15 & 66.66 & 431.39 & 0 & 0 & & & & & \\
\hline
\end{tabular}

Table B.9. Binary VLE data from Tochigi et al. (1999)

\begin{tabular}{ccccc|ccccc}
\hline$\#$ & $\mathrm{P}(\mathrm{kPa})$ & $\mathrm{T}(\mathrm{K})$ & $x_{\mathrm{H}_{2} \mathrm{O}}$ & $y_{\mathrm{H}_{2} \mathrm{O}}$ & $\#$ & $\mathrm{P}(\mathrm{kPa})$ & $\mathrm{T}(\mathrm{K})$ & $x_{\mathrm{H}_{2} \mathrm{O}}$ & $y_{\mathrm{H}_{2} \mathrm{O}}$ \\
\hline 1 & 4.02 & 363.15 & 0 & 0 & 7 & 40.90 & 363.15 & 0.645 & 0.975 \\
2 & 8.03 & 363.15 & 0.112 & 0.560 & 8 & 54.13 & 363.15 & 0.795 & 0.991 \\
3 & 14.05 & 363.15 & 0.240 & 0.796 & 9 & 57.90 & 363.15 & 0.841 & 0.994 \\
4 & 18.59 & 363.15 & 0.326 & 0.869 & 10 & 68.15 & 363.15 & 0.974 & 0.999 \\
5 & 26.82 & 363.15 & 0.466 & 0.931 & 11 & 70.07 & 363.15 & 1 & 1 \\
6 & 32.02 & 363.15 & 0.529 & 0.952 & & & & & \\
\hline
\end{tabular}

\section{B.3 Heat of Absorption}

All data are given for $w_{M E A}^{*}=0.3$. The values are differential heat of absorption, and all are negative values, indicating that the absorption of $\mathrm{CO}_{2}$ is an exothermic process.

Table B.10 Heat of absorption data (Kim et al., 2014)

\begin{tabular}{cccc|cccc}
\hline$\#$ & $\mathrm{~T}\left({ }^{\circ} \mathrm{C}\right)$ & $\alpha$ & $-\Delta H_{a b s}\left(\frac{k J}{m o l ~ C O_{2}}\right)$ & & $\begin{array}{c}\mathrm{T} \\
\left({ }^{\circ} \mathrm{C}\right)\end{array}$ & $\alpha$ & $-\Delta H_{a b s}\left(\frac{k J}{m o l ~ C O_{2}}\right)$ \\
\hline 1 & 40 & 0.06 & 84.03 & 15 & 80 & 0.28 & 88.05 \\
2 & 40 & 0.15 & 84.36 & 16 & 80 & 0.36 & 88.27 \\
3 & 40 & 0.23 & 85.44 & 17 & 80 & 0.45 & 85.55 \\
4 & 40 & 0.31 & 85.36 & 18 & 80 & 0.50 & 73.71 \\
5 & 40 & 0.39 & 85.76 & 19 & 80 & 0.55 & 58.94 \\
6 & 40 & 0.44 & 81.55 & 20 & 80 & 0.58 & 51.05 \\
7 & 40 & 0.51 & 71.30 & 21 & 120 & 0.07 & 99.08
\end{tabular}




\begin{tabular}{cccc|cccc}
8 & 40 & 0.56 & 48.60 & 22 & 120 & 0.16 & 96.95 \\
9 & 40 & 0.61 & 40.24 & 23 & 120 & 0.25 & 100.77 \\
10 & 40 & 0.65 & 36.67 & 24 & 120 & 0.33 & 99.28 \\
11 & 40 & 0.69 & 35.51 & 25 & 120 & 0.40 & 95.55 \\
12 & 80 & 0.06 & 88.55 & 26 & 120 & 0.46 & 86.05 \\
13 & 80 & 0.14 & 88.25 & 27 & 120 & 0.48 & 79.74 \\
14 & 80 & 0.20 & 88.52 & & & & \\
\hline
\end{tabular}

\section{B.3 Heat Capacity}

Table B.10 Heat capacity data (Weiland et al., 1997) for a temperature of $25^{\circ} \mathrm{C}$.

\begin{tabular}{cccc|cccc}
\hline$\#$ & $w_{M E A}^{*}$ & $\alpha$ & $C_{p}\left(\frac{k J}{k g-K}\right)$ & $\#$ & $w_{M E A}^{*}$ & $\alpha$ & $C_{p}\left(\frac{k J}{k g-K}\right)$ \\
\hline 1 & 0.1 & 0 & 4.061 & 13 & 0.3 & 0 & 3.734 \\
2 & 0.1 & 0.05 & 4.015 & 14 & 0.3 & 0.10 & 3.656 \\
3 & 0.1 & 0.20 & 3.917 & 15 & 0.3 & 0.20 & 3.570 \\
4 & 0.1 & 0.30 & 3.915 & 16 & 0.3 & 0.30 & 3.457 \\
5 & 0.1 & 0.40 & 3.891 & 17 & 0.3 & 0.40 & 3.418 \\
6 & 0.1 & 0.50 & 3.857 & 18 & 0.3 & 0.50 & 3.359 \\
7 & 0.2 & 0 & 3.911 & 19 & 0.4 & 0 & 3.634 \\
8 & 0.2 & 0.10 & 3.823 & 20 & 0.4 & 0.10 & 3.508 \\
9 & 0.2 & 0.20 & 3.766 & 21 & 0.4 & 0.20 & 3.343 \\
10 & 0.2 & 0.30 & 3.67 & 22 & 0.4 & 0.30 & 3.238 \\
11 & 0.2 & 0.40 & 3.648 & 23 & 0.4 & 0.40 & 3.163 \\
12 & 0.2 & 0.50 & 3.597 & 24 & 0.4 & 0.50 & 3.109 \\
\hline
\end{tabular}




\section{Appendix C. FORTRAN User Models Described in Work}

The FORTRAN models included in this appendix are adapted from templates provided by Aspen Tech $^{\circledR}$, which are described in the user models manual (Aspen Plus documentation, 2013d).

\section{C.1 Viscosity Model Subroutine}

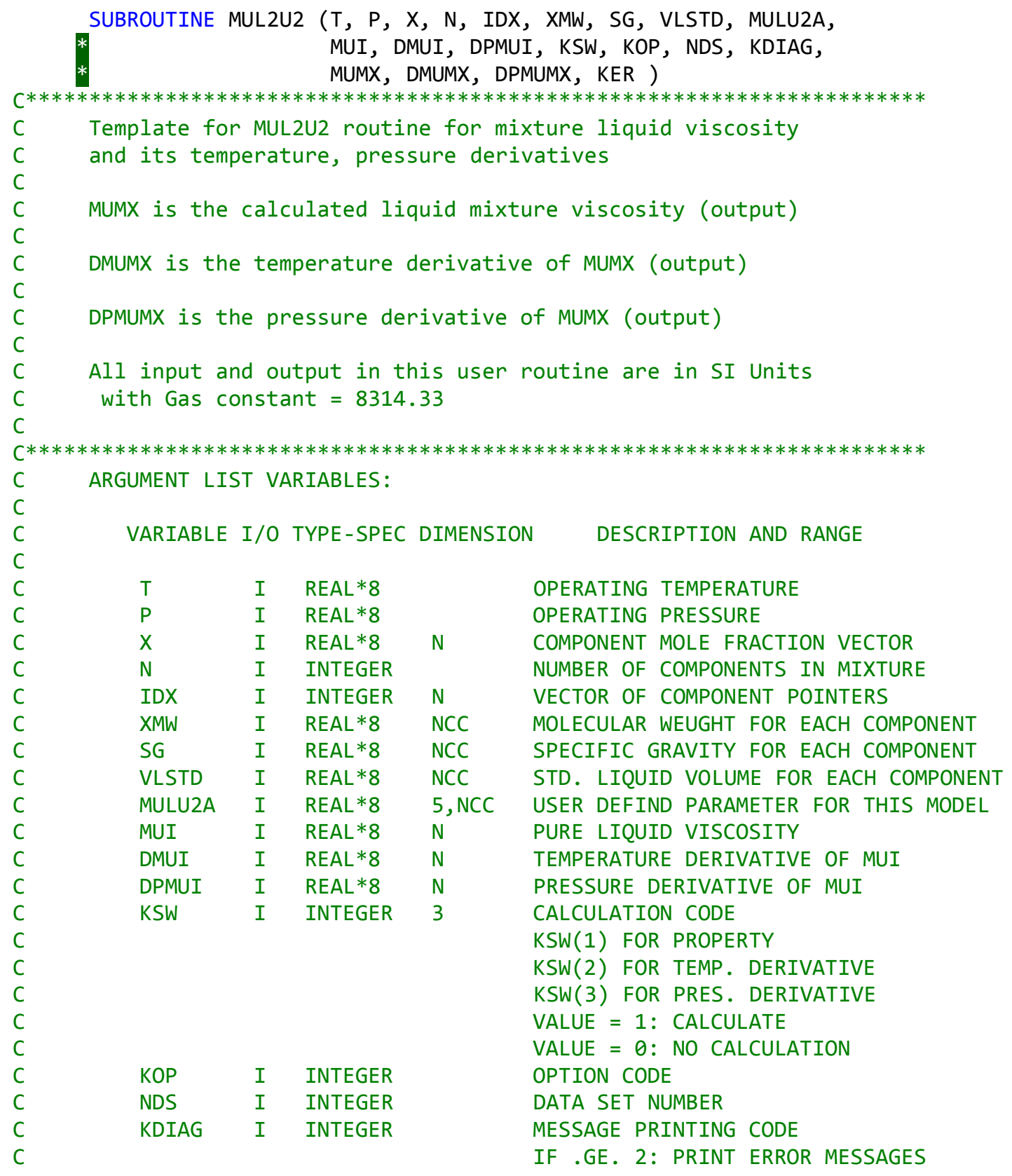




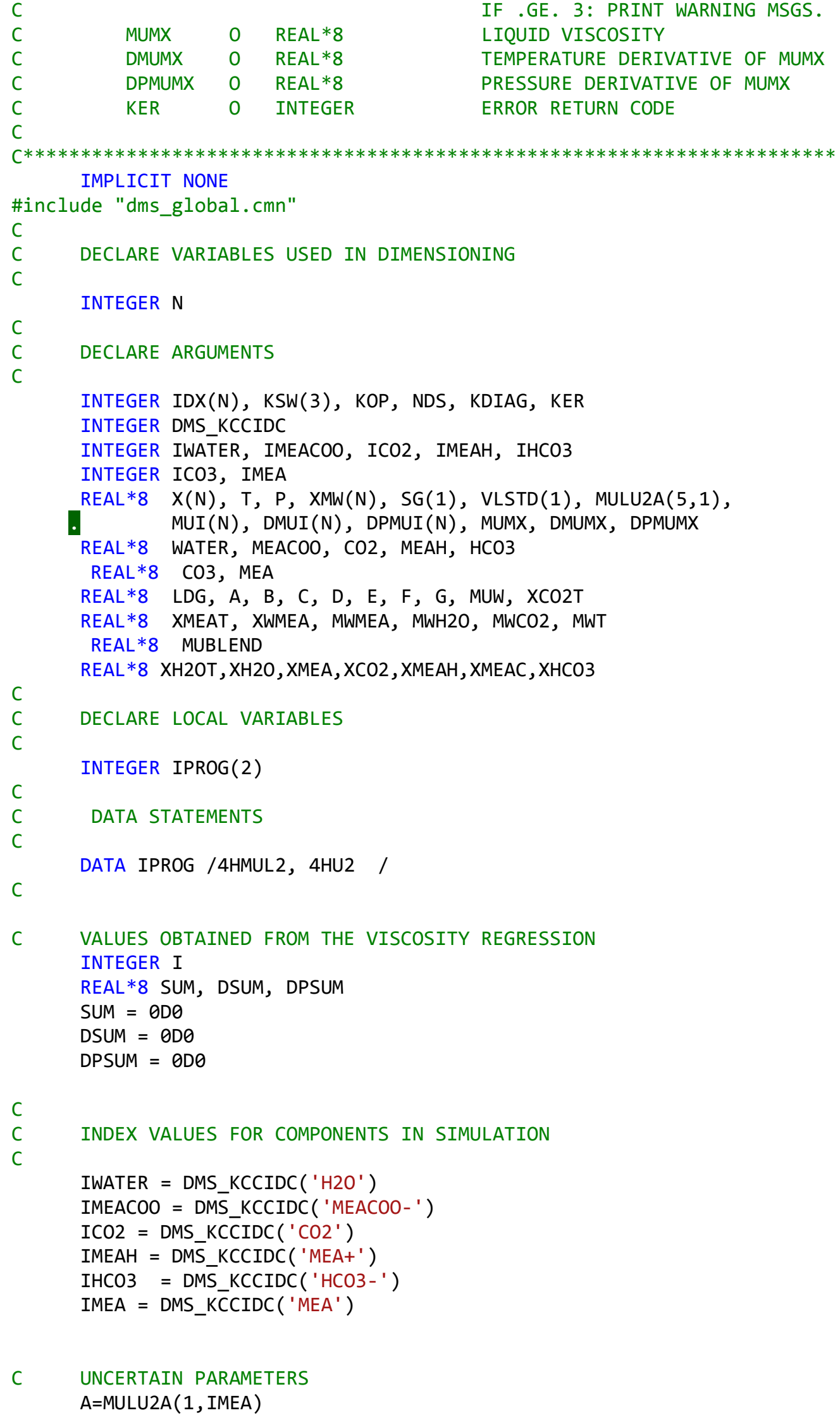




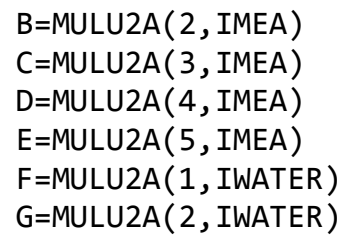

MUW $=1.002$

MUW $=$ MUW*10**(1.3272*(293.15-T-0.001053*(T-293.15)**2)/(T-168.15))

C INITIALIZE AND SUBSEQUENTLY CALCULATE COMPONENT MOLE FRACTIONS

$\mathrm{XH} 2 \mathrm{O}=0$

$\mathrm{XMEA}=0$

$X C O 2=0$

$\mathrm{XMEAH}=0$

$\mathrm{XMEAC}=0$

$\mathrm{XHCO} 3=0$

DO $I=1, N$

IF (IDX(I). EQ. IWATER) XH2O $=X H 2 O+X(I)$

IF (IDX (I). EQ. IMEA) XMEA $=X M E A+X(I)$

IF (IDX(I). EQ. ICO2) XCO2=XCO2+X(I)

IF (IDX(I) .EQ. IMEAH) XMEAH $=X M E A H+X(I)$

IF ( IDX (I).EQ. IHCO3) XHCO3=XHCO3+X(I)

IF $(\operatorname{IDX}(\mathrm{I}) \cdot \mathrm{EQ} \cdot \mathrm{IMEACOO}) \mathrm{XMEAC}=\mathrm{XMEAC}+\mathrm{X}(\mathrm{I})$

END DO

C APPARENT COMPONENT CALCULATIONS

C

$\mathrm{XCO2T}=\mathrm{XMEAC}+\mathrm{XHCO3}+\mathrm{XCO2}$

XMEAT $=$ XMEAC + XMEAH + XMEA

$\mathrm{XH} 2 \mathrm{OT}=\mathrm{XH} 2 \mathrm{O}+\mathrm{XHCO} 3$

C

C

C

AMINE MASS FRACTION AND LOADING CALCULATIONS

MWMEA $=61.08308 \mathrm{D0}$

MWCO2 $=44.0095 \mathrm{DO}$

$M W H 20=18.01528 D 0$

LDG $=$ XCO2T $/$ XMEAT

MWT $=$ XMEAT*MWMEA $+X H 20 * M W H 2 O$

XWMEA $=(($ XMEAT $*$ MWMEA $) /$ MWT $) * 100$

C

C

$\mathrm{C}$

C

VISCOSITY CALCULATION

MUBLEND $=(A *$ XWMEA $+B) * T+(C *$ XWMEA $+D)$

MUBLEND $=$ MUBLEND $*($ LDG* $(E * X W M E A+F * T+G)+1) * X W M E A$

MUBLEND $=$ MUBLEND $/ T^{* * 2}$

MUBLEND $=$ DEXP(MUBLEND)

MUMX $=$ MUBLEND*MUW $/ 1000$

$C$ viscosity calculated in $\mathrm{Pa}-\mathrm{S}$

$\mathrm{C}$

C MUMX 


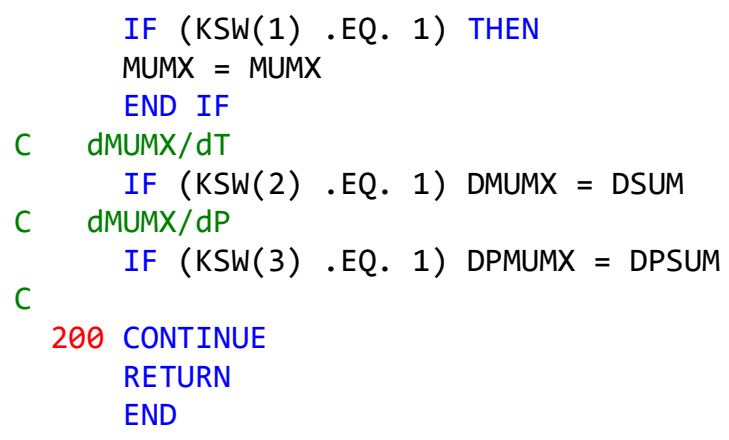

\section{C.2 Molar Volume Model Subroutine}

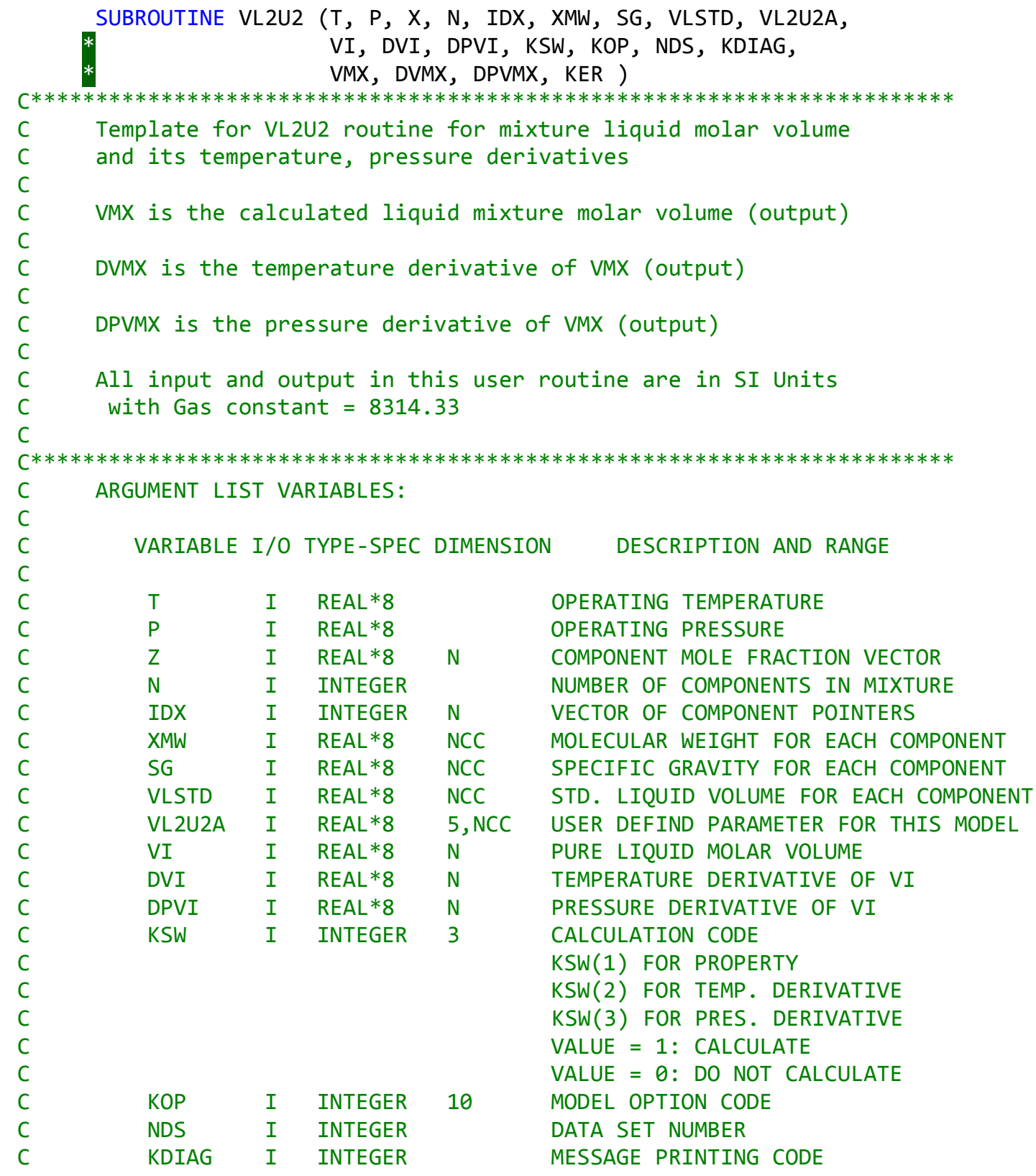

ARGUMENT LIST VARIABLES :

VARIABLE I/O TYPE-SPEC DIMENSION

DESCRIPTION AND RANGE

$\begin{array}{llll}\text { T } & \text { I } & \text { REAL*8 } \\ \text { P } & \text { I } & \text { REAL*8 } & \\ \text { Z } & \text { I } & \text { REAL*8 } & \text { N } \\ \text { N } & \text { I } & \text { INTEGER } & \\ \text { IDX } & \text { I } & \text { INTEGER } & \text { N } \\ \text { XMW } & \text { I } & \text { REAL*8 } & \text { NCC } \\ \text { SG } & \text { I } & \text { REAL*8 } & \text { NCC } \\ \text { VLSTD } & \text { I } & \text { REAL*8 } & \text { NCC } \\ \text { VL2U2A } & \text { I } & \text { REAL*8 } & 5 \text {, NCC } \\ \text { VI } & \text { I } & \text { REAL*8 } & \text { N } \\ \text { DVI } & \text { I } & \text { REAL*8 } & \text { N } \\ \text { DPVI } & \text { I } & \text { REAL*8 } & \text { N } \\ \text { KSW } & \text { I } & \text { INTEGER } & 3\end{array}$

OPERATING TEMPERATURE OPERATING PRESSURE COMPONENT MOLE FRACTION VECTOR NUMBER OF COMPONENTS IN MIXTURE VECTOR OF COMPONENT POINTERS MOLECULAR WEIGHT FOR EACH COMPONENT SPECIFIC GRAVITY FOR EACH COMPONENT STD. LIQUID VOLUME FOR EACH COMPONENT USER DEFIND PARAMETER FOR THIS MODEL PURE LIQUID MOLAR VOLUME TEMPERATURE DERIVATIVE OF VI PRESSURE DERIVATIVE OF VI CALCULATION CODE KSW(1) FOR PROPERTY KSW(2) FOR TEMP. DERIVATIVE KSW(3) FOR PRES. DERIVATIVE VALUE $=1:$ CALCULATE VALUE $=0:$ DO NOT CALCULATE $\begin{array}{llll}\text { KOP } & \text { I } & \text { INTEGER } & 10 \\ \text { NDS } & \text { I } & \text { INTEGER } & \\ \text { KDIAG } & \text { I } & \text { INTEGER } & \end{array}$ MODEL OPTION CODE DATA SET NUMBER MESSAGE PRINTING CODE 


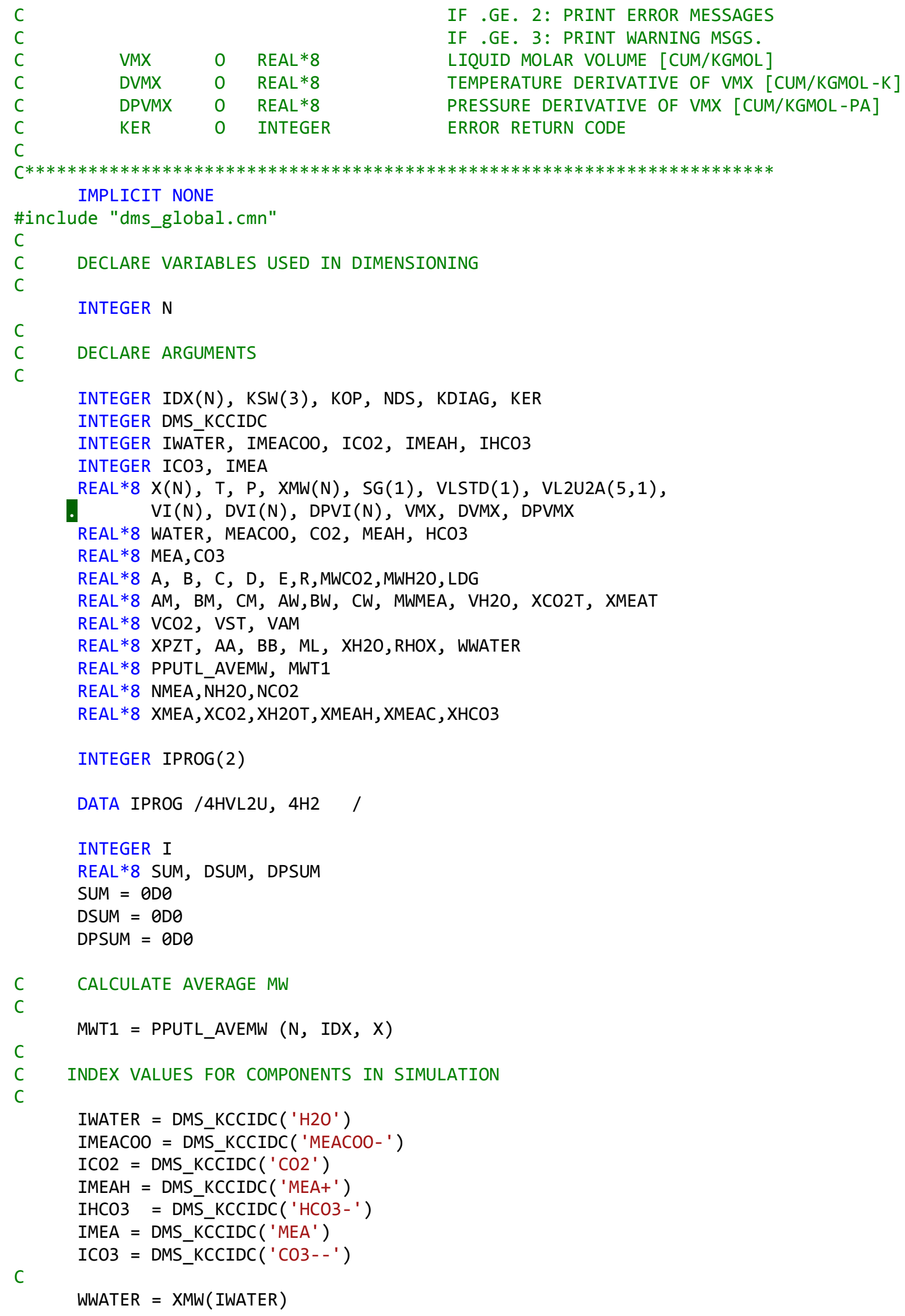


$\mathrm{XH} 2 \mathrm{O}=0$

$\mathrm{XMEAH}=0$

$X M E A C=0$

$\mathrm{XHCO} 3=0$

DO $I=1, N$

IF (IDX(I). EQ. IWATER) $X H 2 O=X H 2 O+X(I)$

IF (IDX(I). EQ. IMEACOO) XMEAC $=X M E A C+X(I)$

IF (IDX(I). EQ. ICO2) XCO2 $=X C O 2+X(I)$

IF (IDX(I). EQ. IMEAH) XMEAH $=X M E A H+X(I)$

IF (IDX(I). EQ. IHCO3) XHCO3 $=X \mathrm{HCO} 3+X(I)$

IF $(\operatorname{IDX}(I) . E Q$. IMEA) XMEA $=X M E A+X(I)$

END DO

C UNCERTAIN PARAMETERS

$\mathrm{A}=\mathrm{VL} 2 \mathrm{U} 2 \mathrm{~A}(1, \mathrm{IMEA})$

$B=V L 2 U 2 A(2$, IMEA $)$

$C=\operatorname{VL} 2 U 2 A(3$, IMEA $)$

$\mathrm{D}=\mathrm{VL} 2 \mathrm{U} 2 \mathrm{~A}(4, \mathrm{IMEA})$

$E=\operatorname{VL} 2 U 2 A(5$, IMEA $)$

C CONSTANTS

$\mathrm{AM}=-0.000000535162$

$\mathrm{BM}=-0.000451417$

$\mathrm{CM}=1.19451$

$\mathrm{AW}=-0.00000324839$

$\mathrm{BW}=0.00165311$

$\mathrm{CW}=0.793041$

C PURE COMPONENT MOLAR VOLUME

$\mathrm{VH} 20=18.01528 /(\mathrm{AW} * \mathrm{~T} * * 2+\mathrm{BW} * \mathrm{~T}+\mathrm{CW})$

$V A M=61.08308 /(A M * T * * 2+B M * T+C M)$

C LOADING CALCULATIONS

$\mathrm{XH} 2 \mathrm{OT}=\mathrm{XH} 2 \mathrm{O}+\mathrm{XHCO}$

$\mathrm{XCO2T}=\mathrm{XMEAC}+\mathrm{XHCO3}+\mathrm{XCO} 2$

XMEAT $=$ XMEAC + XMEAH + XMEA

MWMEA $=61.08308 \mathrm{D} 0$

MWCO2 $=44.0095 \mathrm{DO}$

$\mathrm{MWH} 20=18.01528 \mathrm{D0}$

$\mathrm{R}=($ MWMEA $*$ XMEAT $) /($ MWMEA $*$ XMEAT + MWH2O*XH2OT $)$

$\mathrm{LDG}=\mathrm{XCO} 2 \mathrm{~T} / \mathrm{XMEAT}$

NMEA $=(1+\mathrm{LDG}+($ MWMEA $/ M W H 20) *(1-\mathrm{R}) / \mathrm{R}) * *(-1)$

$\mathrm{NCO} 2=\mathrm{NMEA} *$ LDG

$\mathrm{NH} 2 \mathrm{O}=1-\mathrm{NMEA}-\mathrm{NCO} 2$

C MOLAR VOLUME

$\mathrm{VMX}=\mathrm{NMEA} * \mathrm{VAM}+\mathrm{NH} 2 \mathrm{O} * \mathrm{VH} 2 \mathrm{O}+\mathrm{NCO} 2 * A+\mathrm{NMEA} * \mathrm{NH} 2 \mathrm{O} *(\mathrm{~B}+\mathrm{C} * \mathrm{NMEA})$

$\mathrm{VMX}=\mathrm{VMX}+\mathrm{NMEA} * \mathrm{NCO} 2 *(\mathrm{D}+\mathrm{E} * \mathrm{NMEA})$

C VMX

IF $(\mathrm{KSW}(1) \cdot \mathrm{EQ} \cdot 1) \mathrm{VMX}=\mathrm{VMX} / 1000 \mathrm{D0}$ 
C dVMX/dT

$\mathrm{IF}(\mathrm{KSW}(2) . \mathrm{EQ} \cdot 1) \mathrm{DVMX}=\mathrm{DSUM}$

C $\quad d V M X / d P$

IF $(K S W(3) \cdot E Q \cdot 1)$ DPVMX = DPSUM

200 CONTINUE

RETURN

END

\section{C.3 Surface Tension Model Subroutine}

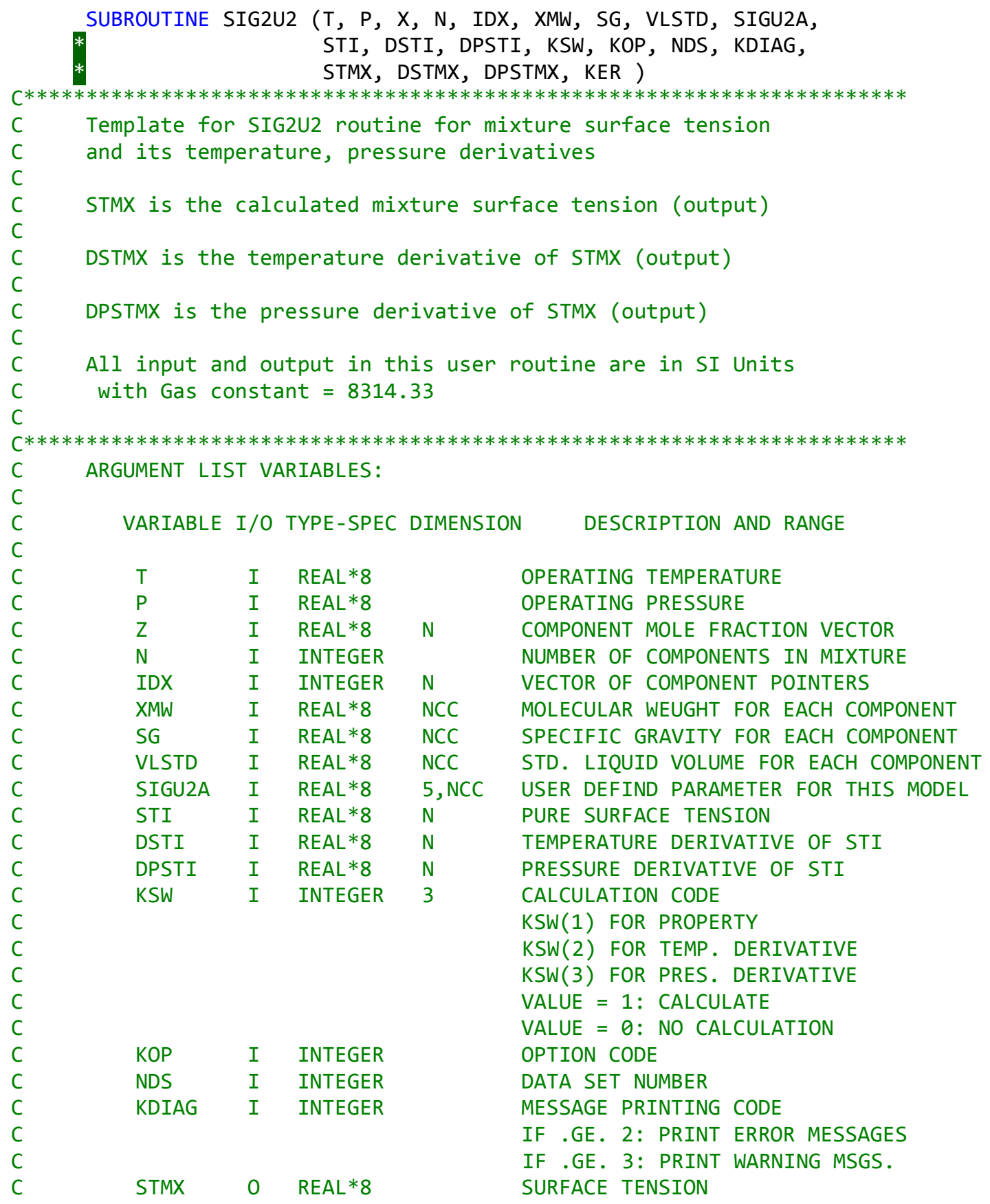




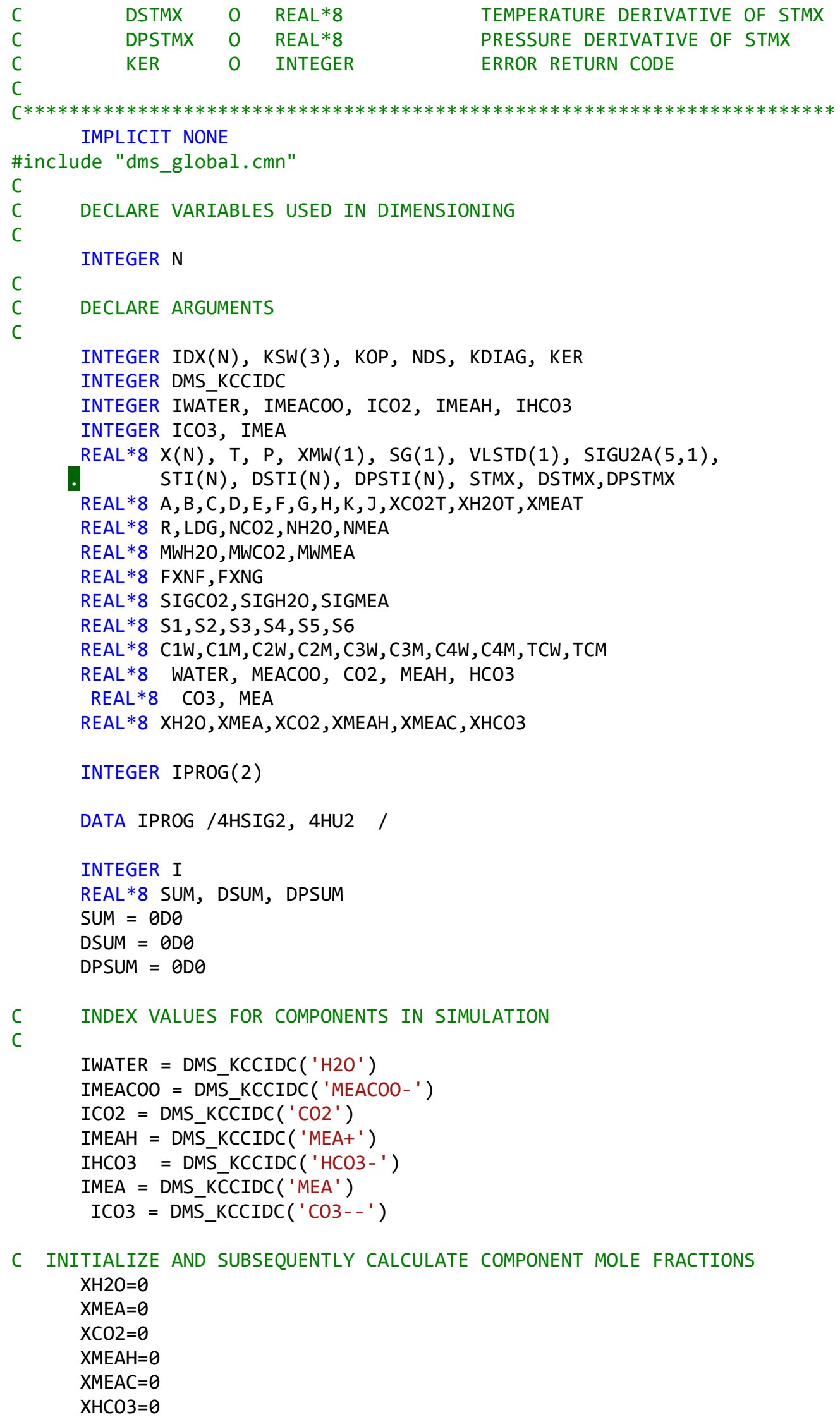


DO $I=1, N$

IF (IDX(I). EQ. IWATER) $X H 2 O=X H 2 O+X(I)$

IF (IDX(I). EQ. IMEACOO) XMEAC $=X M E A C+X(I)$

IF (IDX(I). EQ. ICO2) XCO2=XCO2+X(I)

IF (IDX(I). EQ. IMEAH) XMEAH $=X M E A H+X(I)$

IF (IDX(I). EQ. IHCO3) XHCO3 $=X \mathrm{XHCO}+X(I)$

IF (IDX(I). EQ. IMEA) XMEA=XMEA+X(I)

END DO

C UNCERTAIN PARAMETERS

$A=\operatorname{SIGU2A}(1$, IMEA $)$

$B=\operatorname{SIGU} 2 A(2, I M E A)$

$C=\operatorname{SIGU} 2 A(3$, IMEA $)$

$D=\operatorname{SIGU} 2 A(4$, IMEA $)$

$\mathrm{E}=\mathrm{SIGU} 2 \mathrm{~A}(5, \mathrm{IMEA})$

$\mathrm{F}=\mathrm{SIGU} 2 \mathrm{~A}(1, \mathrm{IWATER})$

$\mathrm{G}=\mathrm{SIGU} 2 \mathrm{~A}(2$, IWATER $)$

$\mathrm{H}=\mathrm{SIGU} 2 \mathrm{~A}(3$, IWATER )

$K=\operatorname{SIGU2A}(4$, IWATER $)$

$J=S I G U 2 A(5$, IWATER $)$

C CONSTANTS

MWMEA $=61.08308$

MWCO2 $=44.0095$

$\mathrm{MWH} 2 \mathrm{O}=18.01528$

$\mathrm{S} 1=-5.987$

$\mathrm{S} 2=3.7699$

$\mathrm{S} 3=-0.43164$

$\mathrm{S} 4=0.018155$

$\mathrm{S} 5=-0.01207$

$\mathrm{S} 6=0.002119$

$\mathrm{C} 1 \mathrm{~W}=0.18548$

$\mathrm{C} 1 \mathrm{M}=0.09945$

$\mathrm{C} 2 \mathrm{~W}=2.717$

$\mathrm{C} 2 \mathrm{M}=1.067$

$\mathrm{C} 3 \mathrm{~W}=-3.554$

$\mathrm{C} 3 \mathrm{M}=0$

$\mathrm{C} 4 \mathrm{~W}=2.047$

$\mathrm{C} 4 \mathrm{M}=0$

$\mathrm{TCW}=647.13$

$\mathrm{TCM}=614.45$

C MOLE FRACTION CALCULATIONS

$\mathrm{XCO} 2 \mathrm{~T}=\mathrm{XMEAC}+\mathrm{XHCO}+\mathrm{XCO} 2$

$X M E A T=X M E A C+X M E A H+X M E A$

$\mathrm{XH} 2 \mathrm{OT}=\mathrm{XH} 2 \mathrm{O}+\mathrm{XHCO} 3$

$\mathrm{R}=($ MWMEA $* X M E A T) /($ MWMEA $* X M E A T+M W H 2 O * X H 2 O T)$

LDG $=X C 02 T / X M E A T$

NMEA $=(1+\mathrm{LDG}+($ MWMEA $/ \mathrm{MWH} 20) *(1-\mathrm{R}) / \mathrm{R}) * *(-1)$

$\mathrm{NCO} 2=\mathrm{NMEA} *$ LDG

$\mathrm{NH} 2 \mathrm{O}=1-\mathrm{NMEA}-\mathrm{NCO} 2$

C ASSEMBLY OF THE SURFACE TENSION MODEL 
$\mathrm{FXNF}=\mathrm{A}+\mathrm{B} * \mathrm{LDG}+\mathrm{C} * \mathrm{LDG} * * 2+\mathrm{D} * \mathrm{R}+\mathrm{E} * \mathrm{R} * * 2$

$\mathrm{FXNG}=\mathrm{F}+\mathrm{G} * \mathrm{LDG}+\mathrm{H} * \mathrm{LDG} * * 2+\mathrm{K} * \mathrm{R}+\mathrm{J} * \mathrm{R} * * 2$

$\mathrm{SIGCO} 2=\mathrm{S} 1 * \mathrm{R} * * 2+\mathrm{S} 2 * \mathrm{R}+\mathrm{S} 3+\mathrm{T} *(\mathrm{~S} 4 * \mathrm{R} * * 2+\mathrm{S} 5 * \mathrm{R}+\mathrm{S} 6)$

$\mathrm{SIGH} 20=\mathrm{C} 1 \mathrm{~W} *(1-\mathrm{T} / \mathrm{TCW}) * *(\mathrm{C} 2 \mathrm{~W}+\mathrm{C} 3 \mathrm{~W} *(\mathrm{~T} / \mathrm{TCW})+\mathrm{C} 4 \mathrm{~W} *(\mathrm{~T} / \mathrm{TCW}) * * 2)$

SIGMEA $=C 1 M *(1-\mathrm{T} / \mathrm{TCM}) * *(\mathrm{C} 2 \mathrm{M}+\mathrm{C} 3 \mathrm{M} *(\mathrm{~T} / \mathrm{TCM})+\mathrm{C} 4 \mathrm{M} *(\mathrm{~T} / \mathrm{TCM}) * * 2)$

C The final equation

STMX $=$ SIGH2O+(SIGCO2-SIGH2O) *FXNF*NCO2+(SIGMEA-SIGH2O)*FXNG*NMEA

C STUMX

$\operatorname{IF}(K S W(1) \cdot E Q \cdot 1) \operatorname{STMX}=\operatorname{STMX}$

C dSTMX/dT

IF $(\mathrm{KSW}(2) \cdot \mathrm{EQ} \cdot 1)$ DSTMX = DSUM

C dSTMX/dP

IF $(K S W(3) \cdot E Q \cdot 1)$ DPSTMX = DPSUM

C

200 CONTINUE

RETURN

END

\section{C.4 Reaction Kinetics Subroutine}

\begin{tabular}{|c|c|c|c|c|c|}
\hline SUBROUTINE ACTKIN2 & $\begin{array}{l}(\mathrm{N}, \\
\mathrm{T}, \\
\mathrm{F}, \\
\text { KDIAG, } \\
\text { IHVBAS, } \\
\text { NREAL, } \\
\text { NINTB, } \\
\text { IWORK, }\end{array}$ & $\begin{array}{l}\text { NCOMP, } \\
\text { TLIQ, } \\
\text { X, } \\
\text { STOIC, } \\
\text { HLDVAP, } \\
\text { REAL, } \\
\text { INTB, } \\
\text { NWORK, }\end{array}$ & $\begin{array}{l}\text { NR, } \\
\text { TVAP, } \\
\text { Y, } \\
\text { IHLBAS, } \\
\text { TIMVAP, } \\
\text { RATES, } \\
\text { NREALB, } \\
\text { WORK) }\end{array}$ & $\begin{array}{l}\text { NRL, } \\
\text { P, } \\
\text { IDX, } \\
\text { HLDLIQ, } \\
\text { NINT, } \\
\text { RATEL, } \\
\text { REALB, }\end{array}$ & $\begin{array}{l}\text { NRV, } \\
\text { PHFRAC, } \\
\text { NBOPST, } \\
\text { TIMLIQ, } \\
\text { INT, } \\
\text { RATEV, } \\
\text { NIWORK, }\end{array}$ \\
\hline
\end{tabular}

$C$
$C$
$C$
$C$
$C$
$C$
$C$
$C$
$C$
$C$
$C$
$C$
$C$
$C$
$C$
$C$
$C$
$C$
$C$
$C$
$C$
$C$
$C$
$C$

VARIABLES IN ARGUMENT LIST

VARIABLE I/O TYPE DIMENSION $\mathrm{N} I \mathrm{I}$

NCOMP I I

$\begin{array}{lll}\text { NR } & \text { I } & \text { I }\end{array}$

NRL

I I

NRV

I I

$\mathrm{T}$

TLIQ

I $\quad \mathrm{R}$

\section{TVAP}

I

P

PHFRAC

DIMENSION
-
-
-
3

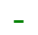

$-$

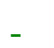

$-$

3

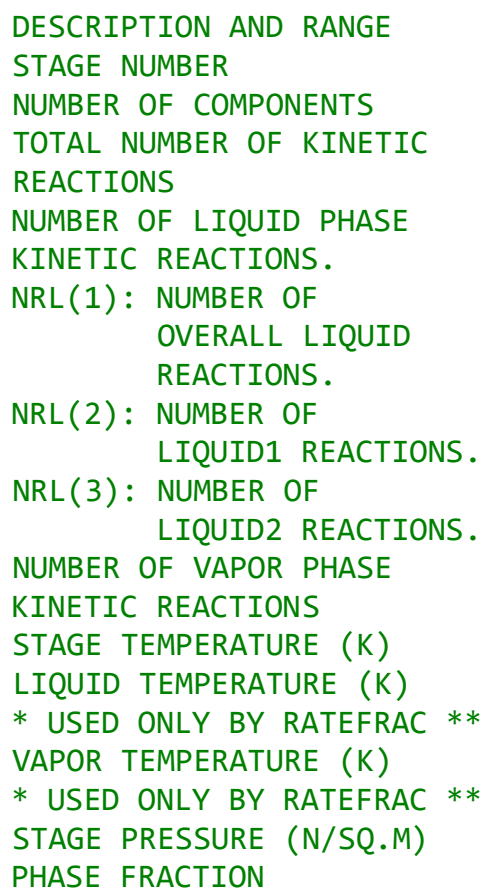




\begin{tabular}{|c|c|c|c|c|c|}
\hline C & & & & & $\operatorname{PHFRAC}(1):$ VAPOR FRACTION \\
\hline C & & & & & PHFRAC (2): LIQUID1 FRACTIO \\
\hline C & & & & & PHFRAC(3): LIQUID2 FRACTIO \\
\hline C & $\mathrm{F}$ & I & $\mathrm{R}$ & - & TOTAL FLOW ON STAGE \\
\hline C & & & & & (VAPOR+LIQUID) (KMOL/SEC) \\
\hline C & $\mathrm{X}$ & $\mathrm{I}$ & $\mathrm{R}$ & NCOMP, 3 & LIQUID MOLE FRACTION \\
\hline C & $\mathrm{Y}$ & I & $\mathrm{R}$ & NCOMP & VAPOR MOLE FRACTION \\
\hline C & IDX & I & $\mathrm{I}$ & NCOMP & COMPONENT INDEX VECTOR \\
\hline C & NBOPST & I & $\mathrm{I}$ & 6 & OPTION SET BEAD POINTER \\
\hline C & KDIAG & $\mathrm{I}$ & $\mathrm{I}$ & - & LOCAL DIAGNOSTIC LEVEL \\
\hline C & STOIC & $\mathrm{I}$ & $\mathrm{R}$ & NCOMP, NR & REACTION STOICHIOMETRY \\
\hline $\mathrm{C}$ & IHLBAS & $\mathrm{I}$ & $\mathrm{I}$ & - & BASIS FOR LIQUID \\
\hline $\mathrm{C}$ & & & & & HOLDUP SPECIFICATION \\
\hline $\mathrm{C}$ & & & & & $1:$ VOLUME , 2 :MASS , $3:$ MOLE \\
\hline $\mathrm{C}$ & HLDLIQ & I & $\mathrm{R}$ & - & LIQUID HOLDUP \\
\hline $\mathrm{C}$ & & & & & IHLBAS UNITS \\
\hline $\mathrm{C}$ & & & & & CU.M. \\
\hline $\mathrm{C}$ & & & & & $2 \quad \mathrm{KG}$ \\
\hline $\mathrm{C}$ & & & & & KMOL \\
\hline $\mathrm{C}$ & TIMLIQ & $\mathrm{I}$ & $\mathrm{R}$ & - & LIQUID RESIDENCE TIME \\
\hline $\mathrm{C}$ & & & & & $(\mathrm{SEC})$ \\
\hline $\mathrm{C}$ & IHVBAS & I & $\mathrm{I}$ & - & BASIS FOR VAPOR \\
\hline $\mathrm{C}$ & & & & & HOLDUP SPECIFICATION \\
\hline $\mathrm{C}$ & & & & & $1:$ VOLUME , $2:$ MASS , $3:$ MOLE \\
\hline $\mathrm{C}$ & HLDVAP & $\mathrm{I}$ & $\mathrm{R}$ & - & VAPOR HOLDUP \\
\hline $\mathrm{C}$ & & & & & IHVBAS UNITS \\
\hline $\mathrm{C}$ & & & & & 1 CU.M. \\
\hline $\mathrm{C}$ & & & & & KG \\
\hline $\mathrm{C}$ & & & & & KMOL \\
\hline $\mathrm{C}$ & TIMVAP & $I$ & $\mathrm{R}$ & - & VAPOR RESIDENCE TIME (SEC) \\
\hline $\mathrm{C}$ & NINT & $\mathrm{I}$ & I & - & LENGTH OF INTEGER VECTOR \\
\hline $\mathrm{C}$ & INT & $\mathrm{I} / 0$ & $\mathrm{I}$ & NINT & INTEGER VECTOR \\
\hline $\mathrm{C}$ & NREAL & $\mathrm{I}$ & $\mathrm{I}$ & - & LENGTH OF REAL VECTOR \\
\hline $\mathrm{C}$ & REAL & $\mathrm{I} / 0$ & $\mathrm{R}$ & NREAL & REAL VECTOR \\
\hline $\mathrm{C}$ & RATES & 0 & $\mathrm{R}$ & NCOMP & COMPONENT REACTION RATES \\
\hline C & & & & & $(\mathrm{KMOL} / \mathrm{SEC})$ \\
\hline $\mathrm{C}$ & RATEL & 0 & $\mathrm{R}$ & NRLT & INDIVIDUAL REACTION RATES \\
\hline C & & & & & IN THE LIQUID PHASE \\
\hline $\mathrm{C}$ & & & & & $(\mathrm{KMOL} / \mathrm{SEC})$ \\
\hline $\mathrm{C}$ & & & & & WHAT IS NRLT? \\
\hline $\mathrm{C}$ & & & & & $N R L T=N R L(1)+N R L(2)+N R L(3$ \\
\hline $\mathrm{C}$ & & & & & NRLT IS NOT INCLUDED IN TH \\
\hline $\mathrm{C}$ & & & & & ARGUMENT LIST. \\
\hline $\mathrm{C}$ & & & & & * USED ONLY BY RATEFRAC * \\
\hline $\mathrm{C}$ & RATEV & 0 & $\mathrm{R}$ & NRV & INDIVIDUAL REACTION RATES \\
\hline $\mathrm{C}$ & & & & & IN THE VAPOR PHASE \\
\hline $\mathrm{C}$ & & & & & (KMOL/SEC) \\
\hline $\mathrm{C}$ & & & & & * USED ONLY BY RATEFRAC * \\
\hline $\mathrm{C}$ & NINTB & I & $\mathrm{I}$ & - & LENGTH OF INTEGER VECTOR \\
\hline $\mathrm{C}$ & & & & & (FROM UOS BLOCK) \\
\hline $\mathrm{C}$ & INTB & $\mathrm{I} / 0$ & $\mathrm{I}$ & NINTB & INTEGER VECTOR \\
\hline $\mathrm{C}$ & & & & & (FROM UOS BLOCK) \\
\hline $\mathrm{C}$ & NREALB & $\mathrm{I}$ & $\mathrm{I}$ & - & LENGTH OF REAL VECTOR \\
\hline $\mathrm{C}$ & & & & & (FROM UOS BLOCK) \\
\hline $\mathrm{C}$ & REALB & $\mathrm{I} / 0$ & $\mathrm{R}$ & NREALB & REAL VECTOR \\
\hline $\mathrm{C}$ & & & & & (FROM UOS BLOCK) \\
\hline $\mathrm{C}$ & NIWORK & $\mathrm{I}$ & $\mathrm{I}$ & - & LENGTH OF INTEGER WORK \\
\hline $\mathrm{C}$ & & & & & VECTOR \\
\hline
\end{tabular}




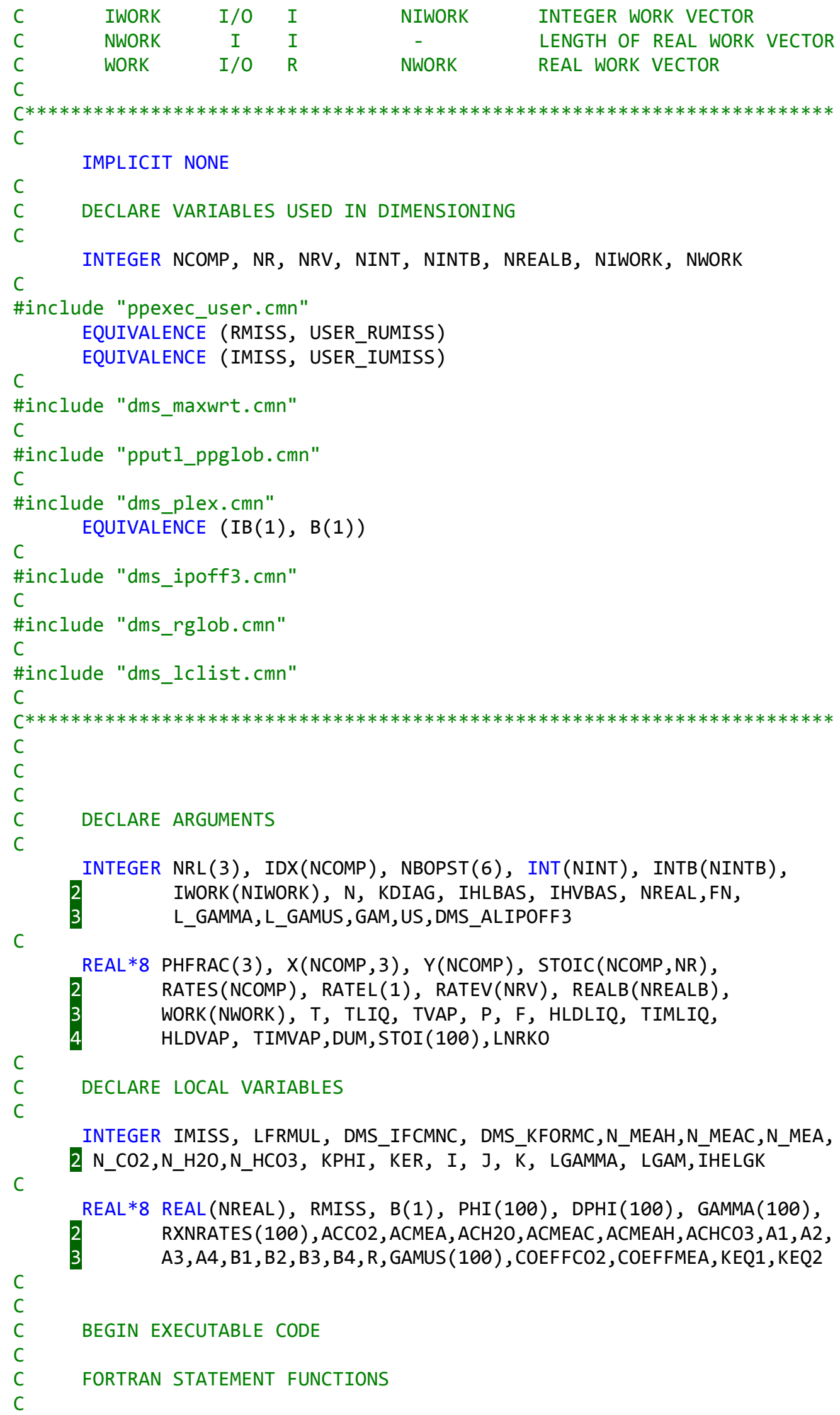




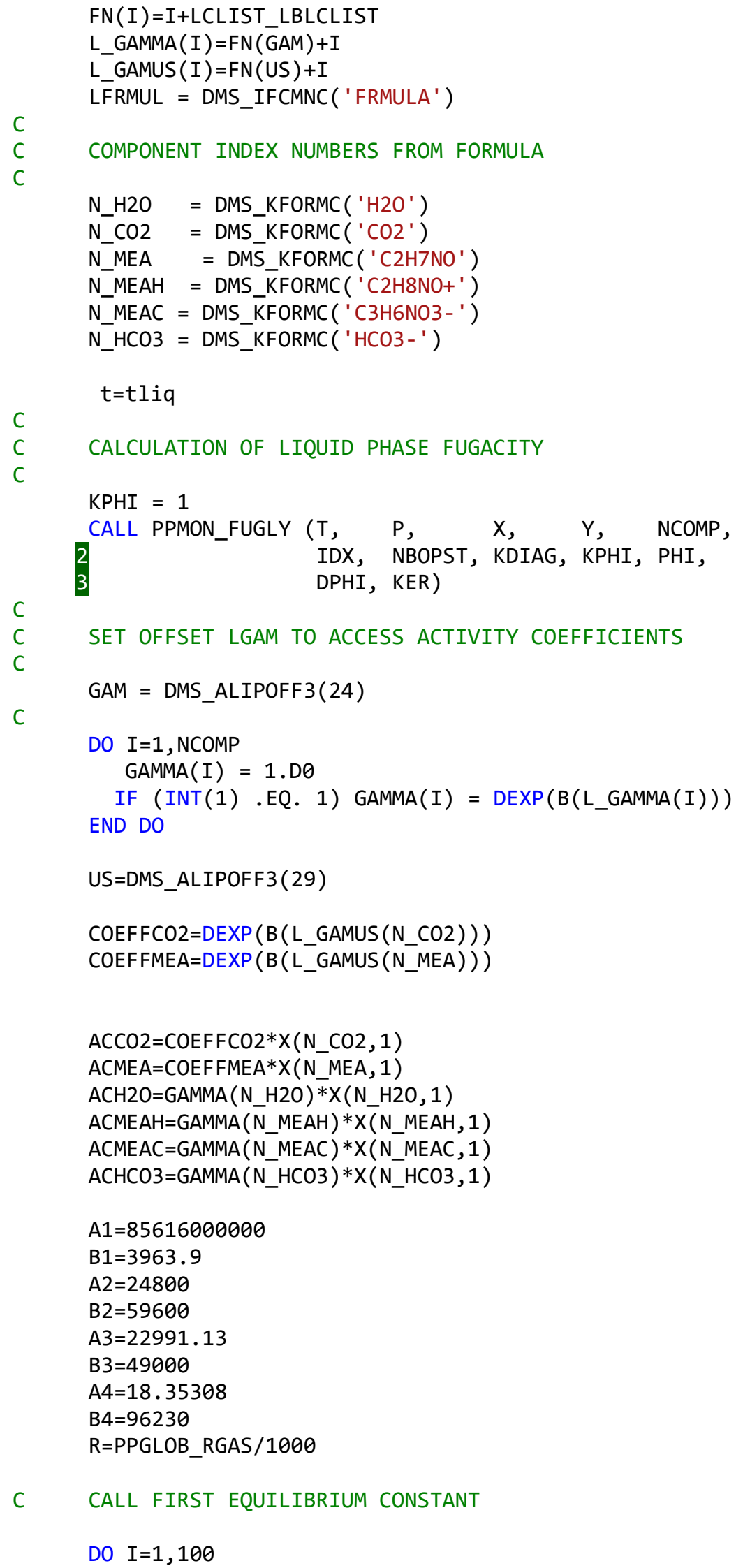


ENDDO

$\mathrm{STOI}(\mathrm{I})=0 \mathrm{DO}$

DO I=1, NCOMP

IF (IDX(I).EQ.N_MEA) STOI (I) $=-2 D \theta$

IF (IDX(I).EQ.N_CO2) STOI(I) $=-1 \mathrm{DO}$

IF (IDX(I).EQ.N_MEAH) STOI(I) $=1 \mathrm{DO}$

ENDDO

IF (IDX(I).EQ.N_MEAC) STOI(I) $=1 D \theta$

LNRKO=RGLOB_RMISS

CALL PPELC_ZKEQ $(T, 1,1,0$, STOI , 0 D 0 , NCOMP, IDX $, 0,1,1$, NBOPST, KDIAG, 2 LNRKO, P, IHELGK, DUM)

KEQ1 $=D E X P($ LNRKO $)$

C CALL SECOND EQUILIBRIUM CONSTANT

DO $I=1,100$

$\mathrm{STOI}(\mathrm{I})=\theta \mathrm{DO}$

ENDDO

DO $I=1$, NCOMP

IF (IDX(I).EQ.N MEA) STOI (I $)=-1 D \theta$

IF (IDX(I).EQ.N_CO2) STOI (I) $=-1 D 0$

IF (IDX(I).EQ.N_H2O) STOI(I) $=-1 D \theta$

IF (IDX(I).EQ.N_MEAH) STOI(I) $=1 D 0$

ENDDO

IF (IDX(I).EQ.N_HCO3) STOI(I) $=1 \mathrm{DO}$

LNRKO=RGLOB_RMISS

CALL PPELC_ZKEQ $(T, 1,1,0$, STOI , $, 0 D \theta, N C O M P, I D X, 0,1,1$, NBOPST , KDIAG, 2 LNRKO, P, IHELGK, DUM)

KEQ2 $=D E X P($ LNRKO $)$

C KINETIC MODEL (FORWARD/REVERSE REACTION RATES)

$\operatorname{RXNRATES}(1)=\operatorname{REAL}(1) * \operatorname{DEXP}(-\operatorname{REAL}(3) / \mathrm{R} *(1 / \operatorname{TLIQ}-1 / 298.15)) *$

2 (ACMEA**2*ACCO2-ACMEAC*ACMEAH/KEQ1)

$\operatorname{RXNRATES}(2)=\operatorname{REAL}(2) * \operatorname{DEXP}(-\operatorname{REAL}(4) / \mathrm{R} *(1 / \mathrm{TLIQ}-1 / 298.15)) *$

2(ACMEA*ACCO2-ACMEAH*ACHCO3/ (KEQ2*ACH2O))

DO $K=1, N R L(1)$

$\operatorname{RXNRATES}(K)=\operatorname{RXNRATES}(\mathrm{K}) *$ HLDLIQ

$\operatorname{ratel}(k)=$ rxnrates $(k)$

END DO

C

C

INITIALIZATION OF COMPONENT REACTION RATES

DO $I=1$, NCOMP

$\operatorname{RATES}(I)=0 . \mathrm{D} \theta$

END DO

C

COMPONENT REACTION RATES in $\mathrm{kmol} / \mathrm{sec}$ 


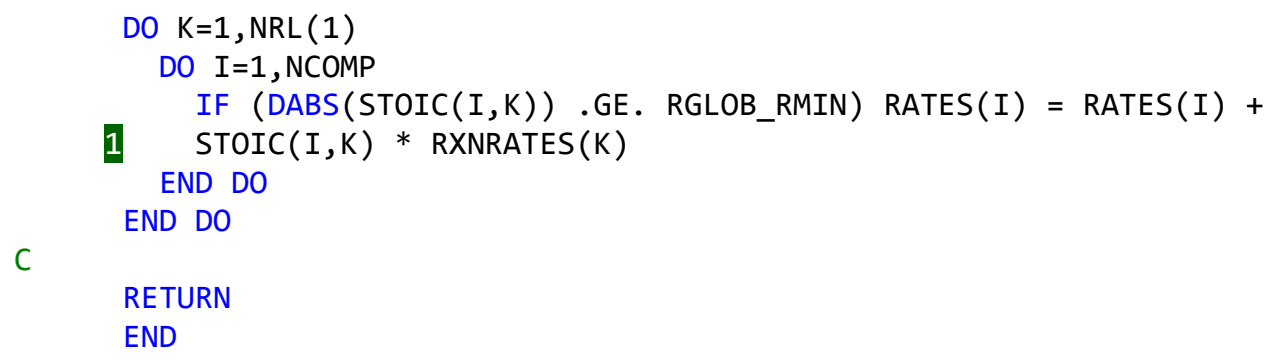




\section{Appendix D: Additional Thermodynamic Model Results}

In this appendix, the results of propagating the parametric uncertainty in the thermodynamic model through the process model are reported for all 23 cases of steady state data from NCCC.
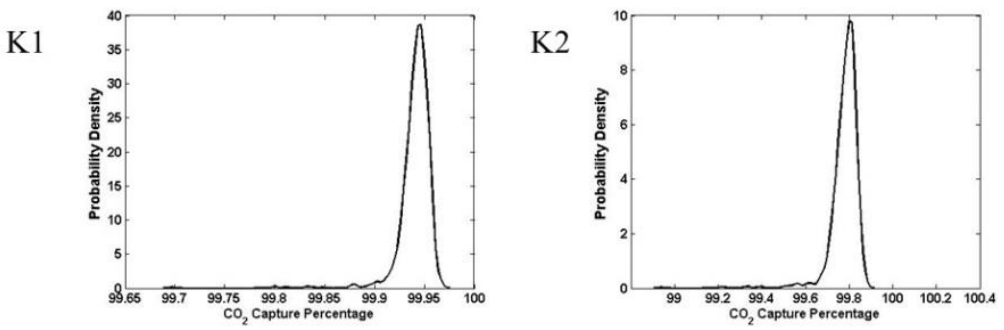

K3

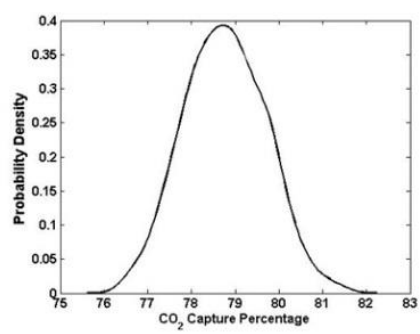

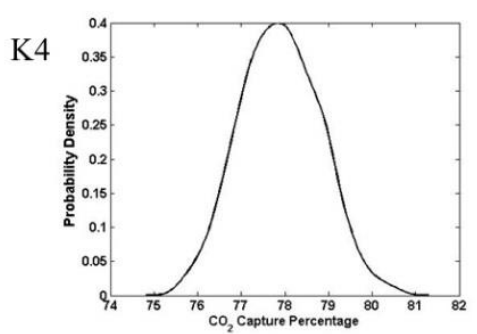

K5

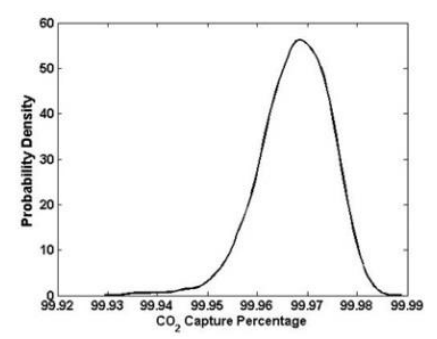

K7

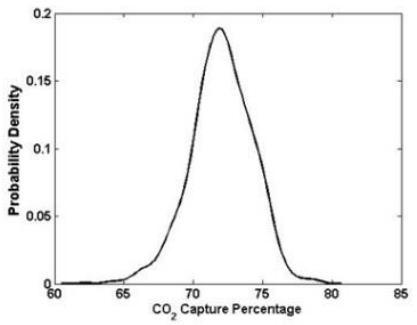

K10

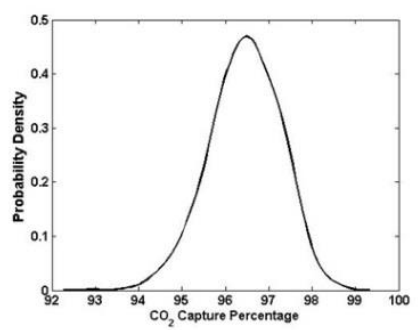

K8
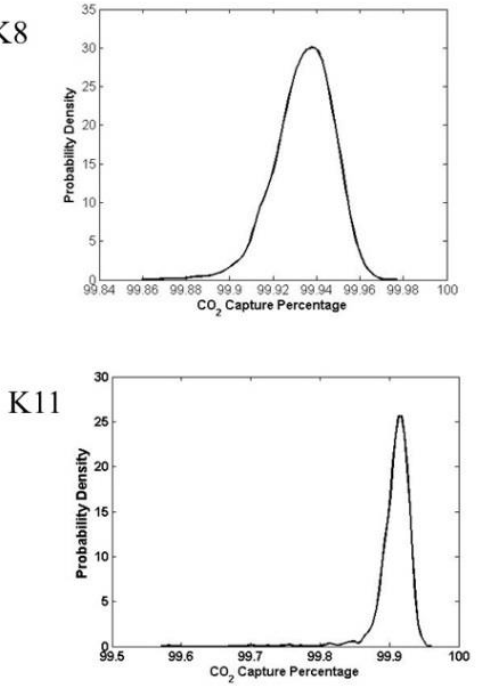

K6

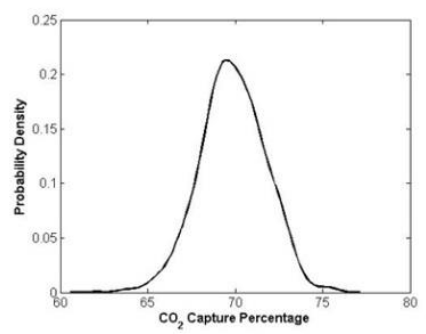

K9

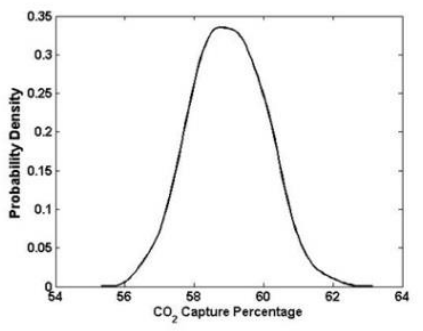

$\mathrm{K} 12$

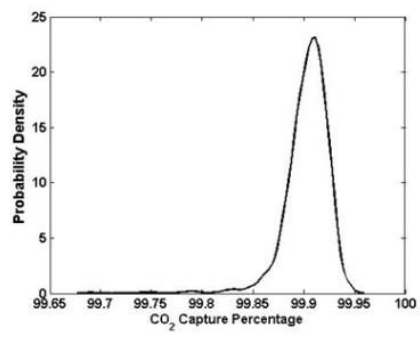



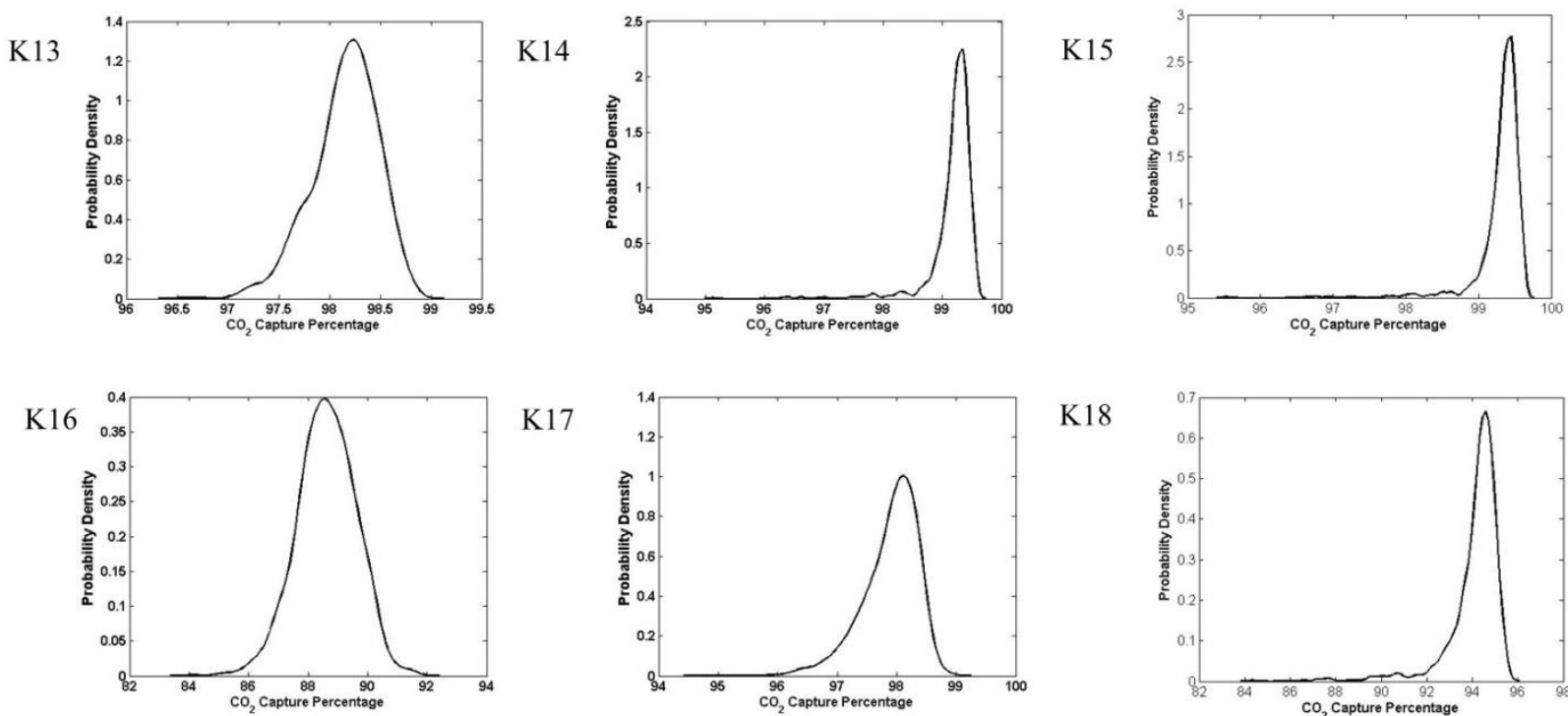

K17

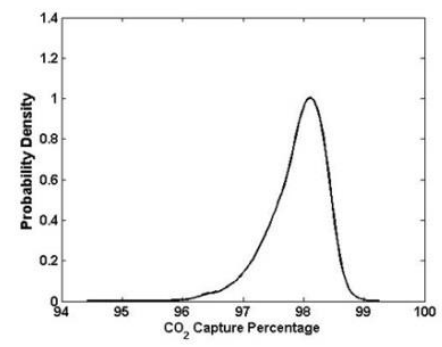

K18

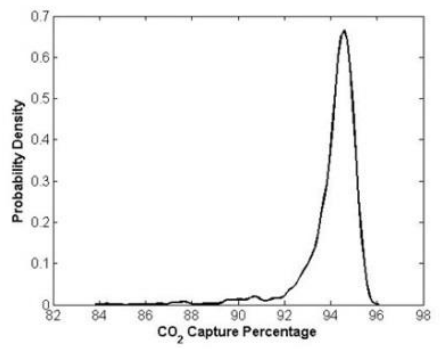

K19

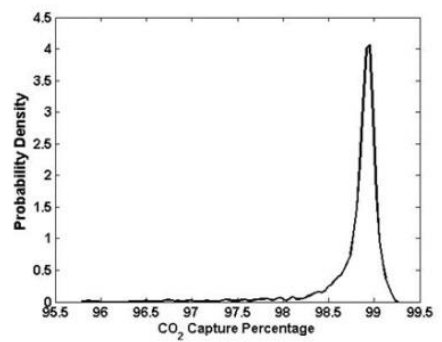

K20

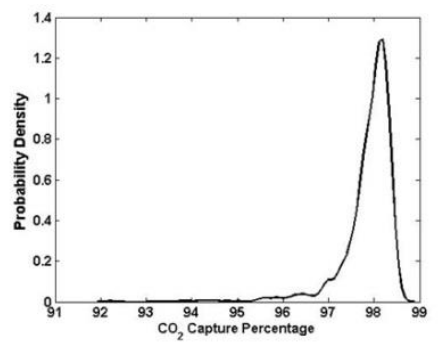

K21

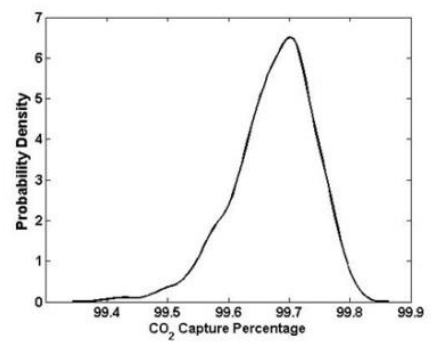

K22

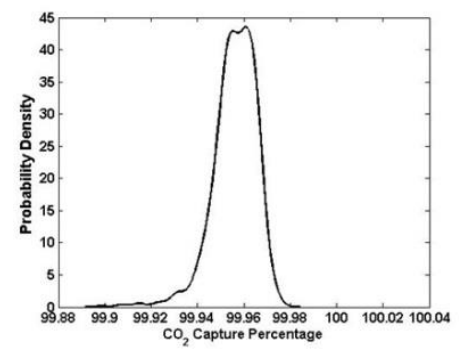

K23

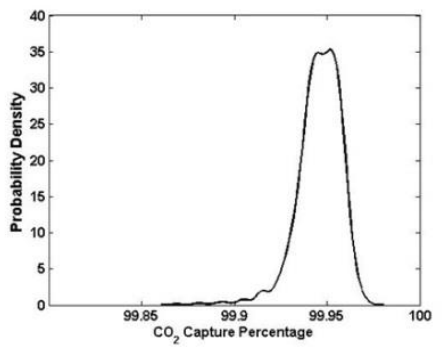

Figure D.1. Estimated probability density functions (pdfs) in $\mathrm{CO}_{2}$ capture percentage resulting from propagating thermodynamic model uncertainty through process model. 

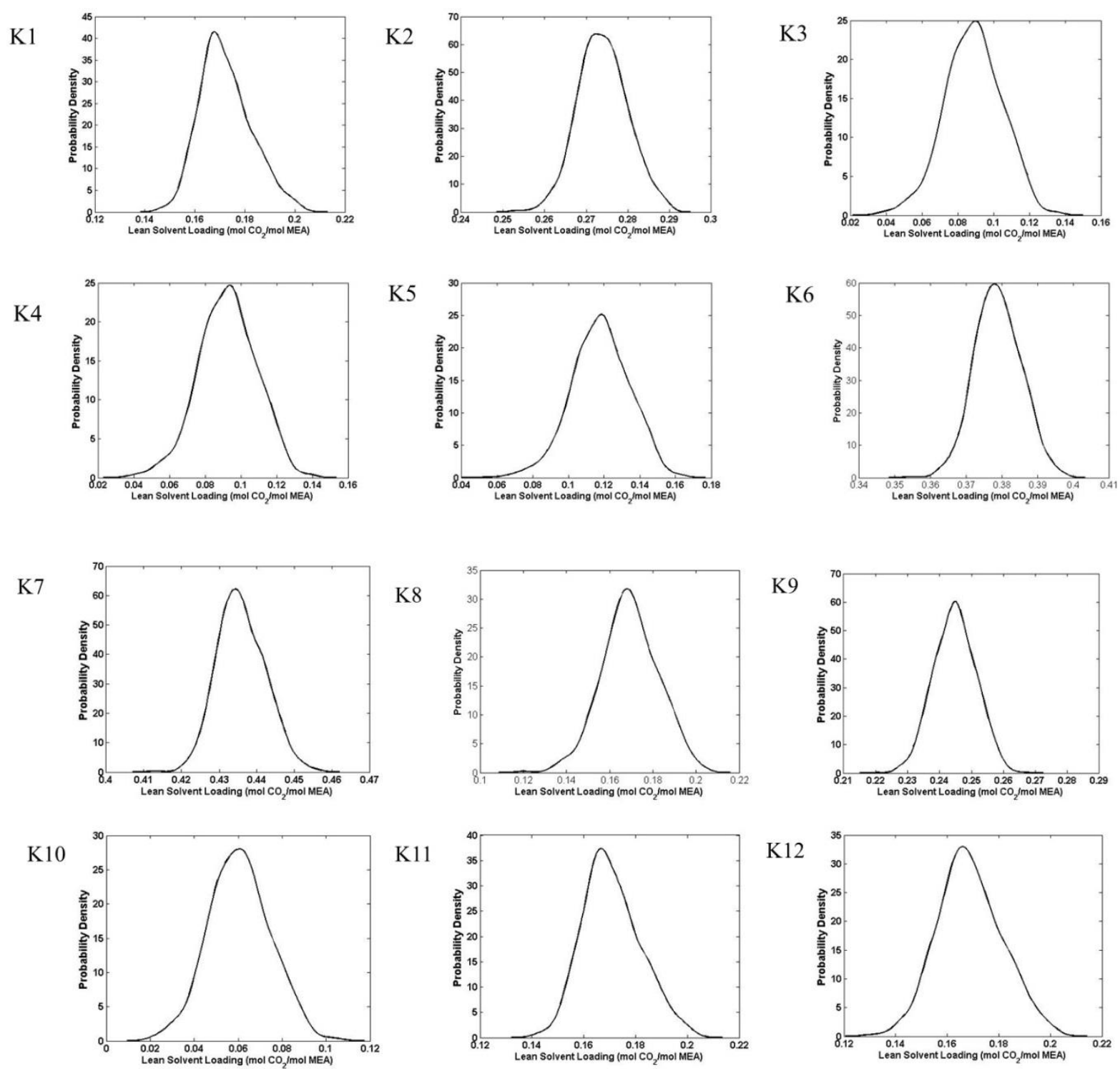

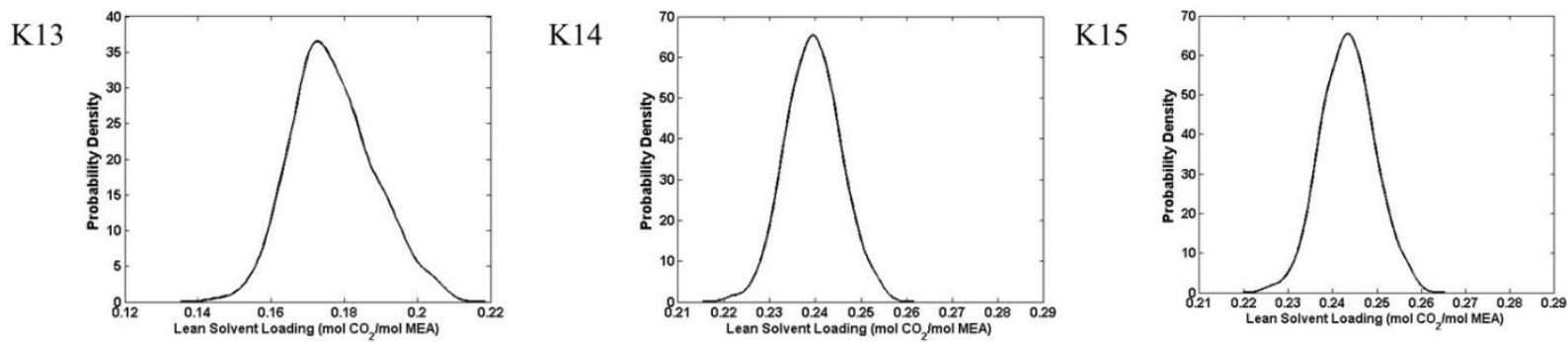

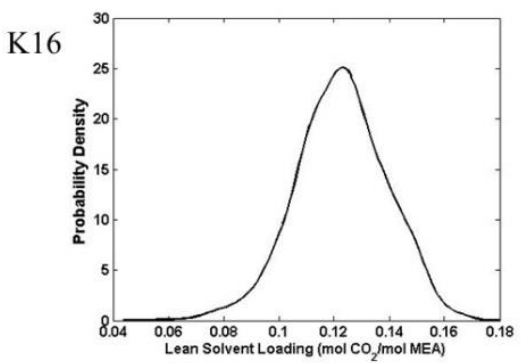

K17

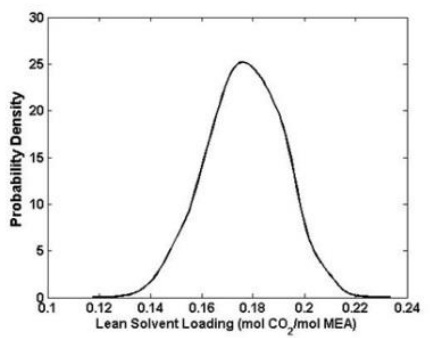

K18

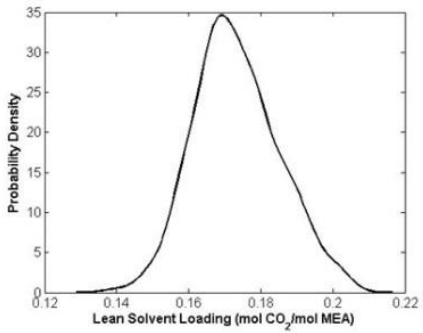

K19

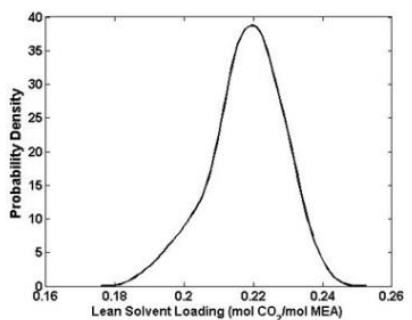

K22

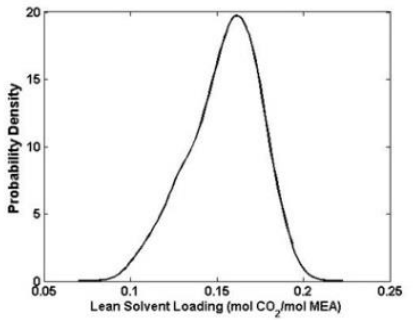

K20

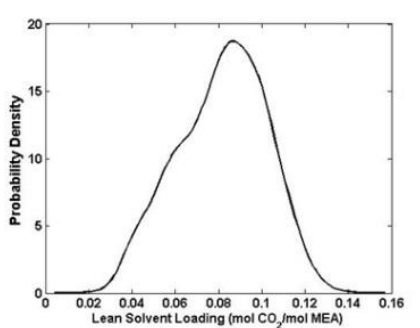

K23

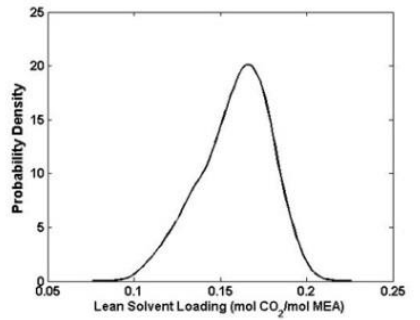

Figure D.2 Estimated probability density functions (pdfs) in lean solvent loading in stripper outlet resulting from propagating thermodynamic model uncertainty through process model. 
K1

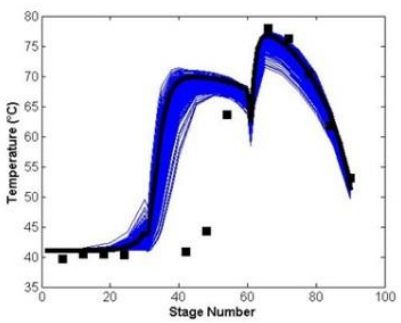

K4

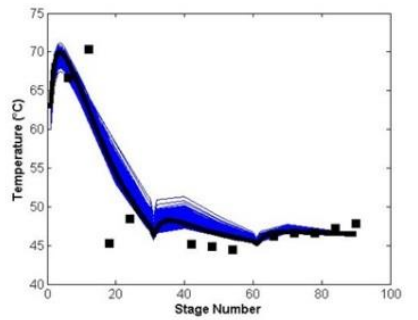

K7
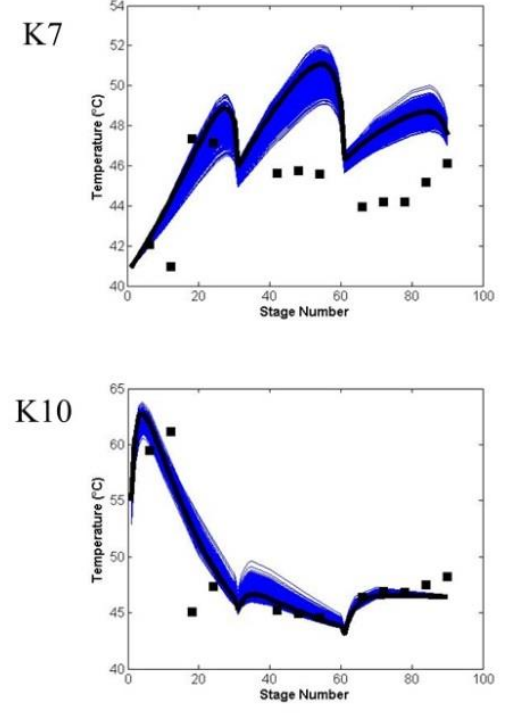

K2

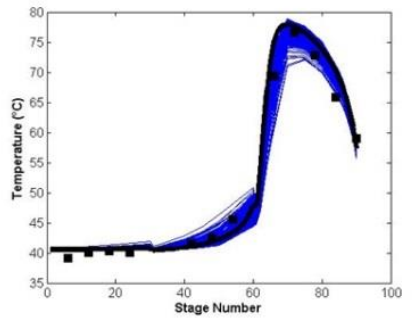

K5

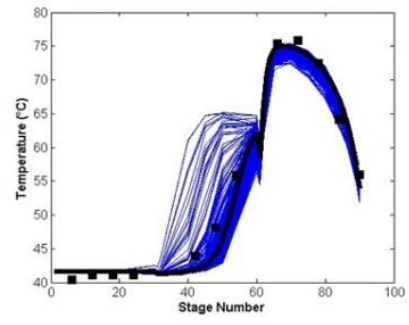

K8

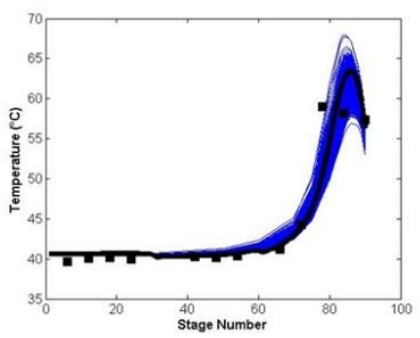

K11

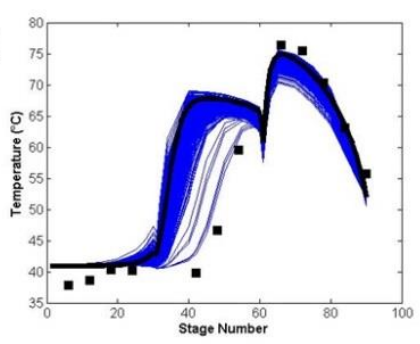

K3

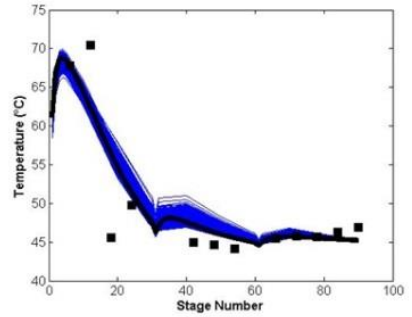

K6

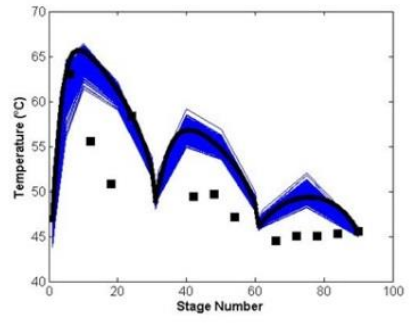

K9

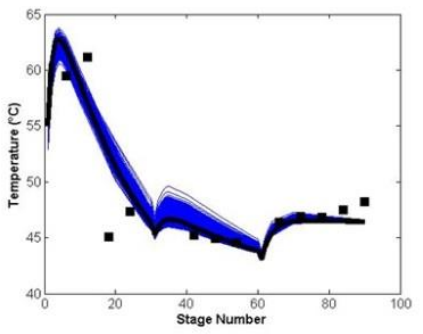

K12

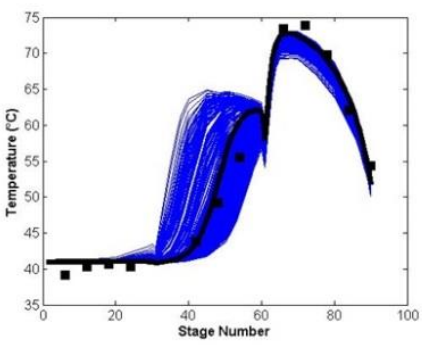


K13

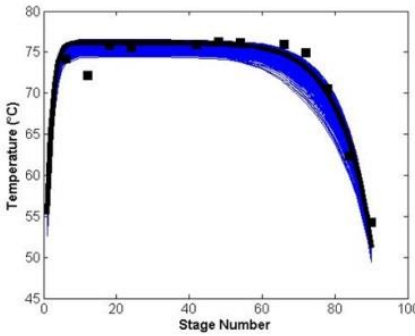

K16

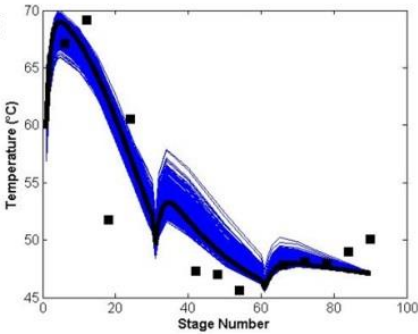

K19

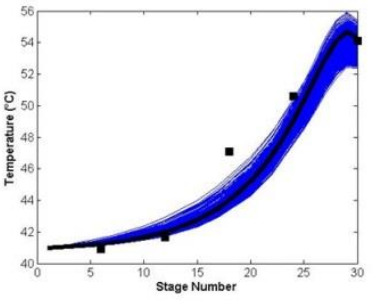

K22

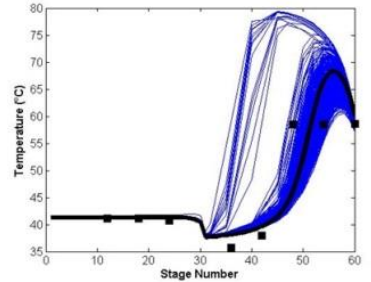

K14

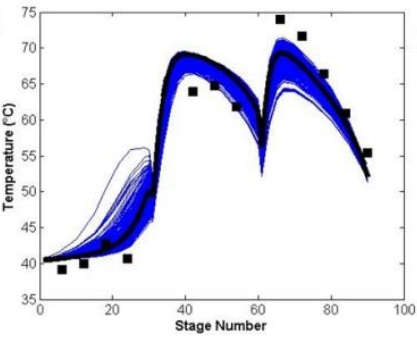

K17

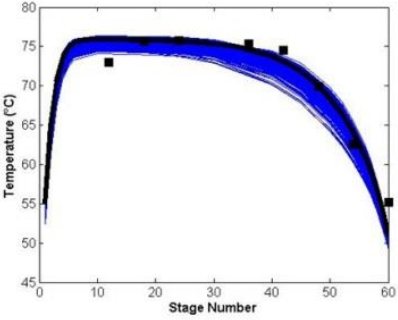

K20

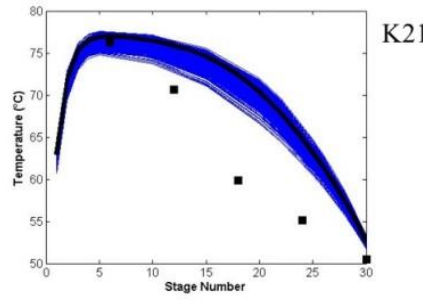

K23

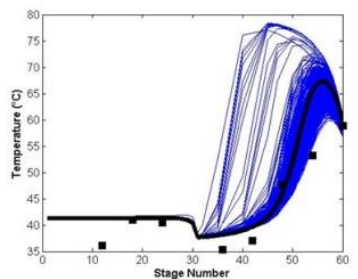

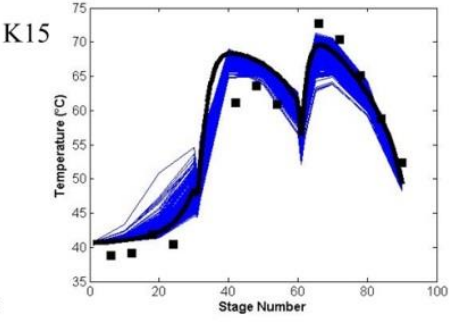
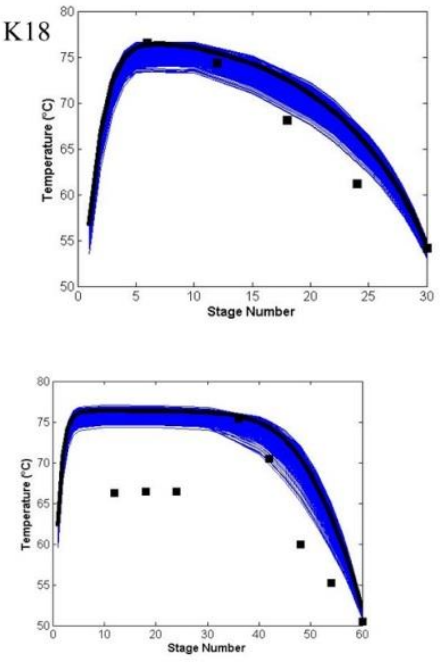

- Data

Stochastic Model

Figure D.3 Comparison of stochastic temperature profile to NCCC data for absorber column. Stages numbered from top of column to bottom. 
K1

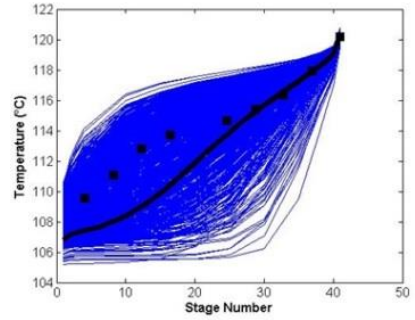

K4

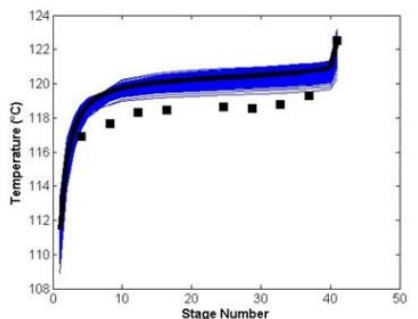

K7

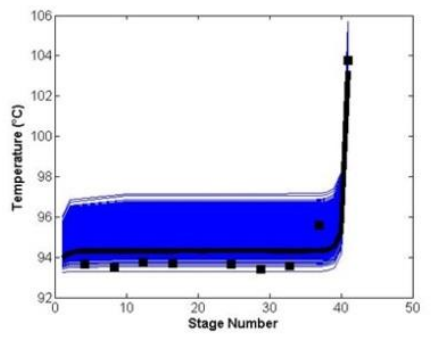

K10

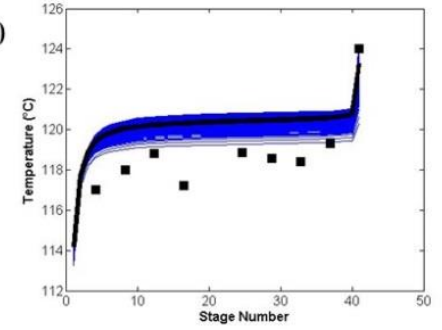

K2

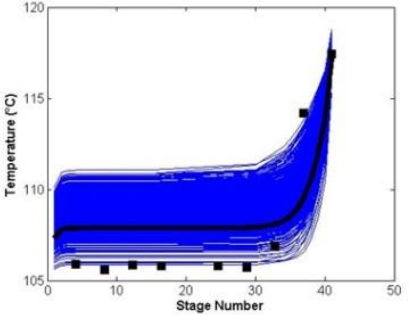

K5

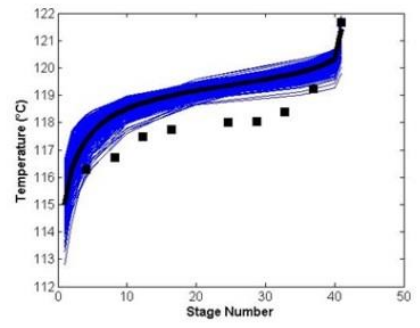

K8

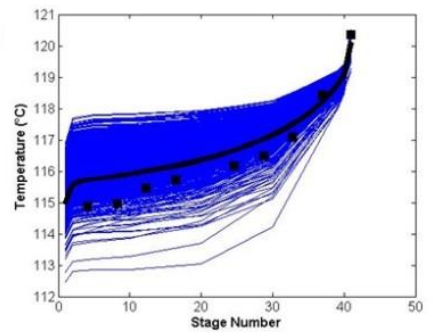

K11

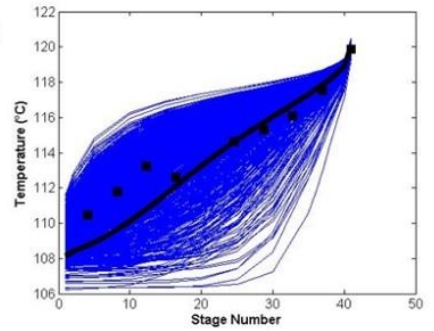

K3

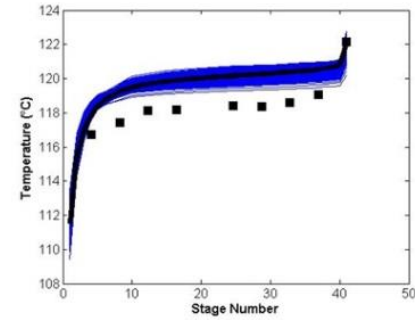

K6

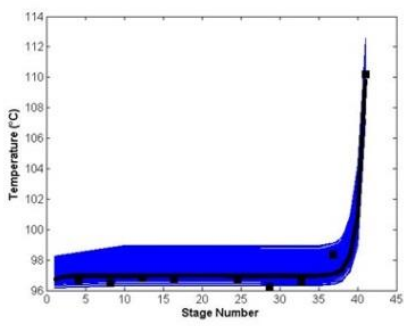

K9

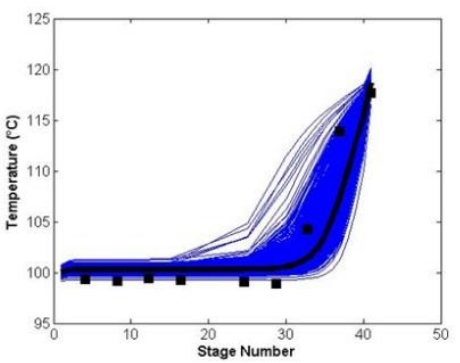

K12

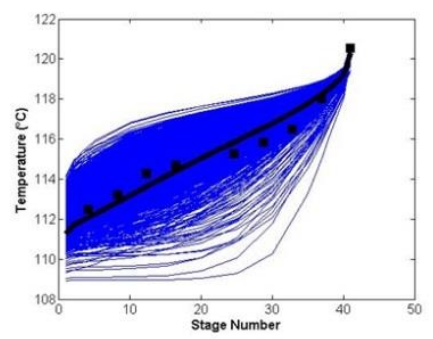


K13

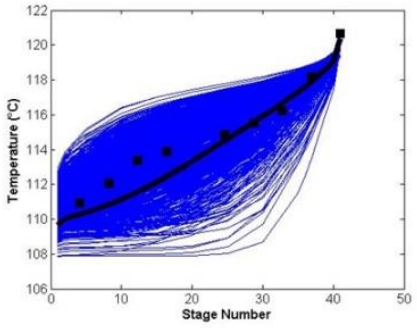

K16

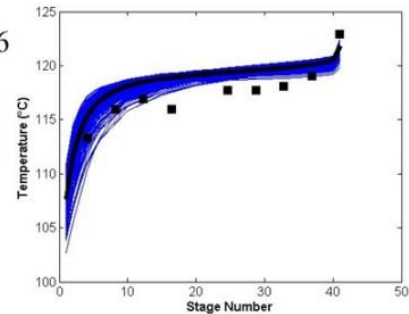

K19

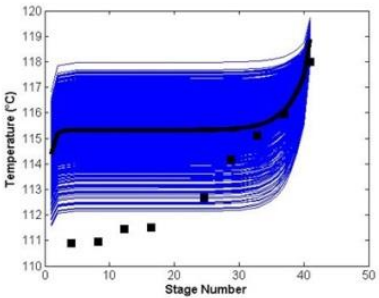

K22

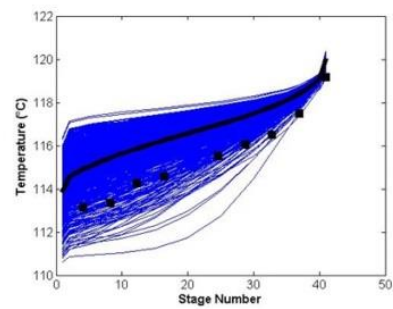

K14

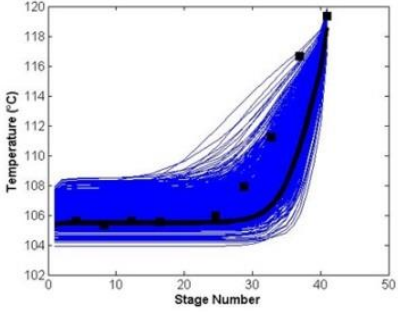

K17

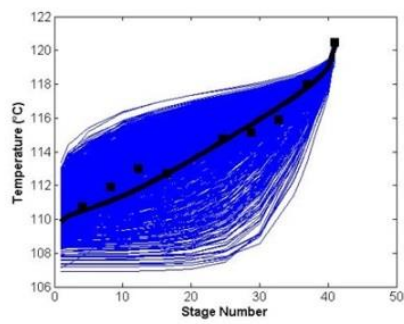

K20

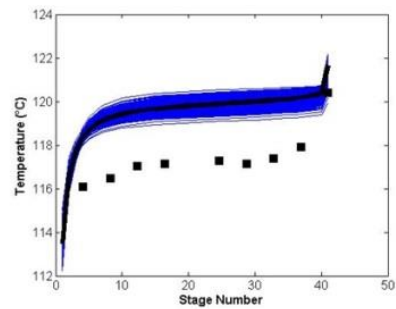

$\mathrm{K} 23$

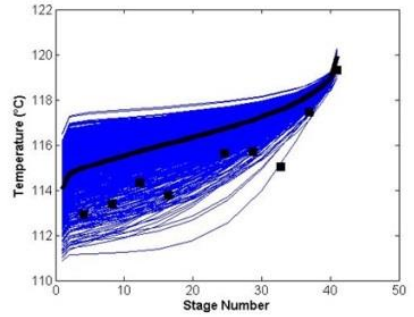

K15

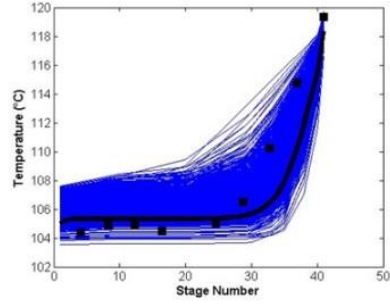

K18

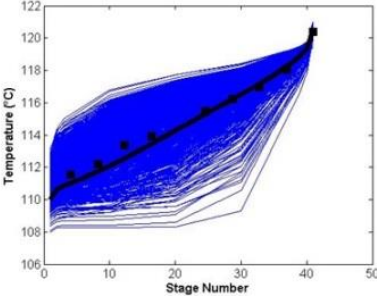

K21

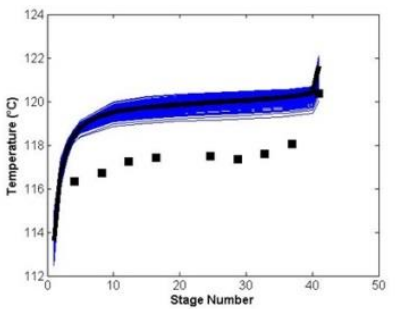

Deterministic Model Stochastic Model

Figure D.4 Comparison of stochastic temperature profile to NCCC data for stripper column. Stages numbered from top of column to bottom.

\section{Appendix E: Liquid Solvent Ranges for Alternative Process Configurations}

For all other process configurations other than three beds with intercooling, ranges of feasible operating lean solvent flowrate for a given flue gas flowrate and $\mathrm{CO}_{2}$ weight fraction and lean solvent loading are reported so that the absorber efficiency lies between $50-95 \% \mathrm{CO}_{2}$ capture. An additional constraint is that the lean solvent flowrate also lies between $3000-13000 \mathrm{~kg} / \mathrm{hr}$, 
based on the ranges given in the original NCCC test. The ranges of lean solvent flowrate for which these criteria are met are given in Tables E.1-, respectively, for the following alternate process configurations: three beds without intercooling, two beds without intercooling, two beds with intercooling, and one bed. 
Table E.1. Ranges of liquid flowrate recommended for absorber column with three beds without intercooling

\begin{tabular}{|c|c|c|c|c|}
\hline \multirow{2}{*}{$\begin{array}{c}\text { Flue Gas } \mathrm{CO}_{2} \\
\text { Weight Fraction }\end{array}$} & \multirow{2}{*}{$\begin{array}{c}\text { Flue Gas } \\
\text { Flowrate }(\mathrm{kg} / \mathrm{hr})\end{array}$} & \multirow{2}{*}{$\begin{array}{c}\text { Lean Solvent } \\
\text { Loading } \\
\left(\mathrm{mol} \mathrm{CO}_{2} / \mathrm{MEA}\right)\end{array}$} & \multicolumn{2}{|c|}{ Lean Solvent Flowrate $(\mathrm{kg} / \mathrm{hr})$} \\
\hline & & & Minimum & Maximum \\
\hline 0.125 & 1000 & 0.1 & NA & NA \\
\hline 0.125 & 1000 & 0.2 & NA & NA \\
\hline 0.125 & 1000 & 0.25 & NA & NA \\
\hline 0.125 & 1000 & 0.3 & 3000 & 5180 \\
\hline 0.150 & 1000 & 0.1 & NA & NA \\
\hline 0.150 & 1000 & 0.2 & NA & NA \\
\hline 0.150 & 1000 & 0.25 & 3000 & 3790 \\
\hline 0.150 & 1000 & 0.3 & 3000 & 5650 \\
\hline 0.175 & 1000 & 0.1 & NA & NA \\
\hline 0.175 & 1000 & 0.2 & NA & NA \\
\hline 0.175 & 1000 & 0.25 & 3000 & 4780 \\
\hline 0.175 & 1000 & 0.3 & 3000 & 6180 \\
\hline 0.125 & 2250 & 0.1 & 3000 & 3130 \\
\hline 0.125 & 2250 & 0.2 & 3000 & 4410 \\
\hline 0.125 & 2250 & 0.25 & 3000 & 11150 \\
\hline 0.125 & 2250 & 0.3 & 3310 & 12330 \\
\hline 0.150 & 2250 & 0.1 & 3000 & 3730 \\
\hline 0.150 & 2250 & 0.2 & 3000 & 5270 \\
\hline 0.150 & 2250 & 0.25 & 3120 & 12360 \\
\hline 0.150 & 2250 & 0.3 & 3920 & 13000 \\
\hline 0.175 & 2250 & 0.1 & 3000 & 4330 \\
\hline 0.175 & 2250 & 0.2 & 3000 & 6140 \\
\hline 0.175 & 2250 & 0.25 & 3600 & 13000 \\
\hline 0.175 & 2250 & 0.3 & 4550 & 13000 \\
\hline 0.125 & 3000 & 0.1 & 3000 & 4250 \\
\hline 0.125 & 3000 & 0.2 & 3000 & 6110 \\
\hline 0.125 & 3000 & 0.25 & 3540 & 13000 \\
\hline 0.125 & 3000 & 0.3 & 4480 & 13000 \\
\hline 0.150 & 3000 & 0.1 & 3000 & 5060 \\
\hline 0.150 & 3000 & 0.2 & 3470 & 7380 \\
\hline 0.150 & 3000 & 0.25 & 4190 & 13000 \\
\hline 0.150 & 3000 & 0.3 & 5310 & 13000 \\
\hline 0.175 & 3000 & 0.1 & 3000 & 5860 \\
\hline 0.175 & 3000 & 0.2 & 4000 & 8750 \\
\hline 0.175 & 3000 & 0.25 & 4840 & 13000 \\
\hline 0.175 & 3000 & 0.3 & 6170 & 13000 \\
\hline
\end{tabular}


Table E.2. Ranges of liquid flowrate recommended for absorber column with two beds without intercooling

\begin{tabular}{|c|c|c|c|c|}
\hline \multirow{2}{*}{$\begin{array}{c}\text { Flue Gas } \mathrm{CO}_{2} \\
\text { Weight Fraction }\end{array}$} & \multirow{2}{*}{$\begin{array}{c}\text { Flue Gas } \\
\text { Flowrate }(\mathrm{kg} / \mathrm{hr})\end{array}$} & \multirow{2}{*}{$\begin{array}{c}\text { Lean Solvent } \\
\text { Loading } \\
\left(\mathrm{mol} \mathrm{CO}_{2} / \mathrm{MEA}\right)\end{array}$} & \multicolumn{2}{|c|}{ Lean Solvent Flowrate (kg/hr) } \\
\hline & & & Minimum & Maximum \\
\hline 0.125 & 1000 & 0.1 & NA & NA \\
\hline 0.125 & 1000 & 0.2 & NA & NA \\
\hline 0.125 & 1000 & 0.25 & NA & NA \\
\hline 0.125 & 1000 & 0.3 & 3000 & 5180 \\
\hline 0.150 & 1000 & 0.1 & NA & NA \\
\hline 0.150 & 1000 & 0.2 & NA & NA \\
\hline 0.150 & 1000 & 0.25 & 3000 & 3870 \\
\hline 0.150 & 1000 & 0.3 & 3000 & 5690 \\
\hline 0.175 & 1000 & 0.1 & NA & NA \\
\hline 0.175 & 1000 & 0.2 & NA & NA \\
\hline 0.175 & 1000 & 0.25 & 3000 & 4810 \\
\hline 0.175 & 1000 & 0.3 & 3000 & 6180 \\
\hline 0.125 & 2250 & 0.1 & 3000 & 3310 \\
\hline 0.125 & 2250 & 0.2 & 3000 & 5100 \\
\hline 0.125 & 2250 & 0.25 & 3000 & 11290 \\
\hline 0.125 & 2250 & 0.3 & 3460 & 12330 \\
\hline 0.150 & 2250 & 0.1 & 3000 & 3910 \\
\hline 0.150 & 2250 & 0.2 & 3000 & 6000 \\
\hline 0.150 & 2250 & 0.25 & 3200 & 12440 \\
\hline 0.150 & 2250 & 0.3 & 4090 & 13000 \\
\hline 0.175 & 2250 & 0.1 & 3000 & 4530 \\
\hline 0.175 & 2250 & 0.2 & 3050 & 6980 \\
\hline 0.175 & 2250 & 0.25 & 3710 & 13000 \\
\hline 0.175 & 2250 & 0.3 & 4750 & 13000 \\
\hline 0.125 & 3000 & 0.1 & 3000 & 4650 \\
\hline 0.125 & 3000 & 0.2 & 3010 & 8070 \\
\hline 0.125 & 3000 & 0.25 & 3680 & 13000 \\
\hline 0.125 & 3000 & 0.3 & 4740 & 13000 \\
\hline 0.150 & 3000 & 0.1 & 3000 & 5460 \\
\hline 0.150 & 3000 & 0.2 & 3570 & 9460 \\
\hline 0.150 & 3000 & 0.25 & 4350 & 13000 \\
\hline 0.150 & 3000 & 0.3 & 5600 & 13000 \\
\hline 0.175 & 3000 & 0.1 & 3030 & 6310 \\
\hline 0.175 & 3000 & 0.2 & 4120 & 10960 \\
\hline 0.175 & 3000 & 0.25 & 5020 & 13000 \\
\hline 0.175 & 3000 & 0.3 & 6470 & 13000 \\
\hline
\end{tabular}


Table E.3. Ranges of liquid flowrate recommended for absorber column with two beds with intercooling

\begin{tabular}{|c|c|c|c|c|}
\hline \multirow{2}{*}{$\begin{array}{c}\text { Flue Gas } \mathrm{CO}_{2} \\
\text { Weight Fraction }\end{array}$} & \multirow{2}{*}{$\begin{array}{c}\text { Flue Gas } \\
\text { Flowrate }(\mathrm{kg} / \mathrm{hr})\end{array}$} & \multirow{2}{*}{$\begin{array}{c}\text { Lean Solvent } \\
\text { Loading } \\
\left.\text { (mol CO } \mathrm{CO}_{2} / \mathrm{MEA}\right)\end{array}$} & \multicolumn{2}{|c|}{ Lean Solvent Flowrate $(\mathrm{kg} / \mathrm{hr})$} \\
\hline & & & Minimum & Maximum \\
\hline 0.125 & 1000 & 0.1 & NA & NA \\
\hline 0.125 & 1000 & 0.2 & NA & NA \\
\hline 0.125 & 1000 & 0.25 & NA & NA \\
\hline 0.125 & 1000 & 0.3 & 3000 & 3480 \\
\hline 0.150 & 1000 & 0.1 & NA & NA \\
\hline 0.150 & 1000 & 0.2 & NA & NA \\
\hline 0.150 & 1000 & 0.25 & NA & NA \\
\hline 0.150 & 1000 & 0.3 & 3000 & 4082 \\
\hline 0.175 & 1000 & 0.1 & NA & NA \\
\hline 0.175 & 1000 & 0.2 & NA & NA \\
\hline 0.175 & 1000 & 0.25 & 3000 & 3380 \\
\hline 0.175 & 1000 & 0.3 & 3000 & 4620 \\
\hline 0.125 & 2250 & 0.1 & 3000 & 3310 \\
\hline 0.125 & 2250 & 0.2 & 3000 & 4940 \\
\hline 0.125 & 2250 & 0.25 & 3000 & 6910 \\
\hline 0.125 & 2250 & 0.3 & 3450 & 11000 \\
\hline 0.150 & 2250 & 0.1 & 3000 & 3910 \\
\hline 0.150 & 2250 & 0.2 & 3000 & 5770 \\
\hline 0.150 & 2250 & 0.25 & 3200 & 7960 \\
\hline 0.150 & 2250 & 0.3 & 4070 & 12080 \\
\hline 0.175 & 2250 & 0.1 & 3000 & 4520 \\
\hline 0.175 & 2250 & 0.2 & 3050 & 6630 \\
\hline 0.175 & 2250 & 0.25 & 3690 & 9010 \\
\hline 0.175 & 2250 & 0.3 & 4700 & 13000 \\
\hline 0.125 & 3000 & 0.1 & 3000 & 4650 \\
\hline 0.125 & 3000 & 0.2 & 3010 & 7460 \\
\hline 0.125 & 3000 & 0.25 & 3670 & 11460 \\
\hline 0.125 & 3000 & 0.3 & 4730 & 13000 \\
\hline 0.150 & 3000 & 0.1 & 3000 & 5460 \\
\hline 0.150 & 3000 & 0.2 & 3570 & 8550 \\
\hline 0.150 & 3000 & 0.25 & 4350 & 12640 \\
\hline 0.150 & 3000 & 0.3 & 5580 & 13000 \\
\hline 0.175 & 3000 & 0.1 & 3030 & 6300 \\
\hline 0.175 & 3000 & 0.2 & 4120 & 9690 \\
\hline 0.175 & 3000 & 0.25 & 5020 & 13000 \\
\hline 0.175 & 3000 & 0.3 & 6430 & 13000 \\
\hline
\end{tabular}


Table E.4. Ranges of liquid flowrate recommended for absorber column with one bed

\begin{tabular}{|c|c|c|c|c|}
\hline \multirow{2}{*}{$\begin{array}{c}\text { Flue Gas } \mathrm{CO}_{2} \\
\text { Weight Fraction }\end{array}$} & \multirow{2}{*}{$\begin{array}{c}\text { Flue Gas } \\
\text { Flowrate }(\mathrm{kg} / \mathrm{hr})\end{array}$} & \multirow{2}{*}{$\begin{array}{c}\text { Lean Solvent } \\
\text { Loading } \\
(\mathrm{mol} \mathrm{CO} / \mathrm{MEA})\end{array}$} & \multicolumn{2}{|c|}{ Lean Solvent Flowrate $(\mathrm{kg} / \mathrm{hr})$} \\
\hline & & & Minimum & Maximum \\
\hline 0.125 & 1000 & 0.1 & NA & NA \\
\hline 0.125 & 1000 & 0.2 & NA & NA \\
\hline 0.125 & 1000 & 0.25 & 3000 & 4260 \\
\hline 0.125 & 1000 & 0.3 & 3000 & 5810 \\
\hline 0.150 & 1000 & 0.1 & NA & NA \\
\hline 0.150 & 1000 & 0.2 & NA & NA \\
\hline 0.150 & 1000 & 0.25 & 3000 & 4990 \\
\hline 0.150 & 1000 & 0.3 & 3000 & 6320 \\
\hline 0.175 & 1000 & 0.1 & NA & NA \\
\hline 0.175 & 1000 & 0.2 & 3000 & 3640 \\
\hline 0.175 & 1000 & 0.25 & 3000 & 5490 \\
\hline 0.175 & 1000 & 0.3 & 3000 & 6890 \\
\hline 0.125 & 2250 & 0.1 & 3000 & 5390 \\
\hline 0.125 & 2250 & 0.2 & 3000 & 13000 \\
\hline 0.125 & 2250 & 0.25 & 3060 & 13000 \\
\hline 0.125 & 2250 & 0.3 & 4130 & 13000 \\
\hline 0.150 & 2250 & 0.1 & 3000 & 5940 \\
\hline 0.150 & 2250 & 0.2 & 3000 & 13000 \\
\hline 0.150 & 2250 & 0.25 & 3620 & 13000 \\
\hline 0.150 & 2250 & 0.3 & 4840 & 13000 \\
\hline 0.175 & 2250 & 0.1 & 3000 & 6470 \\
\hline 0.175 & 2250 & 0.2 & 3340 & 13000 \\
\hline 0.175 & 2250 & 0.25 & 4150 & 13000 \\
\hline 0.175 & 2250 & 0.3 & 5560 & 13000 \\
\hline 0.125 & 3000 & 0.1 & 3000 & 10510 \\
\hline 0.125 & 3000 & 0.2 & 3440 & 13000 \\
\hline 0.125 & 3000 & 0.25 & 4390 & 13000 \\
\hline 0.125 & 3000 & 0.3 & 6070 & 13000 \\
\hline 0.150 & 3000 & 0.1 & 3000 & 10980 \\
\hline 0.150 & 3000 & 0.2 & 4030 & 13000 \\
\hline 0.150 & 3000 & 0.25 & 5120 & 13000 \\
\hline 0.150 & 3000 & 0.3 & 7120 & 13000 \\
\hline 0.175 & 3000 & 0.1 & 3270 & 11680 \\
\hline 0.175 & 3000 & 0.2 & 4630 & 13000 \\
\hline 0.175 & 3000 & 0.25 & 5840 & 13000 \\
\hline 0.175 & 3000 & 0.3 & 8170 & 13000 \\
\hline
\end{tabular}




\section{Appendix F. Scale-Up Uncertainty Quantification}

The purpose of this section is to propose a methodology for scale-up uncertainty quantification, in which the effect of the absorber column radial size on its performance is analyzed. Since columns are represented as one-dimensional (axial only) in Aspen Plus ${ }^{\circledR}$ and practically all leading commercial process simulation software mainly because of computational tractability, the effect of liquid and gas maldistribution on $\mathrm{CO}_{2}$ capture percentage cannot be considered. Although this work is ongoing and will require new experimental data for proper implementation, some preliminary methodology and results are presented here.

In order to analyze radial variation in the column, a hypothetical column is considered in the schematic shown in Figure F.1.

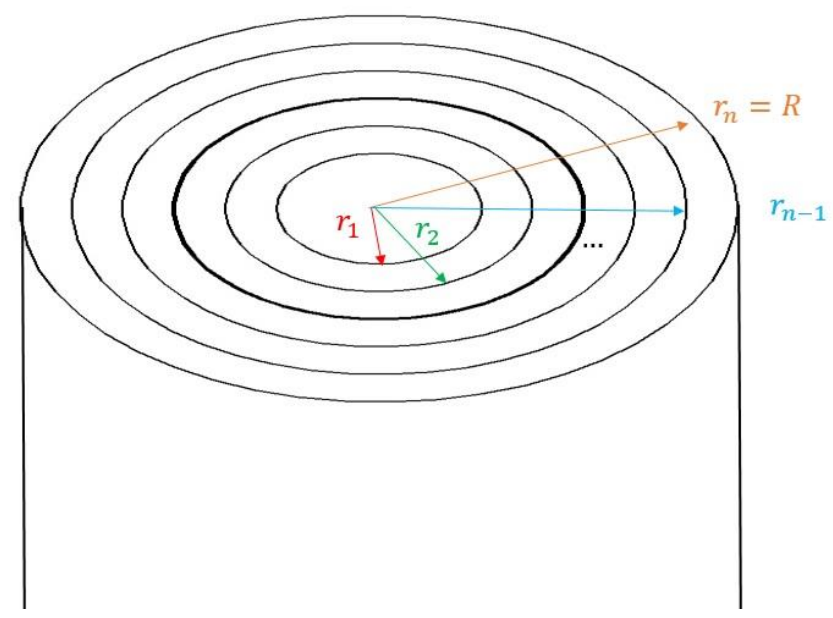

Figure F.1. Schematic of absorber column with radial variation taken into consideration

The column is discretized into $n$ radial sections. For each section, fractions of the total amount of liquid $(L)$ and gas $(G)$ flow rates are apportioned, and these fractions are denoted as $\varphi_{L i}$ and $\varphi_{G i}$. Previous work (Mohamed Ali et al., 2003) has shown that gas maldistribution has small effect on column efficiency, so the gas distribution may be modeled as uniform in this work. The gas fraction for each column section $i$ will be proportional to the fraction of the column area occupied by the section, and calculated as:

$$
\varphi_{G i}=\frac{r_{i}^{2}-r_{i-1}^{2}}{R^{2}}, \forall i \in(1, n)
$$

where $r_{0}=0$ by definition. The liquid distribution fraction may be represented generically as: 


$$
\begin{gathered}
\varphi_{L i}=\frac{\int_{r_{i-1}}^{r_{i}} r \varphi_{L}(r) d r}{R^{2}}, \forall i \in(1, n) \\
\int_{0}^{R} r \varphi_{L}(r) d r=R^{2}
\end{gathered}
$$

For a one-dimensional column simulation in Aspen Plus ${ }^{\circledR}$, the $\mathrm{CO}_{2}$ capture percentage is essentially a function of the liquid to gas ratio entering the column $(\delta)$ and the inlet lean loading $\left(\alpha_{\text {lean }}\right)$, provided that other variables, including column temperature, pressure, and gas composition are at constant values. If each discretized section of the column is modeled as an individual column, the percentage of capture from that section may be denoted as:

$$
\begin{gathered}
C A P_{i}=f\left(\delta_{i}, \alpha_{\text {lean }}\right) \\
\delta_{i}=\frac{\varphi_{L i} L}{\varphi_{G i} G} \triangleq \frac{L_{i}}{G_{i}}
\end{gathered}
$$

The mass of $\mathrm{CO}_{2}$ remaining from a section $i$ is calculated as:

$$
C O_{2, \text { out }, i}=w_{\mathrm{CO}_{2}} G_{i}\left(1-\frac{C A P_{i}}{100}\right)
$$

where $w_{\mathrm{CO}_{2}}$ is the weight fraction of $\mathrm{CO}_{2}$ in the gas entering the column, a constant over all discretizations. The overall $\mathrm{CO}_{2}$ capture percentage for the column is calculated as:

$$
C A P_{\text {tot }}=100 \% *\left(1-\frac{\sum_{i=1}^{n} C O_{2, o u t, i}}{w_{\mathrm{CO}_{2}} G}\right)
$$

Theoretically, the value of the overall $\mathrm{CO}_{2}$ capture percentage calculated here should be lower than that calculated from the one-dimensional column model if there is liquid maldistribution.

It is very difficult to develop an accurate liquid distribution model since it depends on specifics of the packing hardware including its geometry and dimensions, property of the liquid, liquid distributors and sprays (if present), wall effects, local characteristics such as bubbles, jets, dry spots, and so on that strongly depend on surface characteristics and wetting properties of the packing. These effects are difficult to model especially in a process simulation software environment. One plausible form of the liquid distribution model can be:

$$
\varphi_{L}=\varphi_{L}(r \mid \tilde{\theta})
$$

where $\tilde{\theta}$ represents a set of parameters that can be fit to experimental data. A sample liquid distribution function is taken to be the radial basis function, given by: 


$$
\varphi_{L}(r \mid \tilde{\theta})=\beta \exp \left(-\theta r^{2}\right)
$$

where $\beta$ is a constant that must be calculated to satisfy the integral condition in Eq. F.3.

Based on the shapes of the distribution profiles presented in Nawrocki et al. (1991), which makes use of a mechanistic model to estimate liquid distribution in packing, this could be a reasonable choice for a starting point for the liquid distribution model.

A case study is performed in which the radial basis function (with $\theta=1$ ) as given in Eq. F.9 is used to represent the liquid distribution. Some specifications for this analysis are presented in Table F.1.

Table F.1. Operating specifications for scale-up UQ case study

\begin{tabular}{cc}
\hline Specifications & Value \\
\hline $\mathrm{G}(\mathrm{kg} / \mathrm{hr})$ & 2260 \\
$\alpha_{\text {lean }}(\mathrm{mol} \mathrm{CO} / \mathrm{mol} \mathrm{MEA})$ & 0.3 \\
$w_{\mathrm{CO}_{2}}$ & 0.15958 \\
\hline
\end{tabular}

A sensitivity study is performed to determine the effect of the number of column discretizations on the prediction of $\mathrm{CO}_{2}$ capture. For this analysis, a default radius value of $R=1$, noting that $R$ is a dimensionless relative radius variable here. A surrogate model is used to calculate the $\mathrm{CO}_{2}$ capture percentage, based on the relationship given in Eq. F.4. The results of this study are given in Figure F.2. 


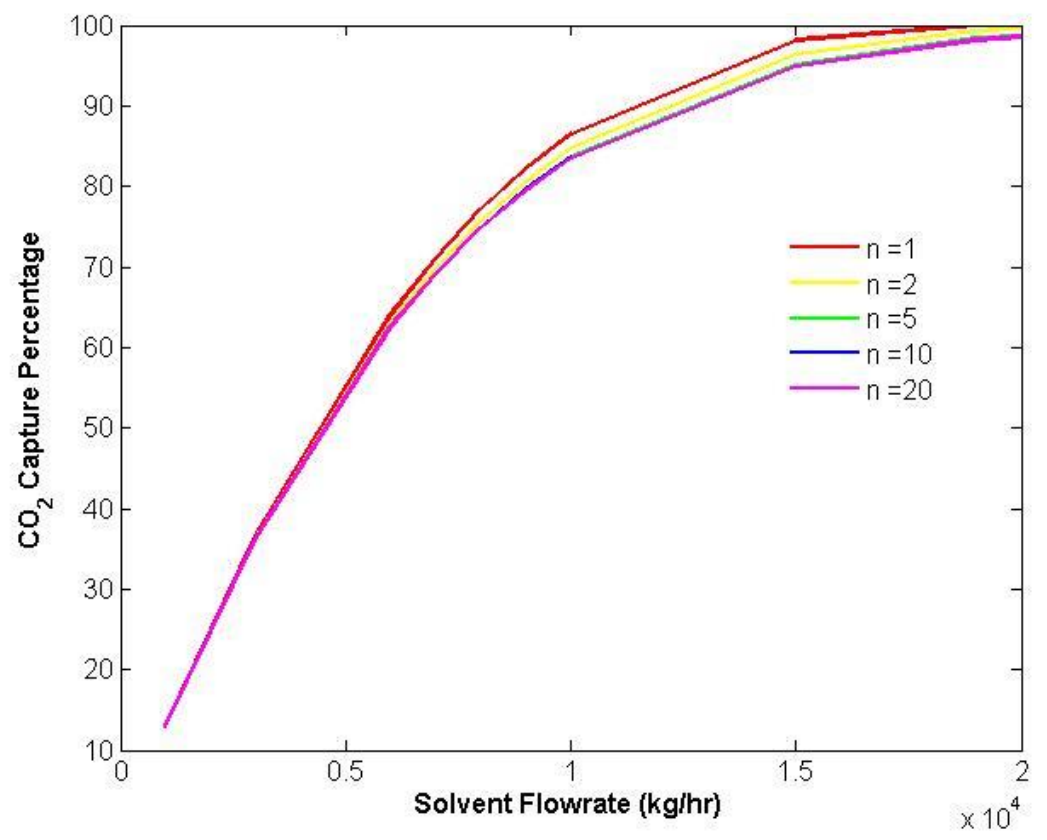

Figure F.2. Effect of column discretization on absorber $\mathrm{CO}_{2}$ capture percentage predicted in scale-up uncertainty quantification.

For this form of the liquid distribution function, a relatively small number of discretizations is required for the $\mathrm{CO}_{2}$ capture percentage to approach a constant value for the entire range of liquid flowrate. Therefore, a value of $n=10$ is chosen here to avoid increased computational expense associated with a larger number of discretizations. Another sensitivity study is performed to determine the effect of the relative column radius on $\mathrm{CO}_{2}$ capture percentage, and the results are given in Figure F.3.

A

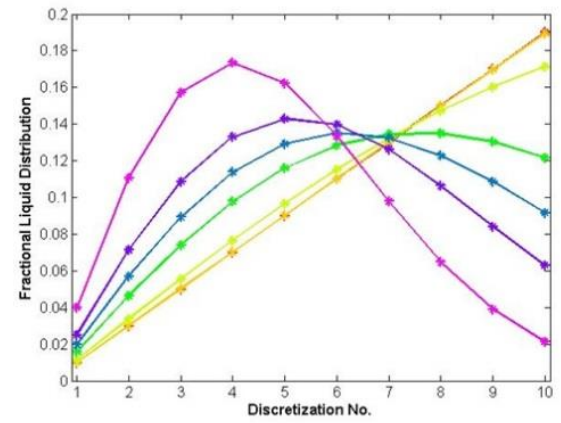

$\mathrm{B}$

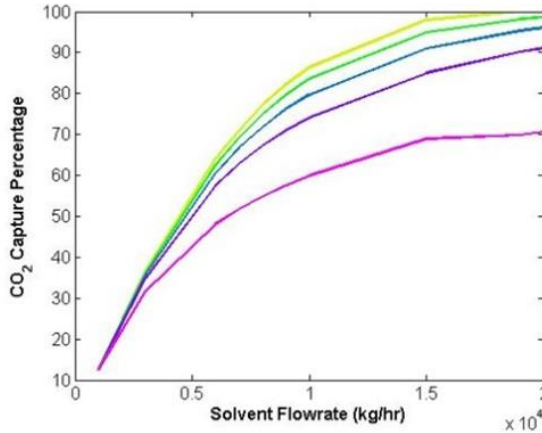

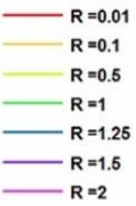

Figure F.3. Effect of relative radius value on performance of absorber column with 10 discretizations. (A) Fraction of overall amount of liquid appropriated to each section. (B) $\mathrm{CO}_{2}$ capture percentage as a function of solvent flowrate. 
As the value of the relative radius approaches zero, the column operation approximates the uniform distribution case and the fraction of liquid contained in each segment is proportional to the area of the segment. With increasing radius, the column operation becomes increasingly inefficient as less liquid is contained in the larger outer segments of the column. This analysis is intended to provide an example of how liquid maldistribution may affect the performance of an absorber column.

For calibrating the parameters of the model for the scale-up UQ work, this methodology will be applied to the absorber column at the Technology Centre Mongstad (TCM) pilot plant, and this model will be developed specifically for FLEXIPAC $2 X$ packing. Although the column directions are referred to as axial and radial in this work, the actual absorber column has a rectangular cross section. Some data have been obtained from a recent MEA campaign at this pilot plant, and more are planned to be obtained in a future campaign and used for a rigorous validation of this methodology. In another CCSI research project, MEA solvent model developed in this work has been scaled-up to the TCM pilot plant specification, and has been shown to predict absorber and stripper column performance that matches well with the data from this pilot plant campaign. An example case from the TCM data set is used for this analysis, and essential variables are given in Table F.2.

Table F.2. Process and configuration variables for TCM absorber column sample case

\begin{tabular}{cc}
\hline Variable & Value \\
\hline Inlet Lean Solvent Flow (kg/hr) & 114801 \\
Column L:G Mass Ratio & 1.987 \\
Inlet Lean Solvent Loading & 0.2013 \\
Flue Gas $\mathrm{CO}_{2}$ Weight Percentage & 12.8 \\
Absorber Packed Height (m) & 18 \\
Percentage $\mathrm{CO}_{2}$ Capture (Data) & 86.14 \\
Percentage $\mathrm{CO}_{2}$ Capture (Model) & 96.06 \\
\hline
\end{tabular}

Note that the model overpredicts the data value of the percentage $\mathrm{CO}_{2}$ capture, and it is possible that this is in part due to the plug flow Aspen Plus ${ }^{\circledR}$ model not capturing the column inefficiency that results from non-uniform liquid distribution. The comparison of the liquid temperature profile to the model values is given in Figure F.4 for this case. Note that this column includes various temperature sensors across the column cross section, labeled A-D. 


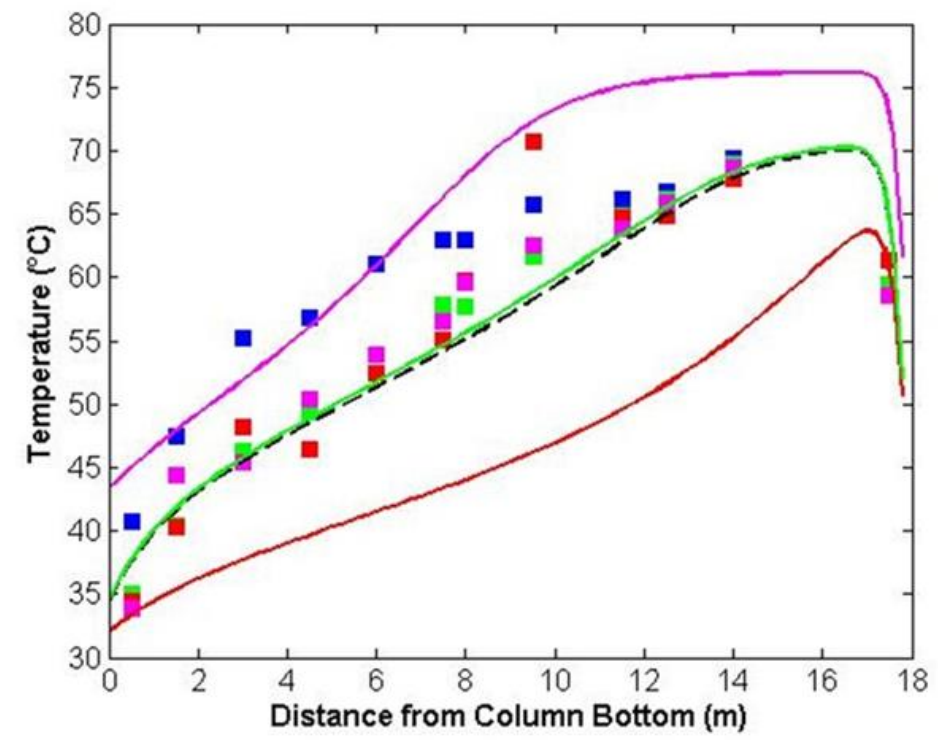

$\underline{\text { Data }}$

- Sensor A

- Sensor B

- Sensor C

- Sensor D

\section{Model Predictions}

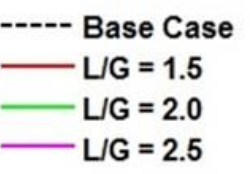

Figure F.4 Temperature sensor measurements for TCM absorber case given in Table F.2. Model predictions are given for base case (described in Table F.2) and with respect to a range of $L: G$ ratio (with constant gas flowrate).

Clearly, the data bed temperature is not uniform in the radial direction, and variation in the local $\mathrm{L}: \mathrm{G}$ ratio may account for some of the discrepancy. This methodology assumes that the capture percentage depends only on the relative ratio of liquid to gas for a section, and not the actual flowrates. This assumption is tested with a sensitivity study in which the liquid and gas flowrates are varied at a constant $\mathrm{L}: \mathrm{G}$ ratio, and the $\mathrm{CO}_{2}$ capture percentage and temperature profiles in the absorber are observed. All other simulation variables used in this study are taken from Table F.2. The results of this sensitivity study are given in Figure F.5, in which $\mathrm{CO}_{2}$ capture percentage is shown as a function of flue gas flowrate at fixed L:G ratio and Figure F.6, in which the temperature profiles are shown for the same comparison. 


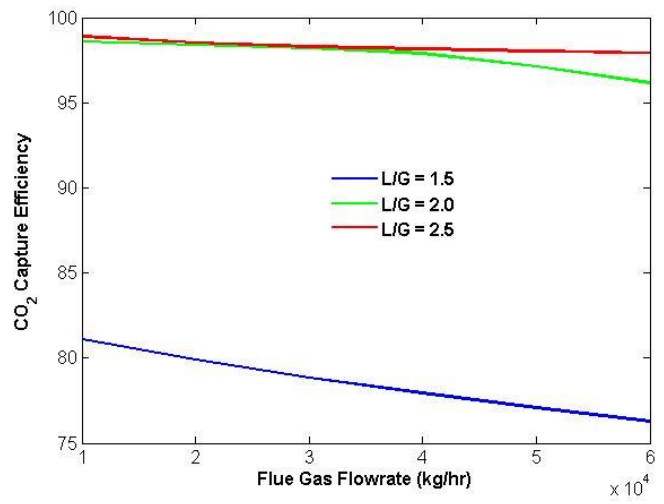

Figure F.5. Effect of flue gas flowrate on $\mathrm{CO}_{2}$ capture percentage at various $\mathrm{L}: \mathrm{G}$ ratios.
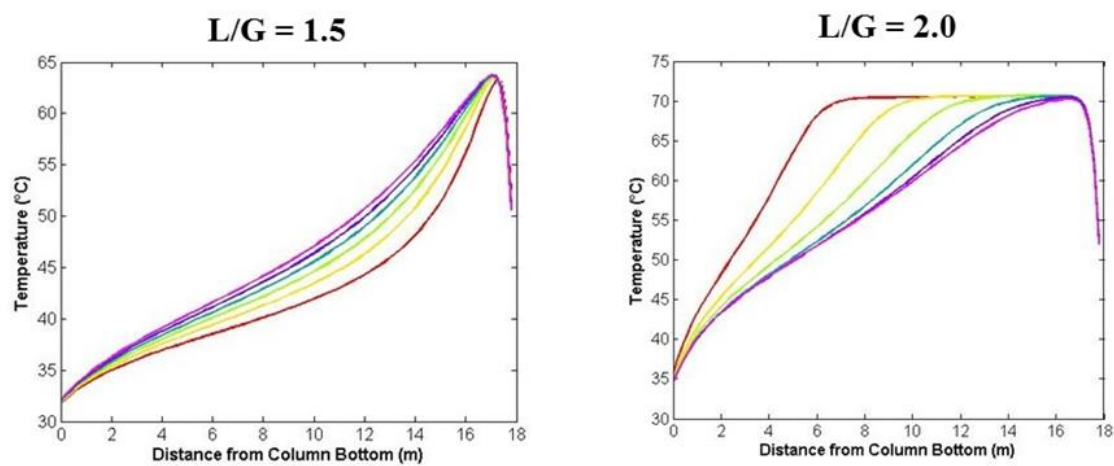

$\mathbf{L} / \mathbf{G}=\mathbf{2 . 5}$

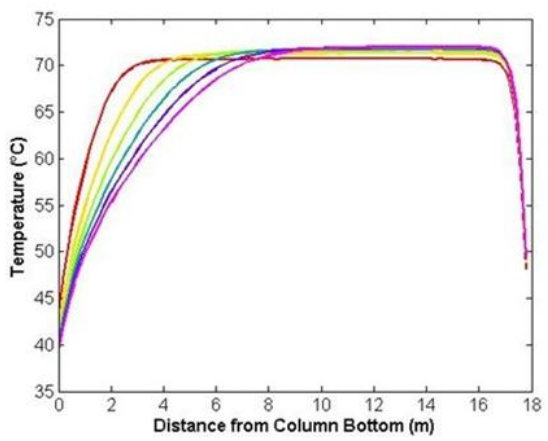

Gas Flowrate $(\mathrm{kg} / \mathrm{hr})$

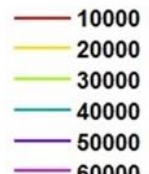

60000

Figure F.6. Effect of flue gas flowrate on absorber temperature profiles at various L:G ratios

As shown in Figure F.5 the $\mathrm{CO}_{2}$ capture percentage varies only slightly over the range 10,000$60,000 \mathrm{~kg} / \mathrm{hr}$ if the $\mathrm{L}: \mathrm{G}$ ratio is constant, although the extent of decrease in $\mathrm{CO}_{2}$ capture percentage increases as the L:G ratio decreases. Some variation in the temperature profiles is also shown in Figure F.6, indicating that the assumption made in Eq. F.4 could contribute some error if used to match temperature profile data. 
Future work will be performed to implement the methodology described here into an Aspen Plus platform so that the form of the liquid distribution and its parameters may be characterized rigorously. Future plant data from TCM will likely be employed as a part of this project. 


\section{References}

Akaike, H., 1974. A new look at the statistical model identification. IEEE Trans. Autom. Control. 19 (6), 716-723.

Amundsen, T.G., Lars, E.Ø., Eimer, D.A., 2009. Density and viscosity of monoethanolamine + water + carbon dioxide from (25 to 80) ${ }^{\circ}$. J. Chem. Eng. Data. 54 (11), 3096-3100.

Aronu, U.E., Gondal, S., Hessen, E.T., Haug-Warberg, T., Hartono, A., Hoff, K.A., Svendsen, H.F., 2011. Solubility of $\mathrm{CO}_{2}$ in $15,30,45$, and 60 mass $\%$ MEA from 40 to $120^{\circ} \mathrm{C}$ and model representation using the extended UNIQUAC framework. Chem. Eng. Sci. 66 (24), 6393-6406.

Artanto, Y., Jansen, J., Pearson, P., Do, T., Cottrell, A., Meuleman, E., Feron, P., 2012. Performance of MEA and amine-based blends in the CSIRO Pilot Plant at Loy Yang Power in Australia. Fuel. 101, 264-275.

Aspen Plus documentation, 2013a. Rate-based Model of the $\mathrm{CO}_{2}$ Capture Process by MEA Using Aspen Plus, V8.4. Aspen Technology, Inc., Cambridge, MA.

Aspen Plus documentation, 2013b. Physical Property System: Physical Property Methods, V8.4. Aspen Technology, Inc., Cambridge, MA.

Aspen Plus documentation, 2013c. Physical Property System: Physical Property Models, V8.4. Aspen Technology, Inc., Cambridge, MA.

Aspen Plus documentation, 2013d. User Models, V8.4. Aspen Technology, Inc., Cambridge, MA.

Asprion, N., 2005. Surface tension models for aqueous amine blends. Ind. Eng. Chem. Res. 44 (18), 7270-7278.

Balakrishnan, S., Roy, A., Ierapetritou, M.G., Flach, G.P., Georgopoulos, P.G., 2003. Uncertainty reduction and characterization for complex environmental fate and transport models: an empirical Bayesian framework incorporating the stochastic response surface method. Water Resour. Res. 39 (12), article 1350.

Beers, K.J., 2007. Probability theory and stochastic simulation, in: Numerical Methods for Chemical Engineering: Applications in MATLAB ${ }^{\circledR}$. Cambridge University Press, New York, pp. $372-435$.

Bland, J.M., Altman, D.G., 1986. Statistical methods for assessing agreement between two methods of clinical measurement. 327, 307-310. 
Bontinck, Z., De Gersem, H., Schöps, S., 2016. Response surface models for the uncertainty quantification of eccentric permanent magnet synchronous machines. IEEE T. Magn. 52 (3), article 72030404.

Boot-Handford, T.G., Abanades, J.C., Anthony, E.J., Blunt, M.J., Brandani, S., MacDowell, N., Fernández, J.R., Ferrari, M.C., Gross, R., Hallett, J.P., Haszeldine, R.S., Heptonstall, P., Lyngfelt, A., Makuch, Z., Mangano, E., Porter T.R.J., Pourkashanian, M., Rochelle, G.T., Shah, N., Yao, J.G., Fennell, P.S., 2014. Carbon capture and storage update. Energy Environ. Sci. 7 (1), 130-189.

Cai, Z., Xie, R., Wu, Z., 1996. Binary isobaric vapor-liquid equilibria of ethanolamines + water. J. Chem. Eng. Data. 41 (5), 1101-1103.

Chaloner, K.C., Verdinelli, I., 1995. Bayesian experimental design: a review. Stat. Sci. 10 (3), 273-304.

Chen, C.C., Britt, H.I., Boston, J.F., Evans, L.B., 1982. Local composition model for excess Gibbs energy of electrolyte systems part 1: single solvent, single completely dissociated electrolyte systems. AIChE J. 28 (4), 588-596.

Chinen, A.S., Morgan, J.C., Omell, B., Bhattacharyya, D., Tong, C., Miller, D.C., 2017. Development of a gold-standard model for solvent-based $\mathrm{CO}_{2}$ capture. Part 1: Hydraulic and mass transfer models and their uncertainty quantification. (in preparation)

deMontigny, D., Tontiwachwuthikul, P., Chakma, A., 2001. Parametric studies of carbon dioxide absorption into highly concentrated monoethanolamine solutions. Can. J. Chem. Eng. 79 (1), 137-142.

Der Kiureghian, A., Ditlevsen, O., 2007. Aleatory or epistemic? Does it matter? Paper presented at Special Workshop on Risk Acceptance and Risk Communication, Stanford University.

Dinca, C., Badea, A., 2013. The parameters optimization of a CFBC pilot plant experimental study of a post-combustion $\mathrm{CO}_{2}$ capture by reactive absorption with MEA. Int. J. Greenh. Gas Con. 12, 269-279.

Dugas, R.E., 2009. Carbon Dioxide Absorption, Desorption, and Diffusion in Aqueous Piperazine and Monoethanolamine. Ph.D. Thesis. The University of Texas at Austin, 2009.

Dugas, R., Alix, P., Lemaire, E., Broutin, P., Rochelle, G., 2009. Absorber model for $\mathrm{CO}_{2}$ capture by monoethanolamine - application to CASTOR pilot results. Energy Procedia. 1, 103107. 
Faramarzi, L., Kontogerogis, G.M., Michelsen, M.L., Thomsen, K., Stenby, E.H., 2010. Absorber model for $\mathrm{CO}_{2}$ capture by monoethanolamine. Ind. Eng. Chem. Res. 49 (8), 37513759 .

Friedman, J.H., 1991. Multivariate adaptive regression splines. Ann. Stat. 19 (1), 1-141.

Gel, A., Li, T., Goplan, B., Shahnam, M., Syamlal, M., 2013. Validation and uncertainty quantification of a multiphase computational fluid dynamics model. Ind. Eng. Chem. Res. 52(33), 11424-11435.

Gel, A., Chaudhari, K., Turton, R., Nicoletti, P., 2014. Application of uncertainty quantification methods for coal devolatilization kinetics in gasifier modeling. Powder Technol. 265, 66-75.

Gibbins, J., Chalmers, H., 2008. Carbon capture and storage. Energy Policy. 36 (12), 4317-4322.

Giunta, A.A., McFarland, J.M., Swiler, L.P., Eldred, M.S., 2006. The promise and peril of uncertainty quantification using response surface approximations. Struct. Infrastruct. E. 2 (3-4), 175-189.

Gjernes, E., Pedersen, S., Cents, T., Watson, G., Fostås, B.F., Shah, M.I., Lombardo, G., Desvignes, C., Flø, N.E., Morken, A.K., de Cazenove, T., Faramarzi, L., Hamborg, E.S., 2016. Results from $30 \mathrm{wt} \%$ MEA performance testing at the $\mathrm{CO}_{2}$ Technology Centre Mongstad. Paper presented at the $13^{\text {th }}$ International Conference on Greenhouse Gas Control Technologies (GHGT13), Lausanne, Switzerland.

Han, B., Zhou, C., Wu, J., Tempel, D.J., Cheng, H., 2011. Understanding $\mathrm{CO}_{2}$ capture mechanisms in aqueous monoethanolamine via first principles simulations. J. Phys. Chem. Lett. $2(6), 522-526$.

Han, C., Graves, K., Neathery, J., Kunlei, L., 2011. Simulation of the energy consumption of $\mathrm{CO}_{2}$ capture by aqueous monoethanolamine in pilot plant. Energy Environ. Res. 1 (1), 67-80.

Han, J., Jin, J., Eimer, D.A., Melaaen, M.C., 2012. Density of water (1) + monoethanolamine (2) $+\mathrm{CO}_{2}$ (3) from (298.15 to 413.15) $\mathrm{K}$ and surface tension of water (1) + monoethanolamine (2) from (303.15 to 333.15) K. J. Chem. Eng. Data. 57 (4), 1095-1103.

Haszeldine, R.S., 2009. Carbon capture and storage: how green can black be? Science. 325 (5948), 1647-1652.

Hilliard, M.D., 2008. A Predictive Thermodynamic Model for an Aqueous Blend of Potassium Carbonate, Piperazine, and Monoethanolamine for Carbon Dioxide Capture from Flue Gas. Ph.D. Thesis. The University of Texas at Austin. 
Hogg, R.V., Craig, A.T., 1978. The multivariate normal distribution, in: Introduction to Mathematical Statistics, fourth ed. Macmillan Publishing Co., Inc., New York, pp. 405-410.

Idem, R., Wilson, M., Tontiwachwuthikul, P., Chakma, A., Veawab, A., Aroonwilas, A., Gelowitz, D., 2006. Pilot plant studies of the $\mathrm{CO}_{2}$ capture performance of aqueous MEA and mixed MEA/MDEA solvents at the University of Regina $\mathrm{CO}_{2}$ Capture Technology Development Plant and the Boundary Dam $\mathrm{CO}_{2}$ Capture Demonstration Plant. Ind. Eng. Chem. Res. 45 (8), 2414-2420.

Jayarathna, S.A., Weerasooriya, A., Dayarathna, S., Eimer, D.A., Melaaen, M.C., 2013. Densities and surface tensions of $\mathrm{CO}_{2}$ loaded aqeous monoethanolamine solutions with $\mathrm{r}=(0.2$ to $0.7)$ at $\mathrm{T}=(303.15$ to 333.15$)$ K. J. Chem. Eng. Data. 58 (4), 986-992.

Jou, F.Y., Mather, A.E., Otto, F.D., 1995. The solubility of $\mathrm{CO}_{2}$ in a 30 mass percent monoethanolamine solution. Can. J Chem. Eng. 73 (1), 140-147.

Kaewsichan, L., Al-Bofersen, O., Yesavage, V.F., Selim, M.S., 2001. Predictions of the solubility of acid gases in monoethanolamine (MEA) and methyldiethanolamine (MDEA) solutions using the electrolyte-UNIQUAC model. Fluid Phase Equilibr. 183-184, 159-171.

Kim, I., Svendsen, H.F., 2007. Heat of absorption of carbon dioxide $\left(\mathrm{CO}_{2}\right)$ in monoethanolamine (MEA) and 2-(Aminoethyl) ethanolamine (AEEA) solutions. Ind. Eng. Chem. Res. 46 (17), 5803-5809.

Kim, I., Hoff, K.A., Mejdell, T., 2014. Heat of absorption of $\mathrm{CO}_{2}$ with aqueous solutions of MEA: new experimental data. Energy Procedia. 63, 1446-1455.

Kim, S., Kang, J., Kroenlein, K., Magee, J.W., Diky, V., Frenkel, M., 2013. Online resources in chemical engineering education: impact of the uncertainty concept from thermophysical properties. Chem. Eng. Ed. 47(1), 48-57.

Knudsen, J.N., Jensen, J.N., Vilhelmsen, P., Biede, O., 2009. Experience with $\mathrm{CO}_{2}$ capture from coal flue gas in pilot-scale: testing of different amine solvents. Energy Procedia. 1, 783-790.

Kohl, A.L., Nielsen, R., 1997. Mechanical design and operation of alkanolamine plants, in: Gas Purification, fifth ed. Gulf Publishing Company, Houston, TX, pp. 187-277.

Kwak, N., Lee, J.H., Lee, I.Y., Jang, K.R., Shim, J., 2012. A study of the $\mathrm{CO}_{2}$ capture pilot plant by amine absorption. Energy. 47, 41-46.

Lane, W.A., Storlie, C.B., Montgomery, C.J., Ryan, E.M., 2014. Numerical modeling and uncertainty quantification of a bubbling fluidized bed with immersed horizontal tubes. Powder Technol. 253, 733-743. 
Lee, J.I., Otto, F.D., Mather, A.E., 1976. Equilibrium between carbon dioxide and aqueous monoethanolamine solutions. J. Appl. Chem. Biotechn. 26 (1), 541-549.

Liley, P.E., Thomson, G.H., Friend, D.G., Daubert, T.E., Buck, E., 1997. Physical and chemical data, in: Perry's Chemical Engineers' Handbook, seventh ed. McGraw-Hill, New York.

Luo, X., Knudsen, J.N., de Montigny, D., Sanpasertparnich, T., Idem, R., Gelowitz, D., 2009. Comparison and validation of simulation codes against sixteen sets of data from four different pilot plants. Energy Procedia. 1, 1249-1256.

Ma, Z., Zabaras, N., 2009. An efficient Bayesian inference approach to inverse problems based on adaptive sparse grid collection method. Inverse Probl. 25(3), article 035013.

Macchietto, S., Maduabeuke, G., Szczepanski, R., 1986. Exact determination of process sensitivity to physical properties. Fluid Phase Equilib. 29, 59-67.

Ma'mun, S., Nilsen, R., Svendsen, H.F., 2005. Solubility of carbon dioxide in 30 mass\% monoethanolamine and 50 mass\% methyldiethanolamine solutions. J. Chem. Eng. Data. 50 (2), 630-634.

Mangalapally, H.P., Hasse, H., 2011a. Pilot plant study of post-combustion carbon dioxide capture by reactive absorption: methodology, comparison of different structured packings, and comprehensive results for monoethanolamine. Chem. Eng. Res. Des. 89 (8), 1216-1228.

Mangalapally, H.P., Hasse, H., 2011b. Pilot plant study of two new solvents for post combustion carbon dioxide capture by reactive absorption and comparison to monoethanolamine. Chem. Eng. Sci. 66 (22), 5512-5522.

Mangalapally, H.P., Hasse, H., 2011c. Pilot plant experiments for post combustion carbon dioxide capture by reactive absorption with novel solvents. Energy Procedia. 4, 1-8.

Mangalapally, H.P., Notz, R., Hoch, S., Asprion, N., Sieder, G., Garcia, H., Hasse, H., 2009. Pilot plant experimental studies of post combustion $\mathrm{CO}_{2}$ capture by reactive absorption with MEA and new solvents. Energy Procedia. 1, 963-970.

Mangalapally, H.P., Notz, R., Asprion, N., Sieder, G., Garcia, H., Hasse, H., 2012. Pilot plant study of four new solvents for post combustion carbon dioxide capture by reactive absorption and comparison to MEA. Int. J. Greenh. Gas. Con. 8, 205-216.

Mathias, P.M., 2014. Sensitivity of process design to phase equilibrium - a new perturbation method based upon the Margules equation. J. Chem. Eng. Data. 59 (4), 1006-1015. 
Mathias, P.M., Gilmartin, J.P., 2014. Quantitative evaluation of the effect of uncertainty in property models on the simulated performance of solvent-based $\mathrm{CO}_{2}$ capture. Energy Procedia. 63, 1171-1185.

Mebane, D.S., Bhat, K.S., Kress, J.D., Fauth, D.J., Gray, M.L., Lee, A., Miller, D.C., 2013. Bayesian calibration of thermodynamic models for the uptake of $\mathrm{CO}_{2}$ in supported amine sorbents using ab initio priors. Phys. Chem. Chem. Phys. 15, 4355-4366.

Miller, D.C., Syamlal M., Mebane, D.S., Storlie C., Bhattacharyya D., Sahinidis N.V., Agarwal D., Tong C., Zitney S.E., Sarkar A., Sun X., Sundaresan S., Ryan E., Engel D., Dale C., 2014. Carbon capture simulation initiative: a case study in multiscale modeling and new challenges. Annu. Rev. Chem. Biomol. Eng. 5, 301-323.

Mišković, L., Hatzimanikatis, V., 2011. Modeling of uncertainties in biochemical reactions. Biotechnol. Bioeng. 108 (2), 413-423.

Mohamed Ali, A., Jansens, P.J., Olujić, Z., 2003. Experimental characterization and computational fluid dynamics simulation of gas distribution performance of liquid (re)distributors and collectors in packed columns. Trans IChemE. 81, 108-115.

Mores, P., Scenna, N., Mussati, S., 2011. Post-combustion $\mathrm{CO}_{2}$ capture process: equilibrium stage mathematical model of the chemical absorption of $\mathrm{CO}_{2}$ into monoethanolamine (MEA) aqueous solution. Chem. Eng. Res. Des. 89 (9), 1587-1599.

Morgan, J.C., Bhattacharyya, D., Tong, C., Miller, D.C., 2015. Uncertainty quantification of property models: methodology and its application to $\mathrm{CO}_{2}$-loaded aqueous MEA solutions. AIChE J. 61 (6), 1822-1839.

Morgan, J.C., Chinen, A.S., Omell, B., Bhattacharyya, D., Tong, C., Miller, D.C., 2017a. Thermodynamic modeling and uncertainty quantification of $\mathrm{CO}_{2}$-loaded aqueous MEA solutions. Chem. Eng. Sci. 168, 309-324.

Morgan, J.C., Chinen, A.S., Omell, B., Bhattacharyya, D., Tong, C., Miller, D.C., Wheeldon, J., Buschle, B., Lucquiaud, M., 2017b. Development of a gold-standard model for solvent-based $\mathrm{CO}_{2}$ capture. Part 2: Development of steady-state process model for MEA system and validation with pilot plant data. (in preparation)

Moser, P., Schmidt, S., Sieder, G., Garcia, H., Stoffregen, T., 2011a. Performance of MEA in a long-term test at the post-combustion capture pilot plant in Niederaussem. Int J. Greenh. Gas Con. 5, 620-627. 
Moser, P., Schmidt, S., Stahl, K., 2011b. Investigation of trace elements in the inlet and outlet streams of a MEA-based post-combustion capture process results from the test programme at the Niederaussem Pilot Plant. Energy Procedia. 4, 473-479.

Moser, P., Schmidt, S., Sieder, G., Garcia, H., Stoffregen, T., Stamatov, V., 2011c. The postcombustion capture pilot plant Niederaussem - results of the first half of the testing programme. Energy Procedia. 4, 1310-1316.

Nawrocki, P.A., Xu, Z.P., Chuang, K.T., 1991. Mass transfer in structured corrugated packing. Can. J. Chem. Eng. 69 (6), 1336-1343.

Notz, R., Mangalapally, H.P., Hasse, H. Post combustion $\mathrm{CO}_{2}$ capture by reactive absorption: pilot plant description and results of systematic studies with MEA. Int. J. Greenh. Gas Con. 12, 84-112.

Nuchitprasittichai, A., Cremaschi, S., 2013. Sensitivity of amine-based $\mathrm{CO}_{2}$ capture cost: the influences of $\mathrm{CO}_{2}$ concentration in flue gas and utility cost fluctuations. Int. J. Greenh. Gas Con. $13,34-43$.

Papadimitriou, C., 2014. Bayesian uncertainty quantification and propagation in structural dynamics simulations. Paper presented at International Conference on Structural Dynamics, Porto, Portugal.

Plaza, J.M., 2012. Modeling of Carbon Dioxide Absorption Using Aqueous Monoethanolamine, Piperazine, and Promoted Potassium Carbonate. Ph.D. Thesis. The University of Texas at Austin.

Press, W.H., Teukolsky, S.A., Vetterling, W.T., Flannery, B.P., 1992. Modeling of data, in: Numerical Recipes in C: the Art of Scientific Computing, second ed. Cambridge University Press, New York, pp. 656-706.

Rabensteiner, M., Kinger, G., Koller, M., Gronald, G., Hochenauer, C., 2014. Pilot plant study of ethylenediamine as a solvent for post combustion carbon dioxide carbon capture and comparison to monoethanolamine. Int. J. Greenh. Gas Con. 27, 1-14.

Rubin, E.S., Davison, J.E., Herzog, H.J., 2015. The cost of $\mathrm{CO}_{2}$ capture and storage. Int. J. Greenh. Gas. Con. 40, 378-400.

Saimpert, M., Puxty, G., Qureshi, S., Wardhaugh, L., Cousins, A., 2013. A new rate based absorber and desorber modelling tool. Chem. Eng. Sci. 96, 10-25. 
Sarkar, S., Kosson, D.S., Mahadevan, S., Meeussen, J.C., van der Sloot, H., Arnold, J.R., Brown, K.G., 2012. Bayesian calibration of thermodynamic parameters for geochemical speciation modeling of cementitious materials. Cem. Concr. Res. 42 (7), 889-902.

Simon, L.L., Elias, Y., Puxty, G., Artanto, Y., Hungerbuhler, K., 2011. Rate based modeling and validation of a carbon-dioxide pilot plant absorption column operating on monoethanolamine. Chem. Eng. Res. Des. 89 (9), 1684-1692.

Sobol, I.M., 1993. Sensitivity estimates for nonlinear mathematical models. Math Model. Comput. Exp. 1, 407-414..

Sønderby, T.L., Carlsen, K.B., Fosbøl, P.L., Kiørboe, L.G., von Solms, N., 2013. A new pilot absorber for $\mathrm{CO}_{2}$ capture from flue gases: measuring and modelling capture with MEA solution. Int. J. Greenh. Gas Con. 12, 181-192.

Sudret, B., 2012. Meta-models for structural reliability and uncertainty quantification. Paper presented at Fifth Asian-Pacific Symposium on Structural Reliability and its Applications, Singapore.

Tobiesen, F.A., Svendsen, H.F., Juliussen, O., 2007. Experimental validation of a rigorous absorber model for $\mathrm{CO}_{2}$ postcombustion capture. AIChE J. 53 (4), 846-865.

Tobiesen, F.A., Juliussen, O., Svendsen, H.F., 2008. Experimental validation of a rigorous desorber model for $\mathrm{CO}_{2}$ post-combustion capture. Chem. Eng. Sci. 63 (10), 2641-2656.

Tochigi, K., Akimoto, K., Ochi, K., Liu, F., Kawase, Y., 1999. Isothermal vapor-liquid equilibria for water + 2-aminoethanol + dimethyl sulfoxide and its constituent three binary systems. J. Chem. Eng. Data. 44 (3), 588-590.

Tönnies, I., Mangalapally, H.P., Hasse, H., 2011. Sensitivity study for the rate-based simulation of the reactive absorption of $\mathrm{CO}_{2}$. Energy Procedia. 4, 533-540.

Turton, R., Bailie, R.C., Whiting, W.B., Shaeiwitz, J.A., Bhattacharyya, D., 2012. Synthesis of a process using a simulator and simulator troubleshooting, in: Analysis, Synthesis, and Design of Chemical Processes, fourth ed. Prentice Hall, Upper Saddle River, NJ, pp. 385-450.

Van Wagener, D.H., 2011. Stripper Modeling for $\mathrm{CO}_{2}$ Removal Using Monoethanolamine and Piperazine Solvents. Ph.D. Thesis. The University of Texas at Austin. 
Vrachnos, A., Kontogeorgis, G., Voutsas, E., 2006. Thermodynamic modeling of acidic gas solubility in aqueous solutions of MEA, MDEA, and MEA-MDEA blends. Ind. Eng. Chem. Res. 45 (14), 5148-5154.

Weast, R.C., 1984. Handbook of Chemistry and Physics, $65^{\text {th }}$ ed. CRC Press, Boca Raton, FL.

Weaver, B.P., Williams, B.J., Anderson-Cook, C.M., Higdon, D.M., 2016. Computational enhancements to Bayesian design of experiments using Gaussian processes. Bayesian Anal. 11 (1), 191-213.

Weber, C.L., Vanbriesen, J.M., Small, M.S., 2006. A stochastic regression approach to analyzing thermodynamic uncertainty in chemical speciation modeling. Environ. Sci. Technol. 40 (12), 3872-3878.

Weiland, R.H., Dingman, J.C., Cronin, D.B., 1997. Heat capacity of aqueous monoethanolamine, diethanolamine, n-methyldiethanolamine, and n-methyldiethanolamine-based blends with carbon dioxide. J. Chem. Eng. Data. 42 (5), 1004-1006.

Weiland, R.H., Dingman, J.C., Cronin, B., Browning, G.J., 1998. Density and viscosity of some partially carbonated aqueous alkanolamine solutions and their blends. J. Chem. Eng. Data. 43 (3), 378-382.

Whiting, W.B., 1996. Effects of uncertainties in thermodynamic data and models on process calculations. J. Chem. Eng. Data. 41 (5), 935-941.

Wilson, M., Tontiwachwuthikul, P., Chakma, A., Idem, R., Veawab, A., Aroonwilas, A., Gelowitz, D., Barrie, J., Mariz, C., 2004. Test results from a $\mathrm{CO}_{2}$ extraction pilot plant at Boundary Dam Coal-Fired Power Station. Energy. 29, 1259-1267.

$\mathrm{Xu}, \mathrm{Q} ., 2$ 2011. Thermodynamics of $\mathrm{CO}_{2}$ loaded Aqueous Amines. Ph.D. Thesis. The University of Texas at Austin.

Zhang, Y., Chen, H., Chen, C., Plaza, J.M., Dugas, R., Rochelle, G.T., 2009. Rate-based process modeling study of $\mathrm{CO}_{2}$ capture with aqueous monoethanolamine solution. Ind. Eng. Chem. Res. 48 (20), 9233-9246.

Zhang, Y., Que, H., Chen, C.C., 2011. Thermodynamic modeling for $\mathrm{CO}_{2}$ absorption in aqueous MEA solution with electrolyte NRTL model. Fluid Phase Equilib. 311, 67-75.

Zhang, Y., Chen, C., 2013. Modeling $\mathrm{CO}_{2}$ absorption and desorption by aqueous monoethanolamine solution with Aspen rate-based model. Energy Procedia. 37, 1584-1596. 